\title{
LEARNING THE NEURON FUNCTIONS WITHIN NEURAL NETWORKS BASED ON GENETIC PROGRAMMING
}

\author{
by \\ Alan J. Barton

\begin{abstract}
A thesis submitted to
the Faculty of Graduate Studies and Research

in partial fulfillment of

the requirements for the degree of

MASTER OF COMPUTER SCIENCE

Ottawa-Carleton Institute for Computer Science

School of Computer Science

at
\end{abstract} \\ CARLETON UNIVERSITY
}

Ottawa, Ontario, Canada

January, 2009

Copyright by Alan J. Barton, 2009 


$\begin{array}{ll}\begin{array}{l}\text { Library and } \\ \text { Archives Canada }\end{array} & \begin{array}{l}\text { Bibliothèque et } \\ \text { Archives Canada }\end{array} \\ \begin{array}{l}\text { Published Heritage } \\ \text { Branch }\end{array} & \begin{array}{l}\text { Direction du } \\ \text { Patrimoine de l'édition }\end{array} \\ \begin{array}{l}\text { 395 Wellington Street } \\ \text { Ottawa ON K1A 0N4 } \\ \text { Canada }\end{array} & \begin{array}{l}\text { 395, rue Wellington } \\ \text { Ottawa ON K1A 0N4 } \\ \text { Canada }\end{array}\end{array}$

Your file Votre référence ISBN: 978-0-494-47527-0 Our file Notre référence ISBN: 978-0-494-47527-0

NOTICE:

The author has granted a nonexclusive license allowing Library and Archives Canada to reproduce, publish, archive, preserve, conserve, communicate to the public by telecommunication or on the Internet, loan, distribute and sell theses worldwide, for commercial or noncommercial purposes, in microform, paper, electronic and/or any other formats.

The author retains copyright ownership and moral rights in this thesis. Neither the thesis nor substantial extracts from it may be printed or otherwise reproduced without the author's permission.
AVIS:

L'auteur a accordé une licence non exclusive permettant à la Bibliothèque et Archives Canada de reproduire, publier, archiver, sauvegarder, conserver, transmettre au public par télécommunication ou par l'Internet, prêter, distribuer et vendre des thèses partout dans le monde, à des fins commerciales ou autres, sur support microforme, papier, électronique et/ou autres formats.

L'auteur conserve la propriété du droit d'auteur et des droits moraux qui protège cette thèse. $\mathrm{Ni}$ la thèse ni des extraits substantiels de celle-ci ne doivent être imprimés ou autrement reproduits sans son autorisation.
In compliance with the Canadian Privacy Act some supporting forms may have been removed from this thesis.

While these forms may be included in the document page count, their removal does not represent any loss of content from the thesis.
Conformément à la loi canadienne sur la protection de la vie privée, quelques formulaires secondaires ont été enlevés de cette thèse.

Bien que ces formulaires aient inclus dans la pagination, il n'y aura aucun contenu manquant.

\section{Canada}


To my parents, twin sister, her husband and two kids, and Grandma. Grandad, I know you were watching over me all these years. With Love... Alan

Consequently I rejoice, having to construct something Upon which to rejoice T.S. Eliot. Ash Wednesday (l.24-25) 


\section{Table of Contents}

List of Tables $\quad$ xi

List of Figures $\quad$ xii

Nomenclature xiv

$\begin{array}{ll}\text { Abstract } & \mathbf{x x} \\ & \mathbf{x}\end{array}$

Acknowledgements $\quad$ xxi

Chapter 1 Introduction 1

1.1 Problem Staternent . . . . . . . . . . . . . . . . 1

1.2 Motivation for Pursuing this Research . . . . . . . . . . . . 2

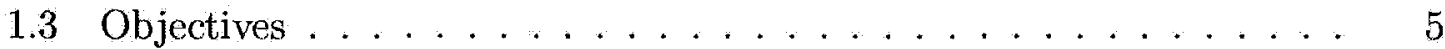

1.4 Research Statement . . . . . . . . . . . . . . . . 6

1.5 Overview of Results . . . . . . . . . . . . . . . 7

1.6 Organization of Thesis ................... 8

1.7 Chapter Summary . . . . . . . . . . . . . . 9

$\begin{array}{lll}\text { Chapter } 2 \text { Background } & 10\end{array}$

2.1 Before Constructing an Algorithm: Initial Considerations . . . . . . 10

2.1.1 The Domain and its Data .............. . . 10

2.1 .2 The Algorithm ..................... 11

2.1.3 The Search Methodology . . . . . . . . . . . . 11

2.1 .4 The Internal Representation . . . . . . . . . . . . 12

2.1.5 The Results (The External Representation) . . . . . . . . 12

2.2 Foundation Algorithms . . . . . . . . . . . . . . . . 12

2.2.1 A General Specification for Evolutionary Computation . . . 12

2.2 .2 A Genetic Algorithm . . . . . . . . . . . . . . 16 
2.2.2.1 Internal Representations . . . . . . . . . . 17

2.2 .2 .2 Operators . . . . . . . . . . . . . 18

2.2.3 A Genetic Programming Algorithm . . . . . . . . . . . 19

2.2.3.1 Internal Representation . . . . . . . . . . 19

2.2 .3 .2 Operators . . . . . . . . . . . . . 20

2.2.3.3 Primary Operator: Crossover (sexual recombination) 20

2.2.3.4 Primary Operator: Reproduction . . . . . . . . 20

2.2.3.5 Secondary Operator: Mutation . . . . . . . . 21

2.2.3.6 Secondary Operator: Permutation (Inversion) . . . 21

2.2.3.7 Secondary Operator: Editing . . . . . . . . . . 21

2.2.3.8 Secondary Operator: Encapsulation . . . . . . . . 22

2.2.3.9 Secondary Operator: Decimation . . . . . . . . 22

2.2.4 A Gene Expression Programming Algorithm . . . . . . . . 22

2.2.4.1 Internal Representation . . . . . . . . . . . 23

2.2 .4 .2 Operators . . . . . . . . . . . . . 27

2.2.5 An Artificial Neural Network (ANN) Algorithm . . . . . . . 29

2.2.5.1 General Neural Network . . . . . . . . . . . . 29

2.2.5.2 Special Case: Feed Forward Neural Network . . . . . 30

2.2.5.3 Type of Neural Network used in this Thesis . . . . 32

2.2.5.4 First Model of a Neuron . . . . . . . . . . . 32

2.2.5.5 Perceptron . . . . . . . . . . . . . 33

2.2.5.6 Training via Classical Backpropagation . . . . . . . 34

2.3 Other Background Information $\ldots \ldots \ldots \ldots \ldots \ldots$

2.3.1 Rough Sets Theory . . . . . . . . . . . . . . . 36

2.3.1.1 Reducts and Minimum Reducts . . . . . . . . . 36

2.3.1.2 Reduct Computation . . . . . . . . . . . . 36

2.3.2 Individual Dichotomization Algorithm . . . . . . . . 37

2.3 .3 Statistics . . . . . . . . . . . . . . . . . 37

2.3.4 Visualization of High Dimensional Spaces . . . . . . . . 38

2.4 Chapter Summary . . . . . . . . . . . . . . . 43 
Chapter 3 Related Work: State of the Art 44

3.1 Classical Neural Networks . . . . . . . . . . . . . . . . . . . . . 44

3.1 .1 Comparisons . . . . . . . . . . . . . . . . 46

3.1.2 Nonlinear discriminant analysis (NDA) $\ldots \ldots \ldots \ldots . \ldots 47$

3.1.3 Some Alternatives to Classical Networks . . . . . . . . . 47

3.2 Evolution of a Neural Network is Necessary . . . . . . . . . . 48

3.3 Training a Network . . . . . . . . . . . . . . . . . . . . . 49

3.3 .1 Undertraining . . . . . . . . . . . . . . . . . . . 49 49

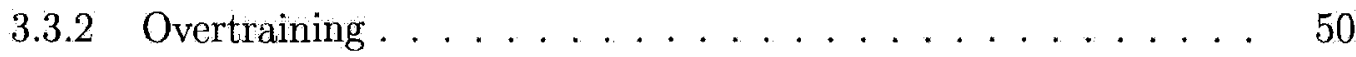

3.3 .3 Input Data is Large . . . . . . . . . . . . . 50

3.3 .4 Input Data is Small . . . . . . . . . . . . . . . . 50

3.3.5 Input Data Contains Missing Points . . . . . . . . . . . . . 51

3.3.6 Presentation Order . . . . . . . . . . . . . . . . . 51

3.3.7 Interpreting A Neural Network (extracting rules) $\ldots \ldots \ldots 51$

3.3.8 Finding More than one Network at a Time . . . . . . . . . 51

3.4 Improving Classical Backpropagation . . . . . . . . . . 52

3.4.1 Improving the Biases and Weights . . . . . . . . . . . 53

3.4 .2 Improving the Architecture $\ldots \ldots \ldots \ldots \ldots 4$

3.4 .3 Improving the Learning Rules $\ldots \ldots \ldots \ldots \ldots \ldots$

3.4.4 Improving the Activation Functions . . . . . . . . . 56

3.4 .5 Neuroevolution . . . . . . . . . . . . . . . 56

3.5 Network Encodings . . . . . . . . . . . . . . . . . . 56

3.5 .1 Direct Encoding . . . . . . . . . . . . . . . . . . 57

3.5 .2 Indirect Encoding . . . . . . . . . . . . . . 58

3.5.3 Common Encoding for Direct and Indirect Encodings . . . . . 58

3.6 Classification . . . . . . . . . . . . . . . . 59

3.6.1 Classification Error Measures . . . . . . . . . . . . . 59

3.6.2 Multiple Class Problem . . . . . . . . . . . . . . . . . 59

3.7 Hybrid Approaches . . . . . . . . . . . . . . . . . . . . 60

3.8 Genetic Programming Variants . . . . . . . . . . . . 60 
3.9 Chapter Summary $\ldots \ldots \ldots \ldots$. . . . . . . . . . . . 61

Chapter 4 NN-GEP Theory $\quad 62$

4.1 A Simple Example . . . . . . . . . . . . . . . . . . . 62

4.2 Representation Scheme for a Problem . . . . . . . . . . . 63

4.2.1 Special Case: FFNN as Automatically Defined Functions . . . 64

4.2.2 Special Case: FFNN for Nonlinear Discriminant Analysis . . . 65

4.2.3 Special Case: FFNN with Multiple Output Layer Neurons . . 65

4.2.4 Special Case: FFNN with Single Output Layer Neuron . . . . 65

4.2 .5 Neural Network Output Vector . . . . . . . . . . 66

4.2.5.1 Output Vector: Number of Output Neurons Equal to Number of Classes . . . . . . . . . . . . 66

4.2.5.2 Output Vector: Single Output Neuron . . . . . . 68

4.2.6 Terminals and Primitive Functions . . . . . . . . . . 70

4.3 Fitness Measure for an Individual . . . . . . . . . . . . . 72

4.3.1 Error Measures $(\mathcal{E})$ used within Fitness . . . . . . . . . 73

4.4 Controlling the Algorithm Search . . . . . . . . . . . . 74

4.5 Terminating the Algorithm: A Heuristic Approach . . . . . . . 76

4.5.1 Example of Termination based on Testing Error $\left(\mathcal{E}_{C}^{T e}\right.$ or $\left.\mathcal{E}_{C_{N}}^{T e}\right) \quad 77$

4.6 Enhancements compared to a Standard GEP Implementation . . . 77

4.7 Chapter Summary . . . . . . . . . . . . . . . . . . 79

Chapter $5 \quad$ NN-GEP Software Engineering and Design $\quad 80$

5.1 Discussion of Design Issues . . . . . . . . . . . . . . 80

5.1.1 The Fitness Function (Processing Algorithm) $\ldots \ldots \ldots 81$

5.1 .2 GEP Class Diagram . . . . . . . . . . . . . . . . 81

5.1 .3 NN-GEP Sequence Diagrams . . . . . . . . . . . 84

5.2 Chapter Summary $\ldots \ldots \ldots \ldots \ldots$

Chapter 6 Experimental Procedures and Testing Methodology 90

6.1 The Data Descriptions . . . . . . . . . . . . . . . . . . 90 
6.1.1 Broad Scale Experiment Settings: Insunza $(c=1) \ldots 90$

6.1.1.1 Insunza Data Preprocessing . . . . . . . . . 93

6.1.2 Broad Scale Experiment Settings: UCI Breast Cancer $(c=2) \quad 94$

6.1.3 Broad Scale Experiment Settings: Werenskiold $(c=5) \quad \ldots \quad 94$

6.1.4 Published: Magnetic Resonance Spectra $(c=4) \ldots 97$

6.1.5 Summary of All Experimental Data Sets . . . . . . . . . . . 100

6.2 The Broad Scale Experimental Methodology . . . . . . . . . . . . 100

6.2.1 Common Broad Scale Experimental Settings for NN-GEP . . 103

6.2.2 Selection Criteria for Broad Scale Experimental Settings . . 103

6.2.3 Computation within One Broad Scale Experiment . . . . . 105

6.2 .4 Computation Time . . . . . . . . . . . . . . . 105

6.2.4.1 Estimated Time Required for MRS . . . . . . . 106

6.2.4.2 Substrate for Aiding Time Reduction . . . . . . . . 106

6.2.5 Policy for Selection of the Best NN-GEP Solutions . . . . . . 107

6.3 Chapter Summary . . . . . . . . . . . . . . . 108

$\begin{array}{lll}\text { Chapter } 7 & \text { Experimental Results } & 109\end{array}$

7.1 Broad Scale Experimental Results . . . . . . . . . . . . . 109

7.1.1 Insunza Broad Scale Results . . . . . . . . . . . . . . . . 111

7.1.1.1 Selected Insunza Results . . . . . . . . . . 115

7.1.1.2 Discussion of Insunza Results . . . . . . . . . 117

7.1.2 Breast Cancer Broad Scale Results . . . . . . . . . . . . . . 121

7.1.2.1 Selected Breast Cancer Results . . . . . . . . . . 121

7.1.2.2 Discussion of Breast Cancer Results . . . . . . . 127

7.1.3 Werenskiold Broad Scale Results . . . . . . . . . . . 128

7.1.3.1 Selected Werenskiold Results for ${ }_{f_{1}} \aleph_{>1}^{\ell_{\text {out }}} \ldots \ldots . . . . .128$

7.1.3.2 Discussion of Werenskiold Results . . . . . . . . . . 132

7.2 Discussion of Parameter Space . . . . . . . . . . . . . . . . 134

7.2 .1 Insunza Parameter Space . . . . . . . . . . . . . . . . . . . 134

7.2 .2 Breast Cancer Parameter Space . . . . . . . . . . . . 137 
7.2.3 Werenskiold Parameter Space . . . . . . . . . . . . . 139

7.3 A Brief Empirical Comparison to Other Techniques . . . . . . . . . 141

7.3.1 NN-GEP vs. a Classical Neural Network . . . . . . . . . . . 141

7.3.2 NN-GEP vs. k-nearest neighbours . . . . . . . . . . . . 142

7.3.3 NN-GEP vs. an Inductive Learner (e.g. decision tree) . . . . 143

7.3.4 NN-GEP vs. GEP with Multiple Chromosomes . . . . . . . 143

7.3.5 NN-GEP vs. Rough Sets . . . . . . . . . . . . . . . 144

7.3.6 NN-GEP vs. Individual Dichotomization . . . . . . . . . 144

7.3.7 NN-GEP vs. a Collection of Neural Networks . . . . . . . 144

7.4 Published Magnetic Resonance Spectra $(c=4)$ Results . . . . . . 145

7.4.1 Published Rough sets Results . . . . . . . . . . . . . . 145

7.4 .2 Published NN-GEP Results . . . . . . . . . . . . 146

7.4.3 Published Individual Dichotomization (Baseline) Results . . . 148

7.5 Chapter Summary . . . . . . . . . . . . . . . . . 150

Chapter $8 \quad$ Summary of work, Conclusions and Future Work 151

8.1 Summary of Thesis Results . . . . . . . . . . . . . . . . 151

8.2 Utility of the Approach . . . . . . . . . . . . . . . . 153

8.3 Thesis Contributions . . . . . . . . . . . . . . . . . 154

8.4 Conclusions Drawn from Results . . . . . . . . . . . . . 154

8.5 Potential Future Directions . . . . . . . . . . . . . . . 155

$\begin{array}{ll}\text { References } & 157\end{array}$

$\begin{array}{lll}\text { Appendix A Example NN-GEP Configuration File } & 175\end{array}$

$\begin{array}{lll}\text { Appendix B Available Symbols within NN-GEP } & 181\end{array}$

Appendix C 6 Experimental Databases 186

C.1 MS Access 2003 Problem . . . . . . . . . . . . . . . . . . 186

C.2 Tables in 1 of the 6 Experimental Databases . . . . . . . . . 187

C.3 Queries in 1 of the 6 Experimental Databases . . . . . . . . 187 
C.4 ID to EXP_ID Conversion Methodology . . . . . . . . . . . . 187

C.5 Using VBA within SQL to Count Number of Constants and Variables 189

C.6 Querying 1 of the 6 Databases for Related Experimental Data . . . 190

C.7 Parameter Space Class Construction . . . . . . . . . . 190

$\begin{array}{lll}\text { Appendix D Further Reading } & 192\end{array}$ 


\section{List of Tables}

$2.1 \quad$ Example of a 3 gene GEP Chromosome $\ldots \ldots \ldots$

4.1 Example 2 Class Problem Confusion Matrix. . . . . . . . . 74

4.2 Common 2 Class Problem Error Measures . . . . . . . . . . 75

4.3 Example demonstrating NN-GEP termination issues. . . . . . 77

$4.4 \quad$ Example of the effect of errors on termination criteria . . . . 78

$6.1 \quad$ Insunza Variable Descriptions . . . . . . . . . . . . . . . . . . 92

6.2 Breast Cancer Variable Descriptions . . . . . . . . . . . 94

6.3 Werenskiold Variable Descriptions . . . . . . . . . 97

6.4 MRS Experimental Settings for Published Results . . . . . . 101

6.5 Metadata for investigated data sets . . . . . . . . . . 102

$6.6 \quad$ Broad Scale Experimental Settings . . . . . . . . . . . . 104

$7.1 \quad$ Summary of all Broad Scale Results . . . . . . . . . . 110

$7.2 \quad$ Pairwise Comparison of Insunza Error Distributions . . . . . 112

7.3 Pairwise Comparison of Breast Cancer Error Distributions . . . 122

7.4 Pairwise Comparison of Werenskiold Error Distributions . . . . 129

$7.5 \quad$ Insunza Parameter Space Class Statistics . . . . . . . . . 135

7.6 Breast Cancer Parameter Space Class Statistics . . . . . . . . 137

7.7 Werenskiold Parameter Space Class Statistics . . . . . . . . 140

$7.8 \quad$ knn Confusion Matrix for all Insunza data $(k=3) \ldots \ldots$

$7.9 \quad \mathrm{j} 48$ Confusion Matrix for all Insunza data . . . . . . . . 143

7.10 Summary of Comparison to Other Techniques . . . . . . . . 150

B.1 Available Functions within NN-GEP . . . . . . . . . . . 181 


\section{List of Figures}

$1.1 \quad$ Prevolution/Evolution and examples of cell types $\ldots \ldots 4$

$1.2 \quad$ Hybridization inspired by missing link (Archaeopteryx) $\ldots .6$

$2.1 \quad$ Mutation, Selection, Environment, Inbreeding, and Races . . . 13

$2.2 \quad$ Processes underlying phenotypic changes in organisms . . . . 13

$2.3 \quad$ Example of a GEP Expression Tree . . . . . . . . . . 23

2.4 Example of a GEP Expression Tree for a gene . . . . . . 25

2.5 Example of a GEP Expression Tree for a gene after mutation . 25

$2.6 \quad$ Example of a GEP Expression Tree for 3 genes . . . . . . 26

$2.7 \quad$ Example GEP ET with head, tail and Dc domains . . . . . 27

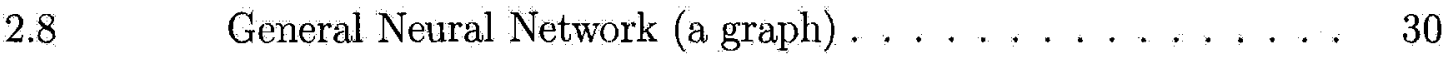

2.9 Special Case: FFNN with Multiple Output Neurons . . . . . 32

$2.10 \quad$ Examples of Linearly Inseparable Problems . . . . . . . . 35

$2.11 \quad[$ Pearson, 1901] Explanation of Regression $\ldots \ldots \ldots \ldots$

$4.1 \quad$ Example of a multicellular system [Ferreira, 2006a] $\ldots \ldots 64$

4.2 Special Case: FFNN Interpreted as learning with ADFs . . . 65

$4.3 \quad$ Special Case: FFNN with one Output Neuron . . . . . . 66

$4.4 \quad$ Neural Network to Individual Mapping . . . . . . . . . 67

$4.5 \quad$ Euclidean Definition of the modulo operation. . . . . . . . 69

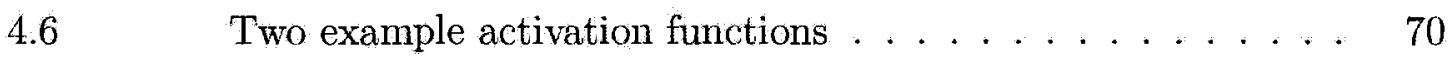

4.7 Deciding between 3 possible single output neuron mappings $\quad$. 71

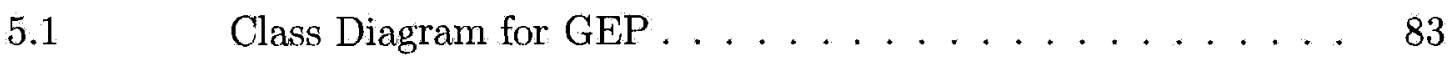

5.2 Sequence Diagram for general startup . . . . . . . . . 84

5.3 Sequence Diagram for initial population construction . . . . 85

5.4 Sequence Diagram for individual evaluation during evolution . 85

$5.5 \quad$ Sequence Diagram listing details of evaluation $\ldots \ldots \ldots 87$

$5.6 \quad$ Sequence Diagram listing details of evaluation (cont.) . . . 88 
$6.1 \quad$ Location of Known Insunza Cave $\ldots \ldots \ldots \ldots$

6.2 Insunza Attribute Distributions $\ldots \ldots \ldots \ldots$. . . . . 92

6.3 Insunza Radioactivity and Local Topography . . . . . . . 93

6.4 Breast Cancer Attribute Distributions ... . . . . . . 95

6.5 Geographic Location of the Werenskiold Glacier . . . . . . 96

$6.6 \quad$ Longitudinal section of the Werenskiold Glacier . . . . . . 97

6.7 Werenskiold Attribute Distributions . . . . . . . . 98

$6.8 \quad$ All (285) Long Echo Time (TE $\geq 130 \mathrm{~ms})$ MR Spectra $\ldots .99$

$7.1 \quad$ Error Distributions $\left(\mathcal{E}_{C}\right)$ for Insunza data $\ldots \ldots \ldots \ldots$

$7.2 \quad$ Error Distributions $\left(\mathcal{E}_{C_{N}}\right)$ for Insunza data $\ldots \ldots \ldots \ldots 114$

$7.3 \quad$ Selected Insunza Results $\left(\mathcal{E}_{C}\right) \ldots \ldots \ldots \ldots \ldots$

$7.4 \quad$ Selected Insunza Results $\left(\mathcal{E}_{C_{N}}\right) \ldots \ldots \ldots \ldots \ldots \ldots$

$7.5 \quad$ Error Distributions $\left(\mathcal{E}_{C}\right)$ for Breast Cancer data $\ldots \ldots \ldots 123$

$7.6 \quad$ Error Distributions $\left(\mathcal{E}_{C_{N}}\right)$ for Breast Cancer data $\ldots \ldots .124$

$7.7 \quad$ Error Distributions $\left(\mathcal{E}_{C}\right)$ for Werenskiold data $\ldots \ldots \ldots$

$7.8 \quad$ Error Distributions $\left(\mathcal{E}_{C_{N}}\right)$ for Werenskiold data $\ldots \ldots \ldots 131$

7.9 Insunza 3D Spaces for NN-GEP Controlling Parameters . . . 136

7.10 Breast Cancer 3D Spaces for NN-GEP Controlling Parameters 138

7.11 Werenskiold 3D Spaces for NN-GEP Controlling Parameters . 139

7.12 Results for best 5 classically trained networks on Insunza data 141

7.13 [Brown, 2004]'s work using collections of neural networks . . . 145

7.14 Best 26 mappings from $\mathbb{R}^{200}$ to $\mathbb{R}^{1}$ for published MRS data . . 147

7.152 (out of 200) variables can discriminate 2 MRS classes . . . 149

C.1 Example Tables for the Insunza Experimental Database . . . 187

C.2 Example Queries for the Insunza Experimental Database . . 188

C.3 Example Join for the Insunza Experimental Database . . . . 190 


\section{Nomenclature}

$\mathcal{T}$ A set of entities. e.g. $\{$ a person, a concept, a visualization, ... $\}$

- A particular entity; in this case, it is the original entity. $(\mathfrak{o} \in \mathcal{T})$

$\mathfrak{r}$ A particular entity; in this case, it is the entity that represents $\mathfrak{o} .(\mathfrak{r} \in \mathcal{T})$

$\mathcal{T}_{\mathfrak{o}} \quad$ A set of original entities $\left(\mathcal{T}_{\mathfrak{0}} \subseteq \mathcal{T}\right)$

$\mathcal{T}_{\mathfrak{r}} \quad$ A set of entities that are representations of original entities $\left(\mathcal{T}_{\mathfrak{r}} \subseteq \mathcal{T}\right)$

$\mathcal{R} \quad$ A family of relations

$\mathcal{R}_{i} \quad$ A particular relation mapping from a subset of $\mathcal{T}$ to a subset of $\mathcal{T}$. $\left(\mathcal{R}_{i}: \mathcal{T}_{\mathfrak{o}} \rightarrow \mathcal{T}_{\mathfrak{r}}\right)$

$\mathbb{P} \quad$ A problem (i.e. a question) that is domain dependent or independent

S A set of solutions (i.e. all possible answers to $\mathbb{P}$ )

$\mathcal{S} \quad$ A particular solution $(\mathcal{S} \subseteq \mathbb{S})$

$\mathcal{S}_{\alpha} \quad$ A representable solution $\left(\mathcal{S}_{\alpha} \subseteq \mathcal{S}\right),\left(\mathcal{S}_{\alpha} \cup \mathcal{S}_{\alpha}=\mathcal{S}\right)$, and $\forall s \in \mathcal{S}_{\alpha} \exists r$ such that $\mathcal{R}_{i}(s)=r$

$\mathcal{S}_{\bar{\alpha}} \quad$ A non-representable solution $\left(\mathcal{S}_{\bar{\alpha}} \subseteq \mathcal{S}\right)$

$\mathcal{S}_{\beta} \quad$ A computable solution $\left(\mathcal{S}_{\beta} \subseteq \mathcal{S}_{\alpha}\right)$ and $\mathcal{S}_{\beta} \cap \mathcal{S}_{\bar{\beta}}=\emptyset$

$\mathcal{S}_{\bar{\beta}} \quad$ A non-computable solution $\left(\mathcal{S}_{\bar{\beta}} \subseteq \mathcal{S}_{\alpha}\right)$

$\mathcal{S}_{\gamma} \quad$ A meaningful solution $\left(\mathcal{S}_{\gamma} \subseteq \mathcal{S}\right)$ and $\mathcal{S}_{\gamma} \cap \mathcal{S}_{\bar{\gamma}}=\emptyset$

$\mathcal{S}_{\bar{\gamma}} \quad$ A meaningless solution $\left(\mathcal{S}_{\bar{\gamma}} \subseteq \mathcal{S}\right)$

$\mathcal{S}_{\gamma \cap \beta} \quad$ A meaningful and computable solution $\left(\mathcal{S}_{\gamma \cap \beta} \subseteq \mathcal{S}_{\gamma} \cap \mathcal{S}_{\beta}\right)$ 
$\left.<x_{1}, x_{2}, \cdots, x_{n}\right\rangle_{i}$ A data set contains $n$ attributes associated to any particular input data object $i$. In other words, when row $i$ is under consideration in the input data, the value in column $j$ is denoted $x_{j}$.

c The number of classes present within a particular data set

$c_{i} \quad$ The $i^{\text {th }}$ class

$n_{c_{i}} \quad$ The number of objects $n$ crisply contained within class $c_{i}$ (i.e. class cardinality) $<n_{c_{1}}, n_{c_{2}}, \cdots, n_{c_{c}}>$ The number of objects crisply contained within all classes in a data set containing $c$ classes

$<n_{c_{1}}, n_{c_{2}}, \cdots, n_{c_{c}}>_{\operatorname{Tr}}$ The data set under consideration is the training data $<n_{c_{1}}, n_{c_{2}}, \cdots, n_{c_{c}}>_{\mathrm{Te}}$ The data set under consideration is the testing data

$c_{\exp } \quad$ Expected class: an object is known to (crisply) belong

$c_{o b s}$ Observed class: an object is predicted to (crisply) belong

$\operatorname{card}(\mathrm{Obj})$ A data set contains a total of $\operatorname{card}(\mathrm{Obj})$ objects. Related notions include: row, example, case, input vector.

$\operatorname{card}($ Attr) A data set contains a total of $\operatorname{card}($ Attr) attributes. Related notions include: column, feature.

$((\operatorname{card}(\mathrm{Obj})=i)$ by $(\operatorname{card}(\mathrm{Attr})=j))$ A specific data set contains $i$ objects and $j$ attributes

$(\operatorname{card}($ Exps $)=11,520)$ The cardinality of the set of experiments under consideration $\operatorname{Exp}_{17,549}^{\mathcal{E}_{C}, \text { NN:5-1-1 }}$ Identification of a specific broad scale experiment by its experiment number $(17,520)$. In addition, (for convenience) the associated error measure $\left(\mathcal{E}_{C}\right)$ and neural network architecture (NN:5-1-1) are specified

$\mathrm{S}_{i} \operatorname{Exp}_{j}$ Identification of a specific published experiment by its experiment series $(i=$ $\{1,2\}$ ) and experiment number $(j)$ within that series. 
$\mathrm{C}_{i}^{\text {exp }} \quad$ All objects such that $i=c_{\exp }$

$\mathrm{C}_{i}^{\text {obs }} \quad$ All objects such that $i=c_{o b s}$

$\mathcal{I} \quad$ An individual contains the encoding of a solution, $\mathcal{S}_{\beta}$, to a problem, $\mathbb{P}$ using a particular representation, $\mathcal{R}_{i}$

$\mathcal{I}_{\text {fit }} \quad$ The fitness associated to the individual $\mathcal{I}$

$\mathcal{P} \quad$ A population of individuals $(\mathcal{I})$

$\mathcal{P}=\left\{i=r \circ a, a \in \Gamma_{i}^{E C}\right.$ such that $\left.\mathcal{R}_{i}(s)=r, s \in \mathcal{S}_{\alpha}\right\}$ or, hopefully, $s \in \mathcal{S}_{\gamma \cap \beta}$

$\mathcal{P}^{\prime} \quad$ Another population (of individuals)

$\mathcal{A} \quad$ An archived population of individuals

$\mathcal{F}_{\mathcal{P}} \quad$ A family of populations $\left(\mathcal{F}_{\mathcal{P}}=\bigcup \mathcal{P}\right)$

$\mathcal{F}_{\mathcal{P}^{\prime}} \quad$ Another family of populations

$\Gamma^{E C}$ A set of parameters for an EC Algorithm

$\Gamma_{i}^{E C}$ A set of parameters associated with the $i^{\text {th }}$ individual.

$\Gamma_{i}^{E C}=\{$ parameters related to $\mathcal{I}\} \subseteq \Gamma^{E C}$

$\aleph_{i} \quad$ For a General Neural Network, the $i^{t h}$ neuron has $\aleph_{i}$ as its associated function

$o_{i} \quad$ For a General Neural Network, the $i^{\text {th }}$ neuron produces $o_{i}$ as its output

inDegree $\left(\aleph_{i}\right)$ For a General Neural Network, the number of inputs to the $i^{\text {th }}$ neuron outDegree $\left(\aleph_{i}\right)$ For a General Neural Network, the number of outputs from the $i^{\text {th }}$ neuron

$\operatorname{card}\left(\ell_{\text {wut }}\right)$ For a General Neural Network, this is the number of neurons that lead to output for the neural network (i.e. dimension of output vector). 
$\aleph_{j}^{\ell_{\text {out }}}$ For a Neural Network organized into $i$ layers, $\aleph_{j}^{\ell_{\text {out }}}$ represents the function associated with a specific neuron in the network output layer (i.e. layer $i$ ) $\left(\aleph_{j}^{\ell_{\text {out }}}\right.$ is equivalent to $\left.\aleph_{j}^{\ell_{i}}\right)$

$o_{j}^{\ell_{\text {out }}}$ For a Neural Network organized into $i$ layers, $o_{j}^{\ell_{\text {out }}}$ is the $j^{\text {th }}$ neuron's output in the output layer of the network ( $o_{j}^{\ell_{\text {out }}}$ is equivalent to $o_{j}^{\ell_{i}}$ )

$\delta_{i}^{\aleph} \quad$ Indication as to whether the output for the $i^{\text {th }}$ output layer neuron $\left(o_{i}^{\ell_{\text {out }}}\right)$, is expected to be excited. $\delta_{i}^{\aleph}=1$ when $i=c_{\text {exp }}$; otherwise $\delta_{i}^{\aleph}=0$

$\varphi_{j} \quad$ For a Neural Network organized into $i$ layers, $\varphi_{j}$ is a neuron's output from the last hidden layer in the network. (See $N D A)\left(\varphi_{j}\right.$ is equivalent to $o_{j}^{\ell_{i-1}}$ )

$\aleph_{j}^{\ell_{i}} \quad$ For a Neural Network organized into layers, $\aleph_{j}^{\ell_{i}}$ represents the function associated with the neuron in the $j^{\text {th }}$ position in the $i^{\text {th }}$ layer

$o_{j}^{\ell_{i}} \quad$ For a Neural Network organized into layers, $o_{j}^{\ell_{i}}$ represents the output of the neuron in the $j^{\text {th }}$ position within the $i^{\text {th }}$ layer

NN:5-1-1 Example of architecture specification for a Feed Forward Neural Network (FFNN) organized into 3 layers with 5 neurons in the first layer, 1 neuron in the hidden layer and 1 neuron in the output layer.

$f_{k} \aleph_{j}^{\ell_{i}}$ For a Neural Network organized into layers, $f_{k} \aleph_{j}^{\ell_{i}}$ represents the $k^{\text {th }}$ function associated with the $j^{\text {th }}$ neuron in the $i^{\text {th }}$ layer

$f_{k} o_{j}^{\ell_{i}} \quad$ For a Neural Network organized into layers, $f_{k} o_{j}^{\ell_{i}}$ represents the output of the $k^{\text {th }}$ function associated with the $j^{\text {th }}$ neuron in the $i^{\text {th }}$ layer

$\mathcal{E} \quad$ is used to refer to an error measure. The particular error measure will be a qualified form of $\mathcal{E}$.

$\mathcal{R}$ is used to refer to a rank. That is, an ordering.

$\mathcal{E}_{C} \quad=\frac{\sum_{i=1}^{c}\left(\operatorname{card}\left(\mathrm{C}_{i}^{\exp }\right)-\operatorname{card}\left(\mathrm{C}_{i}^{\exp } \cap \mathrm{C}_{i}^{\mathrm{obs}}\right)\right)}{\operatorname{card}(\mathrm{Obj})}$ 
$\mathcal{E}_{C_{N}}=\frac{\sum_{i=1}^{c}\left(\operatorname{card}\left(\mathrm{C}_{i}^{\exp }\right)-\operatorname{card}\left(\mathrm{C}_{i}^{\exp } \cap \mathrm{C}_{i}^{\text {obs }}\right)\right) / \operatorname{card}\left(\mathrm{C}_{i}^{\exp }\right)}{c}$

$\mathcal{E}_{C}^{T r} \quad$ That is, $\mathcal{E}_{C}$ restricted to training data only.

$\mathcal{E}_{C}^{T e} \quad$ That is, $\mathcal{E}_{C}$ restricted to testing data only.

$\mathcal{E}_{C_{N}}^{T r} \quad$ That is, $\mathcal{E}_{C_{N}}$ restricted to training data only.

$\mathcal{E}_{C_{N}}^{T e} \quad$ That is, $\mathcal{E}_{C_{N}}$ restricted to testing data only.

$\mathcal{E}_{C}\left({ }_{f_{i}} \aleph_{*}^{\ell_{\text {out }}}\right)$ For example, $\mathcal{E}_{C}$ could be computed on $f_{2} \aleph_{1}^{\ell_{\text {out }}}$ or ${ }_{f_{1}} \aleph_{>1}^{\ell_{\text {out }}}$

$\mathcal{E}_{C}^{T r}\left({ }_{f_{i}} \aleph_{*}^{\ell_{o u t}}\right)$ That is, $\mathcal{E}_{C}\left({ }_{f_{i}} \aleph_{*}^{\ell_{o u t}}\right)$ restricted to training data only.

$\mathcal{E}_{C}^{T e}\left({ }_{f_{i}} \aleph_{*}^{\ell_{\text {out }}}\right)$ That is, $\mathcal{E}_{C}\left({ }_{f_{i}} \aleph_{*}^{\ell_{\text {out }}}\right)$ restricted to testing data only.

$\mathcal{E}_{C_{N}}\left(f_{i} \aleph_{*}^{\ell_{\text {out }}}\right)$ For example, $\mathcal{E}_{C_{N}}$ could be computed on ${ }_{f_{2}} \aleph_{1}^{\ell_{\text {out }}}$ or ${ }_{f_{1}} \aleph_{>1}^{\ell_{\text {out }}}$

$\mathcal{E}_{C_{N}}^{T r}\left({ }_{f_{i}} \aleph_{*}^{\ell_{\text {out }}}\right)$ That is, $\mathcal{E}_{C_{N}}\left({ }_{f_{i}} \aleph_{*}^{\ell_{\text {out }}}\right)$ restricted to training data only.

$\mathcal{E}_{C_{N}}^{T e}\left({ }_{f_{i}} \aleph_{*}^{\ell_{\text {out }}}\right)$ That is, $\mathcal{E}_{C_{N}}\left({ }_{f_{i}} \aleph_{*}^{\ell_{\text {out }}}\right)$ restricted to testing data only.

$\overline{\mathcal{E}^{T r}} \quad$ Average error on training data for a particular measure.

For example, $\mathcal{E}_{C}^{T r}\left({ }_{f_{i}} \aleph_{*}^{\ell_{\text {out }}}\right)$ or $\mathcal{E}_{C_{N}}^{T e}\left({ }_{f_{i}} \aleph_{*}^{\ell_{\text {out }}}\right)$

$\overline{\mathcal{E}^{T e}} \quad$ Average error on testing data for a particular measure.

For example, $\mathcal{E}_{C}^{T r}\left({ }_{f_{i}} \aleph_{*}^{\ell_{\text {out }}}\right)$ or $\mathcal{E}_{C_{N}}^{T e}\left({ }_{f_{i}} \aleph_{*}^{\ell_{\text {out }}}\right)$

$\overline{\mathcal{E}_{\mathcal{R}}^{T r}} \quad$ Average rank on training data for a particular measure.

$\overline{\mathcal{E}_{\mathcal{R}}^{T e}} \quad$ Average rank on testing data for a particular measure.

$$
\begin{aligned}
& f_{1} \aleph_{1}^{\ell_{\text {out }}}=\left|c \cdot \frac{2}{\pi} \cdot \operatorname{asin}\left(\sin \left(\pi \cdot x-\frac{\pi}{2}\right)\right)\right| \\
& f_{2} \aleph_{1}^{\ell_{\text {out }}}=\frac{c}{\left(1+e^{-x}\right)} \\
& { }_{f_{3}} \aleph_{1}^{\ell_{\text {out }}}=\frac{c}{2} \cdot \sin (c \cdot \pi \cdot x)+\frac{c}{2} \\
& f_{4} \aleph_{1}^{\ell_{\text {out }}}=\frac{2}{\pi} \cdot \operatorname{atan}(x)
\end{aligned}
$$




$$
\begin{aligned}
& f_{1} \aleph_{>1}^{\ell_{\text {out }}}=\left\{c_{i}: i \in\left\{1,2, \cdots, c=\operatorname{card}\left(\ell_{\text {out }}\right)\right\} \text { and } o_{i}^{\ell_{\text {out }}}=\max _{1 \leq j \leq c}\left(o_{j}^{\ell_{\text {out }}}\right)\right\} \\
& f_{2} \aleph_{>1}^{\ell_{\text {out }}}=\left\{c_{i}: i \in\left\{1,2, \cdots, c=\operatorname{card}\left(\ell_{\text {out }}\right)\right\} \text { and }\left(\sum_{j=1}^{c}\left|\frac{1}{1+e^{-o_{j}^{\ell_{0 u t}}}}-\delta_{i}^{\aleph}\right|\right)<\varepsilon\right\}
\end{aligned}
$$




\section{Abstract}

A neural network classifier or non-linear discriminant analysis network is sought. Classical network neurons are aggregations of a weight multiplied by an input value and then controlled via an activation function; with the addition of a bias node within each network's layer. This thesis learns everything within the neuron using a variant of Genetic Programming called Gene Expression Programming (including the connections between neurons) for a fixed number of layers and a fixed number of neurons per layer. That is, this thesis does not explicitly use weights or activation functions within a neuron, nor does it construct an explicit bias node. More precisely, this thesis claims that "weights and activation functions do not need to be specified explicitly when learning the functions associated to neurons within a neural network, as is done in the classical case". Promising results are reported for the 1 and 2 class cases. For example, the investigated one class problem is for the determination of underground caves, in which, for some measuring locations, cave membership is known and for others it is not. The multiple class cases are harder and deserve further investigation, but, even so, some good results have been published. 


\section{Acknowledgements}

I would like to thank Professor Tony White at Carleton University for supervising me and for correcting my many mistakes. I very much appreciate him giving me the academic freedom to explore and discover; indeed, it was a pleasure to discuss academic topics. In addition, I would like to thank Julio J. Valdés at the National Research Council for many things; he encouraged me to pursue higher learning while instructing me in Science.

I would like to thank Alfredo Vellido from the SOCO Group of the Polytechnic University of Catalonia in Spain; for his support of the Magnetic Resonance Spectra research. In addition I gratefully acknowledge the former INTERPRET (EU-IST1999-10310) European project partners. Data providers: Dr. C. Majós (IDI), Dr. A. Moreno-Torres (CDP), Dr. F.A. Howe and Prof. J. Griffiths (SGUL), Prof. A. Heerschap (RU), Dr. W. Gajewicz (MUL) and Dr. J. Calvar (FLENI); data curators: Dr. A.P. Candiota, Ms. T. Delgado, Ms. J. Martín, Mr. I. Olier and Mr. A. Pérez (all from GABRMN-UAB). Prof. Carles Arús, GABRMN group leader.

I would like to thank Lluis Belanche for providing the Spitzbergen (Werenskiold) data and to thank William H. Wolberg for depositing the breast cancer data within the UCI machine learning repository.

I would like to thank everyone in the Knowledge Discovery Group, at the Institute for Information Technology (IIT) in the National Research Council Canada (NRC); including Bob Orchard, Fazel Famili, and Marvin Zaluski. I would also like to thank the technical support team at NRC-IIT; especially Marc Léveillé, Bart Breedyk, and Charles Kingsley. Also, Dan Gamache from the Visual Information Technology Group at IIT. I also very much appreciate the Cerebrovascular Research Group, at the Institute for Biological Sciences (IBS) in NRC; in particular, Arsalan Haqqani and John Kelly. 


\section{Chapter 1}

\section{Introduction}

But our beginnings never know our ends! - Portrait of a Lady 1.97 (1911)

Thomas Stearns Eliot ${ }^{1}$ (1888-1965)

This thesis ${ }^{2}$ claims that "weights and activation functions do not need to be specified explicitly when learning the functions associated to neurons within a neural network, as is done in the classical case". In addition, the following minor claims are made: i) a global optimizer (i.e. a variant of Genetic Programming called Gene Expression Programming) is useful for discovering previously unknown neuron functions within such a network, ii) the choice of neural network output layer mapping function (and consequently number of output layer neurons) plays a large role in determination of the quality of the obtained models, and iii) distributed computing was essential in order to facilitate $\approx 2$ years of required single CPU computation.

\subsection{Problem Statement}

A problem that exists is to learn a feed forward artificial neural network (FFNN) for classification or general function approximation purposes given training and testing data. The classical approach requires neurons within the network to have a bias node, weights associated to each neuron and an activation function applied to the neuron output. The learning problem addressed within this thesis is to construct a FFNN for classification by relaxing the constraints imposed by the classical solution by learning the complete real-valued function for each neuron. This thesis constrains the neural network architecture to a pre-specified, heuristically chosen, number of layers and number of neurons per layer. However, the connections between neurons from layer $i$

\footnotetext{
${ }^{1}$ T.S. Eliot won the 1948 Nobel Prize in Literature.

${ }^{2}$ This thesis follows a particular research methodology, namely, first full facts, then interpretation and strives for thoroughness, completeness and impartiality [Chamberlin, 1890].
} 
to layer $i+1$ is not constrained. The general function approximation problem is left for future research.

\subsection{Motivation for Pursuing this Research}

One estimate of a human brain's properties states that it contains 100 billion neurons (each neuron producing 1,000 pulses per second) with 100 trillion synapses (connections between neurons) and the capability of storing 100 million megabytes [Moravec, 1998]. Such a massively parallel information processing device, such as the biological neural network, has inspired the creation of artificial neural networks (ANNs) which are used in order to gain more understanding of their biological counterparts or are used for solving artificial intelligence problems.

Much biological research work has been performed. For example, biological neural networks have been observed through the use of light to monitor the activity of a large number of individual synapses simultaneously [Miesenbock and Rothman, 1997] In addition, Miesenböck [Wells, 2007] uses light to control ${ }^{3}$ a neural network. For example, he has caused headless Drosophila to fly and is pursuing research in order to understand how the nervous system encodes behavior. Historically, biological research into neural networks struggled to overcome the problem of determining whether the network was composed of discrete units or a continuous network ${ }^{4}$ to either align itself with cell theory [Mazzarello, 1999] or not. After 50 years of assembling all of the essential data [Fodstad, 2001], the Neuron Doctrine ${ }^{5}$, with four tenets was proposed:

1. fundamental structural and functional unit of the nervous system is the neuron

2. neurons are discrete cells (in alignment with Cell Theory [Mazzarello, 1999])

\footnotetext{
${ }^{3}$ [Wells, 2007] uses a physicists approach, in that simplification, abstraction, and synthesis, are most important. Miesenböck also stresses the idea that in order to establish causality and dissect a complex mechanism it is essential to be able to control it.

${ }^{4}$ Vilhelm von Waldeyer (1891) stated that the nervous system consists of innumerable units that are not connected anatomically and he proposed to call the unit neuron from the Greek word for sinew [Fodstad, 2001].

${ }^{5}$ Many pioneers including 3 nobel laureates were involved in studying the nervous system. In particular, Spanish anatomist Santiago Ramón y Cajal (1852-1934), on the basis of the histological techniques developed by Italian anatomist Camillo Golgi (1843-1926) both received the 1906 Nobel Award in Medicine and Physiology. Norwegian Arctic explorer Fridtjof Nansen received the award for Peace in 1922. [Fodstad, 2001]
} 
3. the neuron is composed of 3 parts - the dendrites, axon and cell body, and

4. information flows along the neuron in one direction (from the dendrites to the axon, via the cell body).

On the other hand, artificial neural networks have been used, for example, to infer a function from observational data, since they have been proven to be universal function approximators [Hornik, 1989, Hornik, 1993] ${ }^{6}$. Of course there are many techniques for constructing functions from data. In fact, the concept of function originated with Galileo ${ }^{7}$ (1564-1642), and his observations of motion data [Malik, 1980]. Such a plethora of techniques for direct discovery of general analytic functions is due to the infinite size of the search space and the enormous challenges it entails. An investigation of all possible techniques (e.g. for Classical Exploratory Data Analysis (a.k.a. Data Mining) [Tukey, 1977], for foundations and fundamental concepts of mathematics [Eves, 1997], for a history of knowledge [van Doren, 1991] and for a general historical account [Wells, 2006]) is not possible.

The classical ANNs have very good performance. However, their interpretation is awkward for those not familiar with the intricacies. On the other hand, analytic functions are i) easier to understand by humans, ii) the preferred building blocks of modeling, and iii) a classical and highly condensed form of knowledge. As such, a hybrid combining the desirable features of classical ANNs and classical analytic functions is sought. It is this statement that motivates the research reported in this thesis.

Not only does biology inspire the construction of the concept of ANN, but it can inspire how such an ANN is trained. In 1858, Charles Darwin and Alfred Russel Wallace both (independently) proposed natural selection as the origin of new phenotypic variants - observable as opposed to unobservable (genotypic) - and hence, new species of organisms [Kutschera and Niklas, 2004]. Such a biological theory of evolution, and how populations of individuals change through time, has undergone changes and in

\footnotetext{
${ }^{6}$ When using arbitrary squashing functions, ANNs can approximate virtually any function of interest to any desired degree of accuracy, provided sufficiently many hidden units are available [Hornik, 1989].

${ }^{7}$ The finger of Galileo. Nature. Vol. 424. July 2003 p.17
} 

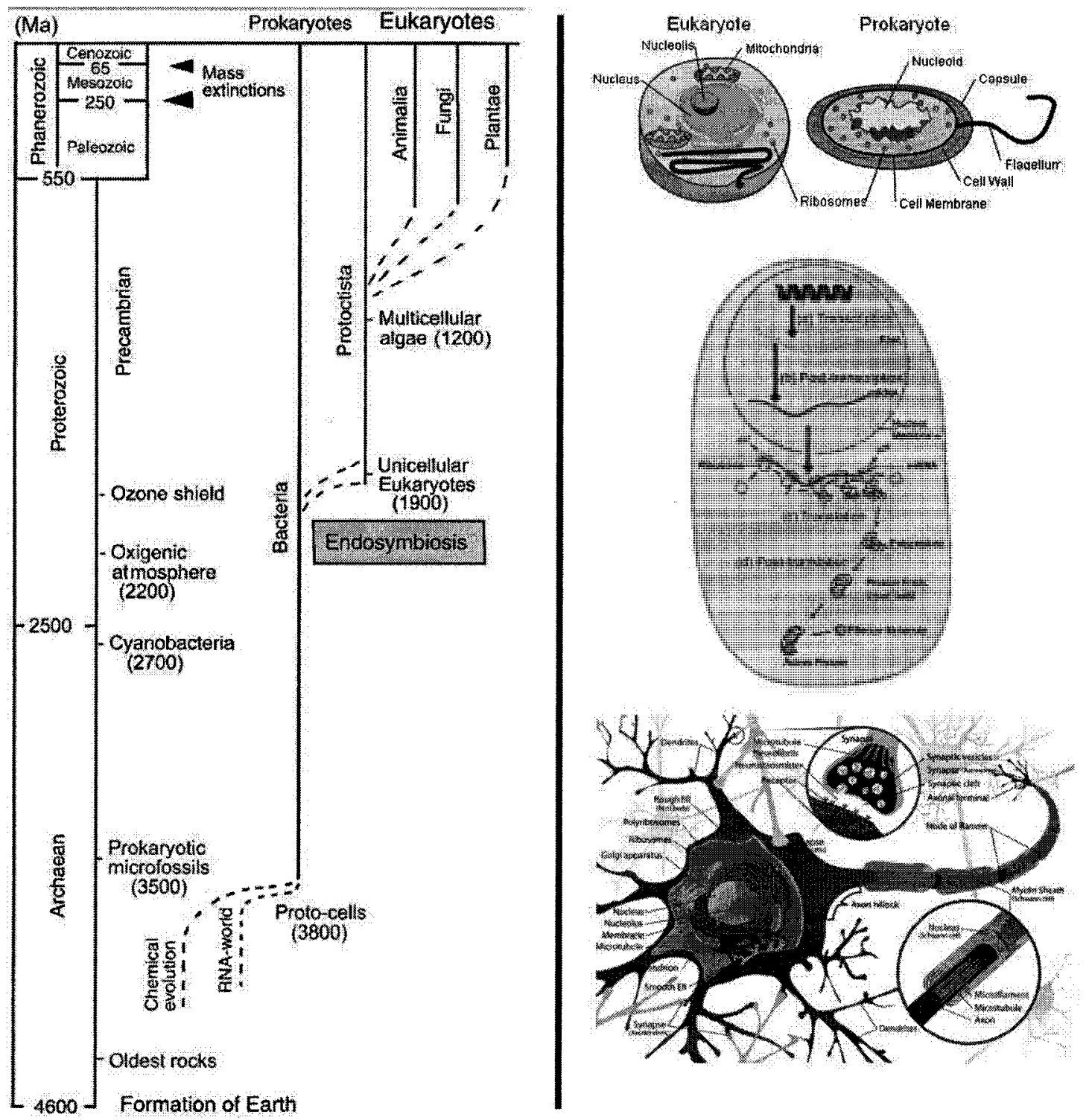

Figure 1.1: Left: Chronology of biological events: i) formation of Earth, ii) chemical soup complexity increasing with prevolution [Nowak and Ohtsuki, 2008], iii) start of replication (i.e. life) with evolution, $i v$ ) extreme diversification of cells for single and multiple celled organisms. Right: Examples of different cell types and their complex internal processes; neuron cell at bottom right.

Images from: [Kutschera and Niklas, 2004], [NCBI, 2008] and NIH Publication No. $05-1051$. 
the 1930s, Darwinian-Wallace natural selection was combined with Mendelian inheritance [Wright, 1931] to form the modern definition [Kutschera and Niklas, 2004] in which the connection between the units of evolution (genes) and the mechanism of evolution (natural selection) was made. This theory attempts to explain the history of life on Earth [Wald, 1954] and Fig. 1.1 on page 4 shows a compressed representation of the fossil record and a Tree Of Life relating how life might have emerged from a basic chemical soup that exhibits prevolution [Nowak and Ohtsuki, 2008] ${ }^{8}$ and shows that the result of evolution - pre-life selection and mutation plus the addition of replication - has currently yielded numerous types of life in the form of many variants of cells (e.g. neurons) that are composed of many internally complex processes ${ }^{9}$, for example, DNA [Crick, 1968]. Of course, groups of cells also undergo evolution, such as increasing brain size [Oxnard, 2004] and both cells or groups of cells (tissues, organs, individuals, etc.) are well adjusted (fitted) to their respective environments.

However, environments are not static, they are dynamic changing things that require organisms to continually adapt ${ }^{10}$ during which hybrid traits appear. For example, the Archaeopteryx (Fig. 1.2 on page 6) was a very classical dinosaur/bird that was thought to be the missing link relating the evolution of dinosaurs into birds. Thus, this concept of populations of individuals with generally increasing fitness ${ }^{11}$ that are more able to adapt to their respective environment can inspire computational methods. In particular, if populations of ANNs are subjected to an evolutionary process, called Evolutionary Computation, then increasingly better solutions are learned.

\subsection{Objectives}

The objectives are:

\footnotetext{
${ }^{8}$ Life is that which replicates and evolves. The origin of life is also the origin of evolution. Prevolutionary dynamics have selection and mutation, but no replication... Life marches in with the ability of replication. [Nowak and Ohtsuki, 2008]

${ }^{9} \mathrm{Sex}$ is an important process; each organism can produce $2^{N}$ different sperm or egg cells leading to $4^{N}$ different offspring genotypes. If $N=150$ genes, then $2^{N} \approx 10^{45}$ and $4^{N} \approx 10^{90} \ldots$ close to the total number of particles in the observable universe [Kutschera and Niklas, 2004]

${ }^{10}$ Adaptation is different from natural selection [Orr, 2005]

${ }^{11}$ Gradual adaptation has an alternative view; punctuated equilibria, in which sudden changes occur, followed by long periods of "statis" [Eldredge and Gould, 1972]
} 


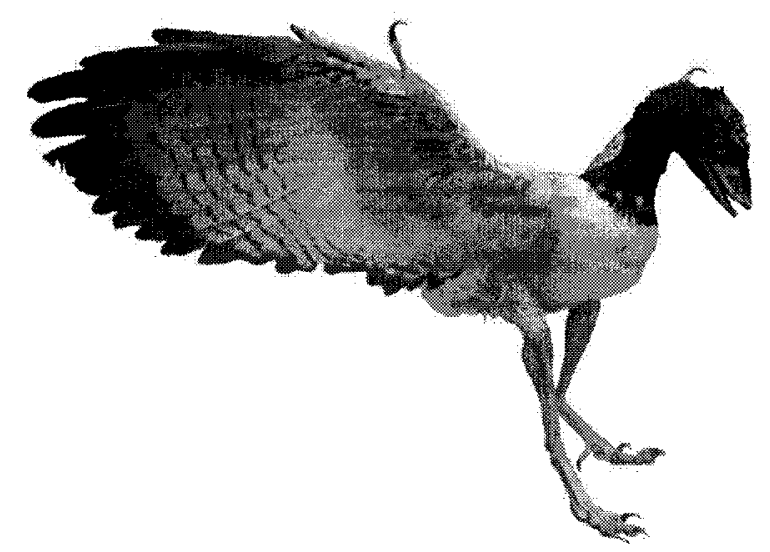

Figure 1.2: Picture of an Archaeopteryx model at the Oxford University Museum: a missing link in biological evolution relating dinosaurs and birds. This biological hybrid inspires computational-based hybrids [Wikipedia, 2008a].

1. Develop a technique for data mining, incorporating biologically plausible principles.

2. Implement the technique using sound software engineering principles.

3. Evaluate the technique using multiple data sets and sound scientific principles of data analysis.

\subsection{Research Statement}

The goal of this research is to infer a useful function (or functions) from observational data. In particular, to represent functions as analytical expressions within a neural network structure and to determine effects of changing the structure with respect to:

complexity of constructed functions (i.e. expression trees) in terms of attribute use and basis function use,

errors associated to the constructed functions in terms of:

- classification error (and ranked errors) on training and testing data,

- normalized classification error (and ranked errors) on training/testing data,

- mapping error for non-linear discriminant analysis (NDA) networks. 
selection of controlling algorithm parameters in terms of the quality of the results (i.e. errors).

In particular, for a neural network based on a variant of genetic programming (gene expression programming), do experimental results suggest that one constructed representation is superior (in some objective sense) over the others? A comparison of the suggested approach with classical and/or frequently used methods would give an indication of the possible potential applicability.

\subsection{Overview of Results}

The contributions of this thesis are:

1. Development of hybrid neural network architecture for function approximation.

2. Development of a biologically-inspired representation for these neural networks.

3. Development of training algorithms for (1) and (2) based upon principles of Evolutionary Computation.

4. Experimental assessment of hybrid neural networks using real world data sets.

In particular, two types of experiments are reported; 3 broad scale and 1 published. For the series of broad scale experiments 3 data sets are used; cave data (Insunza), breast cancer data from UCI, and water sample data (Werenskiold). All training and testing error distributions are reported along with statistical tests for all pairwise selections. In addition, for a selected network output mapping function $\left(f_{1} \aleph_{>1} \ell^{\text {out }}\right)$ that performs the best, the parameter spaces are investigated by the construction of 3D representations of the 11D parameter space (See Table 6.6 on page 104 for the 11 selected parameters). The parameter spaces for the 3 broad scale results (See Section 7.2 on page 134) show that the Neural Network based on Gene Expression Programming Algorithm NN-GEP found solutions for all combinations of controlling parameters. It is interesting that the good solutions are located in different regions of the parameter spaces. This led to a heuristic approach for the use of NN-GEP in which a broad sweep of the parameter space is performed first, followed by a 
focussed search around any good solutions. Published work using this heuristic on a 200 dimensional, 4 class problem was reported for Magnetic Resonance Spectra, in which NN-GEP was able to perform better than Rough Sets and an Individual Dichotomization technique, which was used as a baseline.

\subsection{Organization of Thesis}

This thesis is organized into chapters. The first chapter after this initial introductory chapter is the background chapter in which initial considerations are listed; including considering the domain, the algorithm, the search methodology, and the internal and external representations. This is then followed by a discussion of the specific algorithms used by first specifying a general evolutionary computation algorithm and then the specific algorithms; including the Genetic Algorithm, the Genetic Programming Algorithm, the Gene Expression Programming Algorithm, and the Neural Network Algorithm. Other background information is included about multi-objective optimization, Rough Sets, an Individual Dichotomization Algorithm and the concept of regression and non-parametric statistics. The third chapter performs a broad sweep of the literature in search of state of the art material related to neural networks. Work on such things as classical neural networks with weights and activation functions all the way up to possible encodings of neural networks within evolutionary computation algorithms, and even further, to related literature on classification measures and the multiple class problem.

The fourth chapter is the heart of the thesis because the theory behind the NNGEP Algorithm is presented starting from a simple motivating example. The representation of a neural network, the fitness of an Individual encoding a neural network, the algorithm controlling parameters, the termination criteria and enhancements are discussed. This is then followed by specific software engineering and design details within the fifth chapter, in which class and sequence diagrams for the implementation are presented.

The sixth chapter describes all 4 data sets used within this thesis. The broad scale methodology is also described and the following chapter reports the results of the experiments. A discussion of the investigated broad scale parameter space for 
NN-GEP is made along with empirical comparisons with other techniques. Published results are reported for Magnetic Resonance Spectra data and how the results compare with Rough Sets and a baseline algorithm named Individual Dichotomization.

In the concluding eighth chapter results are summarized, conclusions are drawn and possible future directions are posited.

Supporting items are listed as appendices to this thesis; including an example configuration file, available symbols and the experimental databases containing all of the results. The reader need not access this additional material in order to understand the main body of the thesis. However, it is strongly recommended that the reader consult the notation section in the front inaterial of this thesis before reading Chapter 4 on page 62 , Chapter 6 on page 90, Chapter 7 on page 109 or Chapter 8 on page 151; basically, the notation should be consulted before reading anything after the theory chapter describing NN-GEP.

\subsection{Chapter Summary}

The main thesis claim, that "weights and activation functions do not need to be specified explicitly when learning the functions associated to neurons within a neural network, as is done in the classical case" was made. A broad discussion of neural networks from both the biological and artificial perspectives was made. The thesis research statement was stated and an overview of all of the main results were presented. Finally, the organization of the forthcoming thesis was described; with the next chapter providing a starting point for understanding the main concepts within the thesis. 


\section{Chapter 2}

\section{Background}

Any sufficiently advanced technology is indistinguishable from magic.

Arthur C. Clarke (born 1917)

This chapter provides material for more clearly understanding the contents of the rest of the thesis. Some brief initial considerations are described related to the data, the algorithm, and the results. A general evolutionary computation algorithm is specified in which three instances are described: the Genetic Algorithm (GA), the Genetic Programming Algorithm (GP) and the Gene Expression Programming Algorithm (GEP). In addition, the Artificial Neural Network Algorithm (ANN) is described along with other techniques that are used. Some material has been excluded from this chapter and is either within the nomenclature reported at the beginning of the thesis, or is within the appendices.

\subsection{Before Constructing an Algorithm: Initial Considerations}

Various aspects should be considered before deciding upon an algorithm that will be applied for a specific domain or domains. For example, should the algorithm be very specifically tailored to the domain or should a more general approach be taken? Several considerations will be very briefly discussed in this section, with secondary issues omitted in the interests of brevity. For example, the following will be discussed: i) representation $i$ ) algorithms, and iii) operators for manipulation of representation.

\subsubsection{The Domain and its Data}

There are both domain specific issues based on the theory within a particular domain (e.g. The Theory of Biological Evolution) and domain independent issues for which, perhaps, a general theory might be constructible (e.g. Evolutionary Computation). 
Each domain is different. Some domains have very reliable, clean data while others have high degrees of uncertainty and noise. Issues from the following domains are considered throughout this thesis:

Biological Data, such as Magnetic Resonance Spectra from Brain Cancer samples [Barton and Valdés, 2008] and clinical data from Breast Cancer samples [Wolberg and Mangasarian, 1990], [Asuncion and Newman, 2007],

Geophysical Prospecting Data, such as from Insunza Cave measurements for learning about the presence of an underground cave [Valdés and Barton, 2006],

Hydrochemical Data, such as from Werenskiold Water samples for learning about global climate changes [Valdés and Barton, 2007].

\subsubsection{The Algorithm}

There are at least two approaches towards constructing an algorithm: i) construct the algorithm and then determine its applicability upon different domains, or ii) be involved in a particular domain that has a specific problem that needs to be solved and construct the algorithm based on generalizing the solution within the domain. For (ii), more than one domain may be considered simultaneously and, hence, a potentially more general algorithm encompassing all of the essential details may be constructed.

\subsubsection{The Search Methodology}

The algorithm will be searching a space for a global or a local solution. The search will be for an exact solution or for an approximation to the exact solution. The search can be guided by heuristics to regions of the search space that are more promising. The search methodology can also be based upon populations of solutions or individuals. Considerations regarding operators that are used for manipulation of one of more solutions concurrently are important. 


\subsubsection{The Internal Representation}

A representation $\left(\mathcal{S}_{\beta}\right)$ of a solution must be chosen in such a way that the algorithm can compute with it. This, then, dictates the potential input that is able to be obtained and any potential pre-processing that might be necessary (e.g. discretization).

\subsubsection{The Results (The External Representation)}

The presentation of the solution that is represented within the computer and found by the algorithm also needs to be considered [Joma, 1988]. In other words, the internal representation $\left(\mathcal{S}_{\alpha}\right)$ of a solution $(\mathcal{S})$ and the reporting of that solution as the output of the algorithm $(\mathbb{S})$ need not necessarily be the same. Such an explicit consideration of the algorithm's results is of concern for practical reasons (e.g. Human-Computer Interaction, or see [Valdés and Barton, 2007]).

\subsection{Foundation Algorithms}

A specification of each algorithm that is necessary to understand the material presented within this thesis is provided. A general evolutionary algorithm with particular examples, such as genetic algorithms and genetic programming along with neural networks are presented. Other algorithms from the field of Evolutionary Computation, Machine Learning or Pattern Recognition such as those from Swarm Intelligence, or perhaps Grammatical Evolution, or Evolution Strategies, or Differential Evolution, etc. will not be discussed.

\subsubsection{A General Specification for Evolutionary Computation}

Biological evolution with its many complex interrelated processes ${ }^{1}$ (Fig. 2.1 on page 13, Fig. 2.2 on page 13) has inspired computational evolution. It was first proposed in [Eldredge and Gould, 1972] that evolution does not necessarily occur gradually, but, rather, has long periods of stasis followed by a sudden change. A general Evolutionary Computation (EC) algorithm (Alg. 1 on page 14) consists of

\footnotetext{
${ }^{1}$ selection vs. adaptation [Orr, 2005]... fitness vs. selection (short term or long term? for individual or population?)... etc.
} 


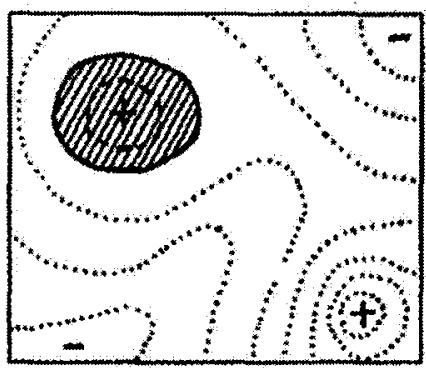

A. Increased Mutation or reduced Selection $4 N U, 4 N S$ very large

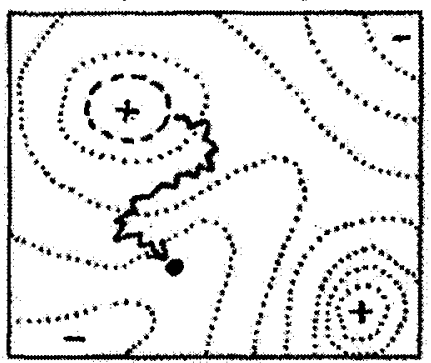

D. Close Inbreeding $4 N U, 4 N S$ very small

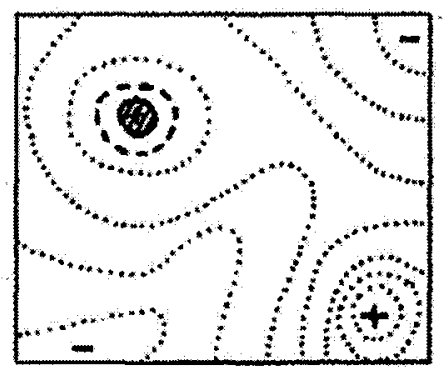

B. Increased Selection or reduced Mutation 4NU, 4NS very larqe

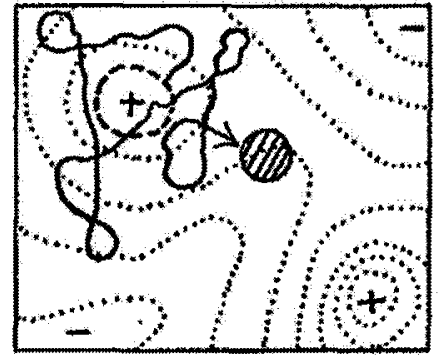

E. Slight Inbreeding $4 N O, 4 N 5$ medium

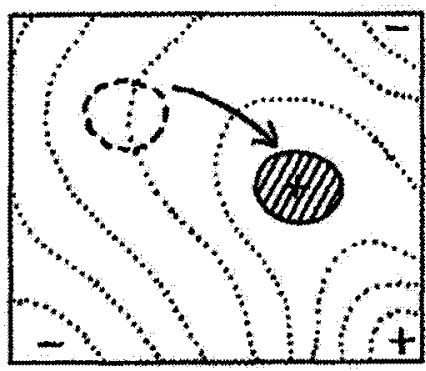

C. Qualitative Change of Environment 4NU, 4 NS very lerge

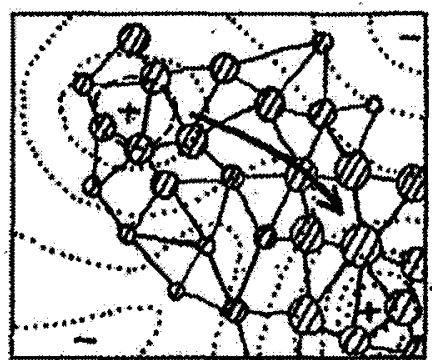

F. Division into local Races $4 \mathrm{~nm}$ medium

Figure 2.1: Many factors affect populations of individuals [Wright, 1932]: if gene mutation too high, then freaks are obtained, not evolution; selection must not be too high; too close inbreeding leads to extinction; crossbreeding is beneficial.

Compare with Fig. 2.2 on page 13.

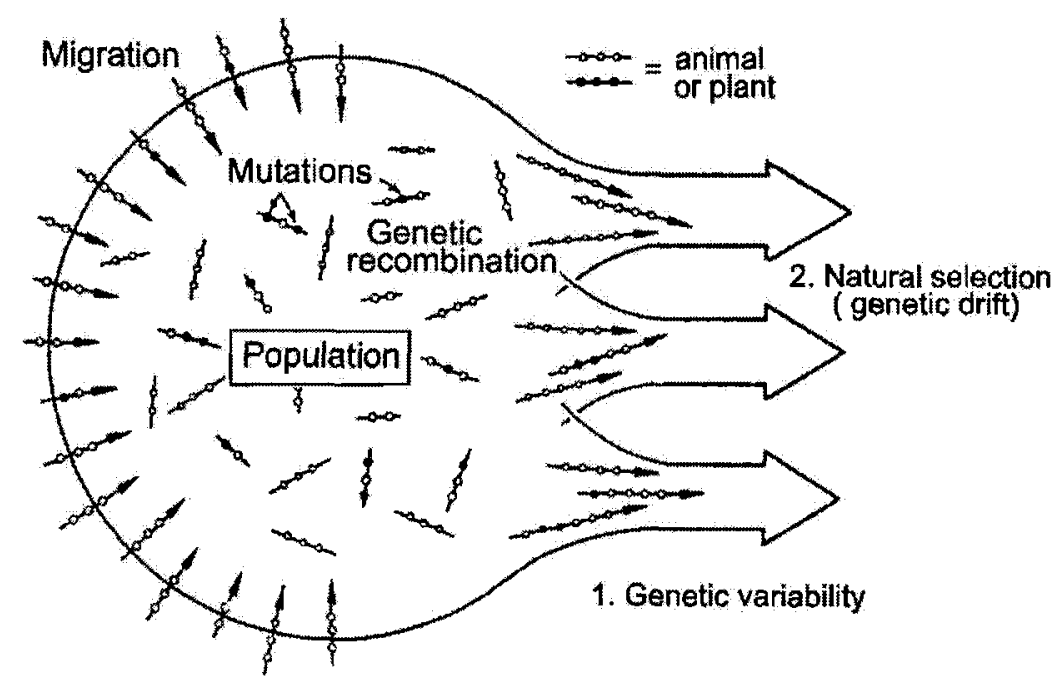

Figure 2.2: Relationship between basic processes that bring about phenotypic changes in a population of organisms (animals or plants) [Kutschera and Niklas, 2004]. Life involves selection, mutation and replication [Nowak and Ohtsuki, 2008].

Compare with Fig. 2.1 on page 13. 
a problem, $\mathbb{P}$, as input and a set of solutions, $\mathbb{S}$, as output. Alg. 1 on page 14 consists of three main stages: i) initialization, ii) processing generations, and iii) post-processing.

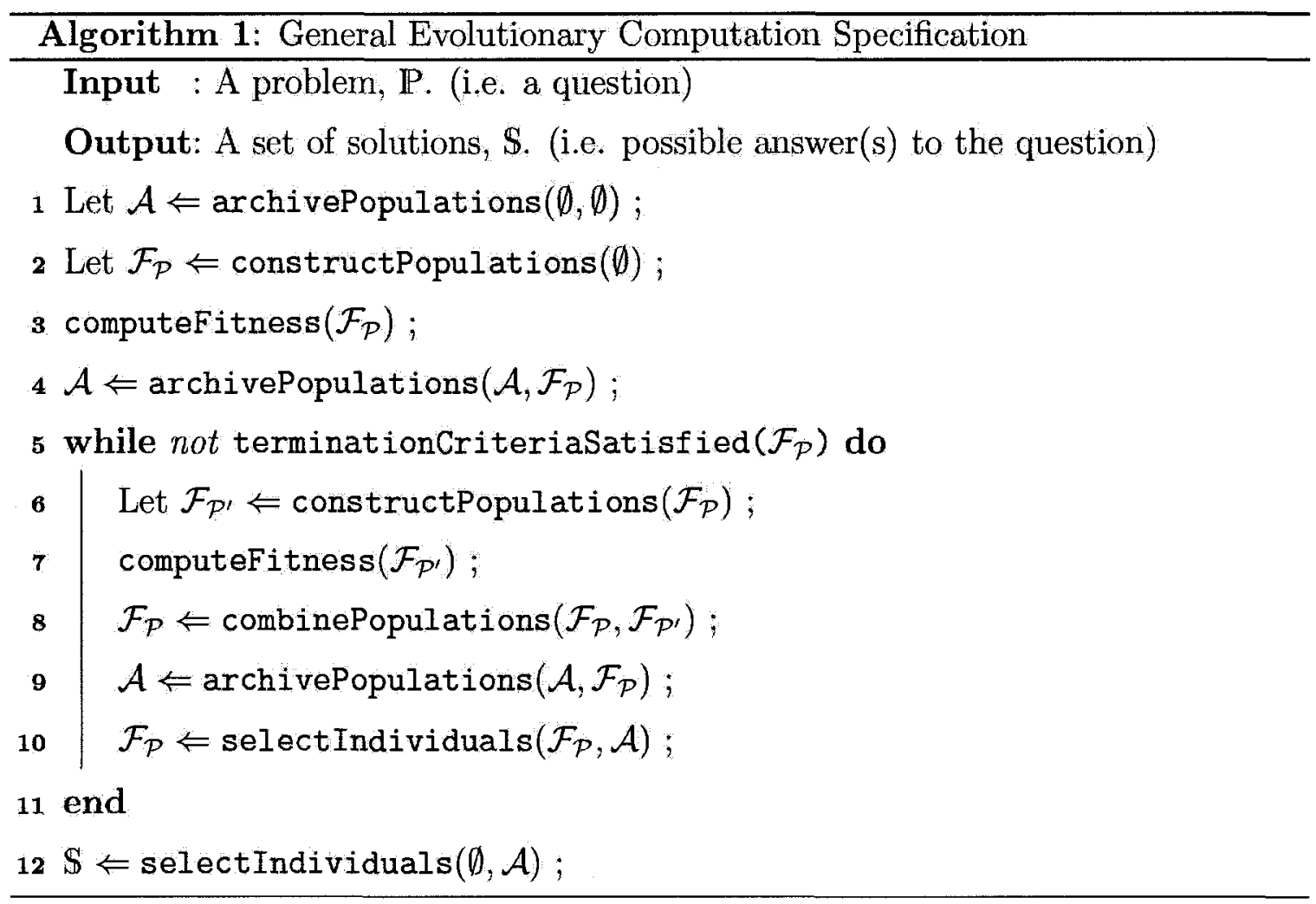

Initialization Line 1-4: On Line 1 an archive $\mathcal{A}$ of individuals $\mathcal{I}$, is created, which, in general, may or may not be used by a particular instance of an EC algorithm. Then, on Line 2 an initial family of populations of individuals, $\mathcal{F}_{\mathcal{P}}$, where each individual, $\mathcal{I}$, represents an encoding of an initial computable solution guess, $\mathcal{S}_{\beta}$, for $\mathbb{P}$ is constructed. A particular EC algorithm can construct one population, $\mathcal{F}_{\mathcal{P}}=\{\mathcal{P}\}$, or many populations, $\mathcal{F}_{\mathcal{P}}=\left\{\mathcal{P}_{1}, \mathcal{P}_{2}, \ldots, \mathcal{P}_{n}\right\}$. For example, the construction can use $i$ ) domain knowledge (i.e. specific background knowledge about the problem), ii) some kind of heuristic(s) about placement of individuals (solutions) throughout the initial solution space, or iii) pre-computed solutions from another algorithm, etc. The algorithm continues, on Line 3, by computing the fitness of all of the individuals $(\mathcal{I} \in \mathcal{P})$ contained in all of the populations $\left(\mathcal{P} \in \mathcal{F}_{\mathcal{P}}\right)$; each $\mathcal{I}$ can have one or more fitness values associated with it. 
Processing a Generation Line 5-11: On Line 5 all algorithm termination criteria are checked on all $\mathcal{I} \in \mathcal{F}_{\mathcal{P}}$. If the algorithm should stop (terminate) then Line 12 will be executed, else Line 6-10 will be executed. One generation is considered to have occurred when one pass through the loop has completed. A generation begins with the construction of a new family of populations of individuals $\mathcal{F}_{\mathcal{P}^{\prime}}$ based on the current family of populations of individuals $\mathcal{F}_{\mathcal{P}}$ by EC operators such as crossover, mutation, etc and then proceeds to the calculation of the fitness of each newly created individual (old individuals do not need to have their fitness values recalculated if the environment is static) in $\mathcal{F}_{\mathcal{P}^{\prime}}$. At this point, a current family of populations $\mathcal{F}_{\mathcal{P}^{\prime}}$ and a previous family of populations $\mathcal{F}_{\mathcal{P}}$ exist, from which we can combine them in some manner on Line 8 by, for example, non-dominated sorting. Line 9 continues the current generation processing by archiving some individuals for later recall. For example, elitism ${ }^{2}$ could be considered as a form of archiving within main memory, or, as another example, writing particular individuals to the hard drive. It is not necessary that a particular EC algorithm perform archiving, but at least elitism, which helps convergence, is wise. The next family of populations, $\mathcal{F}_{\mathcal{P}}$, for consideration in the next generation is then selected based on the previous family of populations, $\mathcal{F}_{\mathcal{P}}$ and the current archive of individuals, $\mathcal{A}$.

Post-processing Line 12-13: After termination criteria have been satisfied, Line 5, for the current family of populations $\mathcal{F}_{\mathcal{P}}$ (and by implication the current archive $\mathcal{A})$, Line 12 will select the fittest individuals to report as solutions $\mathbb{S}$ to the problem $\mathbb{P}$ that was posed.

The general evolutionary computation algorithm (Alg. 1 on page 14) addresses the issues raised in Section 2.1 on page 10. The algorithm can be applied to many domains because it can be tailored via its computation of fitness for an individual (Line 3,7) in order to include domain dependant information. In fact, these fitness functions are used within the search methodology (Line $4,5,9,10)$ in order to determine whether

\footnotetext{
${ }^{2}$ The best individual(s) are considered to be the elite individuals. Elitism means that the best individuals are always remembered by the algorithm. More specifically, in generation $i$ the $x$ best individuals are immediately a part of generation $i+1$.
} 
an exact or approximate solution is desired from the family of populations of individuals $\mathcal{F}_{\mathcal{P}}$ (Line $2,8,9,10$ ) or $\mathcal{F}_{\mathcal{P}^{\prime}}$ (Line 6,8). The internal representation of an individual is the data that a fitness function can access in order to compute the fitness. Alg. 1 on page 14 performs operations on this internal representation (Line $2,6,8$ ) that create new internal representations. The internal representation that is returned from Alg. 1 on page 14 on Line 12 will lead to the external representation that will be presented as the algorithm's solution to the particular problem of interest.

\subsubsection{A Genetic Algorithm}

John H. Holland carefully considering the following learning problem: "given a previous sample of behaviors which have been differentially rewarded, what behaviors should next be generated to optimize expected rewards?" [Hayes-Roth, 1975]. Holland's solution was stated that learning should be considered as a general problem of adaptation to the environment. In particular, the following informal questions were considered within his formalism [Holland, 1992, page 29]:

1. To what parts of its environment is the organism ${ }^{3}$ adapting?

2. How does the environment act upon the adapting organism?

3. What structures are undergoing adaptation?

4. What are the mechanisms of adaptation?

5. What part of the history of its interaction with the environment does the organism retain in addition to that summarized in the structure tested?

6. What limits are there to the adaptive process?

7. How are different (hypotheses about) adaptive processes to be compared?

This section briefly presents John Holland's artificial system specification.

\footnotetext{
${ }^{3}$ system, organization, ...
} 


\subsubsection{Internal Representations}

Holland developed a formal framework and associated analysis [Holland, 1992] by first defining a set of structures ${ }^{4}$ that must be searched for an optimal structure that is well adapted to the environment through the use of a set of detectors over the structures. The general evolutionary computation algorithm in Alg. 1 on page 14 uses Holland's structures on lines (Line $\mathbf{2 , 6 , 8}$ ), and names them internal representations. The most common realization of one of the following representations (for practical use within a computer) is the binary string (e.g. 010101000001011011110001).

Detectors An internal representation is built as a set of $l$ detectors (e.g. indicator variables), where two structures are distinguishable only insofar as they have distinct representations. The representation for $\mathcal{I}$ is: $\left(\delta_{1}\left(\mathcal{S}_{\alpha}\right), \delta_{2}\left(\mathcal{S}_{\alpha}\right), \cdots, \delta_{l}\left(\mathcal{S}_{\alpha}\right)\right)$ where $\delta_{i}: \mathcal{S}_{\alpha} \rightarrow\{0,1\}$ [Holland, 1992, p.66].

Allele Linkage The internal representation is extended to allow functional meaning of an allele to not be dependant on position. The extended representation for $\mathcal{I}$ is: $\left(\left(1, \delta_{1}\left(\mathcal{S}_{\alpha}\right)\right),\left(2, \delta_{2}\left(\mathcal{S}_{\alpha}\right)\right), \cdots,\left(l, \delta_{l}\left(\mathcal{S}_{\alpha}\right)\right)\right)$. Then, for example, a different representation for $\mathcal{I}$ is: $\left(\left(2, \delta_{2}\left(\mathcal{S}_{\alpha}\right)\right),\left(1, \delta_{1}\left(\mathcal{S}_{\alpha}\right)\right), \cdots,\left(l, \delta_{l}\left(\mathcal{S}_{\alpha}\right)\right)\right)$ [Holland, 1992, p.106].

Dominant Allele The internal representation is extended to allow extra alleles to be carried in unexpressed form (a reservoir of protected alleles). As one simple dominance incarmation, a pair of alleles is maintained (one is dominant and one is recessive corresponding to Fisher's 1937 theory ${ }^{5}$ ) along with a dominance map indicating which allele is dominant; $\delta_{i}^{\text {dom }}: \mathcal{S}_{\alpha} \rightarrow\left\{1,1_{0}, 0\right\}$, with $1_{0}$ being the recessive allele whenever it is paired with 0 . The extended representation for $\mathcal{I}$ is:

$$
\begin{aligned}
& \mathcal{I}=\left(\mathcal{I}_{1}, \mathcal{I}_{2}\right) \text { with } \mathcal{I}_{1}=\left(\left(1, \delta_{1}^{\text {dom }}\left(\mathcal{S}_{\alpha}\right)\right),\left(2, \delta_{2}^{\text {dom }}\left(\mathcal{S}_{\alpha}\right)\right), \cdots,\left(l, \delta_{l}^{\text {dom }}\left(\mathcal{S}_{\alpha}\right)\right),\right) \text { and } \\
& \mathcal{I}_{2}=\left(\left(1, \delta_{1}^{\text {dom }}\left(\mathcal{S}_{\alpha}\right)\right),\left(2, \delta_{2}^{\text {dom }}\left(\mathcal{S}_{\alpha}\right)\right), \cdots,\left(l, \delta_{l}^{\text {dom }}\left(\mathcal{S}_{\alpha}\right)\right),\right) .[\text { Holland, 1992, p.112]. }
\end{aligned}
$$

\footnotetext{
${ }^{4}$ Each structure is considered to be representable $\left(\mathcal{S}_{\alpha}\right)$.

${ }^{5}$ Fisher's theory of dominance has been refuted [Orr, 1991] in favour of Wright's theory, but that does not detract from Holland's representation.
} 
Detector Adaptation Detectors can be added or modified as needed. That is, the number of detectors can change and the subroutine specifying a particular detector may change ${ }^{6}$. [Holland, 1992, p.117,141].

\subsubsection{Operators}

The operations on the internal representation of an individual are used in order to construct new internal representations. This is called the search methodology, and in Alg. 1 on page 14 refers to (Line $4,5,9,10$ ). Holland's operators are:

Crossing-over Choose $\mathcal{I}_{a}=a_{1} \cdot a_{2} \cdots a_{l}$ and $\mathcal{I}_{b}=b_{1} \cdot b_{2} \cdots b_{l}$ and choose a number $x \in\{1,2, \cdots, l-1\}$. Then two new structures $\left(\mathcal{I}_{a}^{\prime}\right.$ and $\left.\mathcal{I}_{b}^{\prime}\right)$ are formed: $\mathcal{I}_{a}^{\prime}=a_{1} \cdot a_{2} \cdots a_{x}, b_{x+1}, b_{x+2}, \cdots, b_{l}$ and $\mathcal{I}_{b}^{\prime}=b_{1} \cdot b_{2} \cdots b_{x}, a_{x+1}, a_{x+2}, \cdots, a_{b}$. [Holland, 1992, p.98]

Inversion Choose $\mathcal{I}=a_{1} \cdot a_{2} \cdots a_{l}$ and choose two numbers $x_{1}^{\prime}, x_{2}^{\prime} \in\{0,1,2, \cdots, l+1\}$. Define $x_{1}=\min \left\{x_{1}^{\prime}, x_{2}^{\prime}\right\}$ and $x_{2}=\max \left\{x_{1}^{\prime}, x_{2}^{\prime}\right\}$. Then the new (inverted) structure $\left(\mathcal{I}^{\prime}\right)$ is formed via inversion of the segment to the right of position $x_{1}$ and to the left of position $x_{2}: \mathcal{I}^{\prime}=a_{1} \cdot a_{2} \cdots a_{x_{1}} \cdot a_{x_{2}-1} \cdot a_{x_{2}-2} \cdots a_{x_{1}+1} \cdot a_{x_{2}} \cdots a_{l}$. [Holland, 1992, p.107]

Mutation Choose $\mathcal{I}=a_{1} \cdot a_{2} \cdots a_{l}$ and choose $h$ positions $x_{1}, x_{2}, \cdots, x_{h}$ (independently of each other) for mutation. Then the new (mutated) structure $\left(\mathcal{I}^{\prime}\right)$ is: $\mathcal{I}^{\prime}=a_{1} \cdot a_{2} \cdot a_{x_{1}-1} \cdot a_{x_{1}}^{\prime} \cdot a_{x_{1}+1} \cdots a_{x_{2}-1} \cdot a_{x_{2}}^{\prime} \cdot a_{x_{2}+1} \cdots a_{l}$ where $a_{x_{j}}^{\prime}$ is drawn at random from the range of the detector $\delta_{i}$ corresponding to position $x_{j}$. [Holland, 1992, p.109]

Dominance-change The aforementioned operators can be extended to afford the dominant allele concept within the representation. The dominance-change operator is straightforward, the dominance (or recessiveness) of an allele is changed [Holland, 1992, p.111].

\footnotetext{
${ }^{6}$ The modifiability of detectors seems to be inspired by GP?
} 


\subsubsection{A Genetic Programming Algorithm}

Genetic Programming (GP) was introduced in [Koza, 1989], [Koza, 1992] and is an extension of the Genetic Algorithm to specifically evolve a population of computer programs $^{7}$. In Alg. 1 on page 14, the family of populations of individuals $\mathcal{F}_{\mathcal{P}}$ (Line $2,8,9,10$ ) or $\mathcal{F}_{\mathcal{P}^{\prime}}$ (Line 6,8) is usually a family composed of one set. In other words, $\mathcal{F}_{\mathcal{P}}$ and $\mathcal{F}_{\mathcal{P}^{\prime}}$ are each a simple set of individuals $\mathcal{I}$. GP programs can be symbolic expression trees, which, ultimately, are functions. In [Koza, 1989] it is stated that: In hierarchical genetic algorithms, the individuals in the population are LISP S-expressions (i.e. rooted point-labeled trees in a plane), instead of linear character strings. The set of similar individuals sharing common features (i.e. the schemata) is a hyperspace of LISP S-expressions (i.e. rooted point-labeled trees in a plane) sharing common features.

\subsubsection{Internal Representation}

The general evolutionary computation algorithm in Alg. 1 on page 14 uses Koza's computer programs on lines (Line $\mathbf{2 , 6 , 8}$ ), and names them internal representations. More specifically, one of Koza's computer programs is called an S-expression where S stands for symbolic, and is used, for example, within such programming languages as the List Processing Language (LISP). The following S-expression, which uses prefix notation,

$$
\left(* V_{1}\left(+V_{1} V_{2}\right)\right)
$$

is equivalent to the following equation using infix notation:

$$
V_{1} *\left(V_{1}+V_{2}\right)
$$

In [Koza and Rice, 1991] they give an example of a LISP-S expression that represents a neural network with 2 inputs $\left(V_{1}, V_{2}\right), 2$ outputs and the following function set $F=$ $\{P, W,+,-, *, \%\}$, with $P$ being a linear threshold function, $W$ being multiplication

\footnotetext{
${ }^{7} \mathrm{~A}$ computer program is understood as an entity that receives inputs, performs computations which transform these inputs and produces some output in a finite amount of time.
} 
for weights, and \% a protected division ${ }^{8}$ :

$$
\begin{aligned}
(\operatorname{LIST} & \left(\mathrm{P}\left(\mathrm{W} V_{2}-1.423\right)\left(\mathrm{W} V_{1}(+1.20 .4)\right)\right. \\
& \left.\left(\mathrm{P}\left(\mathrm{W} V_{1}(*-1.7-0.9)\right)\left(\mathrm{W} V_{1}(-1.10 .5)\right)\right)\right)
\end{aligned}
$$

The root of the tree is always a LIST function. The number of arguments of LIST equals the number of output signals. This is the only time the LIST function is used in the S-expression. Then, the rules of construction require that the function at the level of the tree immediately below the LIST function must be linear threshold processing functions $P$.

\subsubsection{Operators}

The operations on the internal representation of an individual are used in order to construct new internal representations. This is called the search methodology, and in Alg. 1 on page 14 refers to (Line $4,5,9,10)$. Koza's operators must preserve the structure of S-expressions and he has two classes of operator: primary and secondary, which are:

\subsubsection{Primary Operator: Crossover (sexual recombination)}

Koza also calls this operator slicing and dicing. It operates by randomly and independently selecting one point in each parent using a uniform probability distribution [Koza, 1989, p.101]. This crossover operation is well defined for any two S-expressions and any two crossover points and the resulting offspring are always valid LISP Sexpressions. Offspring contain some traits from each parent. Viewed in terms of lists in LISP, the crossover fragment is the sub-list starting at the crossover point.

\subsubsection{Primary Operator: Reproduction}

The reproduction operator is asexual [Koza, 1992, p.99] because it only operates on one parental S-expression and produces only one offspring S-expression. First, an

\footnotetext{
$8_{\text {i.e. }} \frac{x}{0}$ will result in 0 being returned
} 
S-expression is selected ${ }^{9}$ based on fitness. Second, the selected is copied, without alteration, from the current population to the new population.

\subsubsection{Secondary Operator: Mutation}

Mutation is asexual an operates on only one parental S-expression [Koza, 1992, p.106]. It begins by selecting a point at random within the S-expression. This mutation point can be an internal (i.e. function) point or an external (i.e. terminal) point of the tree. The mutation operation then removes whatever is currently at the selected point and whatever is below the selected point and inserts a randomly generated subtree at that point. This operator is controlled by a parameter that specifies the maximum size (measured by depth) for the newly created subtree. This typically has the same value as the parameter for the maximum initial size of S-expressions in the initial random population.

\subsubsection{Secondary Operator: Permutation (Inversion)}

The permutation operator is a generalization of the GA inversion operator [Koza, 1992, p.107]. Permutation is asexual, and begins by selecting a function (internal) point of the LISP S-expression at random. If the function at the selected point has $k$ arguments, a permutation is selected at random from the set of $k !$ possible permutations. The arguments are then permuted according to the selected permutation. The function at the selected point can be commutative, in which case no immediate change of the S-expression value will occur (i.e. no change in fitness).

\subsubsection{Secondary Operator: Editing}

Editing is asexual [Koza, 1992, p.108] and recursively applies a pre-established set of domain independent an domain specific editing rules to each S-expression in the population. For example, $(+12)$ will be replaced by 3 and (AND True True) will be replaced by (True).

\footnotetext{
${ }^{9}$ There are many selection methods based on fitness; popular is fitness-proportionate selection[Koza, 1992, p.99]
} 


\subsubsection{Secondary Operator: Encapsulation}

Encapsulation is asexual [Koza, 1992, p.110] and is a means for automatically identifying a potentially useful subtree and giving it a name so that it can be referenced and used later. The operator begins by selecting a function (internal) point of the LISP S-expression at random. The result is one offspring S-expression and one new subtree definition.

For example, if point 3 (multiplication) is selected in $(+A(* B C))$ then a new function called $E 0$ will be created with body $(* B C)$ and the original S-expression will be replaced with $(+A(E 0))$.

\subsubsection{Secondary Operator: Decimation}

For some complex problems, the distribution of fitness values over the initial random population will be skewed so that a very large percentage of the individuals have very poor fitness [Koza, 1992, p.112]. The decimation operator has 2 parameters, a percentage and a condition in which the operator should be invoked. For example, only $10 \%$ of the population will be kept and the rest will be deleted when the decimation operator is applied at the end of the first generation.

\subsubsection{A Gene Expression Programming Algorithm}

Gene expression programming (GEP) [Ferreira, 2001], [Ferreira, 2006a] like genetic algorithms (GAs) and genetic programming (GP), is an evolutionary algorithm as it uses populations of individuals, selects them according to fitness, and introduces genetic variation using one or more genetic operators.

GEP can be considered to be a variant of GP of which there are many approaches (and implementations). A discussion about all of their relative merits, drawbacks and properties is beyond the scope of this thesis. The key distinction, however, lies in the biological definition of evolution [Kutschera and Niklas, 2004]:

1. geneticists define it as "irreversible changes of the genetic composition of populations" and focus attention on the genotype, and 
2. naturalists define it as "gradual descent with modification (inclusive of the diversification of species)" and focus attention on the phenotype.

Since, it is claimed [Ferreira, 2006b], that GAs and GPs focus on the genotype and that GEP focuses on the phenotype. This thesis does not address the validity of this claim, but does investigate the experimental performance of GEP using specific internal representations and operators. This thesis uses Ferreira's experimentally found default values for GEP and investigates several other combinations.

\subsubsection{Internal Representation}

GEP individuals are nonlinear entities of different sizes and shapes (expression trees) encoded as strings of fixed length [Ferreira, 2001]. GEP uses an unambiguous translation system to transfer the language of chromosomes into the language of expression trees and vise versa. The structural organization of GEP chromosomes allows a functional genotype/phenotype relationship, as any modification made in the genome always results in a syntactically correct ET or program. The set of genetic operators applied to GEP chromosomes always produces valid ETs.

Detailed examples are presented in [Ferreira, 2006a]. The algebraic expression:

$$
\frac{\sqrt{a+b}}{c-d}
$$

can also be represented as a diagram or expression tree (ET) as in Fig. 2.3 on page 23, which is the phenotype of GEP chromosomes.

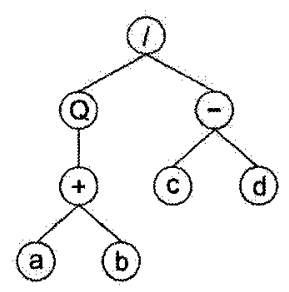

Figure 2.3: Example of a GEP Expression Tree (ET) from [Ferreira, 2006a]. "Q" represents the square root function.

The genotype can be inferred from the expression tree and is:

01234567

/Q-+cdab 
which is a reading of the ET from left to right and from top to bottom. Eq. 2.5 on page 23 is called an open reading frame (ORF) that always starts at position 0 ("/"), which is the start codon and terminates at position 7 (" $\mathrm{b}$ "), which is the stop $\operatorname{codon}^{10}$. These ORFs are called K-expressions from GEP's Karva language.

GEP genes have a head and a tail. The head contains symbols that represent functions and terminals. The tail can only contain terminals. Therefore, two different alphabets occur at different regions within a gene. For a particular problem, the length of the head $h$ is chosen, which then determines the length of the tail by the following relation:

$$
t=h(n-1)+1
$$

where $n$ is the function within the head that has maximum arity ${ }^{11}$.

For example, consider a gene with the set of terminals $T=\{a, b\}$ and the set of functions $F=\{Q, *, /,-,+\}$ (thus, $n=2$ ). If $h$ is chosen so that $h=10$, then $t=10(2-1)+1=11$ and then the length of the gene will be $h+t=10+11=21$.

The following gene has the head on the left and the tail on the right ${ }^{12}$ :

$$
\begin{aligned}
& 012345678901234567890 \\
& +* / a-Q b b / * \text { aabaabbabaa }
\end{aligned}
$$

The gene in Eq. 2.7 on page 24 codes for the expression tree in Fig. 2.4 on page 25.

If a mutation occurs a position 3 , changing the " $a$ " into " + ", then the gene in Eq. 2.8 on page 24 is obtained with the enlarged expression tree in Fig. 2.5 on page 25. A different inutation may cause the ET to shrink.

$$
\begin{aligned}
& 012345678901234567890 \\
& +* /+-Q b b / * \text { aabaabbabaa }
\end{aligned}
$$

\footnotetext{
${ }^{10} \mathrm{I}$ have added the stop codon into the representation reported by [Fetreira, 2006a] in order to be more clear. However, its location is not known until after ET construction.

${ }^{11}$ A function's arity is the number of arguments of the function.

${ }^{12} \mathrm{~T}$ have changed the representation that is used in [Ferreira, 2006a]. In particular, $I$ have added a box around the tail, rather than making it bold. I have also introduce the stop codon symbol into the representation even though its position is not known until after ET construction.
} 


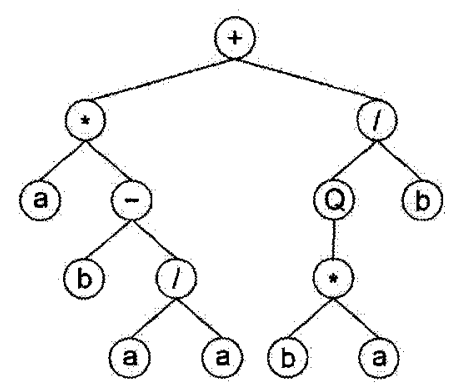

Figure 2.4: Example of a GEP Expression Tree (ET) for a gene [Ferreira, 2006a].

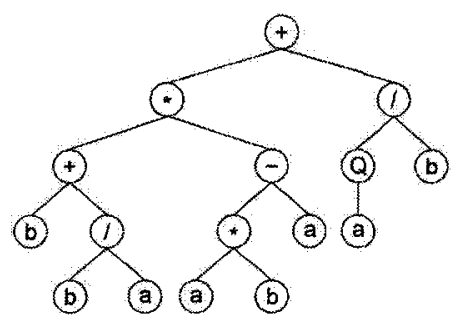

Figure 2.5: Example of a GEP Expression Tree (ET) for a gene after mutation [Ferreira, 2006a].

The chromosomes in GEP can be composed of more than one gene of equal length. For a particular run, the number of genes and the head size $h$ are selected. An example of a chromosome's genome of length 39 composed of 3 genes, each of length 13 is shown in Table 2.1 on page 25, with the corresponding ET shown in Fig. 2.6 on page 26. The final ET could be linearly encoded as the K-expression in Eq. 2.9 on page 25 of length 24 .

$$
\begin{aligned}
& 012345678901234567890123 \\
& ++* * / \mathrm{Q}-\mathrm{Q}-\mathrm{aQ} / \mathrm{b} * \mathrm{~b} / \mathrm{ababbbbb}
\end{aligned}
$$

According to [Ferreira, 2006a], GP handles random numerical constants by using a special terminal named "ephemeral random constant". For each ephemeral random constant used in the trees of the initial population, a random number of a special

Table 2.1: Example of a 3 gene GEP Chromosome. The 3 stop codon locations are not known until after ET construction, but are shown for clarity. The middle gene's stop codon occurs in the head, while the other two stop codon's occur in the tails.

$$
\begin{aligned}
& 012345678901201234567890120123456789012 \\
& * \mathrm{Q}-\mathrm{b} / \mathrm{a} \text { bbbaaba /aQb-b bbaabaa } * \mathrm{Q}-/ \mathrm{b} * \text { abbbbaa }
\end{aligned}
$$



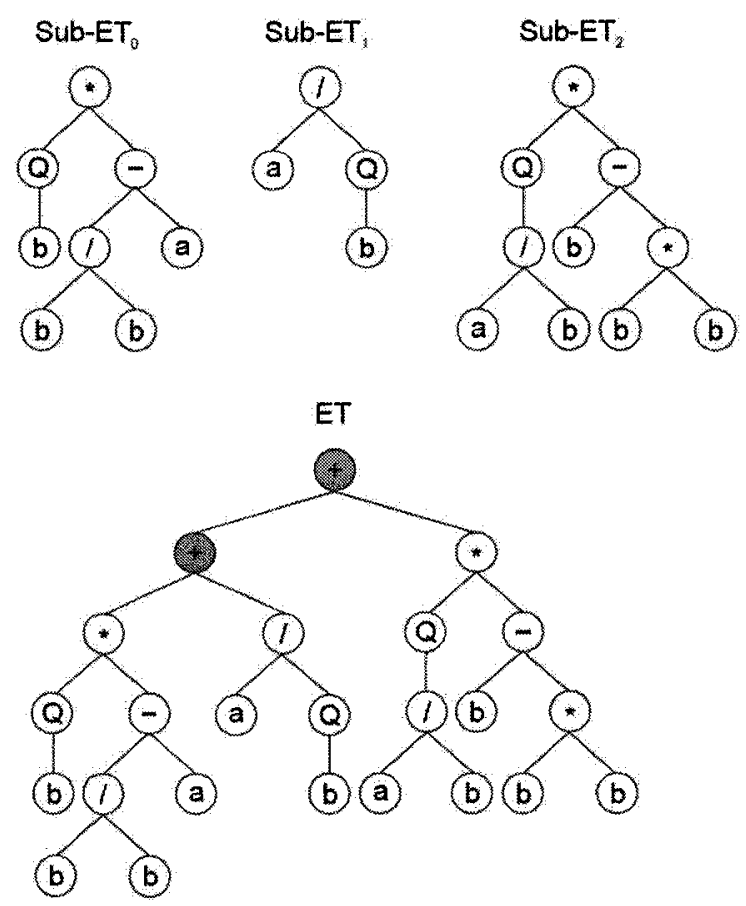

Figure 2.6: Example of a GEP Expression Tree (ET) for 3 genes [Ferreira, 2006a]. The top three sub-ETs correspond to each gene. The bottom ET is the result of posttranslational linking with addition (linking functions are shown in gray).

data type in a specified range is generated. Then these random constants are moved around from tree to tree by the crossover operator. It is claimed [Ferreira, 2006a] that with this method no new constants are created during evolution.

The head and the tail of a gene within GEP are known as domains. For GEP to handle constants an extra domain called Dc and an extra terminal "?" are used. Thus, for each gene, the initial random constants are generated during the creation of the initial population and kept in an array. However, a special operator is used to introduce genetic variation in the available pool of random constants by mutating the random constants directly. In addition, the usual operators of GEP (mutation, inversion, transposition, and recombination), plus a Dc-specific transposition and a Dc-specific inversion, guarantee the circulation of the random constants in the population. The Dc domain comes after the tail domain and has length $t$. For example, the single gene chromosome with $h=8$ is shown in Eq. 2.10 on page 27 
and has the associated ET shown in Fig. 2.7 on page 27.
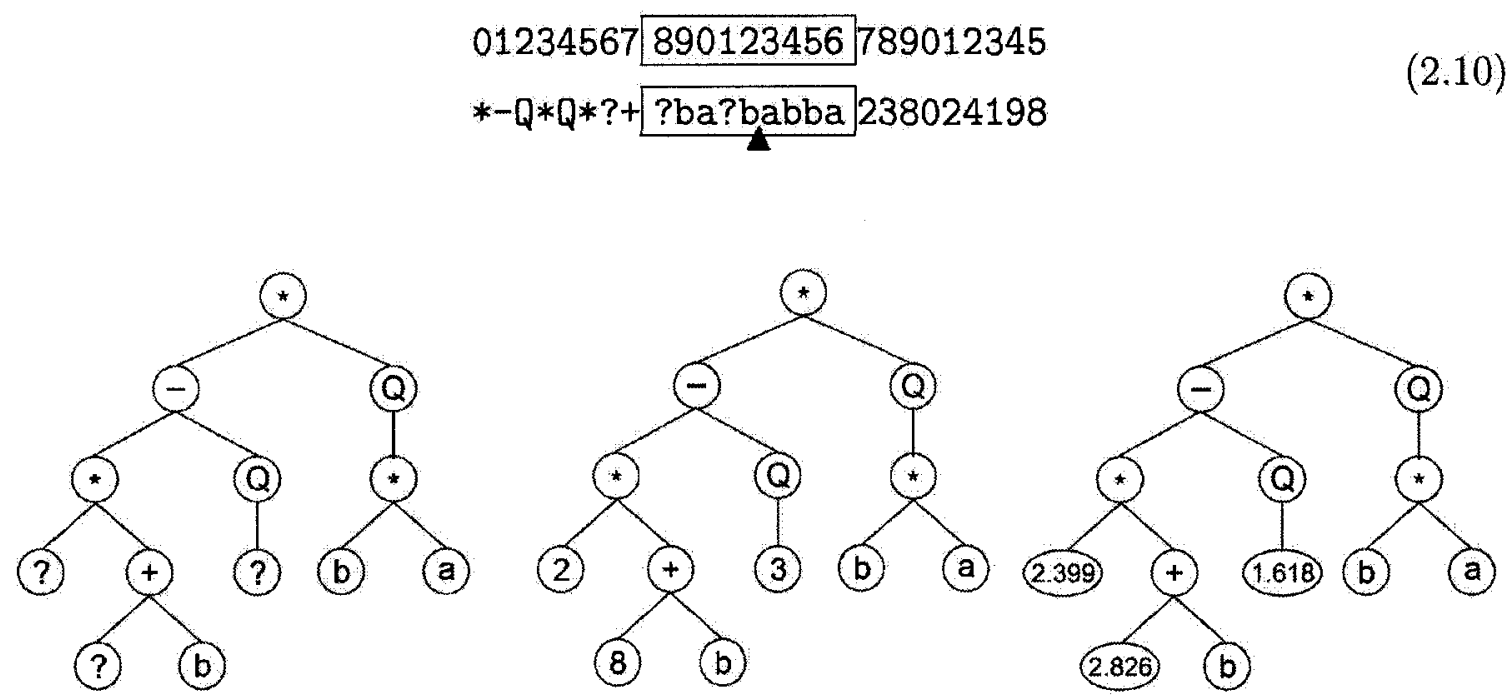

Figure 2.7: Example GEP ET with head, tail and Dc domains [Ferreira, 2006a]. Left ET shows the locations of the special terminals. Middle ET shows those terminals replaced with their respective indices into the array $A$ of random constants. If $A=\{1.095,1.816,2.399,1.618,0.725,1.997,0.094,2.998,2.826,2.057\}$, then the resulting $\mathrm{ET}$ is on the right.

\subsubsection{Operators}

The descriptions of the GEP operators are Dr. Ferreira's.

Inversion is restricted to the heads of genes. Any sequence might be randomly selected and inverted. The default value for the inversion rate is 0.1 . The inversion operator randomly chooses the chromosome, the gene to be modified, and the start and termination points of the sequence to be inverted.

Mutation can occur anywhere in the chromosome. But the structural organization of chromosomes must remain intact, that is, in the heads any symbol can change into another (function or terminal), whereas in the tails terminals can only change into other terminals. This way, the structural organization of chromosomes is preserved, and all the new individuals produced by mutation are structurally correct programs. The default value for the mutation rate is 0.044 . 
One point recombination the parent chromosomes are paired and split up at exactly the same point. The material downstream of the recombination point is afterwards exchanged between the two chromosomes. The default value for the one-point recombination rate is 0.3 .

Two point recombination two parent chromosomes are paired and two points are randomly chosen as crossover points. The material between the recombination points is afterwards exchanged between the parent chromosomes, forming two new daughter chromosomes. The default value for the two-point recombination rate is 0.3 .

Gene recombination entire genes are exchanged between two parent chromosomes, forming two daughter chromosomes containing genes from both parents. The exchanged genes are randomly chosen and occupy exactly the same position in the parent chromosomes. The default value for the gene recombination rate is 0.1 .

RIS transposition Root Insertion Sequence elements or RIS elements are short fragments with a function in the first position that transpose to the start position of genes. The default value for the RIS transposition rate is 0.1 . All RIS elements start with a function, and therefore must be chosen among the sequences of the heads. For that, a point is randomly chosen in the head and the gene is scanned downstream until a function is found. This function becomes the start position of the RIS element. If no functions are found, the operator does nothing.

IS transposition Any sequence in the genome might become an IS element and, therefore, these elements are randomly selected throughout the chromosome. A copy of the IS element is made and inserted at any position in the head of a gene, except the first position.

Gene transposition An entire gene works as a transposon and transposes itself to the beginning of the chromosome. In contrast to the other forms of transposition, in gene transposition, the transposon (the gene) is deleted at the place 
of origin. The default value for the gene transposition rate is 0.1 . The gene transposition operator randomly chooses the chromosome to be modified and then randomly chooses one of its genes (except the first) to transpose.

RNC mutation direct mutation of random numerical constants or RNC mutation for short, randomly selects particular targets in the arrays where the random numerical constants are kept, and randomly generates a new constant.

Dc mutation is similar to the mutation that occurs in the heads and tails of genes with a default rate of 0.044 .

Dc inversion randomly choose the chromosome, the gene to be modified, and the start and termination points of the sequence to be inverted.

De IS transposition randomly choose the chromosome, the start and termination points of the IS element, and the target site.

\subsubsection{An Artificial Neural Network (ANN) Algorithm}

This section describes the general neural network and the specific case of a feedforward neural network that is used within this thesis. The first model of a neuron then then described along with the classical artificial neural network learning algorithm in Section 2.2.5.6 on page 34 .

\subsubsection{General Neural Network}

There are many types of neural networks. Fig. 2.8 on page 30 is an example of a general neural network architecture. Phrased in terms of graph theory, nodes are neurons and directed edges indicate the excitement directionality relation. That is, those neurons that are capable of exciting a given neuron are the incoming edges and those neurons that a particular neuron is capable of exciting are the outgoing edges. In such a general neural network (subset of a complete graph) a neuron is capable of both exciting other neurons and exciting itself. To be more particular, Fig. 2.8 on page 30 shows two pieces of information per neuron: i) $\aleph_{i}(\cdot)$ is the function associated with a particular neuron, which has an arbitrary label such as $i$, 


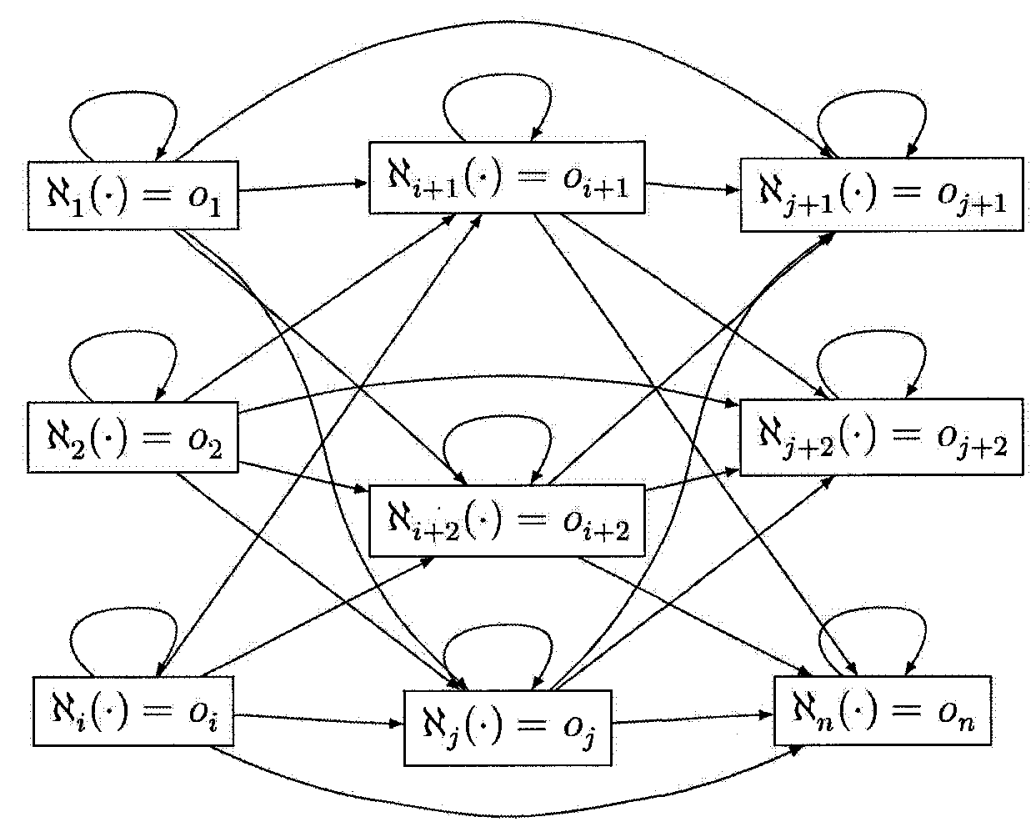

Figure 2.8: General Neural Network (a graph) containing $(n)$ neurons. Each box is a neuron in the network (that may or may not be organized into layers) where all activity occurs (e.g. activation, aggregation, etc). Weights are learned within the neuron. Not all possible edges are shown (i.e. for complete graph).

and $i i) o_{i}$ is the output of the function associated to the $i^{t h}$ such labeled neuron. A recurrent neural network allows cycles in the edges, which means that data from later processing stages are fed back to earlier stages [Wikipedia, 2008b].

\subsubsection{Special Case: Feed Forward Neural Network}

This thesis deals with a special case of the general neural network in Fig. 2.8 on page 30, which is the feed forward neural network Fig. 2.9 on page 32. This is a special case because: $i$ ) neurons are organized into groups (called layers), which does not have to be the case in the general neural network, $i i)$ neurons get excited. In the classical case, a neuron is excited (firing) if the activation function associated to that neuron is true. Another definition is that the activation function is above a threshold value or that the activation function is a bounded function, such as the logistic function. This thesis is different from the classical case because no activation function is explicitly defined to bound the neuron outputs nor, for that matter, is a threshold value defined. Therefore, so as long as a neuron receives input and produces 
output, then the neuron is considered to be in an excited state. iii) neuron excitement is not bounded as in the classical case because the equations that can be extracted from a neural network will then not internally contain such bounding functions. iv) neurons cannot excite themselves (i.e. no loops or cycles), which does not have to be the case in the general neural network, v) neurons can only excite neurons in their adjacent group, which is the feed forward aspect of the feed forward neural network (i.e. layer $i$ neurons can only excite layer $i+1$ neurons), although other possibilities exist such as when layer $i$ neurons can excite neurons in all layers greater than $i$, and vi) a neuron can decide which (of the possibly many) input neurons may have permission to excite it, which is different from the classical case in which weights determine to which degree a neuron in the previous layer is able to excite the current neuron.

In Fig. 2.9 on page 32 the input vector $\left\langle x_{1}, x_{2}, \cdots, x_{n}\right\rangle_{i}$ are the arguments to each of the neurons (more precisely, functions) within the first layer $\left(\ell_{1}\right)$. To be more clear, the input vector is a row in the input data matrix that contains the set of variables (columns). In general, the inputs to the neurons in the $i^{\text {th }}$ layer $\left(\ell_{i}\right)$ are the outputs from the neurons in the $(i-1)^{\text {st }}$ layer $\left(\ell_{i-1}\right)$. The output layer in Fig. 2.9 on page 32 is $\ell_{3}$ and is the output vector for the whole network. Such an output vector $<o_{1}^{\ell_{\text {out }}}, o_{2}^{\ell_{\text {out }}}, \cdots, o_{c}^{\ell_{\text {out }}}>_{i}$ will have different dimensions depending on whether there are multiple output layer neurons or only one output layer neuron. Point (iv) essentially relaxes the feed forward neural network architecture compared to classical approaches in such a way that only an upper bound is what needs to be specified; this means that not all edges will be used in Fig. 2.9 on page 32 at all times during the evolutionary process of learning the neuron functions.

A general extension to GEP for vector valued functions was previously introduced [Valdés et al., 2007], whereby GEP individuals consist of multiple chromosomes. Such an extension was the starting point for the construction of a technique to evolve feed forward neural networks. 


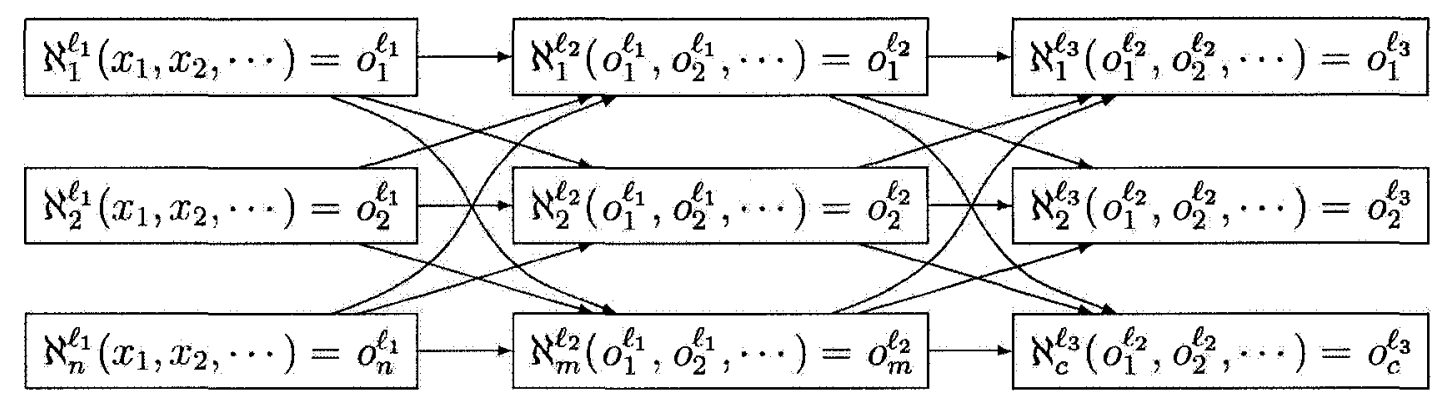

Figure 2.9: Special case of the general neural network: a feed forward neural network (FFNN) for one specific architecture containing 3 layers and $(n+m+c)$ neurons $(N N: n-m-c)$ where neurons in layer $i$ are connected to neurons in layer $i+$ 1. Possibilities exist providing more code reuse (e.g. neurons in layer $i$ could be connected to neurons in all layers greater than $i$ ). All activity (e.g. activation, aggregation, weight learning, bias, etc) occurs in a neuron. Neurons are not required to use all inputs; so only an upper bound on the network connectivity is specified. (See Fig. 4.3 on page 66 )

\subsubsection{Type of Neural Network used in this Thesis}

This thesis uses a layered feed forward neural network with either 1 neuron in the output layer or multiple neurons in the output layer. In the latter case, the number of neurons is equal to the number of classes within the data. For this network, layer $i$ will have connections to neurons in layer $i+1$. Other types of feedforward networks exist. For example, layer $i$ may have connections to neurons in all layers greater than $i$, allowing neurons to use neurons as many times as there are layers within the neural network. In addition, other types of networks exist, as was demonstrated, for example, in Fig. 2.8 on page 30.

\subsubsection{First Model of a Neuron}

Once the Neuron Doctrine [Fodstad, 2001] was established (i.e. an agreement, in biological terms, of what a neuron entailed) a systematic study (as a Logical Calculus using a two-valued propositional logic) could be made with the following physical assumptions [McCulloch and Pitts, 1943]:

1. The activity of a neuron is an "all-or-none" process.

2. A certain fixed number of synapses must be excited within the period of latent 
addition in order to excite a neuron at any time, and this number is independent of previous activity and position on the neuron.

3. The only significant delay within the nervous system is synaptic delay.

4. The activity of any inhibitory synapse absolutely prevents excitation of the neuron at that time.

5. The structure of the net does not change with time.

The first mathematical model of a neuron is shown in Eq. 2.11 on page 33 and was introduced by [McCulloch and Pitts, 1943]. The weighted sum of input signals is compared to a threshold to determine whether or not the neuron fires; has output 1 or 0. When discussing a set of neurons, such as in a neural network in Section 2.2.5.5 on page 33, then we need to discuss more than one neuron, which is indicated in Eq. 2.11 on page 33 by the subscript on $o_{i}$. This was the first attempt to describe what the brain does and, in addition, it showed that simple neural networks could compute any arithmetic or logical function ${ }^{13}$.

$$
o_{i}=\left\{\begin{array}{l}
1 \text { if } \sum_{j=1}^{\text {inDegree }\left(\aleph_{i}\right)} w_{j} \cdot x_{j} \geq T \\
0 \text { otherwise }
\end{array}\right.
$$

\subsubsection{Perceptron}

The biological and psychological insights [McCulloch and Pitts, 1943] led to the construction of the first Artificial Neural Network [Rosenblatt, 1958], [Rosenblatt, 1961], [Block, 1962], based on probability theory rather then symbolic logic. The following three fundamental questions were posed in [Rosenblatt, 1958] if an understanding of perceptual recognition, generalization, recall and thinking were to be attempted:

1. How is information about the physical world sensed, or detected, by the biological system?

\footnotetext{
${ }^{13}$ Hence, the science of mind became a science of signals based on binary logic with clearly defined units of perception and precise rules of formation and transformation for representing mental states, which was aimed at bridging the gulf between body and mind (matter and form) [Kay, 2001].
} 
2. In what form is information stored, or remembered?

3. How does information contained in storage, or in memory, influence recognition and behavior?

That work was derived from basic physical variables ${ }^{14}$. The perceptron and its variants (e.g. [Gallant, 1990]) provide the only known effective general solution to the problem of building a regression function from examples in the high-dimensional input / high-dimensional output case [Hecht-Nielsen, 2004].

In Rosenblatt's perceptron, the inputs are binary and no bias was included. It was based on the McCulloch-Pitts model of the neuron with the threshold activation function. In Eq. 2.12 on page 34 Rosenblatt normally [Widrow and Lehr, 1990] set $\alpha$ to 1 .

$$
\overleftarrow{W}_{k+1}=\overleftarrow{W}_{k}+\alpha \frac{\varepsilon}{2} \bar{X}_{k}
$$

where $\varepsilon$ was defined to be the difference between the desired response and the actual output. A proof related to convergence of the perceptron [Novikoff, 1962] is outside the thesis scope of this thesis. There are other models for a neuron.

\subsubsection{Training via Classical Backpropagation}

In 1969, Minsky and Papert showed that perceptrons are incapable of learning concepts that are linearly inseparable (See Fig. 2.10 on page 35). This problem was overcome with the backpropagation algorithm. There are many variants [Werbos, 1990], [Cun, 1988], [Poggio and Girosi, 1990]. The simplest form is [Widrow and Lehr, 1990]:

... backpropagation training begins by presenting an input pattern vector $\bar{X}$ to the network, sweeping forward through the system to generate an output response vector $\bar{Y}$, and computing the errors at each output. The next step involves sweeping the effects of the errors backward through the network to associate a "squared error derivative" $\delta$, and finally updating

\footnotetext{
${ }^{14}$ According to a remark by Kistiakowsky, "given seven parameters, I could fit an elephant" does not hold in this case because it is claimed [Rosenblatt, 1958, p.407] that the parameters can be measured independently of the predicted behaviour.
} 

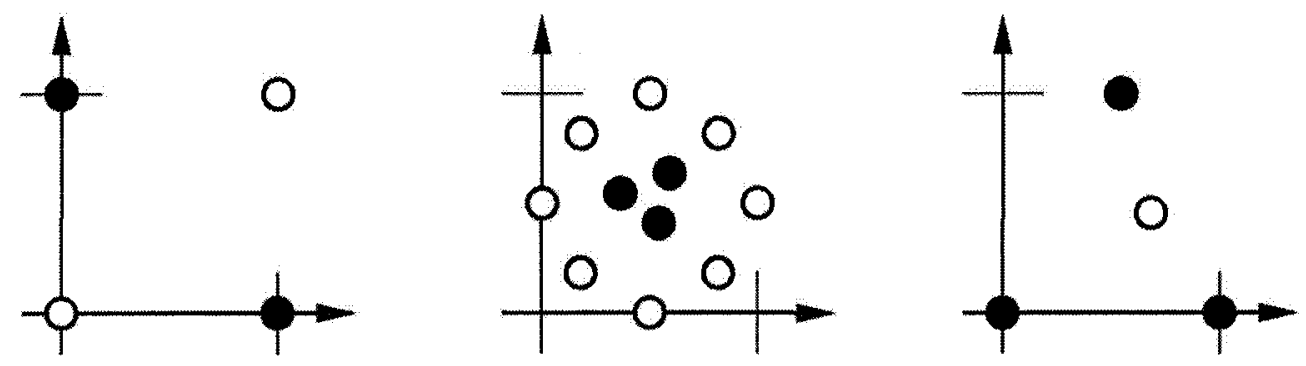

Figure 2.10: Examples of Linearly Inseparable Problems that caused a decline in Neural Network research in the 1970s [Hagan et al., 2002].

the weights of each Adeline ${ }^{15}$ based upon the corresponding gradient. A new pattern is then presented and the process is repeated. The initial weight values are normally set to small random numbers. The algorithm will not work properly with multilayer networks if the initial weights are either zero or poorly chosen nonzero values ${ }^{16}$.

A proof that any continuous mapping can be approximately realized by RumelhartHinton-Williams' multilayer neural networks with at least one hidden layer whose output functions are sigmoid functions [Funahashi, 1989] is outside the thesis scope.

The method of steepest descent can be described by the relation:

$$
\overleftarrow{W}_{k+1}=\overleftarrow{W}_{k}+\mu\left(-\nabla_{k}\right)
$$

where $\mu$ is a parameter that controls stability and rate of convergence, and $\nabla_{k}$ is the value of the gradient at a point on the Mean-Squared-Error (MSE) surface corresponding to $\overleftarrow{W}=\overleftarrow{W}_{k}$ [Widrow and Lehr, 1990].

\subsection{Other Background Information}

In addition to the foundation algorithms, GAs, GPs and GEPs, the concepts of Rough Sets, including reducts and minimum reducts, an Individual Dichotomization Algorithm and brief notes about relevant statistics are described.

\footnotetext{
${ }^{15}$ neuron

${ }^{16}$ [Widrow and Lehr, 1990, p.1433] references work stating that there is experimental evidence suggesting that it is advisable to choose the initial weights of each hidden layer to ensure that only a few neurons will be saturated.
} 


\subsubsection{Rough Sets Theory}

The Rough Set Theory [Pawlak, 1982], [Pawlak, 1991] works on the assumption that in order to define a set, some knowledge about the elements is needed. This is in contrast to the classical approach where a set is uniquely defined by its elements. In Rough Sets Theory, some elements may be indiscernible from the point of view of the available information and it turns out that vagueness and uncertainty are strongly related to indiscernibility.

\subsubsection{Reducts and Minimum Reducts}

Let $O=\left\{o_{1}, o_{2}, \cdots, o_{m}\right\}$ be a set of $m$ objects and $A=\left\{a_{1}, a_{2}, \cdots, a_{N}\right\}$ a set of $N$ attributes. Let $d$ be a special attribute called the decision attribute. $O$ is consistent if $\forall k, n, \forall i \in[1, N], a_{i}\left(o_{k}\right)=a_{i}\left(o_{n}\right) \rightarrow d\left(o_{k}\right)=d\left(o_{n}\right)$. A reduct is a subset $R \subseteq A$ so that $\forall k, n, \forall a \in R, a\left(o_{k}\right)=a\left(o_{n}\right) \rightarrow d\left(o_{k}\right)=d\left(o_{n}\right)$. Minimal reducts are those for which no proper subset is a reduct and are extremely important, as decision rules can be constructed from them [Bazan et al., 1994]. However, the problem of reduct computation is NP-hard, and several heuristics have been proposed [Wróblewski, 2001].

\subsubsection{Reduct Computation}

Genetic algorithms are the most popular representative of the evolutionary computation family of algorithms [Bäck et al., 1997], [Bäck et al., 2000]. They have been used as an approach to reduct computation by [Wróblewski, 1995], which proposed several methods based on the notion of a distinction table; which is a $\left(m^{2}-m\right) / 2 \times(N+1)$ matrix $B$ where columns $i$ are attributes (the last one is the decision attribute $d$ ) and the rows are pairs of objects $k, n$. For every row $i \in[1, N]$ and every $k, n \in[1, m]$ the values of $\mathrm{B}$ are constructed as follows: $B[(k, n), i]=1$ if $a_{i}\left(o_{k}\right) \neq a_{i}\left(o_{n}\right)$ and 0 otherwise. For the last row $B[(k, n), N+1]=1$ if $d\left(o_{k}\right)=d\left(o_{n}\right)$ and 0 otherwise. In terms of $B$, a reduct is a subset of columns $R$ with the property [Wróblewski, 1995] $\forall k, n, \exists i \in R,(B[(k, n), i]=1) \vee(B[(k, n), N+1]=1)$. In its simplest representation, a GA with binary chromosomes of length $N$ encodes subsets of attributes (the indices of the chromosomes for which the value is 1). The evolution is guided by a fitness 
function given by: $F(r)=\left(\left(N-L_{r}\right) / N\right)+C_{r} / K$, where $r$ is a chromosome, $L_{r}$ is the cardinality of the set of attributes (given by the number of $1 \mathrm{~s}$ in the chromosome, $C_{r}$ is the number of object pairs (with different values of the decision attribute) which are discerned by the attributes in $R . K=(m(m-1)) / 2$ is the no. of object pairs.

\subsubsection{Individual Dichotomization Algorithm}

This is a simple screening algorithm used with the purpose of finding individual attributes that are relevant from the point of view of their ability to differentiate the classes (in a binary problem), when their values are dichotomized. The inputs for the algorithm are: i) the values of a given attribute $A$ for all the objects, ii) the classes $c_{1}, c_{2}$ associated with each sample, and iii) a probability threshold $p_{T}$. The algorithm proceeds as follows: (1) construction of the set of distinct values of $\mathrm{A}$ (call it $\Delta$ ). If $O$ is the set of objects and $A(o)$ is the value of the attribute for any object $o \in O, \Delta=\left\{\delta_{1}, \delta_{2}, \cdots, \delta_{k}\right\},(k \in[1, \operatorname{card}(O)])$ with the following properties: $\left(\forall \delta_{i}, \delta_{j} \in \Delta, \delta_{i} \neq \delta_{j}\right),(\forall o \in O, \exists \delta \in \Delta$ s.t. $A(o)=\delta)$ and $(\forall \delta \in \Delta, \exists o \in O$ s.t. $A(o)=\delta$ ). (2) sort $\Delta$ in increasing order. (3) construct the set $\hat{\Delta}$ composed by the mean of all consecutive values of $\Delta$. That is, for every pair $\delta_{i}, \delta_{i+1} \in \Delta$ compute $\left(\hat{\delta}_{i}=\left(\delta_{i}+\delta_{i+1}\right) / 2\right.$. Clearly, $\hat{\Delta}$ has one element less than $\Delta$. (4) use each ( $\hat{\delta}_{i} \in \hat{\Delta}$ as a binary threshold for the values of attribute $A$. This divides the set of objects into two disjointed classes $A_{1}, A_{2}$. (5) compute the contingency table of $A_{1}, A_{2}$ vs $c_{1}, c_{2}$ (6) on the table, compute the conditional probabilities $p_{1}=p\left(c_{1} / A_{1}\right)$, $p_{2}=p\left(c_{1} / A_{2}\right)$ and retain $p_{\max }=\max \left(p_{1}, p_{2}\right)$. (7) if $p_{\max } \geq p_{T}$ select the attribute as relevant, and discard it otherwise. The process is repeated for all attributes and the resulting set of selected attributes gives an indication about how many of them contain a differentiation power equal or better than the pre-set probability threshold $p_{T}$. Specifically, if $p_{T}=1$ the algorithm will give a set of attributes such that each of them (individually) will perfectly differentiate the classes $\left\{c_{1}, c_{2}\right\}$.

\subsubsection{Statistics}

The word "parameter" in the modern statistical sense was introduced in 1921 by Ronald A. Fisher [Stigler, 2005]. This notion is used when attempting to understand 
what a statistical model entails [McCullagh, 2002]. Both discussions are outside the scope of this thesis. However, the following concepts are relevant:

Regression For example, [Pearson, 1901], given a set of $x, y$ pairs of data find a "best-fitting" straight line. Analytically take $y=a_{0}+a_{1} x$ where $y, x$ are the observed variables; find the "best" values for the constants $a_{0}, a_{1}$. The variable $y$ is able to be treated as dependant and $x$ as independent (or vice versa). In other words, the most probable value of $y$ for a given value of $x$ is not necessarily given by the same relation as the most probable value of $x$ for a given value of $y$. Fig. 2.11 on page 39 shows an example where "best-fit" means the sum of the squares of the perpendiculars from the system of points upon the line. Regression is used within this thesis because a neural network is able to perform nonlinear regression. For example, see ${ }_{f_{2}} \aleph_{>1}^{\ell_{\text {out }}}$.

Komolgorov-Smirnov Non-Parametric Statistic [Press et al., 2002, p.625]: The significance level of an observed value of $D$ (as a disproof of the null hypothesis $\left(H_{0}\right)$ that the distributions are the same). Small values of the probability show that the cumulative distribution function of the first data set is significantly different from that of the second data set.

\subsubsection{Visualization of High Dimensional Spaces}

There are many possible paradigms for creating visual spaces within data mining, which is used within this thesis in Section 7.2 on page 134. In particular Virtual Reality (VR) is a suitable paradigm for several reasons. It is flexible: allows the choice of different ways to represent the objects according to differences in human perception. VR allows immersion: the user can navigate inside the data and interact with the objects in the world. It creates a living experience: the user is a passive observer, but an actor in the world. VR is broad and deep: the user may see the VR world as a whole, or concentrate on details. Also very important is that its use does not require any special background knowledge. A virtual reality technique for visual data mining on heterogeneous, imprecise and incomplete information systems was introduced in [Valdés, 2002], [Valdés, 2003] (see also http://www.hybridstrategies.com). It is 


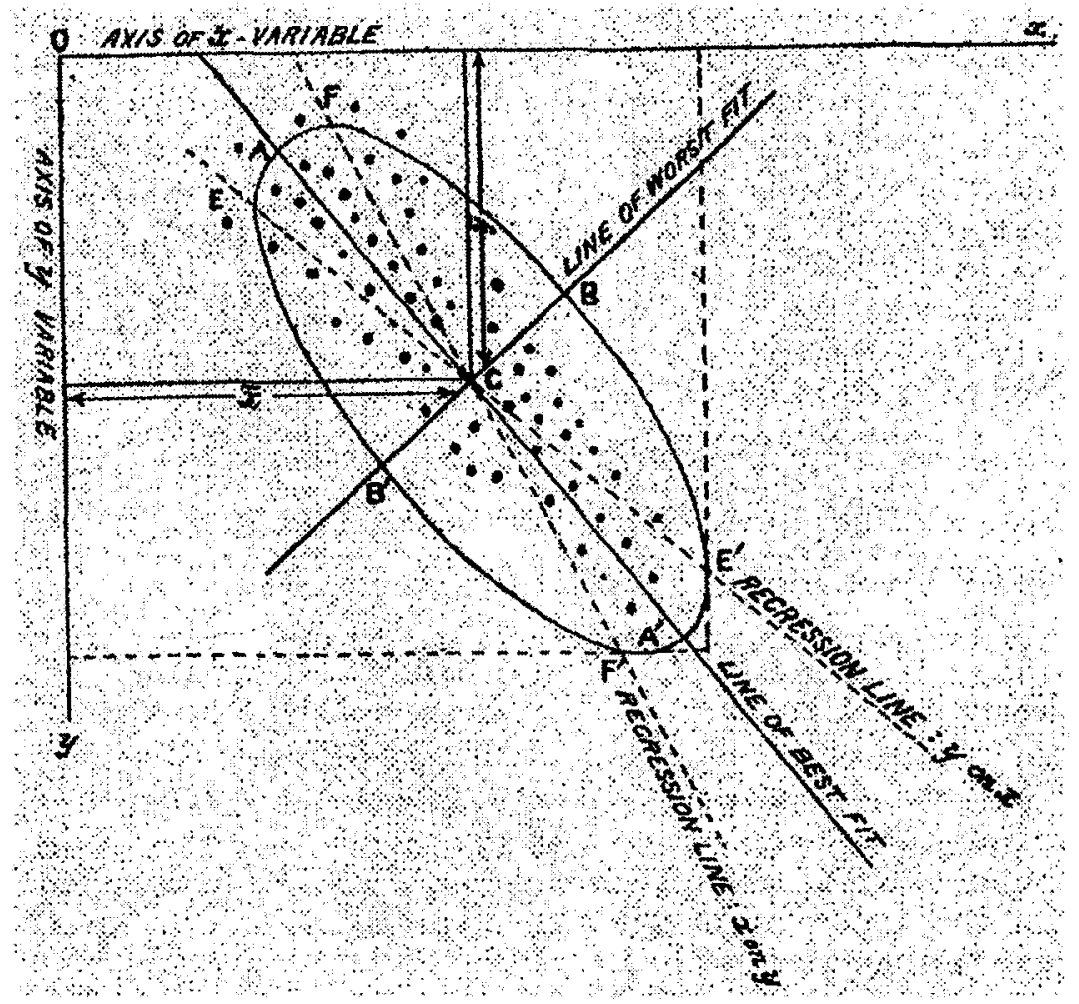

Figure 2.11: A figure from [Pearson, 1901] explaining regression, in which, for example, he states that: "The best-fitting straight line for a system of points in a space of any order goes through the centroid of the system". $\overline{E E^{\prime}}$ is $y=\frac{r_{x y} \sigma_{y}}{\sigma x} x$ and $\overline{F F^{\prime}}$ is $x=\frac{r_{x y} \sigma_{x}}{\sigma y} y$ 
oriented to the understanding of large heterogeneous, incomplete and imprecise data, as well as symbolic knowledge. The notion of data is not restricted to databases, but includes logical relations and other forms of both structured and non-structured knowledge.

One of the steps in the construction of a VR space for data representation is the transformation of the original set of attributes describing the objects under study, often defining a heterogeneous high dimensional space, into another space of small dimension (typically 2-3) with intuitive metric (e.g. Euclidean). The operation usually involves a non-linear transformation; implying some information loss. There are basically two kinds of spaces sought: $i$ ) spaces preserving the structure of the objects as determined by the original set of attributes, and $i$ i) spaces preserving the distribution of an existing class defined over the set of objects.

Different information sources are associated with the attributes, relations and functions. They are described by the so called source sets $\left(\Psi_{i}\right)$, constructed according to the nature of the information to represent. Source sets also account for inconplete information. A heterogeneous domain [Valdés, 2003] is a Cartesian product of a collection of source sets: $\hat{\mathcal{H}}^{n}=\Psi_{1} \times \cdots \times \Psi_{n}$, where $n>0$ is the number of information sources to consider. For example, in a domain where objects are described by continuous crisp quantities, discrete features, fuzzy features, time-series, images, and graphs, they can be represented as Cartesian products of subsets of real numbers $(\hat{R})$, nominal $(\hat{N})$ or ordinal sets $(\hat{O})$, fuzzy sets $(\hat{F})$, sets of images $(\hat{I})$, sets of time series $(\hat{S})$ and sets of graphs $(\hat{G})$, respectively (all extended for allow missing values). The heterogeneous domain is $\hat{\mathcal{H}}^{n}=\hat{N}^{n_{N}} \times \hat{O}^{n_{O}} \times \hat{R}^{n_{R}} \times \hat{F}^{n_{F}} \times \hat{I}^{n_{I}} \times \hat{S}^{n_{S}} \times \hat{G}^{n_{G}}$, where $n_{N}$ is the number of nominal sets, $n_{O}$ of ordinal sets, $n_{R}$ of real-valued sets , $n_{F}$ of fuzzy sets, $n_{I}$ of image-valued sets, $n_{S}$ of time-series sets, and $n_{G}$ of graph-valued sets, respectively $\left(n=n_{N}+n_{O}+n_{R}+n_{F}+n_{I}+n_{S}+n_{G}\right)$.

A virtual reality space is the tuple $\Upsilon=<\underline{O}, G, B, \Re^{m}, g_{o}, l, g_{r}, b, r>$, where $\underline{O}$ is a relational structure $\left(\underline{Q}=<O, \Gamma^{v}>, O\right.$ is a finite set of objects, and $\Gamma^{v}$ is a set of relations); $G$ is a non-empty set of geometries representing the different objects and relations; $B$ is a non-empty set of behaviors of the objects in the virtual world; $\Re^{m} \subset \mathbb{R}^{m}$ is a metric space of dimension $m$ (euclidean or not) which will be the 
actual virtual reality geometric space. The other elements are mappings: $g_{0}: O \rightarrow G$, $\varphi: O \rightarrow \Re^{m}, g_{r}: \Gamma^{v} \rightarrow G, b: O \rightarrow B$.

Data structure is one of the most important elements to consider and this is the case when the location and adjacency relationships between the objects $O$ in $\Upsilon$ should give an indication of the similarity relationships [Chandon and Pinson, 1981], [Borg and Lingoes, 1987] between the objects in $\hat{\mathcal{H}}^{n}$ (in the classical cases studied in MOO $\hat{\mathcal{H}}^{n}=\Re^{n}$ ), as given by the set of original attributes [Valdés, 2003]. $\varphi$ can be constructed to maximize some metric/non-metric structure preservation criteria as has been done for decades in multidimensional scaling. [Kruskal, 1964], [Borg and Lingoes, 1987], or to minimize some error measure of information loss [Sammon, 1969]. If $\delta_{i j}$ is a dissimilarity measure between any two objects $i, j(i, j \in$ $[1, N]$, where $N$ is the number of objects), and $\zeta_{i^{v} j^{v}}$ is another dissimilarity measure defined on objects $i^{v}, j^{v} \in O$ from $\Upsilon\left(i^{v}=\phi(i), j^{v}=\phi(j)\right)$. Examples of error measures frequently used are [Kruskal, 1964], [Sammon, 1969] and [Borg and Lingoes, 1987]:

$$
\begin{aligned}
\text { S stress } & =\sqrt{\frac{\sum_{i<j}\left(\delta_{i j}^{2}-\zeta_{i j}^{2}\right)^{2}}{\sum_{i<j} \delta_{i j}^{4}}}, \\
\text { Sammon error } & =\frac{1}{\sum_{i<j} \delta_{i j}} \frac{\sum_{i<j}\left(\delta_{i j}-\zeta_{i j}\right)^{2}}{\delta_{i j}} \\
\text { Quadratic Loss } & =\sum_{i<j}\left(\delta_{i j}-\zeta_{i j}\right)^{2}
\end{aligned}
$$

Classical algorithms have been used for directly optimizing these measures, like Steepest descent, conjugate gradient Fletcher-Reeves, Powell, Levenberg-Marquardt, and others. The number of different similarity, dissimilarity and distance functions definable for the different kinds of source sets is immense. Moreover, similarities and distances can be transformed into dissimilarities according to a wide variety of schemes, thus providing a rich framework.

Froin the point of view of the property(s) which the objects in the space must satisfy, several paradigms can be considered for building a transformed space for constructing visual representations [Valdés and Barton, 2006]:

Unsupervised: The location of the objects in the space should preserve some 
structural property of the data, dependent only on the set of descriptor attributes. Any class information is ignored. The space sought should have minimal distortion.

Supervised: The goal is to produce a space where the objects are maximally discriminated w.r.t. a class distribution. The preservation of any structural property of the data is ignored, and the space can be distorted as much as required in order to maximize class discrimination.

Mixed: A space compromising the two goals is sought. Some amount of distortion is allowed in order to exhibit class differentiation and the object distribution should retain in a degree the structural property defined by the descriptor attributes. Very often these two goals are conflicting.

From the point of view of their mathematical nature, the mappings can be:

Implicit: the images of the transformed objects are computed directly and the algorithm does not provide a function representation.

Explicit: the function performing the mapping is found by the procedure and the images of the objects are obtained by applying the function. Two sub-types are:

- analytical functions: for example, as an algebraic representation constructed via genetic programming.

- general function approximators: for example, as neural networks, fuzzy systems, or others.

An explicit $\varphi$ is useful for both practical and theoretical reasons. On one hand, in dynamic data sets (e.g. systems being monitored or incremental data bases) an explicit transform $\varphi$ will speed up the update of the VR irnformation system. On another hand, it can give semantics to the attributes of the VR space, thus acting as a general dimensionality reducer. 


\subsection{Chapter Summary}

The main background material has been presented within this chapter. Some brief initial considerations were discussed with respect to the data, the algorithm, the search methodology, the internal representation and the external representation (results). A general evolutionary computation specification was made and the Genetic Algorithm, Genetic Programming Algorithm, and Gene Expression Programming Algorithm were described. In addition, the neural network algorithm was was explained, including one variant of the backpropagation algorithm. Other topics were also covered, including, Rough Sets Theory, the Individual Dichotomization Algorithm and the statistical topics of regression and non-parametric statistics. Now that these preliminary concepts have been discussed, it is possible to investigate the literature in order to understand what lines of research are currently being pursued. 


\section{Chapter 3}

\section{Related Work: State of the Art}

The previous chapter provided specifications and references for the relevant algorithms along with some biological notions. The particular variant of GP, the Gene Expression Programming Algorithm in Section 2.2.4 on page 22, was selected for investigation within this thesis. It is now important to critique the state of the art research in which a description of the perceived deficiencies need to be reported and which ones this thesis is attempting to address. The concept of neural network and the different types are discussed in Section 2.2.5.1 on page 29 .

All variants of genetic programming may potentially be used to solve symbolic regression problems. The issue is how a particular variant performs. This thesis proposes to learn neuron functions through the use of one particular variant of GP, namely, an extended version of GEP [Valdés et al., 2007]. The extension was proposed in order to solve the problem of computing vector valued functions by extending the concept of an individual to consist of multiple chromosomes. Other approaches exist; for example, through the notion of a multicellular system, where an individual is composed of multiple cells and each cell has one chromosome [Ferreira, 2006a].

\subsection{Classical Neural Networks}

An investigation of the spontaneous emergence of new computational capabilities from the collective behavior of large numbers of simple processing elements was performed in [Hopfield, 1982]. Those equivalent components are neurons.

A theorem showing that three-layered perceptrons with an infinite number of computing units can represent arbitrary mapping if the desired mapping and the input-output characteristics of the computing units satisfy some constraints is reported [Irie and Miyake, 1988]. They state that two-layered perceptrons (simple perceptrons) cannot represent arbitrary functions but that three-layers prove necessary 
and sufficient. This thesis does not create classical neurons with activation functions, but, never-the-less, three layers are used and, empirically, the non-linear decomposability problem is solved. In other words, NN-GEP is able to produce a potentially non-linear analytic formula for separating the classes.

A procedure for determining the optimal topology of a static three-layer neural network has been described in [Wang et al., 1994]. They report a practical procedure to implement their method and it is applied to both simulated and real process data.

Misconceptions about Neural Networks when they are used as approximators have been described in [Carpenter and Barthelemy, 1994].

Work has been performed in which the sigmoid activation function was replaced with an exponential function with other activations discussed [Specht, 1990]. That work uses a 4 layer network and their training regime was 200,000 times faster than back-propagation (See Section 2.2.5.6 on page 34).

Three deterministic techniques (variable metric, conjugate gradient, and steepest descent), and a stochastic technique are investigated to train a neural network [Barnard, 1992]. It is found that the stochastic technique is preferable on problems with large training sets and that the convergence rates of the variable metric and conjugate gradient techniques are similar.

The number of iterations can be reduced using backpropagation training if after each iteration step the weights are changed by a simple mutation as it is done in GAs [Kinnebrock, 1994]. In the examples described the computing time was reduced to $5.5 \%$ of backpropagation time in the average; 120,000 average number of iterations down to 1,400$)$.

Using classical weight- and activation-based neural networks does not always produce good results. Some practical limitations are reported [Burden, 1994]. For example, the architecture of the network, the activation function for each neuron and the learning rate must all be chosen. They report that for spectral data, the derivative of the logistic function, when used as an activation function, can improve the ANN performance by a factor of 2 to 3 . They also compare a polynomial with the equivalent number of coefficients as the network has weights. They state that in some cases, the network may be inefficient in its use of weights. They conclude by stating that, 
among other things, in the case where the function to be fitted is representable by known analytic functions then the ANN can be made more efficient by choosing an appropriate activation function for the neurons. However, in such cases, the ANN is unlikely to compete with a direct fitting procedure using either the relevant analytic function or a spline method, which can achieve a very close fit though at the expense of using a set of at least three polynomial coefficients for every data point.

Neural network have been labeled a "black box" because they provide little explanatory insight into the relative influence of the independent variables in the prediction process. A number of methods for understanding the mechanics (e.g. Neural Interpretation Diagram, Garson's algorithm, sensitivity analysis) are proposed for classical networks in [Olden and Jackson, 2002]. They also propose ways of removing connections. This thesis aims to also remove the "black box" label of neural networks.

Work to improve the backpropagation algorithm has been done; in particular, two new variants are proposed in [Wan et al., 2009]. This thesis is not attempting to improve any particular backpropagation algorithm, but rather, is attempting to provide an alternative learning algorithm using evolutionary computation.

\subsubsection{Comparisons}

A comparison of GA and backpropagation for training neural networks has been reported in [Sexton and Gupta, 2000]. Using chaotic time series functions, they empirically compared a genetic algorithm with backpropagation for training ANNs. They report that in every problem instance, the GA algorithm was found to provide statistically superior solutions in less CPU time. They also state that further research is needed to investigate the sensitivity of the various GA parameters.

A comparison of GA and simulated annealing, two global search techniques, for optimizing neural networks has been reported in [Sexton et al., 1999]. They state that optimizing means to closely approximate unknown functions to any degree of desired accuracy. They stated that there are no rules for adjusting the configuration of backpropagation or simulated annealing in order to produce better results, but that heuristics do exist in the literature. They found that the ANN solutions dominated those of SA, which dominated those of backpropagation, in every case for interpolation 
and extrapolation.

\subsubsection{Nonlinear discriminant analysis (NDA)}

Minimizing the error at the output of the network is equivalent to maximizing a particular norm, the network discriminant function, at the output of the hidden units [Webb and Lowe, 1990]. The first part of the network is explicitly performing a nonlinear transformation of the data into a space in which the classes can be more easily separated. Other work on NDA is performed in [Mao and Jain, 1993] and [Mao and Jain, 1995]. This thesis performs NDA, for example, in Section 7.4.2 on page 146 (See Fig. 7.14 on page 147).

\subsubsection{Some Alternatives to Classical Networks}

Training nonconvergent networks is of interest in certain applications [de Garis, 1990], [de Garis, 1991] but is not applicable for this thesis. It is interesting that the same author also proposes the concept of Neurite Networks [de Garis, 1993] in which neural networks grow.

The general regression neural network (GRNN) is a one-pass learning algorithm [Specht, 1991]. The concept of regression was described in Section 2.3.3 on page 37 and for neural networks means that the network output vector is allowed to be continuous. Put another way, the network is not trying to learn discrete values, such as classes, but is trying to fit itself to a continuous range of values, such as height of a person. Section 2.2.5.1 on page 29 discusses a general neural network.

Searches over a family of polynomial functions in which a linear function is examined first, followed by increasingly higher order [Carpenter and Barthelemy, 1994]. They claim that their approach may yield more insight into the nature of the function being approximated than with neural network approximations.

Reasoning Neural Networks (RN) are layered feedforward networks (See Section 2.2.5.2 on page 30) with weights and structural change [Tsaih, 1998]. Section 3.4.2 on page 54 discusses ways in which the structure (i.e. network architecture) can be improved. 
Alternative neural circuit design is proposed [Maass, 2000] and theoretically analysed. Namely, feedforward networks (See Section 2.2.5.2 on page 30) with threshold or sigmoidal activation functions (See Section 4.2.5 on page 66) and with positive and negative weights can be replaced by a single soft winner-take-all gate (neuron) applied to positive weighted sums (the only nonlinear operation). The problem with this approach, is, again, that the weights need to be learned. This thesis has a function $\left({ }_{f_{1}} \aleph_{>1}^{\ell_{\text {out }}}\right)$ that, essentially, performs winner-take-all, and, in addition, sums of weighted input variables are not the only possibility.

The Neulonet is a weight and activation based neural network [Tan and Chia, 2001]. It describes a genetic programming approach that constructs neural logic networks from training data. This thesis also uses genetic programming, but does not require activation functions within the set of primitive functions that are used by GP. That is, this thesis provides a more flexible approach, since the activation function, aggregation function, and the bias and weights are completely learned within the neuron.

Learning polynomial feedforward neural networks (PFNNs) involves identification of the network architecture, that is, polynomial terms, and finding the weights, that is, polynomial term coefficients. An approach for learning PFNNs is reported in [N.Y.Nikolaev and Iba, 2003]. First, the polynomial network structure is found using genetic programming. Second, further adjustment of the best discovered network weights by an especially derived backpropagation algorithm for higher order networks with polynomial activation functions is performed.

A polynomial neural network has been discussed in [Misra et al., 2008]. The idea is for small order polynomials to be used within the neurons. This thesis does not restrict the order of the polynomials within the neurons, nor does it restrict the neurons to only contain polynomials.

\subsection{Evolution of a Neural Network is Necessary}

Work has been done [Bullinaria, 2005] to investigate the possibility of speeding up learning of classically trained networks by learning from how the evolutionary training process proceeds. They conclude that 4 independent learning rates are an improvement over the classical 1 learning rate for the whole network. They also state that the 
complex interactions of the evolved parameters indicate that the evolutionary process cannot be dispensed. Therefore, they state that evolution really is worth the effort and that the evolutionary process can be used to find robust parameter configurations. This is the objective of the work resulting from this thesis (See Section 7.2 on page 134).

\subsection{Training a Network}

Literature for training a classical feed forward neural network (FFNN) was reported in Section 3.1 on page 44. The current section reports literature that applies more generally to training a FFNN. For example, work has been done to train a FFNN with Genetic Algorithms (GAs) [Montana and Davis, 1989]; [Yao, 1993] provides a review.

\subsubsection{Undertraining}

Underdetermined neural networks are networks in which the number of training pairs used to train the network are fewer than the number of weights and biases associated with the network [Carpenter and Barthelemy, 1994]. Such networks can be trained to exactly duplicate the exact function at the design points. However, just as with underdetermined polynomial approximations, they are not unique. Such networks, starting from different initial values of the weights and biases, when trained will, in general, yield different approximations. A necessary condition for obtaining a unique approximation is to have the number of design points used to train the approximation equal to or greater than the number of parameters associated with the approximation. This thesis does not explicitly use weights, but there is certainly a possibility that solutions can be found that fit the data very well, but do not fit interpolated or extrapolated points. The undertraining problem is related to algorithm termination criteria (See Section $\mathbf{4 . 5}$ on page 76 ). 


\subsubsection{Overtraining}

In supervised learning, overtraining is always a potential problem [Rowland, 2003]. They review ways to validate predictive models in order to avoid overtraining; including: leave $k$ out, partitioning into training, testing and validation data, and cross-validation. This thesis uses the partitioning approach, and divides the original input data into training and testing sets. The overtraining problem is related to algorithm termination criteria (See Section 4.5 on page 76).

\subsubsection{Input Data is Large}

When a large data source is used to train a neural network model, considerable effort and time are required to obtain reliable outcomes. Work has been done to reduce the size the that data and it was found that models built using this reduced data set show nearly identical performance on the same set of test cases than models built using the full size data set [Colmenares and Perez, 1998]. They compare stratified and random construction. This thesis uses the random approach in order to select training and testing data from the original data sets (See Table 6.5 on page 102). This thesis does not perform experiments on data sets that have millions of objects or millions of attributes.

\subsubsection{Input Data is Small}

The selection of a feedback neural network model that best represents a particular process based on limited available data is performed in [Schenker and Agarwal, 1996], Of the 3 approaches they investigate, they claim that only the cross-validation approach accurately selects the best model and reliably assesses its quality. This thesis uses the random approach in order to select training and testing data from the original data sets (See Table 6.5 on page 102) due to software limitations for performing cross-validation. 


\subsubsection{Input Data Contains Missing Points}

If the training data contains missing values within the input or attributes have more uncertainty than others, then [Tresp et al., 1994] proposes how to train the network. Their work also hints that substituting missing values by their respective attribute means can be harmful. This is because if, at the substituted point, the difference between network prediction and target is large, then that point contributes significantly to the gradient. But it is very unlikely that the substituted value was the true input. This thesis completely removes objects that contain missing values (See, for example, Section 6.1.2 on page 94).

\subsubsection{Presentation Order}

Work has been done to use a GA to select the presentation order of training patterns in order to improve the performance of a simplified fuzzy ARTMAP classification [Palaniappan and Eswaran, 2009]. Within this thesis, presentation order does not have an effect.

\subsubsection{Interpreting A Neural Network (extracting rules)}

The ability to interpret a neural network's structure is a desirable feature. Work has been done to learn indirectly encoded networks with arbitrary connectivity using a context-free grammar guided GP search [Tsakonas et al., 2004]. Their contribution is to extract rules. This thesis does not try to extract rules from networks, but rather, to extract analytic functions (See Chapter 7 on page 109).

\subsubsection{Finding More than one Network at a Time}

A collection of neural networks is called an ensemble of neural networks. The goal of this thesis is to find one neural network.

A GA is used to train and/or prune a neural network [Benediktsson et al., 1997]. They investigate combining multiple classifiers based on statistical consensus theory rather than leuristic or ad hoc combination schemes.

A study of the issue of diversity in ensembles of neural networks is performed in 
[Brown, 2004]. The one neural network case is not the focus of their work. However, in this thesis it is the focus because one network is a universal function approximator [Hornik, 1989].

A fixed size collection of neural networks are each individually trained and then coinbined into an ensemble using genetic programming [Johansson et al., 2006]. The authors claim that the use of genetic programming makes it possible to not only consider ensembles of different sizes, but also to use ensembles as intermediate building blocks. The final result is therefore more correctly described as an ensemble of neural network ensembles. This thesis constructs 1 neural network (not an ensemble) and uses less than 50 individuals in the population (See Table 6.6 on page 104), which is significantly smaller than [Johansson et al., 2006], who uses 10 neural networks and 1,000 individuals.

The multi-class problem was addressed in [Zhao, 2008] where an ensemble of networks is used, with each solving a 2 class sub-problem. This thesis only uses 1 network, not an ensemble. The network output mapping function plays a large role in addressing the multi-class problem (See Section 4.2 .5 on page 66 ).

\subsection{Improving Classical Backpropagation}

Section 3.1 on page 44 reported literature for classical backpropagation based training for FFNNs, while Section $\mathbf{3 . 3}$ on page 49 reported general issues related to training a FFNN. This current section discusses the issues involved when evolutionary computation is used to improve the classical approach.

The earliest description of the tree-like representation and operators for use in

the application of Genetic Algorithms to computer programs was in [Cramer, 1985]. The first work that was reported to optimization of the weights and neural network architecture using genetic programming was first proposed in [Koza and Rice, 1991]. A review of evolutionary neural networks is provided in [Yao, 1993] that discusses the evolution of i) weights, $i i$ ) architectures, and $i i i)$ learning rules. 


\subsubsection{Improving the Biases and Weights}

A genetic algorithm is used to learn the weights and biases for a feedforward network [Montana and Davis, 1989]. It reports the improvements gained over backpropagation. It also investigates the addition of more and more domain-specific knowledge.

A learning algorithm for neural networks based on genetic algorithms is proposed in which a general asymmetric network is learned [Bornholdt and Graudenz, 1992]. Activation functions are used within that work.

Keeping neural networks simpler by minimizing the description length of the weights and suggesting schemes for encoding the weights has been performed in [Hinton and van Camp, 1993]. They are motivated by the fact that practical learning tasks sometimes contain very little training data; potentially causing an overly complicated model to be learned. This thesis does not explicitly use weights. However, the encoding of the constants within GEP may benefit from [Hinton and van Camp, 1993]'s work, in the sense that potentially fewer constants may be needed.

The weights for the neurons within a layer are encoded within a chromosome in [Chow and Chu, 1994]. Hence, there is one chromosome per layer. This thesis has one chromosome per neuron and learns the complete function for the neuron, whereas that work learns the weights.

Linearly separable and nonseparable classes are used to train a network in which a nonlinear selection for creating mating pools and a weighted error as a fitness function is used in [Pal and Bhandari, 1994].

A genetic algorithm for training a feedforward neural network (See Section 2.2.5.2 on page 30) using an adjacency matrix was proposed in [Siddique and Tokhi, 2001] and compared with a backpropagation algorithm.

Bayesian neural network trained using Markov Chain Monte Carlo (MCMC) and genetic programming in binary space within Metropolis framework is proposed in [Marwala, 2007]. This approach learns the weights.

Many studies have mapped a bit-string genotype using a genetic algorithm to represent network architectures to improve performance of backpropagation networks (BPN). [Huang et al., 2009] propose how to re-evaluate the weight matrices of BPN during evolution. 


\subsubsection{Improving the Architecture}

The algorithm proposed in this thesis does not learn the architecture of a neural network in the sense of learning the number of neurons per layer or the number of layers ${ }^{1}$. This thesis does learn the connections between neurons. This is a true statement because a terminal symbol in a neuron may or may not be used and such a terminal symbol reflects the output of a neuron in a previous layer (or an input variable). Therefore, this thesis learns the neural network connectivity within a fixed number of neurons and a fixed number of layers.

A neurogenetic learning algorithm to design and train neural networks using a GA and a gradient-descend approach for fine-tuning of the network weights and biases is proposed in [Kitano, 1994]. That work extends previous work from 1990.

The construction of neural networks using a dual representation, a linear chromosome and a graph, in which the evolution of the architecture and the weights are performed, is reported in [Pujol and Poli, 1998].

The structure and weights for an ANN is learned by hybridizing GP and decision trees in [Matsumoto and Tazaki, 1998]. The learning rule of the decision tree is found by GP. The fitness values are further improved by using a hybrid GP technique which is combined GP and backpropagation.

A hybrid method of modular neural networks and genetic programming is reported in [Cho and Shimohara, 1998]. Weights are used within their work.

Constraints on the search space or restructuring the architecture of the neural network are unnecessary if a sufficiently complex initial architecture and an appropriate global search algorithm is used [Sexton et al., 1998]. Further work is presented in [Gupta and Sexton, 1999].

Special terminals and functions are introduced into GP by [Gros, 2003] in order to learn the complete architecture of a neural network. That work claims that layered networks have limited expressiveness and that layered networks are hard to build with genetic programming. That work uses a threshold activation function with a threshold of 1 . This thesis does not introduce any special terminals nor does it introduce any special functions. This thesis has a fixed number of neurons, but the connectivity

\footnotetext{
${ }^{1}$ The number of neurons per layer is fixed and heuristically chosen within this thesis.
} 
is free to evolve. This thesis does not use explicit activation functions as is done within that work. It is hard to know why it is claimed that layered networks have limited expressiveness, especially since it has been claimed that layered networks are universal approximators [Hornik, 1989].

GAs can learn the network architecture [Alsultanny and Aqel, 2003] and it is also claimed that GPs are capable [Ritchie et al., 2003]. The weights and the number of hidden nodes can be adjusted [Tsaih, 2003]. There is even work with GEP to learn the network weights, thresholds and architecture [Ferreira, 2004]. All of these works learn within the classical formulation of a neural network, that is that weights and activation functions must be used. It is the main claim of this thesis, that this usage does not need to occur.

A method that guides the selection of both input variables and a sparse connectivity of the lower layer of connections in feedforward neural networks of multi-layer perceptron type with one layer of hidden nonlinear units and a single linear output node is presented in [Saxén and Pettersson, 2006]. This thesis also selects input variables and does not require full network connectivity.

\subsubsection{Improving the Learning Rules}

The learning method is critical for obtaining good generalization in neural networks with limited training data. The classical backpropagation training algorithm suffers from several problems such as sensitivity to the initial conditions and very slow convergence [Radi and Poli, 1999]. Work has been done in [Bengio et al., 1994] that used Genetic programming to find the values of the learning rule parameters, the optimal number of parameters and the form of the learning rule. Then, [Radi and Poli, 1999] has done further work to discover efficient learning rules for the hidden and output layers. They state that the best rule found with genetic programming outperformed the well-known backpropagation algorithm for a given set of tasks. These approaches all lie within the classical approach in which neurons have activation functions and weights are used. This thesis does not restrict the neurons in this manner. 


\subsubsection{Improving the Activation Functions}

Both one-hidden-layer and two-hidden-layer network architectures were explored by GA in [Ferentinos, 2005]. Each gene also encoded the type of activation functions in both hidden and output nodes of the network and the type of minimization algorithm that was used by the backpropagation algorithm for the training of the network.

Setting free parameters in the neuron's activation function, in other words, parameters of the activation function, is performed in [Kahramanli and Allahverdi, 2009].

Results are reported for a single-layer perceptron with a Choquet fuzzy integralbased neuron rather than the sigmoid function [Hu and Tsai, 2009]. They state their approach is comparable to the traditional single-layer perceptron and to other fuzzy classification methods. This thesis doesn't use activation functions because more general functions are used.

\subsubsection{Neuroevolution}

Neuroevolution is defined [Stanley, 2004] to be the artificial evolution of neural networks, which is a method for finding the right topology and connection weights for a particular problem. Difficult tasks might require complex networks with many connections, all of which must have the appropriate weights. Even if a network exists that can solve the task, evolution may not be able to find it in such a high-dimensional search space. The solution presented in [Stanley, 2004], is called NeuroEvolution of Augmenting Topologies (NEAT) and uses complexification. NEAT begins by searching in a space of simple networks, and gradually makes them more complex as the search progresses. By starting minimally, NEAT has been shown to find efficient and robust solutions when compared to neuroevolution methods that begin with large fixed or randomized topologies; by elaborating on existing solutions, it can gradually construct even highly complex solutions.

\subsection{Network Encodings}

A very clear paper summarizing strategies for encoding neural networks has been reported [Koehn, 1996]. 
The permutation problem is the fact that the same network can be represented in a genetic coding by many different codifications [García-Pedrajas et al,, 2006]. To overcome the problem, they report a new crossover operator. Within this thesis, it is agreed that, two codings of networks might lead to the same resulting analytic function. For example, two networks will be equivalent if two neurons in a selected layer are swapped and then the terminals in the next layer are renamed in order to reflect the swap. This problem is an interesting avenue for future research (See Eq. 7.2 on page 115 and Eq. $\mathbf{7 . 3}$ on page 115 ).

\subsubsection{Direct Encoding}

An empirical comparison of four encoding methods for evolving neural networks was made in [Grönroos, 1999], with the details given in his thesis. It is unclear exactly what is performed, but they state that each neuron and connection is encoded separately in the genome. In addition, they state that the network topology is encoded and that the connection weights are trained with a separate neural learning algorithm. What is interesting, is that the performance of the trained networks are used as fitness values to guide the evolution of the population, as is done within this thesis. They conclude that Kitano's graph generation grammar performs best for the 3 classification problems investigated. Kitano's method is such that connections are determined from a connection matrix that is generated from a $1 \times 1$ symbol matrix using genetically encoded rewriting rules that replace each element with a $2 \times 2$ matrix. This thesis does not explicitly learn a connection matrix, but rather, lets the selection of terminals by GEP within a neuron (chromosome) be the definition for learning connections.

The Gene Expression Programming's linear chromosome (See Section 2.2.4.1 on page 23) was modified so that a single neural network could be encoded within it [Ferreira, 2004]; including the architecture, the weights and thresholds and provides an automatic design of neural networks. However, it requires a special set of genetic operators and also requires one to specify the set of allowable functions at the neurons. This thesis does not explicitly require weights since everything is learned within the neuron. This means that no activation function needs to be specified. 


\subsubsection{Indirect Encoding}

According to [dos Santos Caldas de Matos et al., 2007] Cellular encoding as proposed by Frederic Gruau's PhD in 1994 has a proven track record for evolving neural networks for a wide range of problems. The encoding specifies a set of graph-rewriting instructions that are evolved directly. The original model, only evolved simple threshold networks: the neurons were threshold neurons with thresholds of either 0 or 1 , with the connections between the neurons being either -1 or 1 . In this thesis, neurons do not have thresholds.

An investigation of Topology and Weight Evolving Artificial Neural Network (TWEANN) methods which evolve both topology and parameters (weights) of neural networks and is based on indirect encoding was performed in [Drchal and Snorek, 2008]. They investigated known tree-based indirect encodings: Cellular Encoding and Edge Encoding. They used Gene Expression Programming (GEP) and Grammatical Evolution (GE) to optimize the trees. They conclude that on a benchmark problem, GE was able to find a solution about 7 times faster then GEP but that GEP solutions were more compact. This thesis considers simpler analytic functions with respect to number of input variable to be of greater benefit than solution time for computation (See Section 7.1.1.1 on page 115, Section 7.1.2.1 on page 121 or Section 7.1.3.1 on page 128). Therefore, if [Drchal and Snorek, 2008] has claimed that GEP produces more compact solutions, then this might indicate that GEP will produce simpler analytic functions over GE when extracted from a neural network as is done within this thesis. Of course, compactness and simplicity are two different issues and further research is required to more thoroughly compare GEP and GE for learning neural networks.

\subsubsection{Common Encoding for Direct and Indirect Encodings}

A Common Genetic Encoding for networks that can be applied to both direct and indirect encoding methods has been developed [Kassahun et al., 2007]. The encoding has the following properties: i) completeness (i.e. it should be able to represent all types of valid phenotype networks), ii) closed (i.e. every valid genotype represents a valid phenotype and the encoding should be closed under genetic operators), and 
iii) flexibility (i.e. can apply the encoding for direct or indirect encoding of neural networks). This thesis does not encode the neural network within the genome and, in addition, the Gene Expression Programming's Karva language (See Section 2.2.4.1 on page 23) ensures that the encoding is complete and closed. This thesis does not need to propose any new genetic operators in order to encode a neural network.

\subsection{Classification}

A simple example of a classification problem is given in Section 4.1 on page 62 . This section briefly discusses work that others have done in reporting appropriate classification error measures and for addressing the multiple class $(>2)$ problem.

\subsubsection{Classification Error Measures}

An experimental analysis of a large number of possible classification error measures has been performed in [Ferri et al., 2009]. The error measures used within this thesis are those within the fitness function for an individual and are reported in Section 4.3.1 on page 73 .

\subsubsection{Multiple Class Problem}

Work has been done to design classifiers for the c-class ( $c>=2$ ) problem using GP [Muni et al., 2004]. They propose a modified crossover operator, a new nutation operator and a new concept of unfitness of a tree to select trees for genetic operators; in order for unfit trees to become fit. They proposed OR-ing chromosomes in the terminal population in order to get a better performing classifier. They perform conflict resolution ${ }^{2}$ using weights and heuristics, and the resulting classifier may report "don't know". This thesis does not need to modify GP's operators in order to learn a neural network classifier. In addition, this thesis reports "unclassifiable" whenever a prediction lies directly on a decision boundary, which is a highly improbable situation. This thesis uses two approaches for addressing the multiple class problem; namely,

\footnotetext{
${ }^{2}$ Classification conflicts are when more than one class is predicted. A conflict resolution is a policy for deciding what to do to when this occurs. For example, one may report "don't know", or one may randomly pick one of the predicted classes to report. The author of this thesis learned in 1999 from Dr. Goodenough that "unclassifiable" is a more appropriate term.
} 
the 2 neural network output mapping functions ${ }_{f_{1}} \aleph_{>1}^{\ell_{\text {out }}}$ and ${ }_{f_{2}} \aleph_{>1}^{\ell_{\text {out }}}$ (See Section 4.2.5 on page 66).

An ensemble of neural networks might also be used to address this problem (See Section 3.3.8 on page 51).

\subsection{Hybrid Approaches}

Work has been done to construct feedforward or recurrent networks with all arbitrary number of hidden levels using grammatical evolution for classification and regression problems [Tsoulos et al., 2005].

Work has been done to develop a Flexible Neural tree using GP in which the parameters are optimized by a memetic algorithm (MA) [Chen et al., 2006]. They investigate a set of activation functions.

A hybrid GA and back-propagation algorithm is proposed in order to classify garment defect types [Yuen et al., 2009].

\subsection{Genetic Programming Variants}

There are many variants of genetic programming. Only Gene Expression Programming is investigated within this thesis. This section gives examples for several genetic programming variants, but does not attempt to fully explain nor to fully compare Gene Expression Programming to the particular GP variant; that is a future research direction.

Symbolic regression by combining a Genetic Algorithm and a Genetic Programming Algorithm has been performed in [Howard and D'Angelo, 1995].

Parallel Distributed Genetic Programming is a form of GP in which symbolic and neural processing elements can be combined [Poli, 1996] [Poli, 1997]. This work represents a neural network within a fixed sized grid and uses special operators in order to construct valid expressions (networks). The networks are allowed to grow as large as the grid. Layers are grid rows. Neural networks with and without weights are learned but classical activation functions are used. 
A new form of linear genetic programming [Brameier and Banzhaf, 2001] was introduced and compared with classical neural networks. Its main characteristic in comparison to tree-based GP is that expressions of a functional programming language (like LISP) are substituted by programs of an imperative language (like C). They use a population size of 5,000 .

Incremental Evaluation with Tree Adjoining Grammar Guided Genetic Programming is proposed in [Tuan-Hao et al., 2006] and compared to standard Genetic Programming and the original Tree Adjoining Grammar Guided Genetic Programming.

Multiobjective genetic programming is used within [Krawiec, 2007] in which a set of non-dominated solutions are sought that are as close as possible to the true Pareto Front. In most real world applications, the Pareto Front is not known (See, for example, [Valdés and Barton, 2006] [Valdés and Barton, 2007]).

An automatically defined function (ADF) is a function that is dynamically evolved during a run of genetic programming and that might be called by a calling program that is concurrently being evolved [Koza et al., 1996]. See Fig. 4.1 on page 64 and Fig. 4.2 on page 65 in Section 4.2 .1 on page 64 .

\subsection{Chapter Summary}

The literature related to evolving neural networks was surveyed within this chapter. There are many interesting research avenues, but those that stood out are: i) NEAT's use of complexification [Stanley, 2004] ii) the encoding of weights when little training data exists [Hinton and van Camp, 1993], which might be applicable to constants within NN-GEP, iii) the encoding of weights for all neurons within one layer within one chromosome [Chow and Chu, 1994], iv) the description of underdetermined networks [Carpenter and Barthelemy, 1994], which leads to the idea that it might be interesting to add additional test points that are interpolations or extrapolations of training examples, and v) a multicellular system, where an individual is composed of multiple cells and each cell has one chromosome [Ferreira, 2006a]. In conclusion, most approaches take the classical neural network and use weights and activation functions; this thesis does not. The next chapter describes the theory of what will be investigated. 


\section{Chapter 4}

\section{NN-GEP Theory}

... applied mathematics can generate phantom physical questions...

[Maddy, 2008]

This chapter focuses the discussion from the previous chapter that presented all of the directly related work performed by others to the current chapter in which related concepts and ideas that are needed within this thesis are reported. This chapter provides descriptions of the four major steps when using a genetic algorithm [Koza, 1992, p.27] and the overlapping five major steps for using a genetic programming algorithm [Koza, 1994]. In particular, this chapter will present: i) a general representation scheme for how a classification or non-linear discrimination problem can be solved; including the particular approach using feed forward neural networks, ii) a discussion of the terminals and primitive functions used within the general representation scheme, iii) the general way in which the fitness measure in conceived, iv) the ways in which the algorithm can be controlled, and $v$ ) what criteria are used for stopping execution of the optimization process.

\subsection{A Simple Example}

Imagine that a fleet of cars belongs to a company and some of the cars work and some of them do not. These imaginary cars have 5 sensors on them that are able to measure various things about the state of the car. Hopefully, these measurements are somehow related to the fact that a car works or not. But, of course, we do not know why a car is not working and we did not create the sensors or even decide what they are measuring. Our problem is to try and find out which of the measurements (if any) are related and be able to explain to the company why a car is or is not working. Perhaps this explanation can even be used to predict the behaviour of a new car that is added to the fleet. 
With these 5 sensors and a class (working or not working) we would like to build a classifier. The choice of classifier that we want to build depends on our goals. Do we only want something that performs very well at informing us of when the imaginary car is working? Or do we want to understand the inner details of the classifier? Let us suppose we choose the latter. There are different types of classifier. Let us suppose we want the classifier to be representable as an equation. Again, we have many choices. This thesis investigates a neural network classifier that can be represented as an equation once we have finished learning.

At this point, we have the 5 measurements to use along with supervised information (the class). How do we learn such a neural network classifier? The details are within this section. In brief, an evolutionary algorithm uses sets of potential solutions and, over time, combines and modifies them in order to search for a good solution that can be reported as the final result of the algorithm. An example result is an equation such as:

$$
\text { IF } 22.36+0.024 \cdot V_{5}-12.64 \cdot V_{1}\left\{\begin{aligned}
\geq 69.37 & \Rightarrow c_{1} \text { (i.e. Working) } \\
<69.37 & \Rightarrow c_{2} \text { (i.e. Not Working) }
\end{aligned}\right.
$$

which indicates that 2 sensors $\left(V_{1}\right.$ and $V_{5}$ ) are potentially important, and that if those sensor outputs are combined in a certain way, then an indication of whether a car is working or not may be conjectured.

\subsection{Representation Scheme for a Problem}

The problem is construction of a classifier or a non-linear discriminant function for particular training and testing data sets. The representation is a neural network. Section 2.2.5.1 on page 29 described the general neural network and the specific case of a feedforward neural network that is used within this thesis is described in Section 2.2.5.2 on page 30. The feedforward neural network will have its neurons subjected to evolutionary computation. That is, the functions associated with a neuron will be learned. No activation functions will be applied to the individual neurons, which is the classical approach (See Section 2.2.5.6 on page 34). The neural network's architecture is specified as a fixed number of neurons and a fixed number of layers. The connections are free to be learned within those bounds. 


\subsubsection{Special Case: FFNN as Automatically Defined Functions}

Koza (e.g. [Koza, 1995a]) proposed the idea of reusing subexpressions by combining them within a main program (See Section 3.8 on page 60). The subexpressions are called Automatically Defined Functions (ADFs). Ferreira also has an approach that reuses subexpressions [Ferreira, 2006a] the difference being that the main program is not as rigid as Koza's because it is also allowed to evolve Fig. 4.1 on page 64 . This thesis proposes learning functions for neurons within a neural network architecture. A special case of this work is that of learning using a special form of ADFs because subexpressions may be reused. Fig. 4.2 on page 65 shows a very particular case of a FFNN with 2 layers and connections from layer $i$ to layer $i+1$. Neurons in the first layer may be considered to be variants of ADFs in the sense that code is being reused, while neurons in the second layer may be considered to be the set of main programs as opposed to the classical case of 1 rigid main program.
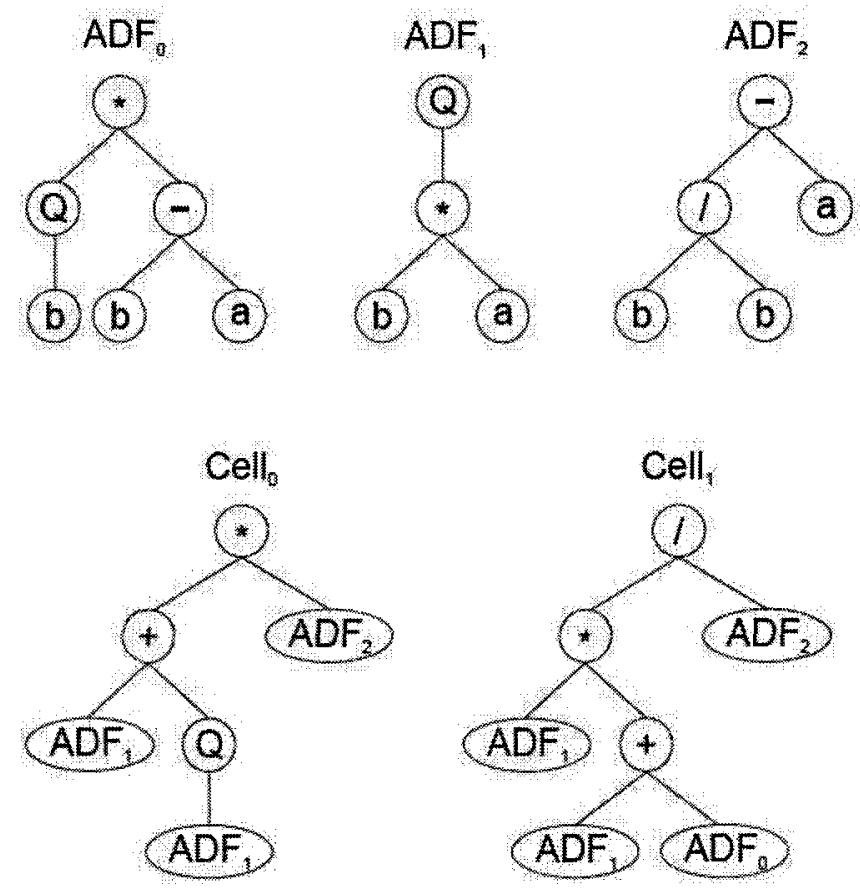

Figure 4.1: Example of a multicellular system [Ferreira, 2006a] with three Automatically Defined Functions and two different main programs expressed in different cells. The cells combine the ADFs in different ways. 


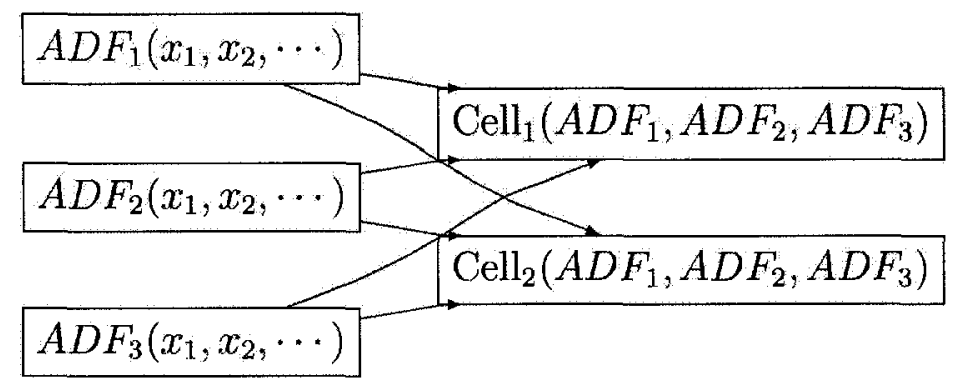

Figure 4.2: Special case of the general neural network: a feed forward neural network (FFNN) interpreted as learning with Automatically Defined Functions (ADFs). The $N N: 3-2$ architecture can be used to learn 3 ADFs for 2 problems simultaneously. Other approaches exist for learning ADFs [Koza, 1995a] [Ferreira, 2006a].

\subsubsection{Special Case: FFNN for Nonlinear Discriminant Analysis}

If the output layer in a feed forward network is not considered, then the remaining network may be used for non-linear discriminant analysis. In this thesis, for the Magnetic Resonance Spectra results, the penultimate layer has $m=1$ neuron Fig. 7.14 on page 147 .

\subsubsection{Special Case: FFNN with Multiple Output Layer Neurons}

The neural network output vector $\left\langle o_{1}^{\ell_{\text {out }}}, o_{2}^{\ell_{\text {out }}}, \cdots, o_{c}^{\ell_{\text {out }}}>_{i}\right.$ for object $i$ will have dimension based on the number of classes $(c)$ in the data when the architecture of the neural network is specified to contain multiple neurons in that layer (which is a design decision). Fig. 2.9 on page 32 gives an example of a multiple output layer neural network with $n$ variables in the data, 1 hidden layer with $m$ neurons, and an output vector $<o_{1}^{\ell_{\text {out }}}, o_{2}^{\ell_{\text {out }}}, \cdots, o_{c}^{\ell_{\text {out }}}>_{i}$ for the $i^{\text {th }}$ object of dimension $c$.

\subsubsection{Special Case: FFNN with Single Output Layer Neuron}

The neural network output vector $\left\langle o_{1}^{\ell_{\text {out }}}, o_{2}^{\ell_{\text {out }}}, \cdots, o_{c}^{\ell_{\text {out }}}\right\rangle_{i}$ for object $i$ is not constrained to contain precisely the same dimension as the number of classes $(c)$ in the data. In fact, there may be exactly one neuron in the output layer (Fig. 4.3 on page 66) instead of $c$ neurons (Fig. 2.9 on page 32). Section 4.2 .5 on page 66 discusses the output vector. 


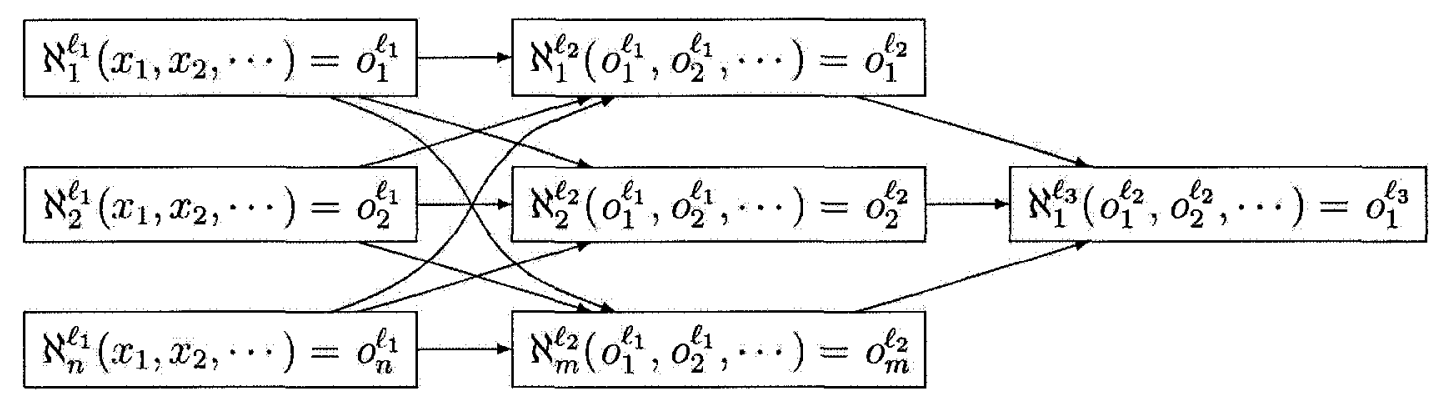

Figure 4.3: Special case of the general neural network: a feed forward neural network (FFNN) for one specific architecture containing 3 layers and $(n+m+1)$ neurons $(N N: n-m-1)$. (See Fig. 2.9 on page 32 )

\subsubsection{Neural Network Output Vector}

If a neural network is being constructed in order to perform classification, as is the case in this thesis, then the $i^{\text {th }}$ object $\left\langle x_{1}, x_{2}, \cdots, x_{n}\right\rangle_{i}$ will produce (after being pushed through the network) an associated degree of membership to each class. In other words, after pushing the training example through the network an output vector $<o_{1}^{\ell_{\text {out }}}, o_{2}^{\ell_{\text {out }}}, \cdots, o_{c}^{\ell_{\text {out }}}>_{i}$ will be constructed. Therefore, it can be seen that the choice of neural network architecture directly affects the dimension of the output vector. Two cases are considered within this theses. The first is when the number of output neurons is equal to the number of classes contained within the data and the second case is when only a single output neuron is used in the output layer.

\subsubsection{Output Vector: Number of Output Neurons Equal to Number of Classes}

The choice of neural network architecture determines the number of neurons in the output layer. In the classical case, the feed forward neural network output vector $<o_{1}^{\ell_{\text {out }}}, o_{2}^{\ell_{\text {out }}}, \cdots, o_{c}^{\ell_{\text {out }}}>_{i}$ is of dimension equal to the number of classes $(c)$ in the data. The output vector may be interpreted as containing fuzzy class memberships. That is, each last layer neuron output value may represent a degree of class membership rather than merely a crisp value in which a discrete class value is reported. In this classical case, multiple neurons will potentially be excited, so a decision needs to be made as to which class the network is considering object $i$ to belong. 


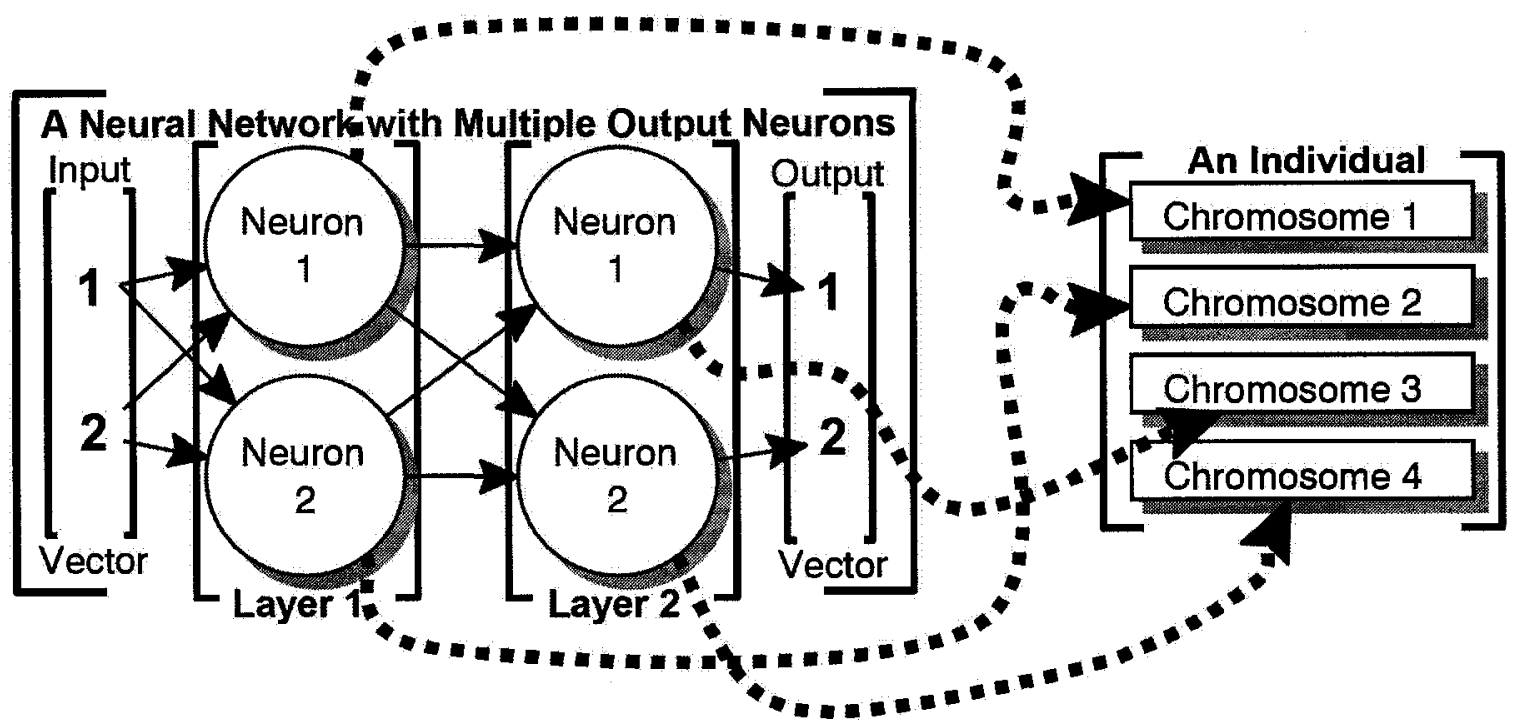

Figure 4.4: How a feed forward neural network is represented within an individual containing multiple chromosomes. Each neuron is associated to a chromosome. Each chromosome contains a subset of the permitted terminals and primitive functions. The chromosomes are learned within the specific EC algorithm, in this case GEP.

This thesis investigates two functions for making such a decision. The first function $f_{1} \aleph_{>1}^{\ell_{\text {out }}}$ makes a decision as to object class membership based on the most excited neuron firing. That is, if neuron $k$ has the largest floating point output when considering all of the $c$ neurons in the output layer, then the class prediction for the input object is class $k$. There is a very small, non-zero probability that two or more output neurons may have equal floating point values. If such a situation happens, then "unclassifiable" is reported.

The second investigated function $f_{2} \aleph_{>1}^{\ell}$ out makes a decision as to object class membership based on the "closest" output vector for the neural network to a target binary vector $\delta_{i}^{\aleph}$ for class $i$ within a set of $c$ binary vectors for all of the possible expected classes. Each binary vector $\delta_{k}^{\aleph}$ has length equal to the number of classes c. Each binary vector $\delta_{k}^{\aleph}$ contains only one set bit in location $k$; all other bits are unset. Therefore, the set will contain $c$ binary vectors, with all pairwise binary vector intersections being empty. The "closest" binary vector(s) will be used to determine the neural network class prediction(s). If exactly one class is predicted, then that class will be considered to be the neural network class prediction. If more than one class is predicted, then no classes will be predicted and the neural network output 
will be "unclassifiable". A class will be predicted if the neural network output vector is close enough to the associated binary vector for that class. Those output vectors that are less than $\varepsilon^{1}$ (See Table 6.6 on page 104) away from the target binary vector are considered to be close enough. This is a form of residual analysis because the residual (difference) between a logistically mapped output and the expected output is performed, albeit within an threshold of tolerance.

Both functions $\left({ }_{f_{1}} \aleph_{>1}^{\ell_{\text {out }}}\right.$ and $\left.{ }_{f_{2}} \aleph_{>1}^{\ell_{\text {out }}}\right)$ may yield predictions for more than one class for a particular input object. In such a situation, a class prediction conflict resolution must be chosen. One example is randomly picking one of the classes, which would make the fitness function, and hence results, non-deterministic. The class prediction resolution policy chosen in this thesis is to report the prediction as "unclassifiable" in order to keep determinism.

\subsubsection{Output Vector: Single Output Neuron}

Classically, the number of output neurons is equal to the number of classes in the data. However, an alternative approach is to have one output neuron. This may be a good idea because it implies a reduction of $c-1$ chromosomes in order to represent a neural network. A more compact representation potentially implies lower computational overhead because of the reduction in the number of chromosomes. However, the representation may be such that the evolutionary computation algorithm, in this case GEP, will require more generations to find an acceptable solution. Experimentation on a variety of data sets may indicate what is the general trend.

When the neural network output vector $\left\langle o_{1}^{\ell_{\text {out }}}, o_{2}^{\ell_{\text {out }}}, \cdots, o_{c}^{\ell_{\text {out }}}>_{i}\right.$ only contains one value because only one output neuron exists in the network architecture, then a mapping function must be chosen. The identity function cannot be used (i.e. the raw neuron output) because the output would have to be precisely 1 or precisely 2 up to the number of classes $c$ (i.e. discrete values). All other raw neuron outputs would need to be dealt with in some manner. In addition, the raw neuron output may be a very large or very small number. How should a class be assigned? That is the crux of the matter.

\footnotetext{
${ }^{1} \varepsilon=0.001$ within this thesis
} 


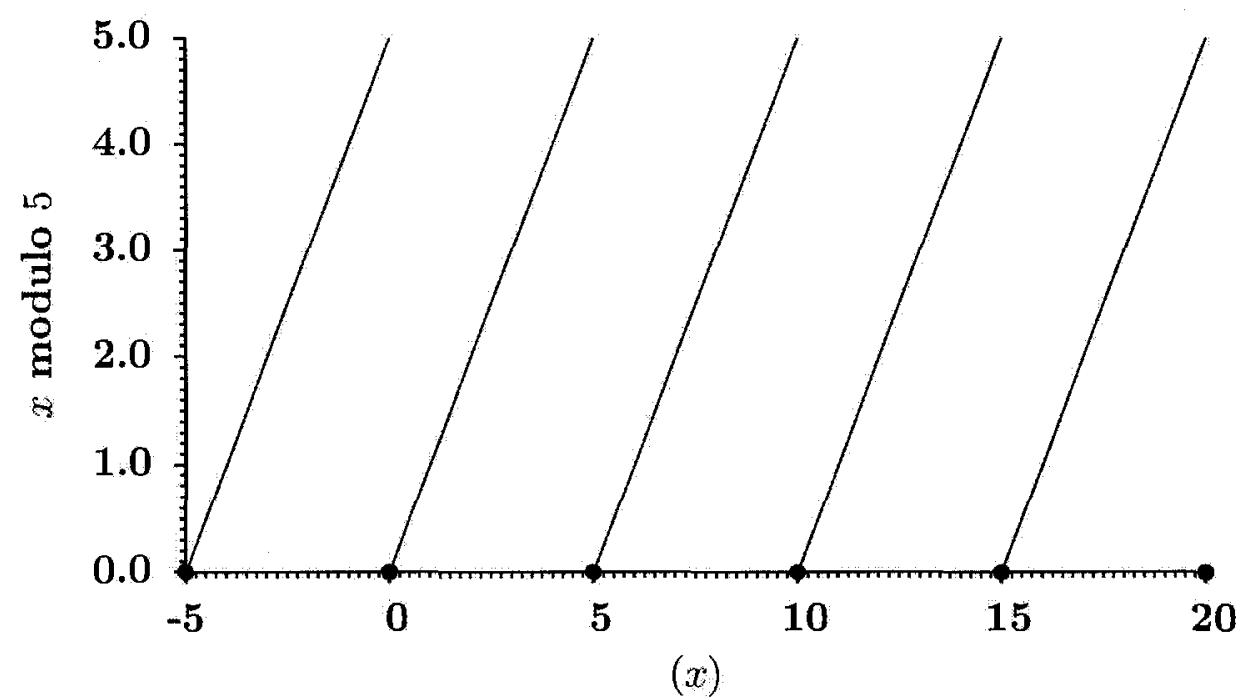

Figure 4.5: Example of the Euclidean definition of the modulo operation [Boute, 1992]. This may or may not be the implementation within a particular programming language.

The modulo operator is a first potential choice ${ }^{2}$ when deciding upon a mapping function $(f: R \rightarrow[0 . . c])$ in order to map the last (single) output neuron into an interval based on the number of classes $(c)$ in the data. However, $i$ ) there are discontinuities within the modulo operator as can be seen in Fig. $\mathbf{4 . 5}$ on page 69 , ii) the Euclidean definition of the modulo operation [Boute, 1992] is not necessarily what a particular programming language implements, and $i i i)$ the modulo operator becomes a function when a choice is made with respect to the end points of the line segments (which side of the segment to include/exclude).

Examples of classical activation functions are not directly usable. For example, Fig. 4.6 on page 70 shows two classical activation functions, where the logistic function has a range [0..1] and the atan function has a range [-1..1]. It can be seen that the latter (i.e. atan) more slowly converges to its asymptotes than the former (i.e.

\footnotetext{
${ }^{2}$ Another choice would be an operator that performs rounding or truncation. The question becomes "To what should -15.3123 be rounded?" If the answer is -15 then to what class should the object be assigned? The first class? A reject class? It is easier if all of $\mathbb{R}$ is used, and then this issue is avoided.
} 


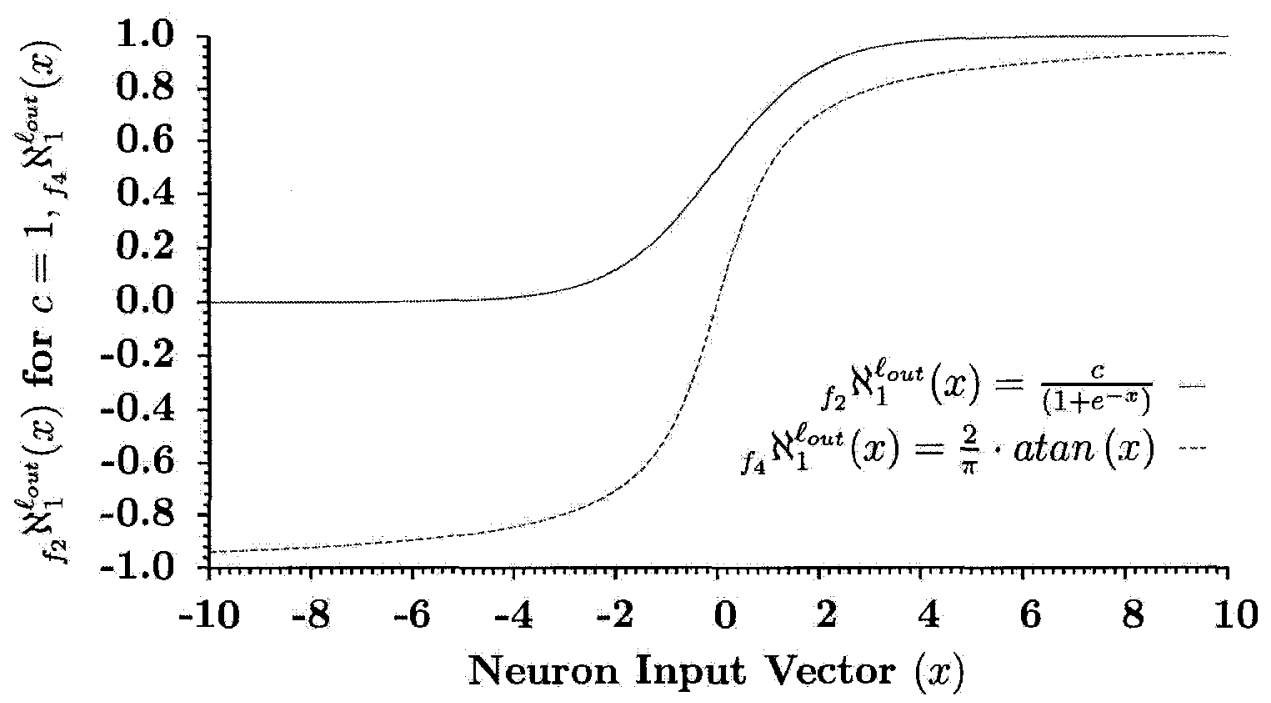

Figure 4.6: Example of the difference between two classical activation functions.

logistic). The classical logistic function is usable if a scaler is applied. For example, Fig. 4.7 on page 71 demonstrates 3 possible mapping functions $(f: R \rightarrow[0 . . c])$ on two examples (when $c=2$ and when $c=5$ ). For the case $(c=2)$ no biases ${ }^{3}$ amongst the 3 functions can be seen. However, for the case $(c=5)$ it can be clearly seen that the classical logistic function has a bias towards the first and last classes after the mapping has been performed, while the other two functions do not. Before any experimentation is performed, any sort of bias seems like an undesirable characteristic.

\subsubsection{Terminals and Primitive Functions}

Classical neural network design uses a fixed neural network architecture (e.g. NN:51-2, with fixed activation and aggregation functions. The learning occurs through the use of a modifiable weight value, which is classically learned via the backpropagation

\footnotetext{
${ }^{3}$ Bias is measured by the length of the curve within a region for a particular class compared to the length of the curve for the other classes. For example, in Fig. 4.7 on page 71 for Case $c=5$, the plane constrained to the $y$ values in $[1 . .2]$ is shorter than the infinite length of the curve in $[0 . .1]$. To be pedantic, the endpoints belong to only one class.
} 


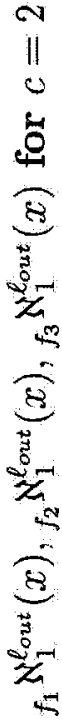

$$
\begin{array}{r}
f_{1} \aleph_{1}^{\ell_{\text {out }}}(x)=\left|c \cdot \frac{2}{\pi} \cdot \operatorname{asin}\left(\sin \left(\pi \cdot x-\frac{\pi}{2}\right)\right)\right|- \\
{ }_{f_{2}} \aleph_{1}^{\ell_{\text {out }}}(x)=\frac{c}{\left(1+e^{-x}\right)} \\
f_{3} \aleph_{1}^{\ell_{\text {out }}}(x)=\frac{c}{2} \cdot \sin (c \cdot \pi \cdot x)+\frac{c}{2} \cdots
\end{array}
$$

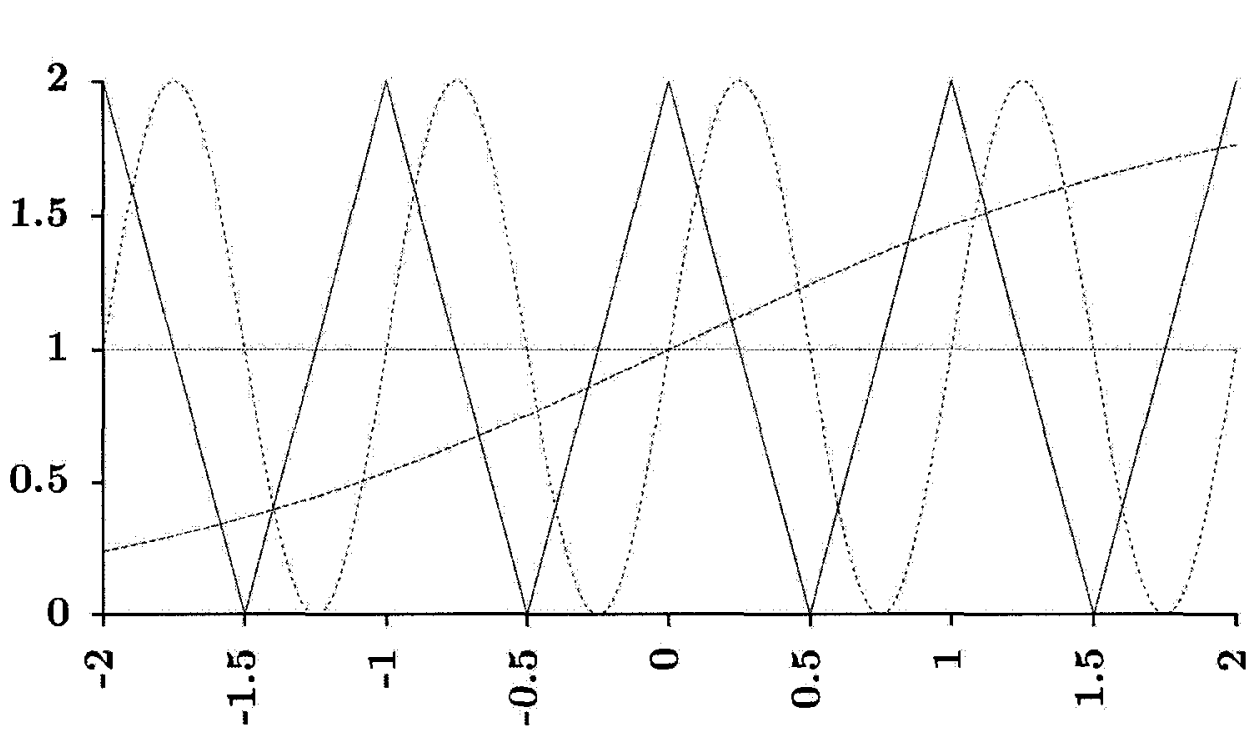

Neuron Input Vector $(x)$

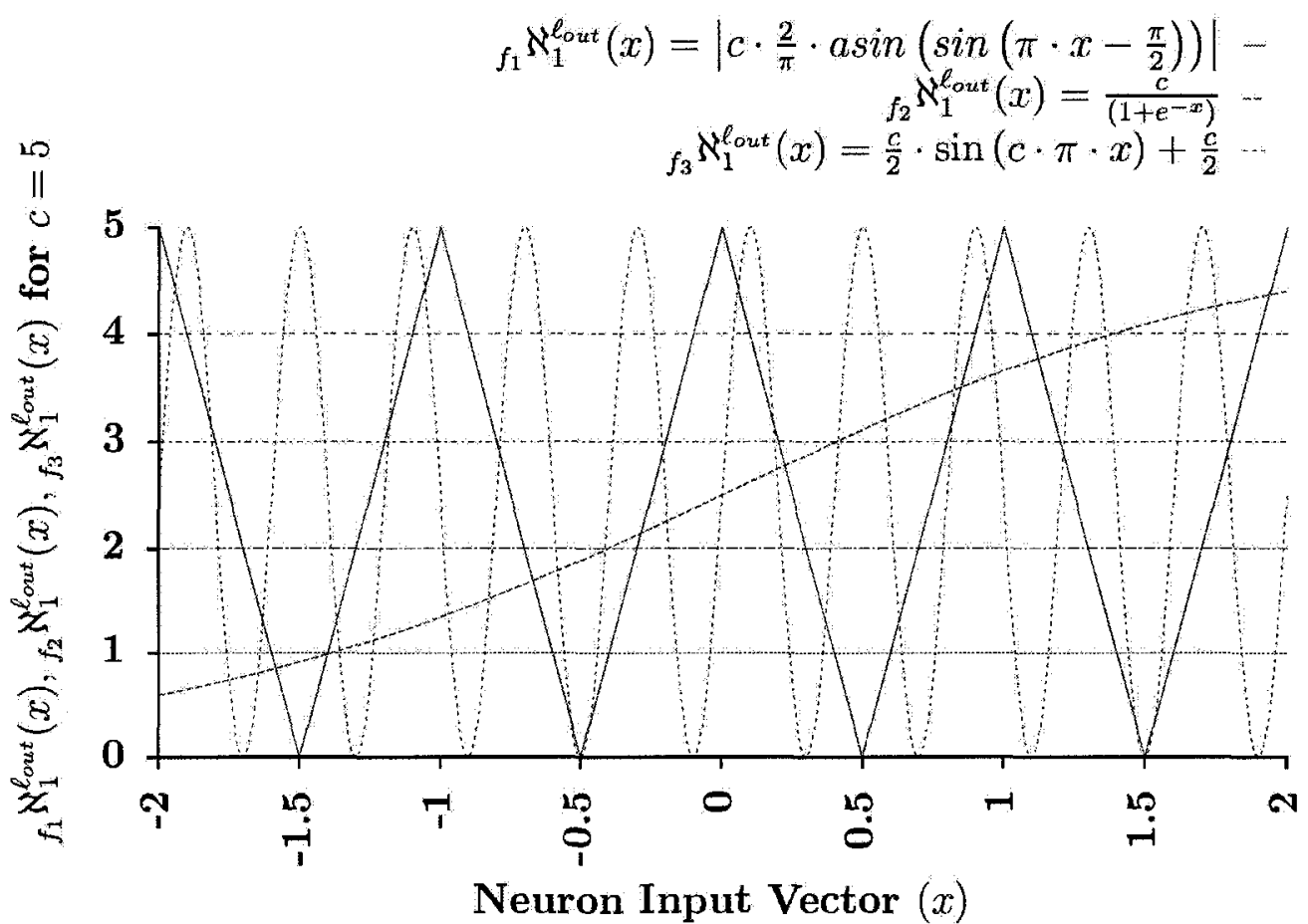

Figure 4.7: When deciding between possible output neuron mappings, 3 were selected for consideration and are demonstrated on two example cases $(c=\{2,5\})$.

Top: Case $c=2$ in which all 3 functions have no bias towards a particular class. Bottom: Case $c=5$ in which $f_{3} \aleph_{1}^{\ell_{\text {out }}}(x)$ is biased towards class 1 or class $5, f_{2} \aleph_{1}^{\ell_{\text {out }}}(x)$ has continuous derivatives but awkward amplitude locations, and $f_{1} \aleph_{1}^{\ell_{\text {out }}}(x)$ has period 1 and is piecewise linear with derivative discontinuities. 
algorithm. For example, an aggregation function could be the sum function that adds the weighted outputs of the neurons that are giving input to the current neuron. The outputs of each neuron is controlled by its specific activation function, which is classically kept the same for all neurons and could be, for example, the logistic function (See Fig. 4.6 on page 70).

Classical neural network learning does not learn anything other than the weights on the neuron outputs. This thesis expands the learning paradigm in order to learn the complete neuron function. That is, both the aggregation of neuron outputs including which neurons or subset of neurons to use and the activation function, specifying how the aforementioned outputs should be combined in order to produce the current neuron's output, are learned. The possible set of functions to use within each neuron is conceptually unbounded. In addition, there is no reason that the set of possible functions within neuron $i$ should be the set of possible functions within neuron $j$. For practical reasons, this thesis considers that all neurons may only select their functions (terminals and primitive functions) from those that have been implemented within the system (See Appendix B on page 181 for the possibilities). John Koza performed experiments by first selecting the simplest primitive functions for consideration. This thesis follows that approach (See Table 6.6 on page 104).

\subsection{Fitness Measure for an Individual}

There is a one-to-one correspondence between chromosomes and neurons. The evolutionary machinery of constructing new individuals will result in new chromosome values (i.e. new genes within the associated genome) and hence new neuron functions over time (See Fig. 4.4 on page 67 ). Now, at a given point in time, when an individual's fitness needs to be ascertained the neural network needs to be evaluated for its performance on the training data (testing data is a different matter and is deferred to Section 4.5 on page 76). In summary, GEP is being used as a global optimizer in order to minimize the neural network error on training data.

How is the performance of the particular neural network determined (i.e. the fitness of the individual)? This involves pushing each training object through the network and determining the class to which the network predicts the object belongs. 
After all of these classes have been predicted, a comparison may be made to the expected classes because all objects in the training data have associated classes. How should such a comparison between the set of predicted classes and the set of expected classes be made? There are many ways. This thesis considers two of them $\mathcal{E}_{C}$ and $\mathcal{E}_{C_{N}}$; that is, classification error and a normalized classification error. Once such an error measure $(\mathcal{E})$ is decided upon and measured with respect to the particular individual under consideration, then a fitness measure may be reported. How should the error measure be used as a fitness measure? Again, there are many possibilities. This thesis only uses one as shown in Eq. 4.1 on page 73, which is used in order to map a particular error measure (this theses uses $\mathcal{E}_{C}$ and $\mathcal{E}_{C_{N}}$ ) from the interval [0..1] to the interval [0.1000]. This is an arbitrary restriction by the particular software being used. Fitness values of 1000 indicate that an individual is ideal (i.e. that a satisfactory solution has been found). The details of floating point arithmetic within a computer would come into consideration during fitness evaluation because an individual that does not have 0 error (e.g. 0.0000001) may also be considered to be a solution (i.e. ideal) and may indicate that algorithm termination should occur. In addition, a complication arises for error values that cannot be computed. For example, a divide by zero within the function associated to a neuron will result in that neuron having an undefined value ( $\mathrm{NaN}$ ) and potentially resulting in the neural network having an undefined error $(\mathcal{E})$ value. Such an undefined fitness for an individual will be converted by the software implementation into a zero fitness value. Hence, such individuals that cannot be evaluated will still recombine with others in the population according to the algorithm configuration.

$$
\mathcal{I}_{\text {fit }}=(1.0-\mathcal{E}) \cdot 1000.0
$$

\subsubsection{Error Measures $(\mathcal{E})$ used within Fitness}

As mentioned, there are many possible error measures. Each of them provides a different facet computed from the associated confusion matrix. When the data has 2 classes, the confusion matrix in Table 4.1 on page 74 may be considered. It can be seen that certain entries have names while others do not. For example, entry 4 , which is an object belonging to class $c_{1}$ cannot be classified by the classifier. These 
Table 4.1: Example 2 Class Problem Confusion Matrix.

\begin{tabular}{c|ccc} 
known predicted & Unclassifiable & $c_{1}$ (Positive) & $c_{2}$ (Negative) \\
\hline Unknown & 1 & 2 & 3 \\
$c_{1}$ (Positive) & 4 & True Positive (TP)False Negative (FN) \\
$c_{2}$ (Negative) & 5 & False Positive (FP)True Negative (TN)
\end{tabular}

names may be used to construct common summaries [Lu et al., 2004] of the confusion matrix as in Table 4.2 on page 75. Of course, these measures must be extended when considering larger numbers of classes and hence a larger confusion matrix. The error measures considered in this thesis are shown in Eq. 4.3 on page 74. $\mathcal{E}_{C}$ is the classical classification error measure and $\mathcal{E}_{C_{N}}$ is a normalized version.

To be more verbose, $\mathcal{E}_{C}$ is an error measure that is derived from the ratio of the number of incorrectly classified objects to the total number of objects. The numerator may be computed by summing over all classes. The items in the sum are the differences between the number of objects per class and the number of correctly predicted objects per class. $\mathcal{E}_{C_{N}}$ is normalized within the sum (i.e. on a per class basis) rather than over all objects as is done for $\mathcal{E}_{C}$. It is helpful to draw Venn diagrams to understand this situation more clearly.

This thesis calculates $\mathcal{E}_{C}^{T r}, \mathcal{E}_{C}^{T e}, \mathcal{E}_{C_{N}}^{T r}$ and $\mathcal{E}_{C_{N}}^{T e}$ within each fitness evaluation but only uses one of $\mathcal{E}_{C}^{T r}$ or $\mathcal{E}_{C_{N}}^{T r}$ for the particular optimization under consideration.

$$
\begin{aligned}
\mathcal{E}_{C} & =\frac{\sum_{i=1}^{c}\left(\operatorname{card}\left(\mathrm{C}_{i}^{\exp }\right)-\operatorname{card}\left(\mathrm{C}_{i}^{\exp } \cap \mathrm{C}_{i}^{\text {obs }}\right)\right)}{\operatorname{card}(\mathrm{Obj})} \\
\mathcal{E}_{C_{N}}= & \frac{\sum_{i=1}^{c}\left(\operatorname{card}\left(\mathrm{C}_{i}^{\mathrm{exp}}\right)-\operatorname{card}\left(\mathrm{C}_{i}^{\exp } \cap \mathrm{C}_{i}^{\mathrm{obs}}\right)\right) / \operatorname{card}\left(\mathrm{C}_{i}^{\exp }\right)}{c}
\end{aligned}
$$

\subsection{Controlling the Algorithm Search}

The evolutionary algorithm may search through the fitness landscape in many ways. The values of the parameters that are given as input to the algorithm are basically instructions telling the algorithm how to make choices. For example, the settings in 
Table 4.2: Common 2 Class Problem Error Measures. http://www.cs . ualberta.ca/ eisner/measures.html

\begin{tabular}{|c|c|c|}
\hline Measure & Equation & Intuitive Meaning \\
\hline Precision & $\frac{T P}{T P+F P}$ & $\begin{array}{l}\text { The percentage of positive } \\
\text { predictions that are correct. }\end{array}$ \\
\hline Recall/Sensitivity & $\frac{T P}{T P+F N}$ & $\begin{array}{l}\text { The percentage of positive } \\
\text { labeled instances that were } \\
\text { predicted as positive. }\end{array}$ \\
\hline Specificity & $\frac{T N}{T N+F P}$ & $\begin{array}{l}\text { The percentage of negative } \\
\text { labeled instances that were } \\
\text { predicted as negative. }\end{array}$ \\
\hline Accuracy & $\frac{T P+T N}{T P+T N+F P+F N}$ & $\begin{array}{l}\text { The percentage of predic- } \\
\text { tions that are correct. }\end{array}$ \\
\hline
\end{tabular}

Table 6.6 on page 104 provide a large number of instructions informing the algorithm what to do in various situations; such as how many chromosomes should be used for each individual, or how should genes be linked together for a particular chromosome. This set of variable parameters constitutes a parameter space. This thesis varies 11 parameters, and results are reported relating the 11 dimensional parameter space to the class of "good" and "bad" neural network solutions".

One of the main points of this thesis, is that GEP may be used to learn the functions within a neural network. But what form should the network take? This thesis investigates feed forward networks that have a fixed number of layers and a fixed number of neurons per layer. This thesis allows the feed forward connections between these neurons to be learned. That is, an upper bound on the connections between layer $i$ and layer $i+1$ is enforced in the sense that only those neurons within layer $i$ may be used within functions for neurons within layer $i+1$. The neuron in layer $i$ may be used 0 or more times within any of the neurons within layer $i+1$. The true upper bound, then, is determined by the maximum number of terminals that are allowable. But how does one chose what the upper bound should be? This is always a question within neural network research. For example, work on choosing the optimal neural network size for searching through large image databases has been

\footnotetext{
${ }^{4} \mathrm{~A}$ good neural network solution is problem dependant and may be defined in many ways. This thesis takes the simplest approach and uses a discrimination threshold.
} 
done [Messer and Kittler, 1998]. General approaches are presented, which including growing and pruning techniques. To be specific, growing techniques start with a small network and increase the complexity through the addition of neurons or neuron connections until the network performs adequately, while pruning techniques start with a very large network that is fully trained and then attempt to remove redundant weights and neurons without losing the overall networks performance. The problem with the latter approach is that a fully trained network needs to already exist.

Previous experience with the particular data sets within this thesis allow the choice of a potentially appropriate neural network architecture [Valdés and Barton, 2006], [Valdés and Barton, 2007]. That is, i) the number of neurons within the first layer is fixed to be the number of input variables, $i i)$ the number of neurons within the last layer is fixed to be either one neuron or c neurons, iii) the number of layers is fixed to be three, and $i v$ ) the number of neurons within the hidden layer is fixed to be the estimated intrinsic dimensionality of the variable space.

\subsection{Terminating the Algorithm: A Heuristic Approach}

The NN-GEP algorithm will terminate if one of the following cases is satisfied: $\left\{\begin{array}{l}\text { Case I, the pre-specified maximum number of generations has been reached; } \\ \text { Case II, the error measure }(\mathcal{E}) \text { increases on testing data. }\end{array}\right.$

For both cases, the optimization process (See Alg. 1 on page 14) is oriented towards maximizing fitness, which is related to minimizing training error $\left(\mathcal{E}_{C}^{T r}\right.$ or $\left.\mathcal{E}_{C_{N}}^{T r}\right)$. For Case II, the error measures considered within this thesis are $\mathcal{E} \in\left\{\mathcal{E}_{C}^{T e}, \mathcal{E}_{C_{N}}^{T e}\right\}$; others are possible. In Alg. 1 on page 14, the archive, $\mathcal{A}$, is initialized to be empty on Line 1 and is updated on Line 4 and Line 9 . Every time an update is made, the current minimum archived training error result, $\mathcal{I}_{\text {best }} \in \mathcal{A}$, is compared to $\mathcal{I}_{\text {best }}^{\prime} \in \mathcal{P}=\mathcal{F}_{\mathcal{P}}$. That is, if (trainingError $\left(\mathcal{I}_{\text {best }}^{\prime}\right)<\operatorname{trainingError}\left(\mathcal{I}_{\text {best }}\right)$ ) and (testingError $\left(\mathcal{I}_{b e s t}^{\prime}\right)>$ testingError $\left(\mathcal{I}_{\text {best }}\right)$ ) then Case II is satisfied, and termination should occur. The intuition behind Case II is that the population of "good" individuals are not generalizing on test data, so it is assumed that NN-GEP is overfitting the problem. 
Table 4.3: Example demonstrating NN-GEP termination issues.

\begin{tabular}{cccccc}
\hline Generation & Individual & $\begin{array}{c}\text { Train Error } \\
\left(\mathcal{E}_{C}^{T r} \text { or } \mathcal{E}_{C_{N}}^{T r}\right)\end{array}$ & $\begin{array}{c}\text { Test Error } \\
\left(\mathcal{E}_{C}^{T e} \text { or } \mathcal{E}_{C_{N}}^{T e}\right)\end{array}$ & Relative Test Error & \\
\hline \hline 1 & 3 & 0.20 & $(0.20)$ & $?$ & cont. \\
\hline 4 & 5 & 0.10 & 0.11 & $0.11 / 0.20=0.55$ & cont. \\
4 & 6 & 0.10 & $(0.10)$ & $0.10 / 0.20=0.50$ & cont. \\
\hline 7 & 2 & 0.04 & 0.15 & $0.15 / 0.10=1.50$ & stop \\
7 & 8 & 0.05 & $(0.09)$ & $0.09 / 0.10=0.90$ & cont. \\
7 & 11 & 0.04 & 0.15 & $0.15 / 0.10=1.50$ & stop \\
\hline \hline
\end{tabular}

\subsubsection{Example of Termination based on Testing Error $\left(\mathcal{E}_{C}^{T e}\right.$ or $\left.\mathcal{E}_{C_{N}}^{T e}\right)$}

Consider the example evolution listed in Table 4.3 on page 77. Also, assume 10 individuals in the population and assume individuals are evaluated one after another (sequential processing). In this example, the complete population has to be considered before a decision to stop (or not) is made because, as in Table 4.3 on page 77, two (or more) individuals in the same population may indicate contradictory advice with respect to continuing or terminating the evolutionary process. Perhaps this issue would be addressed sooner if a parallel algorithm, rather than sequential algorithm, would be used. However, a chosen design for the parallel algorithm may be to process the solution results as they arrive on a first-come first-served basis, hence, reducing the problem back to one of sequential processing of the individuals but with a random ordering of individuals from the population rather than a fixed ordering.

\subsection{Enhancements compared to a Standard GEP Implementation}

This thesis extends previous work [Valdés et al., 2007]. Previous work had a global set of symbols (terminals, primitive functions). That means that each chromosome within an individual has the same set of available symbols. This thesis extended the concept in such a way that it now becomes possible to have a different set of symbols associated to each chromosome ${ }^{5}$. The second extension over the standard GEP implementation is the modification to enable a population based termination criteria, rather than a criteria solely based upon an individual. The standard GEP

\footnotetext{
${ }^{5}$ The name of the java class, within the implementation, is GEPSymbolsets.
} 
Table 4.4: Example of the effect of errors on termination criteria. To address practical data collection from the generated NN-GEP results database, the "best" data entry is re-written to disk. In generation 11, one individual has better training error than all previous training errors, but worse testing error. No other individual in that generation had an improvement in training error. The algorithm may also be terminated when the maximum number of generations has been reached.

\begin{tabular}{cccccc}
\hline \hline Generation & Individual & $\begin{array}{c}\text { Train Error } \\
\left(\mathcal{E}_{C}^{T r} \text { or } \mathcal{E}_{C_{N}}^{T r}\right)\end{array}$ & $\begin{array}{c}\text { Test Error } \\
\left(\mathcal{E}_{C}^{T e} \text { or } \mathcal{E}_{C_{N}}^{T e}\right)\end{array}$ & Relative Test Error & \\
\hline \hline 1 & 3 & 0.20 & $(0.20)$ & $?$ & cont. \\
\hline 4 & 5 & 0.10 & 0.11 & $0.11 / 0.20=0.55$ & cont. \\
4 & 6 & 0.10 & $(0.10)$ & $0.10 / 0.20=0.50$ & cont. \\
\hline 7 & 2 & 0.04 & 0.15 & $0.15 / 0.10=1.50$ & stop \\
7 & 8 & 0.05 & $(0.09)$ & $0.09 / 0.10=0.90$ & cont. \\
7 & 11 & 0.04 & 0.15 & $0.15 / 0.10=1.50$ & stop \\
\hline $11^{*}$ & 5 & 0.45 & 0.10 & $0.10 / 0.09=1 . \overline{1}$ & stop \\
\hline 7 & 8 & 0.05 & $(0.09)$ & $0.09 / 0.10=0.90$ & cont. \\
\hline \hline
\end{tabular}

algorithm terminates based upon a specified number of generations being reached or upon an ideal solution being found. The concept of an ideal solution is such that if a particular fitness measure indicates that an individual is ideal, then the evolutionary process should be prematurely terminated. This thesis presents an extension to a population based termination criteria. In particular, Table 4.3 on page 77 gives an example and Table 4.4 on page 78 specifies exactly what was implemented within the software. That is, an additional data row will be written to the results file that repeats the best solution of the generation (See Table 4.4 on page 78) in order to simplify the collection of the results by a utility program, by ensuring that the last data entry will always be the best solution even in the case of premature termination.

In addition to generalizing the GEP algorithm in two ways, this thesis implements a specific fitness measure by taking advantage of the symbol set extension mentioned previously. That is, a neural network may now be constructed in such a way that the set of available terminals within the first layer neurons (i.e. chromosomes) use the input variables, while the set of terminals in the second layer neurons only use terminals that are related in a one-to-one manner with the previous layer neurons. That is, the output of, say, neuron 3 in layer 2 produces a value $\left(o_{3}^{\ell_{2}}\right)$ that may be 
assigned to a terminal (12n3out), while neuron 4 in layer 2 would produce a value $\left(o_{4}^{\ell_{2}}\right)$ that would be assigned to terminal (12n4out). These terminals are within the set of available terminals within each neuron $1,2, \ldots$ in layer 3 .

\subsection{Chapter Summary}

This chapter presented the classification problem and that this thesis focuses on a neural network classifier that is more transparent than the classical networks. This transparency is in the form of analytic functions that can be derived from the network and used independently of the training procedure. An additional advantage of selecting a neural network representation is to be able to construct mapping functions as well as classifiers. The theoretical details of how the network is represented within an individual's multiple chromosomes were presented along with specific enhancements to the GEP algorithm. The implementation details are now of concern. 


\section{Chapter 5}

\section{NN-GEP Software Engineering and Design}

The current age of computers has given rise to a field of study called Software Engineering that involves abstract concepts about how to describe the design of a program that is capable of solving a particular problem $(\mathbb{P})$. For programs that are mainly algorithmic in nature, then a specification may be used (e.g. for a specification of a program that creates programs see Alg. 1 on page 14). Such algorithmic specifications are very general but may not be appropriate in some cases ${ }^{1}$. The Software Engineering field provides more than one perspective, for example, in terms of models of the software implementation [OMG, 2008]. This chapter does not provide all possible perspectives for NN-GEP, but attempts to select a subset in which some of the important details are revealed. In particular, an algorithmic specification, class diagrams and sequence diagrams of the software system (ECJ-16 [Luke et al., 2008]) are reported.

\subsection{Discussion of Design Issues}

A software system (ECJ-16 [Luke et al., 2008]) existed and was extended in order to evolve neuron functions within a neural network (See Section 4.6 on page 77). A lot of documentation on the system exists online and so will not be repeated in this thesis. The intention of the following subsections is to demonstrate:

1. that the system is quite complex and may be used for a variety of purposes; by giving as an example, the GEP class diagram (a visual model),

2. how the system was extended; by providing sequence diagrams, and

3. how the fitness measure was implemented; by providing visual models.

\footnotetext{
${ }^{1}$ e.g. specificity may be lost due to a need to keep the specification concise
} 


\subsubsection{The Fitness Function (Processing Algorithm)}

A general evolutionary computation algorithm has been previously specified (See Alg. 1 on page 14); within it, the measure of fitness for an individual (See Section 4.3 on page 72) is the main processing function. For NN-GEP, it is Alg. 2 on page 82, which is also described in terms of a sequence diagram in Fig. 5.5 on page 87 and Fig. 5.6 on page 88 .

\subsubsection{GEP Class Diagram}

UMLGraph [Spinellis, 2003] was used throughout this chapter and was used to construct the GEP class diagram shown in Fig. 5.1 on page 83. More precisely, the following enhancements were made to the implementation:

1. GEPIndividual was enhanced to support getChromosomeAt (int index), returning a GEPChromosome.

2. GEPChromosome was enhanced to support getSymbolSetForChromosome(), returning a GEPSymbolset. It was also enhanced to support the functions GEPSymbol getSymbolAt (int index) and GEPSymbol getTerminalAt (int index) so that the caller does not need to know to what GEPSymbolset the GEPChromosome is associated.

3. GEPSpecies was extended to support an array of instances of GEPSymbo1Set, which may be retrieved by name, from, for example, GEPChromosome because each chromosome has a name associated with it (i.e. each neuron is a chromosome and has a name).

4. The derived classes of the general GEPSymbol class are GEPTerminalSymbol and GEPFunctionSymbol; with GEPConstantTerminalSymbol a grandchild.

5. FerreiraGeneHeadStrategy was extracted from the code and now derives from a new interface GEPGeneHeadStrategy, in order to support future strategies.

6. NN-GEP extends GEPProblem in order to provide a very specific fitness implementation that is not provided within GEPFitnessFunction, which provides a set of static, already implemented, functions. 


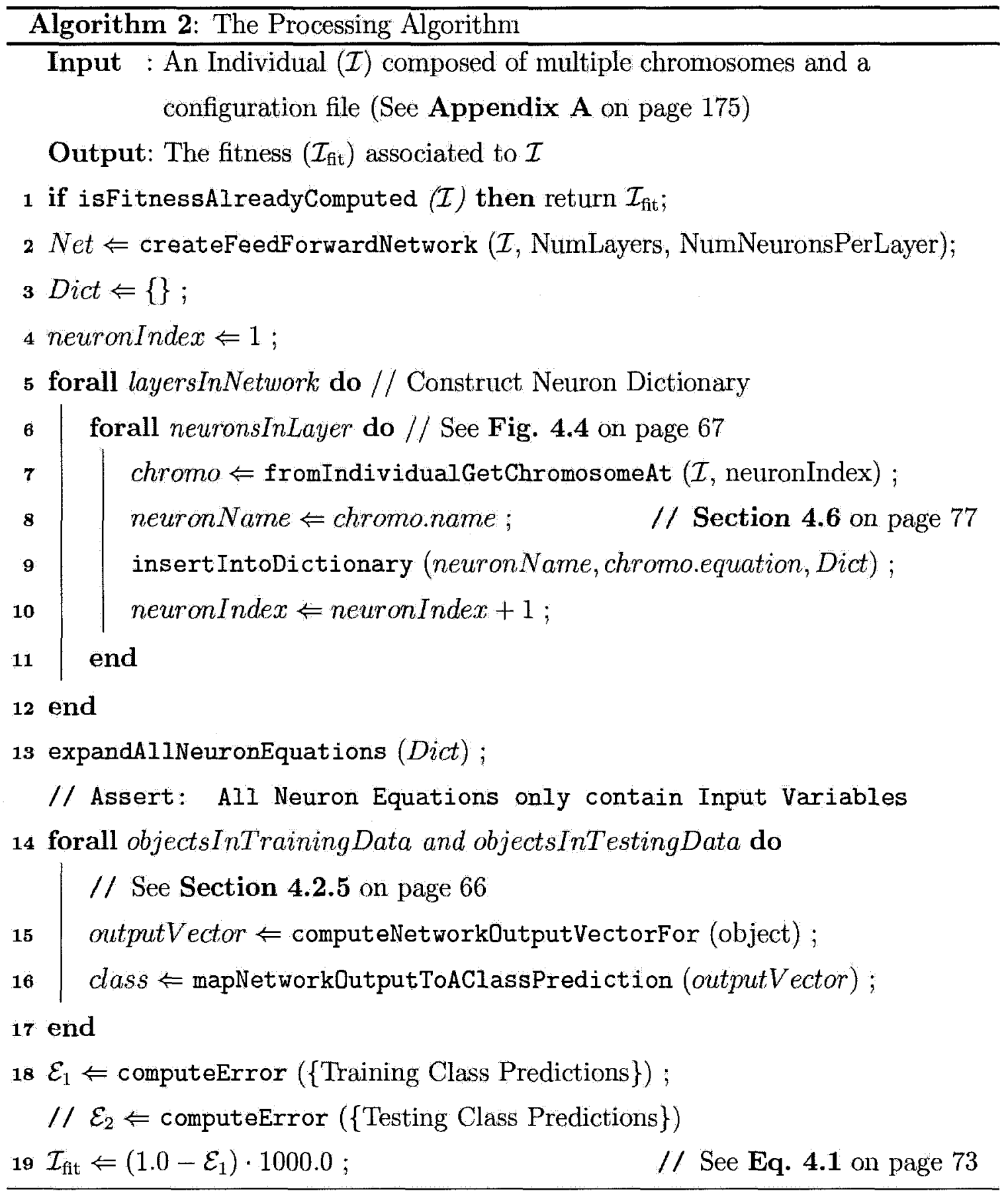

7. NN-GEP needs the following set of classes, which had to be implemented within GEP: FeedForwardNeuralNetwork, AbstractError, ClassificationError, SumOfResidualsError, PrimitiveFloatStack, and PrimitiveStringStack. 


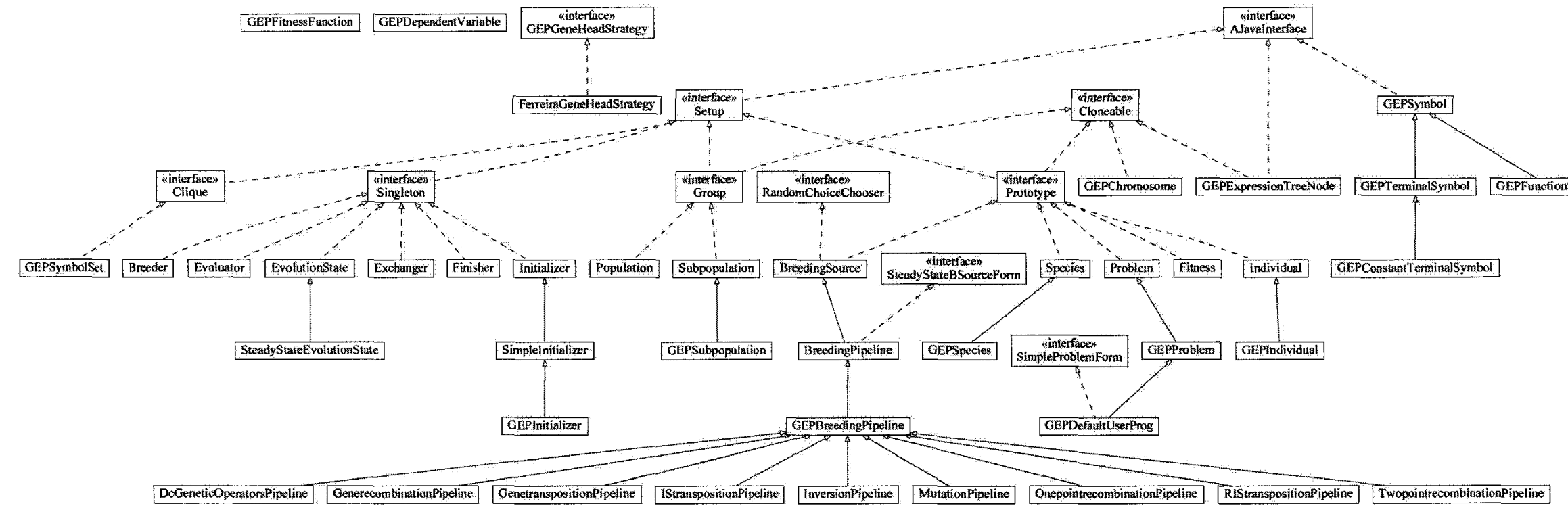

Figure 5.1: A selected subset of the classes and interfaces within ECJ-16 [Luke et al., 2008] that are relevant to GEP. UMLGraph was used to generate this visual representation. It is easy to see that many classes are needed in order to implement the GEP algorithm. However, it is difficult to understand the details; such as temporal relationships. (For details, see Fig. 5.2 on page 84, Fig. 5.3 on page 85, Fig. 5.4 on page 85, Fig. 5.5 on page 87, Fig. 5.6 on page 88) 


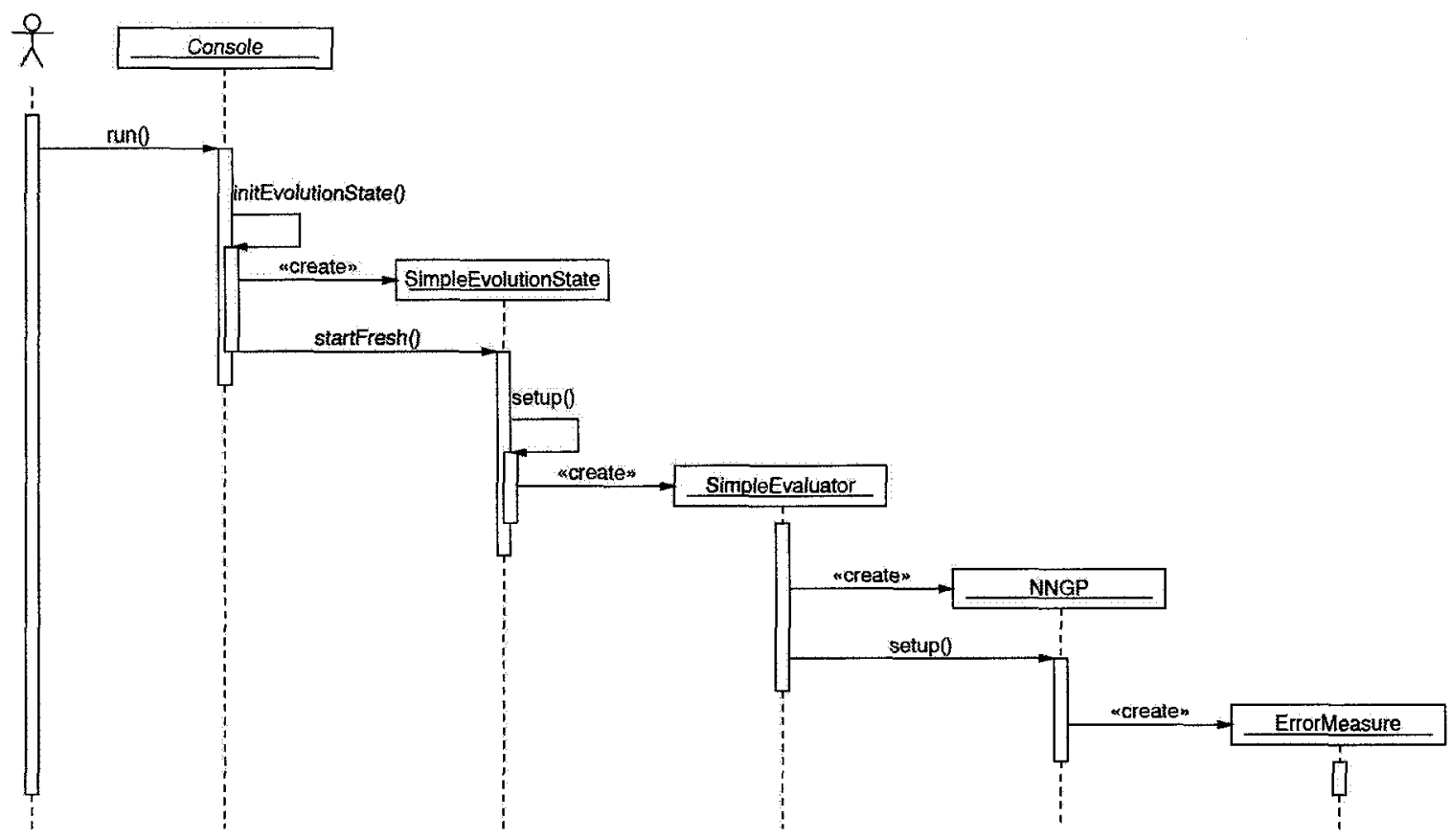

Figure 5.2: Sequence Diagram detailing how, in general, ECJ initializes the various base classes for use by any evolutionary computation algorithm (e.g. GEP).

\subsubsection{NN-GEP Sequence Diagrams}

When the user starts the program running from the console, the sequence of events are shown in Fig. 5.2 on page 84. In particular, 4 instances are created, including the NN-GEP instance, which initializes itself from the control parameters that were read from a file and passed to the function. For an example of the NN-GEP configuration file, see Appendix A on page 175.

Fig. 5.3 on page 85 shows the sequence of events when the first population is constructed and then initialized. The ECJ class SimpleEvolutionstate instructs the GEPInitializer class to create a population of a certain size. For GEP, there is only one population, represented as a GEPSubpopulation. Other algorithms within ECJ support multiple populations, such as an island model that co-evolves populations. Of course, GEP does not know how to find the fitness associated to each individual in this initial population, so NN-GEP is asked for that information; depending on the controlling parameters within Appendix A on page 175.

In Section 2.2.4 on page 22, the algorithm was shown to go through an evolutionary process. The first step has already been explained, whereby an initial population 


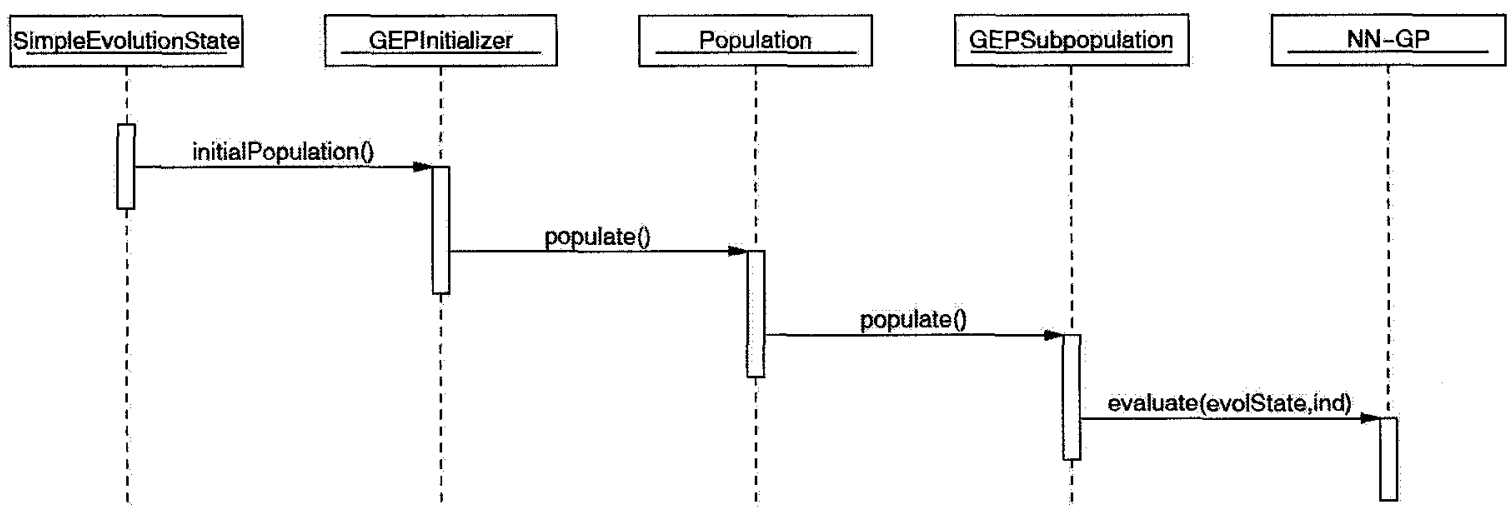

Figure 5.3: Sequence Diagram detailing how the initial population is constructed.

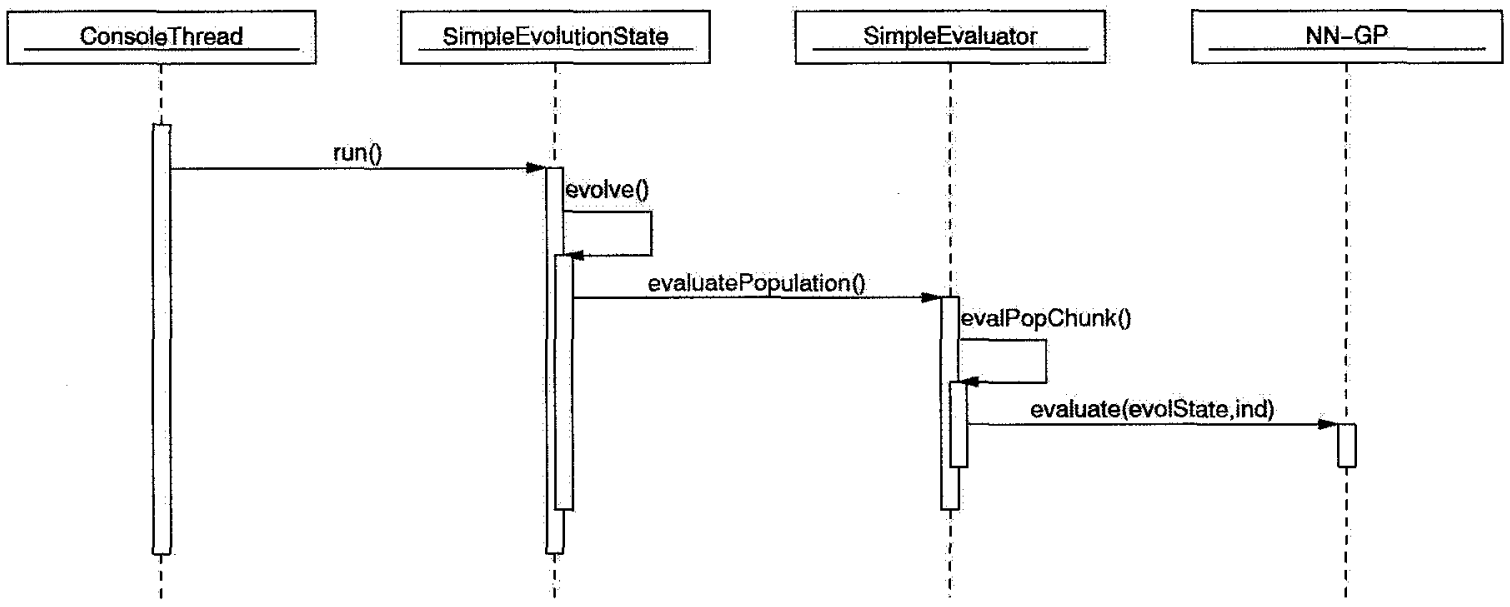

Figure 5.4: Sequence Diagram detailing which classes are involved when an individual in the population is evaluated during the evolutionary process.

of individuals is constructed. The second, and main step, is to progress through a series of generations in which a population is modified via various (GEP) operators in order to create a new set of individuals. The specific controlling parameters (See Appendix A on page 175) determine precisely what actions are performed by GEP, but the actual fitness is problem dependant. In this case, NN-GEP is used to evaluate a specific individual in the population.

There are quite a few steps that are involved in evaluating an individual, which are shown in Fig. 5.5 on page 87 and Fig. $\mathbf{5 . 6}$ on page 88 . Let us walk through the details. In the first case, NN-GEP is asked to evaluate the fitness of an individual, which has already been evaluated in the past. Therefore, this evaluation may return the previously calculated value and the evolution may proceed. In the second case, the 
fitness must be evaluated. NN-GEP retrieves the fitness framework (SimpleFitness) provided by GEP and then creates the FeedForwardNeuralNetwork instance. The neural network architecture is known from the configuration file (See Appendix A on page 175) and so a neuron dictionary is constructed by looping over the layers and neurons. When the $i^{\text {th }}$ chromosome within the individual is retrieved, the neuron name is constructed (e.g. see Section 4.6 on page 77) and the chromosome equation is retrieved. The neuron name and equation are placed into the dictionary.

The dictionary is now in a state where each neuron has an associated expression that uses terminals from the previous layer ${ }^{2}$ only. The terminals need to be expanded into their associated expressions. This is done by a replace string operator that removes a neuron name and places its associated expression into the string. The result of this variable substitution is that each neuron then has an associated expression that is only in terms of the input variables.

The neural network output now needs to be computed for each object in the training data and each object in the testing data for the measures under consideration (see Section 4.3.1 on page 73). This is done by evaluating each neuron when the terminals are replaced with their respective values. Once this has completed, the errors may be computed based on the neural network outputs and NN-GEP may archive the result if it exceeds the best of anything previously seen. Of course, termination criteria may be satisfied if an ideal solution is found or if all of the individuals in the current generation are not decreasing training and testing errors (see Section 4.5 on page 76). The instance of NN-GEP is then destroyed and, if appropriate, the next individual is considered.

\footnotetext{
${ }^{2}$ or input variables if it is the first layer
} 


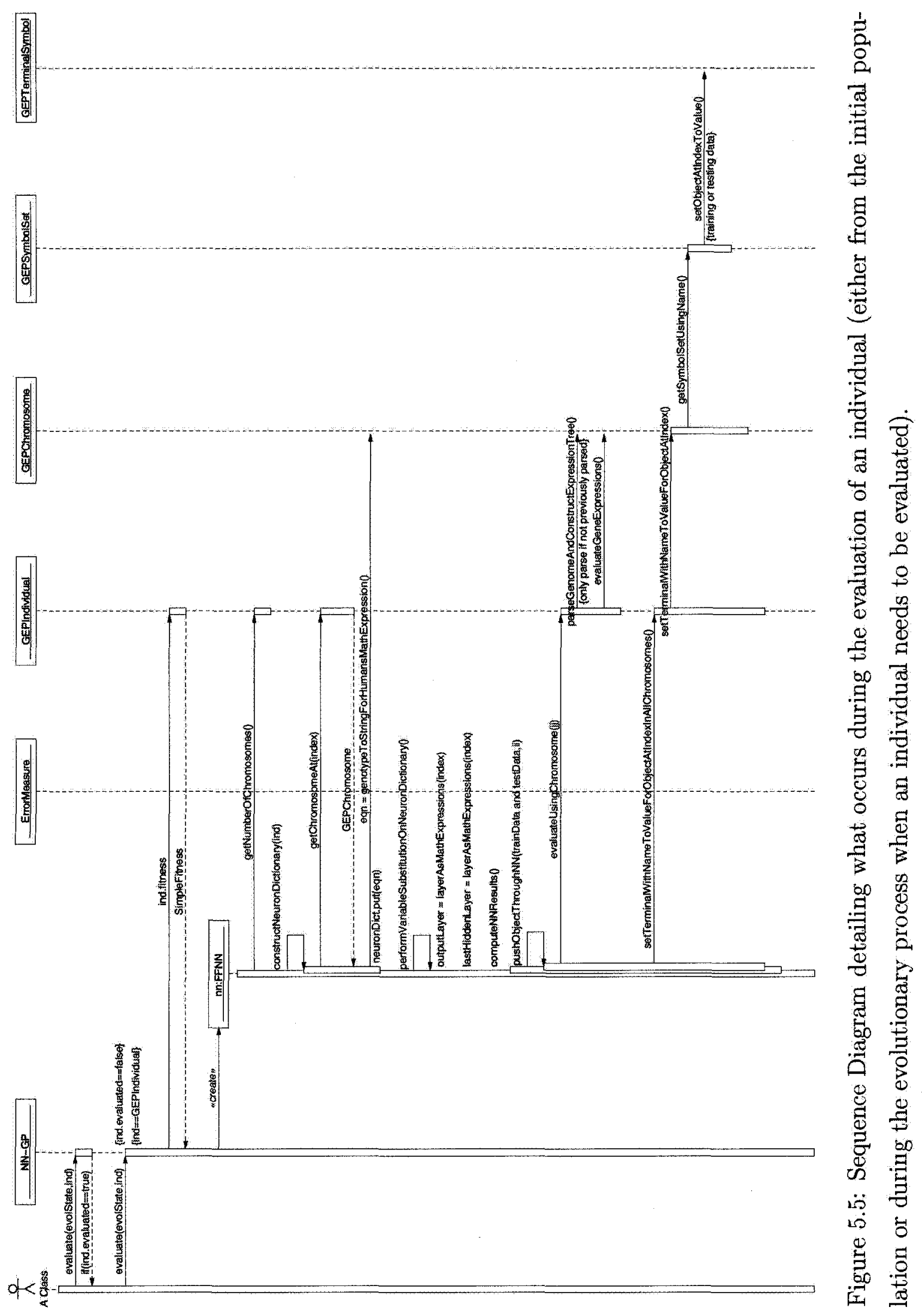



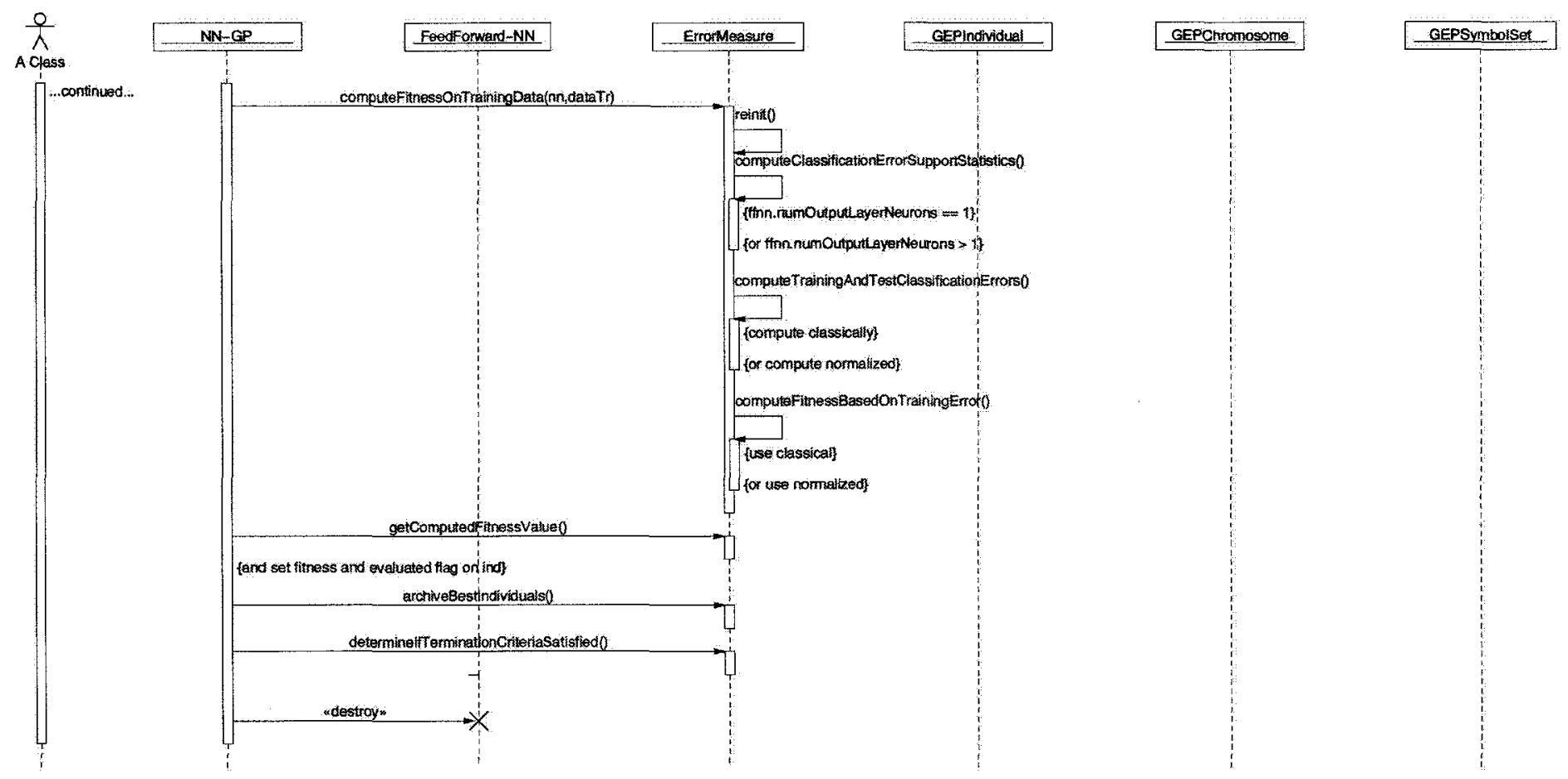

GEPTErminalsymbol

Figure 5.6: Sequence Diagram continuing the detailing of what occurs during the evaluation of an individual. 


\subsection{Chapter Summary}

This chapter has presented the most relevant implementation details for NN-GEP, including the classes involved in the computation of an individual's fitness. A neuron dictionary plays a key role in aiding the neural network computation. In addition, reporting of the equations associated with each neuron is essential if experimentation is to proceed. The next chapter starts this methodology by discussing the settings that will be investigated within the experiments. 


\section{Chapter 6}

\section{Experimental Procedures and Testing Methodology}

The theory and design of NN-GEP have been previously discussed. This chapter focusses on describing what aspects of the algorithm will be investigated. This is done by presenting information about 4 chosen data sets that each contain different properties that are expected to uncover NN-GEP's strengths and weaknesses; for no algorithm is a panacea. The choice of experiment dictates what is possible to report in terms of results. This thesis takes a parsimonious approach and avoids the design of an optimal experiment (e.g. [Sacks et al., 1989], [Eiben and Jelasity, 2002]), but rather chooses to broadly and evenly sample a subset of the parameter space, which is potentially more relevant. This thesis uses Ferreira's experimentally found default values for GEP and investigates several other combinations.

\subsection{The Data Descriptions}

A set of 3 real world and 1 publicly available data sets are described; for 2 types of experiments. The first type of experiments are for a broad scale investigation and NN-GEP settings are shown in Table 6.6 on page 104, while the second type of experiments are for a more focussed investigation on a much larger dimensional problem with multiple classes and the settings are shown in Table 6.4 on page 101 . This second investigation was not done in such a broad scale because it was estimated to require $\approx 126.25$ years of computation time (See Section 6.2.4.1 on page 106).

\subsubsection{Broad Scale Experiment Settings: Insunza $(c=1)$}

Earth Sciences research in Geophysical Prospecting has been performed in which geophysical data was obtained from an investigation dealing with the detection of underground caves [Valdés and Barton, 2006]. Karstification is a peculiar geomorphological and hydrogeological phenomenon produced by rock solution as the dominant 


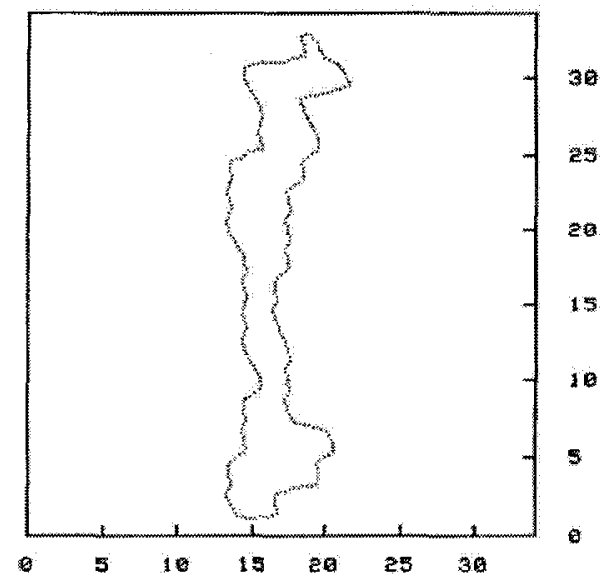

Figure 6.1: Location of the known Insunza Cave. Units are 2D measuring station indices.

process. As a consequence, the earth's surface is covered by irregular morphologies, like lapiaz, closed depressions(dolinas), sinks, potholes and underground caves. The hydrographic network is usually poorly developed and rain waters infiltrate to form an underground drainage system. Sometimes the caves are opened to the surface, but typically they are buried, requiring the use of geophysical methods. Cave detection is a very important problem in civil and geological engineering.

The studied area contained an accessible cave (Fig. 6.1 on page 91) and geophysical methods complemented with a topographic survey were used with the purpose of finding their relation with subsurface phenomena [Valdés and Gil, 1982]. This is a problem with partially defined classes: the existence of a cave beneath a measurement station is either known for sure if made over the known cave, or unknown since there might be a buried cave beneath. Accordingly, only one class membership is defined.

In the area, a gentle variation in geological conditions for both the bedrock and the overburden was suspected by geologists. An isolation of the different geophysical field sources was necessary in order to focus the study on the contribution coming from underground targets, in an attempt to minimize the influence of both the larger geological structures and the local heterogeneities. 
Table 6.1: Description of the variables (i.e. set of geophysical methods) within the Insunza data (See Fig. 6.2 on page 92).

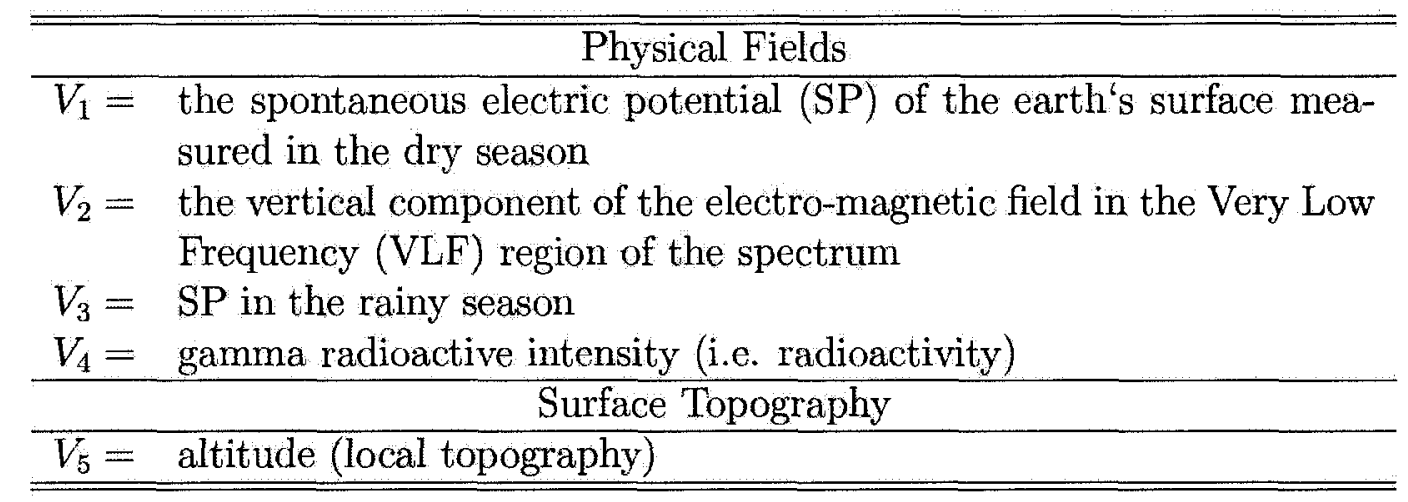

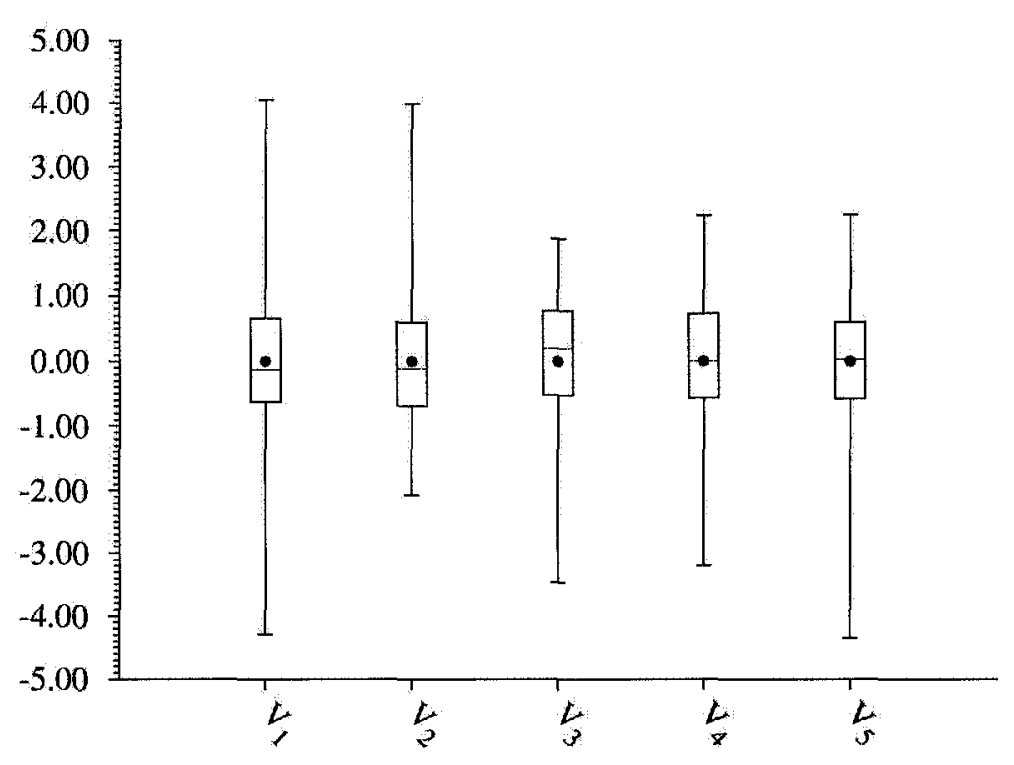

Four quartiles and mean for each variable $(\mathrm{x})$

Figure 6.2: Box and whisker plots for each variable within the z-scored (mean=0, stdev =1) Insunza data [Valdés and Barton, 2006]. The plot has been augmented with a dot representing the mean. (See Table 6.1 on page 92) 


\subsubsection{Insunza Data Preprocessing}

The complexity of these measured geophysical fields in the area is illustrated by the distribution of the standard scores corresponding to the gamma ray intensity and the surface topography. While radioactivity is highly noisy, topography shows few features. Both fields are shown in Fig. 6.3 on page 93 (left and right). Since the geophysical fields are measured using different units, in order to neutralize the effect of the different scales introduced by the units of measurement, all values were transformed to standard scores (i.e. to variables with zero mean and unit variance).
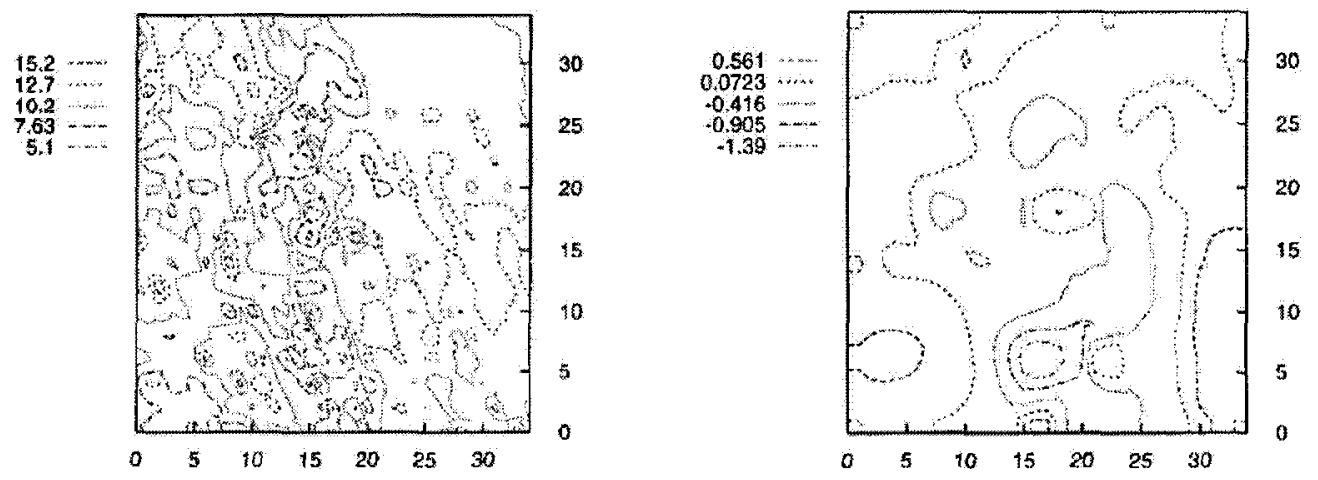

Figure 6.3: Left: Distribution of the gamma ray intensity (standard scores). Right: Local topography (standard scores).

Each geophysical field was assumed to be described by the following additive two-dimensional model composed of trend, signal, and random noise: $f(x, y)=$ $t(x, y)+s(x, y)+n(x, y)$. where $f$ is the physical field, $t$ is the trend, $s$ is the signal, and $n$ is the random noise component, respectively. To isolate an approximation of the signals produced by the underground target bodies, a linear trend term $\hat{t}(x, y)=c_{0}+c_{1} x+c_{2} y$ was fitted (by least squares) and subtracted from the original field. The residuals $\hat{r}(x, y)=f(x, y)-\hat{t}(x, y)$ were then filtered by direct convolution with a low pass finite-extent impulse response two-dimensional filter to attenuate the random noise component. Such convolution is given by $\hat{s}(x, y)=$ $\sum_{k_{1}=-N}^{N} \sum_{k_{2}=-N}^{N} h\left(k_{1}, k_{2}\right) \hat{r}\left(x-k_{1}, y-k_{2}\right)$ where $\hat{r}(x, y)$ is the residual, $\hat{s}(x, y)$ is the signal approximation, and $h\left(k_{1}, k_{2}\right)$ is the low-pass zero-phase shift filter.

The collection of residual fields (as an approximation to the signals for all physical 
Table 6.2: Description of the variables within the Breast Cancer data (See Fig. 6.4 on page 95 ).

\begin{tabular}{l}
\hline$V_{1}=$ Clump thickness. \\
$V_{2}=$ Uniformity of Cell Size. \\
$V_{3}=$ Uniformity of Cell Shape. \\
$V_{4}=$ Marginal Adhesion. \\
$V_{5}=$ Single Epithelial Cell Size. \\
$V_{6}=$ Bare Nuclei. \\
$V_{7}=$ Bland Chromatin. \\
$V_{8}=$ Normal Nucleoli. \\
$V_{9}=$ Mitoses. \\
\hline
\end{tabular}

fields) was used for analysis. In total, 1225 points in a regular grid were measured for the five physical fields previously mentioned.

\subsubsection{Broad Scale Experiment Settings: UCI Breast Cancer $(c=2)$}

The Magnetic Resonance Spectra (See Section 6.1.4 on page 97) was estimated to take $\approx 126.25$ years to compute (See Section 6.2.4.1 on page 106) within the broad scale methodology (See Section 6.2 on page 100), therefore a publicly available data set was chosen that had published and related results that could be contrasted [Brown, 2004]. The University of California, Irvine (UCI) maintains an international machine learning (ML) database repository [Asuncion and Newman, 2007], containing an archive of over 100 databases used specifically for evaluating machine learning algorithms. For evaluating NN-GEP within the broad scale experiments, a breast cancer database was obtained [Wolberg and Mangasarian, 1990]. It contains 699 objects, 9 integer-valued attributes and is composed of 2 classes (241 malignant and 458 benign). In addition, 16 objects contain missing values, which, for the purposes of this study, were removed from consideration (leaving 683 objects).

\subsubsection{Broad Scale Experiment Settings: Werenskiold $(c=5)$}

Hydrochemical Research in the Arctic has been performed [Valdés and Barton, 2007] that is based on a scientific expedition in 1985 (Spitzbergen'85) organized by the University of Silesia in Poland, a scientific team composed by specialists from this 


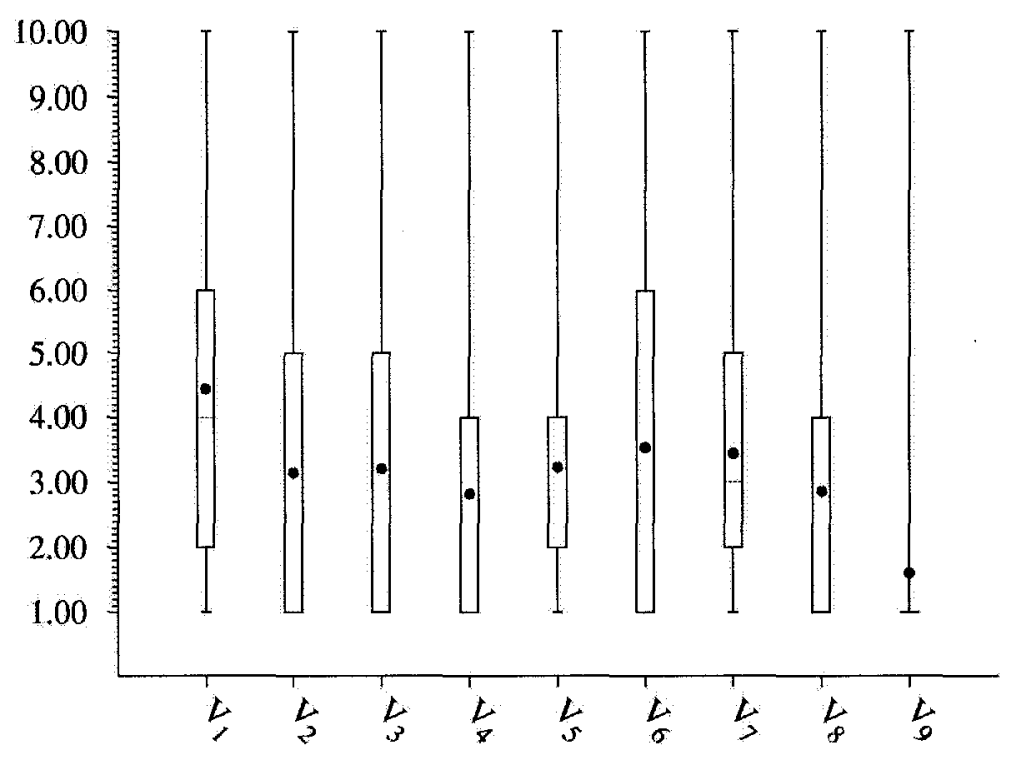

Four quartiles and mean for each variable (x)

Figure 6.4: Box and whisker plots for each variable within the Breast Cancer data [Wolberg and Mangasarian, 1990]. The plot has been augmented with a dot representing the mean.

university, the National Center for Scientific Research Cuba, and the Academy of Sciences of Cuba in which they performed glaciological and hydrogeological investigations in several regions of the Spizbergen island in the Svalbard archipielago Fig. 6.5 on page 96.

The purpose of the research was to determine the mass and energy balance within experimental hydrogeological basins, to study the interaction between natural waters and rock forming minerals under the severe conditions of polar climate and the comparison with similar processes developed in tropical conditions. This has been a long term research of several polish universities (Silesia, Warsaw and Wroclaw) and the Polish Academy of Sciences since the First Geophysical Year in 1957. This research has made an important contribution to the evaluation of the impact of global climatic changes. In this region there are complex processes taking place under peculiar geological, geomorphological and hydrogeological conditions which are reflected in water geochemistry. During that expedition, a collection of ice and water samples were taken from different hydrogeological zones in the region of the Hornsund 


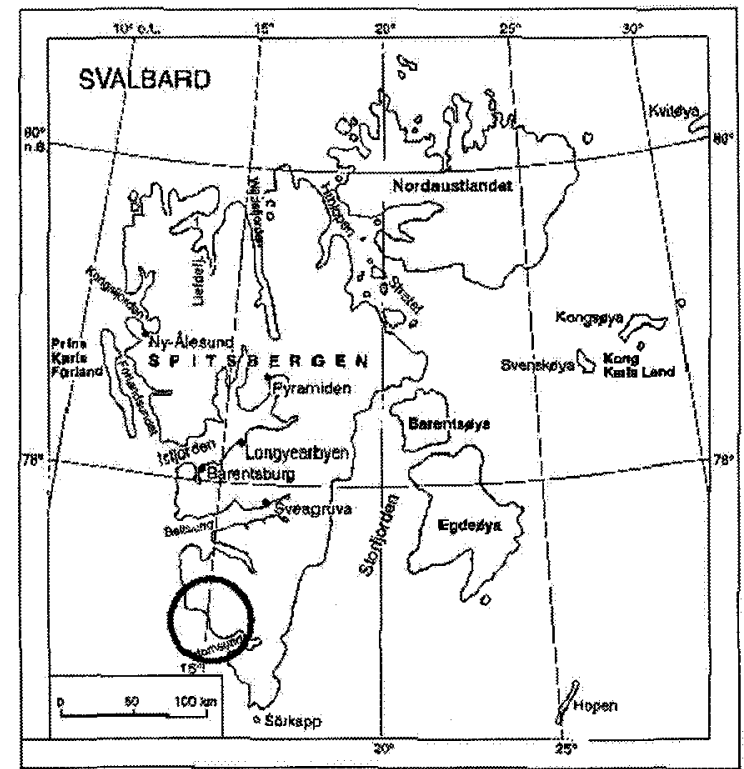

Figure 6.5: The Svalbard archipelago (Spitzbergen is the main island). The area where the samples were taken is the neighborhood of the Werenskiold Glacier, to the north-west of the Hornsund Fiord (highlighted with a circle).

fjords in Spitzbergen, specifically in the basin of the Werenskiold Glacier Fig. 6.5 on page 96. These samples are representative of different zones from a hydrogeological point of view: subglacial, superglacial, springs, lakes, snow, ice, and the tundra. Geochemical and hydrogeological studies of these data [Fagundo et al., 1990], [Fagundo et al., 1996] have shown the relation between the different hydrogeological conditions present in Spitzbergen and the chemical composition of the waters and ice. This is reflected by the existence of several families of waters, thus enabling an indirect assessment of their hydrogeological origin from the information given by their geochemical parameters. A set of water samples from Spitzbergen corresponding to 5 hydrogeological families of waters were characterized by a collection of physical and chemical parameters which were determined in situ (See Fig. 6.6 on page 97 and Table 6.3 on page 97 ). 


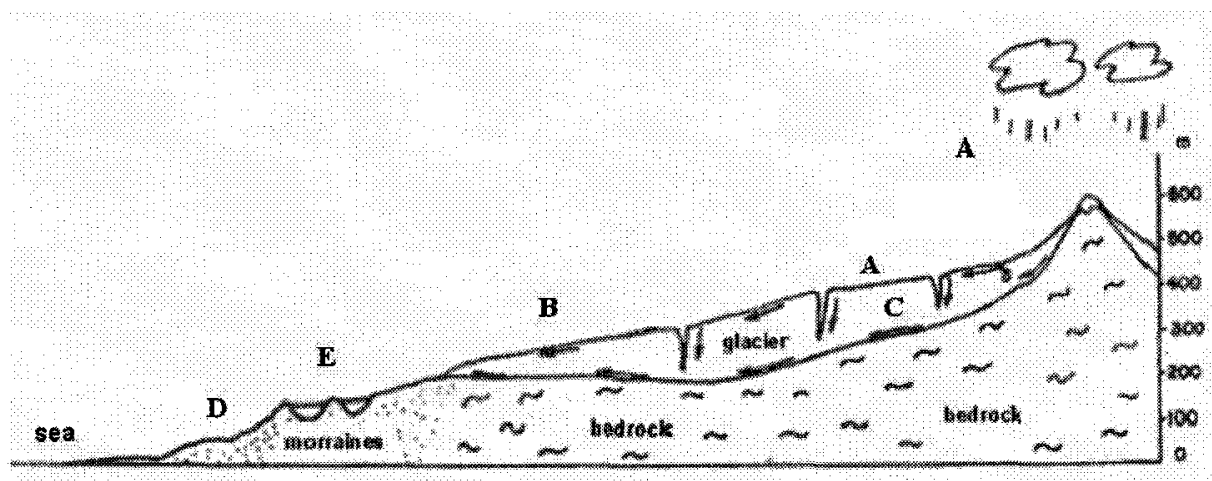

Figure 6.6: Longitudinal section of the Werenskiold Glacier. The different types of waters analyzed are: A) precipitation (snow, ice), B) superglacial, C) subglacial, D) tundra, E) moraine lakes.

Table 6.3: Description of the variables within the Werenskiold data (See Fig. 6.7 on page 98 ).

\begin{tabular}{ll}
\hline \hline$V_{1}=$ temperature. \\
$V_{2}=$ pH. \\
$V_{3}=$ electrical conductivity. \\
$V_{4}=$ hydrocarbonate. \\
$V_{5}=$ chloride. \\
$V_{6}=$ sulphate. \\
$V_{7}=$ calcium. \\
$V_{8}=$ magnesium. \\
$V_{9}=$ sodium-potasium. \\
\hline \hline
\end{tabular}

\subsubsection{Published: Magnetic Resonance Spectra $(c=4)$}

Magnetic resonance spectroscopy (MRS) and magnetic resonance imaging (MRI) are two non-invasive and harmless clinical techniques that can provide useful biochemical information about a region of interest in the body [Barton and Valdés, 2008]. They can be particularly helpful when the organ under investigation is difficult or dangerous to reach (e.g. the brain) where direct inspection and surgery should be avoided as much as possible. Both techniques are based on magnetic resonance (MR), which is related to the physical property called quantum spin. The MRI technique reveals water concentration levels and is used in routine examinations by clinicians; whereas the MRS technique is not used as frequently as MRI (despite its great potential). MRS information consists of a signal, possibly noisy, composed of peaks 


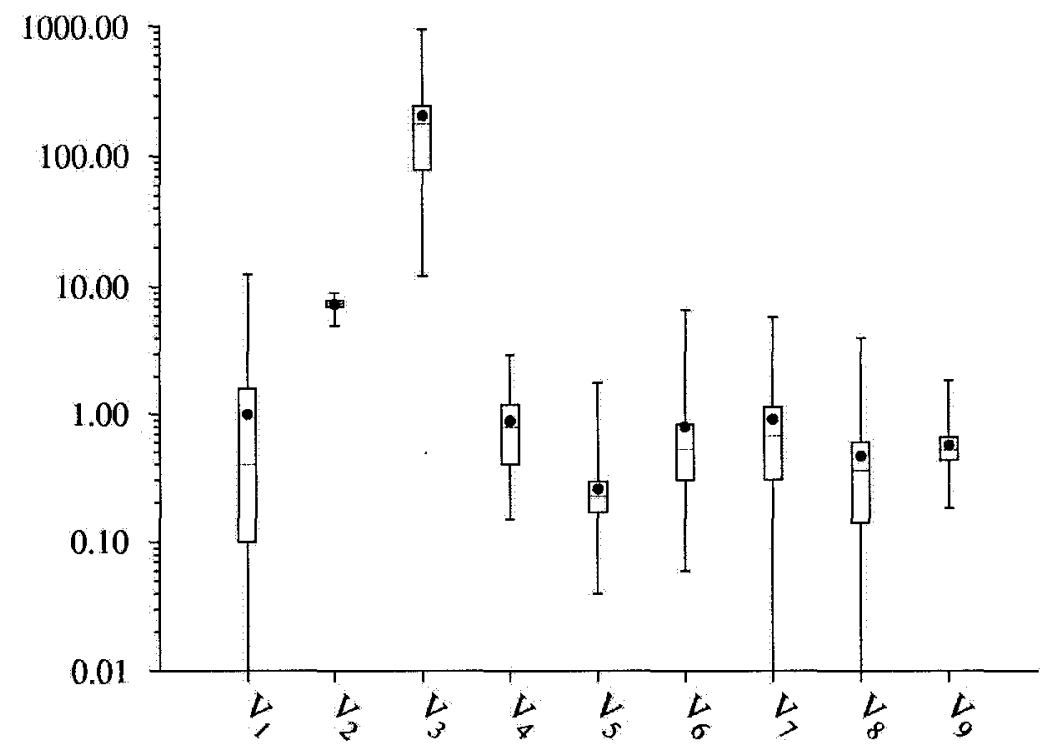

Four quartiles and mean for each variable $(\mathrm{x})$

Figure 6.7: Box and whisker plots for each variable within the Werenskiold data [Valdés and Barton, 2007]. The plot has been augmented with a dot representing the mean. Electrical conductivity has a much broader range than all of the others because water samples are from snow, ice and rain, which have very small electrical conductivity (water without minerals in it), to ocean water, which is highly conductive (large amount of dissolved minerals (salts)).

whose location and height correspond to different metabolites and their relative concentrations. Reading the most frequent chemical in an MR spectrum is relatively straightforward, but the complete interpretation of a spectrum or the comparison between two spectra usually requires an expert [Ladroue, 2004]. This reliance on specialized expertise may be one of the reasons why it has been more difficult to introduce MRS into routine medical practice. An international project, INTERPRET http://azizu.uab.es/INTERPRET, gathered the efforts of 5 centers across Europe with the long term goal of generalizing the use of MRS. During this project, a large database of $1 \mathrm{HMR}$ spectra was built in order to develop an automatic MRS-based system to aid clinicians to diagnose brain tumors. Each spectrum in the database was acquired according to a pre-defined protocol and formally validated by clinicians and pathologists [Juliá-Sapé et al., 2006].

The height and shape of each resonance in the MR spectrum is determined by 


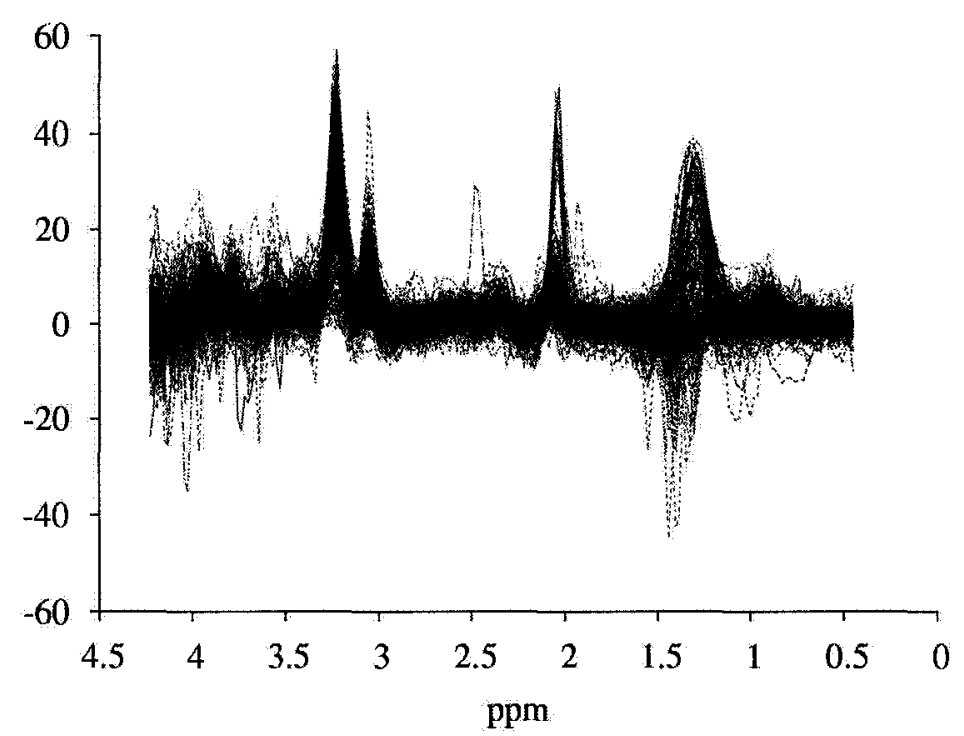

Figure 6.8: All Long Echo Time (TE $\geq 130 \mathrm{~ms}$ ) MR Spectra from 285 patients.

several parameters related to the way in which signal produced by the exited proton spin decays by a relaxation process. One of them, called the echo time (TE) is very important. The longer the $\mathrm{TE}$, the more the signal has attenuated before acquisition. Hence, a short echo time spectrum ( $\mathrm{TE} \leq 50 \mathrm{~ms}$ ) has larger peaks than a long echo time spectrum ( $\mathrm{TE} \geq 130 \mathrm{~ms}$ ). A short echo time spectrum also contains more peaks, as resonances with a small relaxation value or complex coupling pattern, like $\mathrm{mI}$ (myo-Inositol), Glu (glutamate) and Gln (glutamine) are less pronounced at longer echo times. At short echo time signals, macromolecules are prominent; originating from proteins and membrane components. They have very broad peaks with a large contribution to an underlying and partially unknown baseline [Ladroue, 2004], [Devos, 2005].

The data used in this study consist of 219 long-echo MRS spectra (echo time $\mathrm{TE} \geq 130 \mathrm{~ms})$. The data acquisition protocol and the signal processing procedure is described in [Tate et al., 2006]. Each spectrum covers a range between [4.23 .. 0.45 ] parts per million (ppm) along the $\mathrm{x}$-axis, where 200 equally spaced samples were taken. The set represents different types of tumors and normal cases grouped into 4 main classes: G1: astrocytome, oligoastrocytome and oligodendrogliome, G2: glioblastome and metastasis and G3: meningiomes. Those tumors represent the 
major groups within the available validated data but this thesis has a preliminary character and so will focus on the study of the tumor vs normal differentiation (i.e. $\{G 1, G 2, G 3\}$ vs \{normal $\}$ ), with 204 and 15 cases respectively.

In order to simplify the application of some procedures, in particular genetic programming, the dataset (219 individuals and 200 predictive variables) was linearly re-scaled from its original range $[-44.850571,56.267685]$ to the $[1,100]$ range. The purpose was to work with strictly positive values and since the target range is almost the same as the original (99 vs 101.118256), the re-scaling operation is essentially a shifting. The re-scaled data was divided into a training and a test set using random stratified sampling so that class proportions were preserved. The training set contained $80 \%$ of the data ( 175 objects) and the test set the remaining $20 \%$ (44 objects).

The NN-GEP approach was investigated within a series of two experiments (See Table 6.4 on page 101). The first series of 240 attempted to broadly sweep the parameter space; with the second series of 2,250 being used to more closely investigate the parameter space around the good solution obtained within the first series.

\subsubsection{Summary of All Experimental Data Sets}

The class and object frequencies for all of the investigated data sets are reported in Table 6.5 on page 102. There are 4 columns. The first, data set, identifies the name and number of classes. The second, original, reports the number of objects and attributes for the complete data and the number of objects on a per class basis. The third and fourth columns, constructed training and constructed testing are similar descriptions for 2 data sets that were constructed from the original data by random object selection without replacement.

\subsection{The Broad Scale Experimental Methodology}

Based on information learned from preliminary experiments, a broad scale methodology was developed for more thoroughly investigating NN-GEP's performance on particular data sets. Alg. 3 on page 103 shows the two main stages; a sequential, and a distributed portion. For the sequential portion, a specific machine, usually the local host, is used to perform some preliminary processing as it only needs to be 
Table 6.4: MRS Experimental settings for the two series of experiments involving NN-GEP that have been published. See Table 6.6 on page 104 for the broad scale experimental settings.

\begin{tabular}{l|l|l}
\hline \hline & Series 1 (240) & Series 2 (2250) \\
\hline GEP Max. Num. Generations & 50 & same \\
GEP Population Size & $5,10,15$ & 10 \\
GEP Num. Elite Individuals & 1 & same \\
GEP Inversion Rate & 0.1 & same \\
GEP Mutation Rate & 0.044 & same \\
GEP IS Transposition Rate & 0.1 & same \\
GEP RIS Transposition Rate & 0.1 & same \\
GEP One Point Recomb. Rate & 0.3 & same \\
GEP Two Point Recomb. Rate & 0.3 & same \\
GEP Gene Recombination Rate & 0.1 & same \\
GEP Gene Transposition Rate & 0.1 & same \\
GEP Num. Genes Per Chromosome & 1 & same \\
GEP Gene Headsize & 2 & same \\
GEP Gene Linking Function & Addition & same \\
GEP Num. Real Constants Per Gene & $2,4,8,200$ & $1,2,3,4,5$ \\
GEP Constants Limits & {$[-100.0,100.0]$} & same \\
GEP Seeds & 5 unique seeds & Series 1 and 45 more \\
GEP Species RNC Mutation Rate & 0.01 & same \\
GEP Species DC Mutation Rate & 0.044 & same \\
GEP Species DC Inversion Rate & 0.1 & same \\
GEP Species DC IS Transposition Rate & 0.1 & same \\
\cline { 2 - 3 } GEP Functions For All Symbol Sets & Addition, Subtraction, Multiplication \\
GEP Number of Symbol Sets & Determined by NN topology: 3 (one/layer) \\
GEP Number of Chromosomes & Determined by NN topology: 202 \\
Neural Network (NN) Topology & 200 Input Nodes, 1 Hidden, 1 Output \\
\cline { 2 - 3 } NN Input Layer Constant Weights & 1,200 & $1,100,200$ \\
NN Input Layer Terminal Weights & 1 & same \\
NN Hidden Layer Constant Weights & 1,200 & $1,100,200$ \\
NN Hidden Layer Terminal Weights & 1 & same \\
NN Output Layer Constant Weights & 1 & same \\
NN Output Layer Terminal Weights & 1 & same \\
\hline \hline
\end{tabular}

performed once. The data is then distributed via a specific distribution mechanism to a set of waiting compute nodes, which may include the distributing machine. Once all of the computations have completed, the sequential portion of the pipeline may then proceed to collect the results from all of the files that have been placed back 
Table 6.5: Investigated data sets. Training \& testing sets were constructed by random object selection without replacement.

\begin{tabular}{|c|c|c|c|}
\hline Data Set & Original & $\begin{array}{c}\text { Constructed } \\
\text { Training }\end{array}$ & $\begin{array}{c}\text { Constructed } \\
\text { Testing }\end{array}$ \\
\hline \multirow[t]{2}{*}{ Insunza } & $((\operatorname{card}(\mathrm{Obj})=1,225)$ by $(\operatorname{card}(\mathrm{Attr})=5))$ & $(\operatorname{card}(\mathrm{Obj})=981)$ & $(\operatorname{card}(\mathrm{Obj})=244)$ \\
\hline & $<1104_{c_{1}}, 121_{c_{2}}>$ & $<884_{c_{1}}, 97_{c_{2}}>_{\operatorname{Tr}}$ & $<220_{c_{1}}, 24_{c_{2}}>_{\mathrm{Te}}$ \\
\hline \multirow{2}{*}{$\begin{array}{l}\text { UCI Breast Cancer } \\
\qquad(c=2)\end{array}$} & $((\operatorname{card}(\mathrm{Obj})=683)$ by $(\operatorname{card}($ Attr $)=9))$ & $(\operatorname{card}(\mathrm{Obj})=548)$ & $(\operatorname{card}(\mathrm{Obj})=135)$ \\
\hline & $<444_{c_{1}}, 239_{c_{2}}>$ & $<356_{c_{1}}, 192_{c_{2}}>_{\operatorname{Tr}}$ & $<88_{c_{1}}, 47_{c_{2}}>_{\mathrm{Te}}$ \\
\hline \multirow[t]{2}{*}{ Werenskiold } & $((\operatorname{card}(\mathrm{Obj})=114)$ by $(\operatorname{card}($ Attr $)=9))$ & $(\operatorname{card}(\mathrm{Obj})=94)$ & $(\operatorname{card}(\mathrm{Obj})=20)$ \\
\hline & $<37_{c_{1}}, 29_{c_{2}}, 10_{c_{3}}, 11_{c_{4}}, 27_{c_{5}}>$ & $<30_{c_{1}}, 24_{c_{2}}, 9_{c_{3}}, 9_{c_{4}}, 22_{c_{5}}>_{\mathrm{Tr}}$ & $<7_{c_{1}}, 5_{c_{2}}, 1_{c_{3}}, 2_{c_{4}}, 5_{c_{5}}>_{\mathrm{Te}}$ \\
\hline \multicolumn{2}{|c|}{ Magnetic Resonance $((\operatorname{card}(\mathrm{Obj})=266)$ by $(\operatorname{card}(\mathrm{Attr})=200))$} & $(\operatorname{card}(\mathrm{Obj})=175)$ & $(\operatorname{card}(\mathrm{Obj})=44)$ \\
\hline \multirow{3}{*}{$\begin{array}{l}\text { Spectra } \\
\text { (long echo only) } \\
\qquad(c=15)\end{array}$} & $<55_{c_{1}}, 78_{c_{2}}, 31_{c_{3}}, 20_{c_{4}}, 6_{c_{5}}, \cdots>$ & $<12_{c_{1}}, 163_{c_{2}}>_{\mathrm{Tr}}$ & $<3_{c_{1}}, 41_{c_{2}}>_{\mathrm{Te}}$ \\
\hline & $<5_{c_{6}}, 18_{c_{7}}, 3_{c_{8}}, 6_{c_{9}}, 9_{c_{10}}, \cdots>$ & & \\
\hline & $<7_{c_{11}}, 3_{c_{12}}, 8_{c_{13}}, 2_{c_{14}}, 15_{c_{15}}>$ & & \\
\hline
\end{tabular}


onto the original distributing machine. The resultant databases may then be queried for the purpose of analysis.

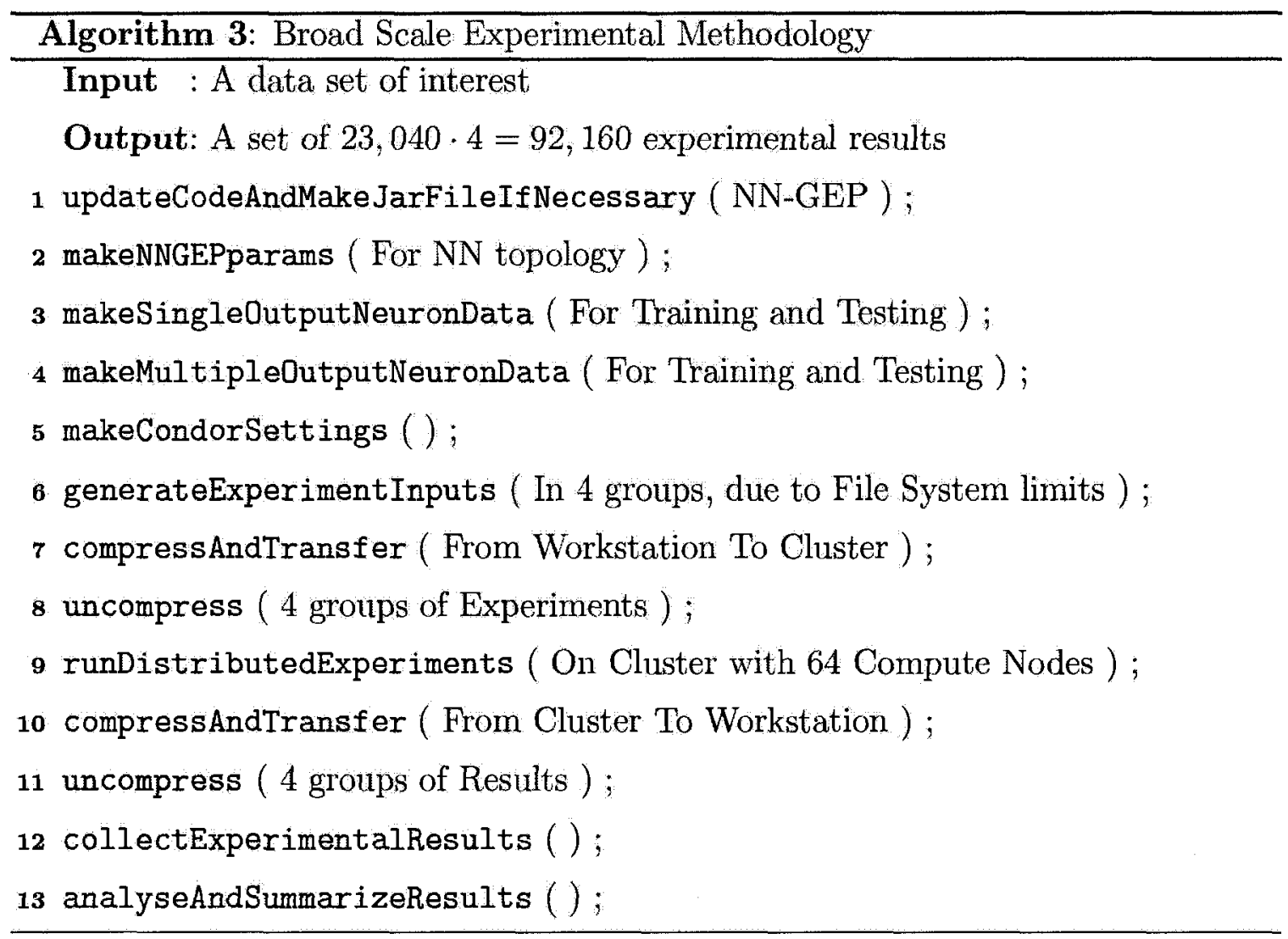

\subsubsection{Common Broad Scale Experimental Settings for NN-GEP}

The broad scale experimental settings are reported in Table 6.6 on page 104. They are grouped into 8 sets of 11,520 experiments, where 11 parameters are varied within each group. All other parameters are held constant. It is easy to see that there are a large number of parameters that may take on a large number of values and that this thesis only explores a small fraction; some related to the neural network (NN) and some related to the evolutionary computation algorithm (GEP).

\subsubsection{Selection Criteria for Broad Scale Experimental Settings}

Tradeoffs had to be made when selecting parameters for investigation (Table 6.6 on page 104). It was done in such a way as to try an make use of previous experience 
Table 6.6: Broad Scale Experimental Settings. There are 8 groups; indicated by $\square$. Within one group of 11,520 experiments, 11 variables are varied; indicated by $\checkmark$. Default values (See Section 2.2.4.2 on page 27) are marked as ${ }^{\text {def }}$. See Table 6.4 on page 101 for the published experimental settings.

\begin{tabular}{|c|c|}
\hline$\overline{\square \text { Error Measure }}$ & Classical $\left(\mathcal{E}_{C}\right)$ or Normalized $\left(\mathcal{E}_{C_{N}}\right)$ \\
\hline Neural Network Architecture & Number of layers and neurons/layer fixed \\
\hline NN Output Layer & Single or Multiple Neurons \\
\hline$\square$ NN Output Vector Mapping Function & ${ }_{f_{1}} \aleph_{1}^{\ell_{\text {out }},}, f_{2} \aleph_{1}^{\ell_{\text {out }}, f_{1}} \aleph_{>1}^{\ell_{\text {out }},{ }_{f_{2}}} \aleph_{>1}^{\ell_{\text {out }}}$ \\
\hline Tolerance for Mapping Function $f_{f_{2}} \aleph_{>1}^{\ell_{\text {out }}}$ & $\varepsilon=0.001^{\text {def }}$ \\
\hline$\checkmark N N$ Input Layer Constant Weights & 1, Number-Of-Input-Variables \\
\hline NN Input Layer Terminal Weights & 1 \\
\hline$\checkmark$ NN Hidden Layer Constant Weights & 1, Number-Of-Input-Variables \\
\hline NN Hidden Layer Terminal Weights & 1 \\
\hline NN Output Layer Constant Weights & 1 \\
\hline NN Output Layer Terminal Weights & 1 \\
\hline$\checkmark$ GEP Seeds & 5 unique (randomly chosen) seeds \\
\hline GEP Number Chromosomes/Individual & Number of Neurons in NN architecture \\
\hline GEP Number Symbol Sets & 3 (Determined by NN arch.: one/layer) \\
\hline GEP Symbol Sets: Functions (Weights) & $\begin{array}{l}"+"(1), "=(1), " * "(1) \text { (See Table B.1 on } \\
\text { page 181) }\end{array}$ \\
\hline \multirow{3}{*}{ GEP Symbol Sets: Terminals } & Input Layer uses Input Variables \\
\hline & Hidden Layer uses Input Layer Outputs \\
\hline & Output Layer uses Hidden Layer Outputs \\
\hline$\checkmark$ GEP Number Genes/Chromosome & 1,2 \\
\hline$\checkmark$ GEP Gene Headsize & 2,3 \\
\hline GEP Gene Linking Function & "+" - used when multiple genes \\
\hline$\checkmark$ GEP Number Floating Constants / Gene & 1,5 \\
\hline GEP Floating Constant Limits & {$[-10.0,10.0]$} \\
\hline GEP Max Number of Generations & 500 \\
\hline$\checkmark$ GEP Population Size & $5,20,50$ \\
\hline GEP Number of Elite Individuals & 1 \\
\hline GEP Inversion Rate & $0.1^{\text {def }}$ \\
\hline GEP Mutation Rate & $0.044^{\text {def }}$ \\
\hline GEP IS Transposition Rate & $0.1^{d e f}$ \\
\hline GEP RIS Transposition Rate & $0.1^{\text {def }}$ \\
\hline GEP One Point Recombination Rate & $0.3^{\text {def }}$ \\
\hline GEP Two Point Recombination Rate & $0.3^{\text {def }}$ \\
\hline GEP Gene Recombination Rate & $0.1^{\text {def }}$ \\
\hline GEP Gene Transposition Rate & $0.1^{\text {def }}$ \\
\hline$\checkmark$ GEP Species RNC Mutation Rate & $0.01^{d e f}, 0.03$ \\
\hline$\checkmark$ GEP Species DC Mutation Rate & $0.044^{\text {def }}, 0.01,0.03$ \\
\hline$\checkmark$ GEP Species DC Inversion Rate & $0.1^{d e f}, 0.3$ \\
\hline$\checkmark$ GEP Species DC IS Transposition Rate & $0.1^{d e f}, 0.3$ \\
\hline
\end{tabular}


with the particular data sets and to focus on parameters that were felt to be more strongly related to good solutions. This approach is clearly heuristic in nature due to the fact that it is not feasible to explore the parameter space exhaustively. Networks with multiple output neurons, as in the classical case, and networks with single output neurons were investigated.

\subsubsection{Computation within One Broad Scale Experiment}

The way in which fitness is computed was previously described (Section 4.3 on page 72). In particular, 4 errors are computed $\left(\mathcal{E}_{C}^{T r}, \mathcal{E}_{C}^{T e}, \mathcal{E}_{C_{N}}^{T r}\right.$ and $\left.\mathcal{E}_{C_{N}}^{T e}\right)$ on both the training and testing data for each experiment in order to allow a direct comparison of all measures within the same resulting experimental database (see Appendix $\mathbf{C}$ on page 186). Only one of the training error measures $\left(\mathcal{E}_{C}^{T r}\right.$ or $\left.\mathcal{E}_{C_{N}}^{T r}\right)$ is used within the fitness of an individual (see Eq. 4.1 on page 73), and hence only one error measure is used for the optimization process. Evolution stops if the number of generations has been exhausted or a heuristic termination criteria has been met (see Section 4.5 on page 76). In addition, the neural network architecture affects the results (see Section 4.2.5 on page 66). This thesis computes the following network output vector mappings within the experiments: ${ }_{f_{1}} \aleph_{1}^{\ell_{\text {out }}},{ }_{f_{2}} \aleph_{1}^{\ell_{\text {out }}},{ }_{f_{1}} \aleph_{>1}^{\ell_{\text {out }}}$ and $f_{2} \aleph_{>1}^{\ell_{\text {out }}}$.

\subsubsection{Computation Time}

This thesis does not focus on constructing an algorithm that is optimal in terms of computation time (i.e. economy of computation). The easiest approach to reduce the current implementation's computation time is to only compute one neural network output mapping function within one experiment (See Section 6.2.3 on page 105). The main focus of this research has been to demonstrate that an alternative approach exists that is capable of the construction of a feed forward neural network as applied to real world data sets. The following subsections report to what degree this thesis has considered computation time. 


\subsubsection{Estimated Time Required for MRS}

The broad scale approach using MRS data was estimated to require $\approx 126.25$ years on a single CPU. Hence, the publicly available Breast Cancer Data was substituted (See Section 6.1.2 on page 94). The time estimate is: 4 output vector mapping functions times 11,520 experiments times 12 hours per experiment, yielding 552,960 hours on $1 \mathrm{CPU}$, which is 23,040 days or 63.12 years per mapping function. Since this thesis investigates 2 mapping functions within a broad scale experiment per data set, the total estimated time for completion of a MRS investigation on a single CPU is $63.12 \cdot 2=126.25$ years.

\subsubsection{Substrate for Aiding Time Reduction}

Using one computer is not feasible for this research (See Section 6.2.4.1 on page 106). Instead a distributed computing approach was taken using Condor and the NRCIIT-KD's cluster ${ }^{1}$ of 64 compute nodes. This allowed, for example, the Werenskiold experiments $((\operatorname{card}($ Exps $)=92,160))$ to be computed within $\approx 4$ days instead of $\approx 4 \cdot 64=\approx 256$ days on a single CPU. But the problem compounds, because 3 data sets were investigated within a broad scale search $((\operatorname{card}($ Exps $)=276,480))$ leading to $\approx 4 \cdot 3=\approx 12$ days of compute time on the cluster instead of $\approx 256 \cdot 3=\approx 768$ days or $\approx 2.1$ years of compute time on a single CPU. In addition the MR spectra were investigated adding even more to the amount of computing resources required (See Section 6.2.4.1 on page 106).

To aid such a broad investigation, the Condor submission program, which was also automatically generated (see Appendix A on page 175), is used to specify all of the data and configuration files for the programs that will execute on the remote host. The submission process enables Condor to:

i) schedule jobs for execution

ii) check point them (put a job on hold)

iii) transfer all data to the remote host

\footnotetext{
${ }^{1}$ NRC - National Research Council, JJT - Institute for Information Technology, KD - Knowledge Discovery Group
} 
iv) transfer all generated data back to the local host (submitting machine)

\subsubsection{Policy for Selection of the Best NN-GEP Solutions}

The broad scale experiments lead to ( $\operatorname{card}($ Exps $)=92,160)$ experimental results (i.e. neural networks). It is not possible to report all of them within this thesis, so a subset have to be selected. All of the results for a particular data set have been partitioned into solutions referred to as good, ok and bad when analyzing the respective parameter spaces (See Section 7.2 on page 134). When reporting a specific neural network solution as it's associated analytic function (See Section 7.1 on page 109), the general strategy is to pick a neural network that has gond training and testing performance, while maintaining a simple associated analytic function. Simple is a subjective term. In this thesis, a simple analytic function means that the analytic function uses as few input variables $\left(v_{1}, v_{2}, \cdots\right)$ and as few primitive functions (e.g. + ) as possible.

For the selected results reported in Section 7.1.1.1 on page 115 and Section 7.1.2.1 on page 121, the strategy for each of the 8 groups of experiments is to:

1. open the query containing the $(\operatorname{card}(\mathrm{Exps})=11,520)$ results (See Appendix $\mathbf{C}$ on page 186)

2. sum the training and testing errors $\left(\mathcal{E}_{C}^{T r}+\mathcal{E}_{C}^{T e}\right)$ or $\left(\mathcal{E}_{C_{N}}^{T r}+\mathcal{E}_{C_{N}}^{T e}\right)$ for the particular neural network output mapping function (See Section 4.2.5 on page 66) under consideration $\left({ }_{f_{1}} \aleph_{1}^{\ell_{\text {out }},}, f_{2} \aleph_{1}^{\ell_{\text {out }},}{ }_{f_{1}} \aleph_{>1}^{\ell_{\text {out }}}\right.$, or $\left.{ }_{f_{2}} \aleph_{>1}^{\ell_{\text {out }}}\right)$

3. sort by the sum into ascending order

4. select a solution within the top 160 results (i.e. within the 160 smallest sums) that has a simple analytic equation associated to the neurons in the output layer

For the selected results reported in Section 7.1.3.1 on page 128, the strategy was different. Not all of the 8 groups of experiments produced satisfactory error values. Therefore, only the results associated with ${ }_{f_{1}} \aleph_{>1}^{\ell_{\text {out }}}$ and with $\mathcal{E}_{C}$ were selected. The reason is due to the ranking as reported in Table 7.1 on page 110. In addition, for the discussion in Section 7.2 on page 134 it can be seen that Table 7.7 on page 140 contains good networks associated to ${ }_{f_{1}} \aleph_{>1}^{\ell_{\text {out }}}$ most frequently $(1,794$ and 1,059$)$. 


\subsection{Chapter Summary}

This chapter has described each of the 4 data sets that are used within this thesis. In addition, the experimental methodology has been presented in which a parsimonious approach to experimental design has been taken, namely, uniformly picking values for 11 controlling algorithm parameters for each of 8 groups. Both single output neuron and multiple output neuron neural networks are of interest within this heuristic investigation. But how do they actually perform? That is what shall be reported next. 


\section{Chapter 7}

\section{Experimental Results}

Everyone thinks that the data in other people's subjects are in better shape.

John Wilder Tukey, 1915-2000

The previous chapter detailed all of the experimental settings for which this chapter now reports. Results for both the broad scale experiments and for the published experiments are described. For the broad scale experiments all 3 data set results are reported. A summary of all of these results is presented in Table 7.1 on page 110 . Further, a discussion of the parameter space for one series of experiments using $f_{1} \aleph_{>1}^{\ell_{\text {out }}}$ for each of the 3 data sets is described in Section $\mathbf{7 . 2}$ on page 134. A series of comparisons with other techniques is performed. The chapter then concludes with published results of applying NN-GEP to Magnetic Resonance Data.

\subsection{Broad Scale Experimental Results}

A series of broad scale experiments using the settings in Table 6.6 on page 104 were performed on 3 data sets: i) Insunza, ii) Public Breast Cancer, iii) Werenskiold. Table 7.1 on page 110 reports 4 averages (2 error averages $\overline{\mathcal{E}^{T r}}, \overline{\mathcal{E}^{T e}}$, and 2 ranked error averages $\overline{\mathcal{E}_{\mathcal{R}}^{T r}}, \overline{\mathcal{E}_{\mathcal{R}}^{T e}}$ ) for each of the 4 network output vector mapping functions: $f_{1} \aleph_{1}^{\ell_{\text {out }}, f_{2}} \aleph_{1}^{\ell_{\text {out }},{ }_{f_{1}}} \aleph_{>1}^{\ell_{\text {out }},}{ }_{f_{2}} \aleph_{>1}^{\ell_{\text {out }}}$, and for the 2 error measures: $\mathcal{E}_{C}$, and $\mathcal{E}_{C_{N}}$ (See Section 4.3.1 on page 73) that were investigated. It can be seen that based only on Table 7.1 on page 110, the mapping functions performing the best and ordered down

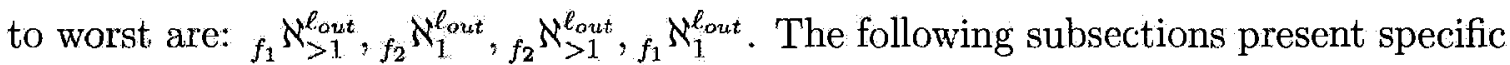
results for each of the data sets; such as the error distributions for each of the 4 mapping functions for each of the 2 error measures, and a comparison of all pairs of distributions over the training and testing data sets. In addition, a later section (Section 7.2 on page 134) discusses the controlling algorithm parameters that were investigated within the broad scale experiments. 
Table 7.1: Broad scale results summary; including rankings for each mapping function over all $\mathcal{E}$ and data sets.

\begin{tabular}{|c|c|c|c|c|c|c|c|c|c|}
\hline \multirow[b]{3}{*}{ Input Data } & \multirow{3}{*}{ Minimize } & \multicolumn{4}{|c|}{ One Output Neuron } & \multicolumn{4}{|c|}{ Multiple Output Neurons } \\
\hline & & \multicolumn{2}{|c|}{$f_{1} \aleph_{1}^{\ell_{\text {out }}}(x)$} & \multicolumn{2}{|c|}{${ }_{f_{2}} \aleph_{1}^{\ell_{\text {out }}}(x)$} & \multicolumn{2}{|c|}{$f_{1} \aleph_{>1}^{\ell_{\text {ont }}}(x)$} & \multicolumn{2}{|c|}{$f_{2} \aleph_{>1}^{\ell_{\text {out }}}(x)$} \\
\hline & & $\overline{\mathcal{E}^{T r}, \overline{\mathcal{E}_{\mathcal{R}}^{T r}}}$ & $\overline{\mathcal{E}^{T e}}, \overline{\mathcal{E}_{\mathcal{R}}^{T e}}$ & $\overline{\mathcal{E}^{T r}}, \overline{\mathcal{E}_{\mathcal{R}}^{T r}}$ & $\overline{\mathcal{E}^{T e}}, \overline{\mathcal{E}_{\mathcal{R}}^{T e}}$ & $\overline{\mathcal{E}^{T r}}, \overline{\mathcal{E}_{\mathcal{R}}^{T r}}$ & $\overline{\mathcal{E}^{T e}}, \overline{\mathcal{E}_{\mathcal{R}}^{T e}}$ & $\overline{\mathcal{E}^{T r}}, \overline{\mathcal{E}_{\mathcal{R}}^{T r}}$ & $\overline{\mathcal{E}^{T e}}, \overline{\mathcal{E}_{\mathcal{R}}^{T_{e}}}$ \\
\hline \multicolumn{2}{|l|}{ Insunza } & \multicolumn{4}{|c|}{$5-1-1$} & \multicolumn{4}{|c|}{$5-1-2$} \\
\hline \multirow[t]{2}{*}{$(c=1)$} & $\mathcal{E}_{C}$ & $0.130,3.53$ & $0.128,3.39$ & $0.083,1.92$ & $0.075,1.94$ & $0.080,1.90$ & $0.073,1.92$ & $0.113,2.646$ & $0.107,2.75$ \\
\hline & $\mathcal{E}_{C_{N}}$ & $0.478,3.52$ & $0.477,3.35$ & $0.329,1.91$ & $0.319,1.94$ & $0.328,1.89$ & $0.317,1.93$ & $0.410,2.682$ & $0.418,2.78$ \\
\hline \multicolumn{2}{|c|}{ Breast Cancer } & \multicolumn{4}{|c|}{$9-2-1$} & \multicolumn{4}{|c|}{$9-2-2$} \\
\hline \multirow[t]{2}{*}{$(c=2)$} & $\mathcal{E}_{C}$ & $0.181,3.38$ & $0.126,3.40$ & $0.077,1.87$ & $0.040,1.90$ & $0.082,1.95$ & $0.044,1.941$ & $0.151,2.791$ & $0.107,2.76$ \\
\hline & $\mathcal{E}_{C_{N}}$ & $0.216,3.39$ & $0.168,3.42$ & $0.085,1.87$ & $0.046,1.89$ & $0.092,1.95$ & $0.052,1.93$ & $0.178,2.79$ & $0.130,2.76$ \\
\hline \multicolumn{2}{|l|}{ Werenskiold } & \multicolumn{4}{|c|}{$9-2-1$} & \multicolumn{4}{|c|}{$9-2-5$} \\
\hline \multirow[t]{2}{*}{$(c=5)$} & $\mathcal{E}_{C}$ & $0.688,3.49$ & $0.659,3.37$ & $0.576,1.88$ & $0.549,1.98$ & $0.559,1.80$ & $0.524,1.76$ & $0.644,2.83$ & $0.619,2.89$ \\
\hline & $\mathcal{E}_{C_{N}}$ & $0.740,2.92$ & $0.726,2.83$ & $0.670,2.67$ & $0.703,2.38$ & $0.660,1.88$ & $0.652,1.83$ & $0.740,2.93$ & $0.743,2.96$ \\
\hline \multirow[t]{3}{*}{ Average $\mathcal{R}$} & $\mathcal{E}_{C}$ & 3.47 & 3.39 & 1.89 & 1.94 & 1.88 & 1.87 & 2.76 & 2.8 \\
\hline & $\mathcal{E}_{C_{N}}$ & 3.28 & 3.2 & 2.15 & 2.07 & 1.91 & 1.90 & 2.80 & 2.83 \\
\hline & Average & 3.375 & 3.295 & 2.02 & 2.005 & 1.895 & 1.885 & 2.78 & 2.81 .5 \\
\hline $\begin{array}{l}\text { Overall } \\
\text { Ranking }\end{array}$ & $\begin{array}{l}\text { Training } \\
\text { Testing }\end{array}$ & 4 & 4 & 2 & 2 & 1 & 1 & 3 & 3 \\
\hline
\end{tabular}




\subsubsection{Insunza Broad Scale Results}

The Insunza error distributions for all mapping functions are reported in Fig. 7.1 on page 113 and Fig. 7.2 on page 114. The training error distributions are reported in the left column, while the right columm contains the testing error distributions. The center column contains a training error versus testing error plot in order to visualize the differences more clearly. The center column also has a $y=x$ line drawn to give an indication of how well the testing data performs with respect to the training data (above/below line).

In general, Fig. 7.1 on page 113 has very highly skewed distributions towards lower error values for both training and testing, while Fig. 7.2 on page 114 contains

a much broader range of neural network solution errors. In fact, $\mathcal{E}_{C_{N}}^{T r}\left(f_{1} \aleph_{1}^{\ell_{\text {out }}}\right)$ seems to be a very poor result because it is skewed towards high error values for both training and testing. Luckily, $\mathcal{E}_{C}^{T r}\left({ }_{f_{1}} \aleph_{>1}^{\ell_{\text {out }}}\right)$ has a very nice error distribution in terms of both skewness and low absolute error values.

A visual inspection for the differences in the training and testing error distributions may be done by comparing the relevant graphs within Fig. 7.1 on page 113 and Fig. 7.2 on page 114. However, Table 7.2 on page 112 performs a pairwise comparison for all training error and all testing error distributions and reports the Komolgorov-Smirnov D statistic (See Section 2.3.3 on page 37). A small value indicates that the two error distributions are quite similar, and that they may, in fact, be from the same underlying distribution. Put another way, large D values indicate departure from the null hypothesis that the two distributions do not differ. For example, the $\mathrm{D}$ statistic value when comparing the training error distributions for $\mathcal{E}_{C}\left({ }_{f_{2}} \aleph_{1}^{\ell_{\text {out }}}\right)$ and $\mathcal{E}_{C}\left({ }_{f_{1}} \aleph_{>1}^{\ell_{\text {out }}}\right)$ is 1.27 indicating that the distributions may come from the same underlying distribution. It is also interesting that the $\mathrm{D}$ statistic for the testing distributions for the same 2 error measures is slightly higher at 1.75, which is a good confirmation that it is quite likely that these two error measures have the same underlying distribution. 
Table 7.2: Pairwise comparison of Insunza error distributions. Komolgorov-Smirnov D Statistic and associated hypothesis testing probability (See Section 2.3.3 on page 37) for Pairwise Comparison of Training (upper triangular matrix) and Testing (lower triangular matrix) for two Error Distributions $\left(\mathcal{E}_{C}, \mathcal{E}_{C_{N}}\right)$. Large D values indicate the degree of difference in the two distributions. Small probability values show that the CDF of the first error data set is significantly different than the second error data set. Each distribution contains 11,520 FFNN solution errors.

Komolgorov-Smirnov D Statistic

\begin{tabular}{|c|c|c|c|c|}
\hline & $\mathcal{E}_{C}\left({ }_{f} \aleph_{1}^{\ell_{\text {out }}}\right)$ & $\mathcal{E}_{C}\left({ }_{f_{2}} \aleph_{1}^{\ell_{\text {out }}}\right)$ & $\mathcal{E}_{C}\left({ }_{f_{1}} \aleph_{>1}^{\ell_{\text {out }}}\right)$ & $\mathcal{E}_{C}\left({ }_{f_{2}} \aleph_{>1}^{\ell_{\text {out }}}\right)$ \\
\hline $\mathcal{E}_{C}\left(f_{1} \aleph_{1}^{\ell_{\text {out }}}\right)$ & & 51.83 & 52.76 & 27.14 \\
\hline $\mathcal{E}_{C}\left({ }_{f_{2}} \aleph_{1}^{\ell_{o u t}}\right)$ & 56.33 & & 1.27 & 25,56 \\
\hline $\mathcal{E}_{C}\left({ }_{f_{1}} N_{>1} \bar{\ell}_{\text {out }}\right)$ & 57.72 & 1.75 & & 26.35 \\
\hline $\mathcal{E}_{C}\left({ }_{f_{2}} \aleph_{>1}^{\ell_{\text {out }}}\right)$ & 39.37 & 20.05 & 21.06 & \\
\hline
\end{tabular}

\begin{tabular}{|c|c|c|c|c|}
\hline & $\mathcal{E}_{C_{N}}\left(f_{1} \aleph_{1}^{\ell_{\text {out }}}\right)$ & $\mathcal{E}_{C_{N}}\left({ }_{f_{2}} \aleph_{1}^{\left.\ell_{\text {out }}\right)}\right.$ & $\mathcal{E}_{C_{N}}\left({ }_{f_{1}} \aleph_{>1}^{\ell_{\text {out }}}\right)$ & $\mathcal{E}_{C_{N}}\left({ }_{f_{2}} \aleph_{>1}^{\left.\ell_{\text {out }}\right)}\right.$ \\
\hline$\overline{\mathcal{E}_{C_{N}}\left({ }_{f_{1}} \aleph_{1}^{\ell_{\text {out }}}\right)}$ & & 49.79 & 50.24 & 24.80 \\
\hline $\mathcal{E}_{C_{N}}\left({ }_{f_{2}} \aleph_{1}^{\ell_{\text {out }}}\right)$ & 53.72 & & 0.88 & 26.43 \\
\hline $\mathcal{E}_{C_{N}}\left({ }_{f_{1}} \aleph_{>1}^{\ell_{\text {out }}}\right)$ & 54.63 & 1.52 & & 26.61 \\
\hline $\mathcal{E}_{C_{N}}\left(f_{2} N_{>1}^{\ell_{\text {out }}}\right)$ & 36.13 & 21.89 & 22.22 & \\
\hline
\end{tabular}

Komolgorov-Smirnov Probability

\begin{tabular}{l||c|ccc} 
& $\mathcal{E}_{C}\left({ }_{f_{1}} \aleph_{1}^{\ell_{\text {out }}}\right)$ & $\mathcal{E}_{C}\left(f_{2} \aleph_{1}^{\ell_{\text {out }}}\right)$ & $\mathcal{E}_{C}\left({ }_{f_{1}} \aleph_{>1}^{\ell_{\text {out }}}\right)$ & $\mathcal{E}_{C}\left({ }_{f_{2}} \aleph_{>1}^{\ell_{\text {out }}}\right)$ \\
\hline $\mathcal{E}_{C}\left({ }_{f_{1}} \aleph_{1}^{\ell_{\text {out }}}\right)$ & & 0.00 & 0.00 & 0.00 \\
$\mathcal{E}_{C}\left({ }_{f_{2}} \aleph_{1}^{\ell_{\text {out }}}\right)$ & 0.00 & & 0.08 & 0.00 \\
$\mathcal{E}_{C}\left({ }_{f_{1}} \aleph_{>1}^{\ell_{\text {out }}}\right)$ & 0.00 & 0.00 & & 0.00 \\
$\mathcal{E}_{C}\left({ }_{f_{2}} \aleph_{>1}^{\mathcal{E}_{\text {out }}}\right)$ & 0.00 & 0.00 & 0.00 &
\end{tabular}

\begin{tabular}{|c|c|c|c|c|}
\hline & $\mathcal{E}_{C_{N}}\left({ }_{f_{1}} \aleph_{1}^{\ell_{\text {out }}}\right)$ & $\mathcal{E}_{O_{N}}\left({ }_{f_{2}} N_{1}^{f_{\text {out }}}\right)$ & $\mathcal{E}_{C_{N}}\left({ }_{f_{1}} \aleph_{>1}^{\ell_{\text {out }}}\right)$ & $\mathcal{E}_{C_{N}}\left({ }_{f_{2}} N_{>1}^{\ell_{\text {out }}}\right)$ \\
\hline$\overline{\mathcal{E}_{C_{N}}\left(f_{1} N_{1}^{\ell_{\text {out }}}\right)}$ & & 0.00 & 0.00 & 0.00 \\
\hline $\mathcal{E}_{C_{N}}\left(f_{2} \aleph_{1}^{\ell_{\text {out }}}\right)$ & 0.00 & & 0.42 & 0.00 \\
\hline $\mathcal{E}_{C_{N}}\left({ }_{f} \aleph^{\ell_{o u t}}\right)$ & 0.00 & 0.02 & & 0.00 \\
\hline $\mathcal{E}_{C_{N}}\left({ }_{f_{2}} \aleph_{>1}^{\ell_{\text {out }}}\right)$ & 0.00 & 0.00 & 0.00 & \\
\hline
\end{tabular}




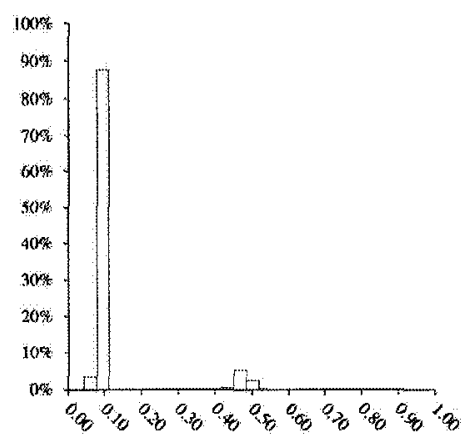

(a) $\mathcal{E}_{C}^{T r}\left({ }_{f_{1}} \aleph_{1}^{\ell_{\text {out }}}\right), \mathrm{NN}: 5-1-1$

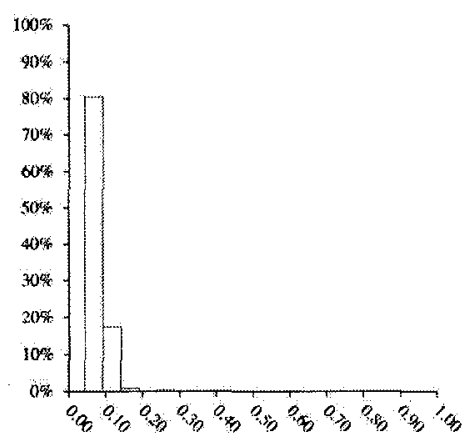

(d) $\mathcal{E}_{C}^{T r}\left({ }_{f_{2}} \aleph_{1}^{\ell_{\text {cut }}}\right), \mathrm{NN}: 5-1-1$

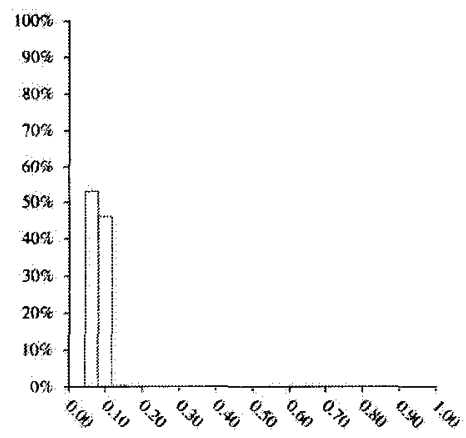

(g) $\mathcal{E}_{C}^{\mathrm{Tr}}\left({ }_{f_{1}} \aleph_{>1}^{\ell_{\text {out }}}\right), \mathrm{NN}: 5-1-2$

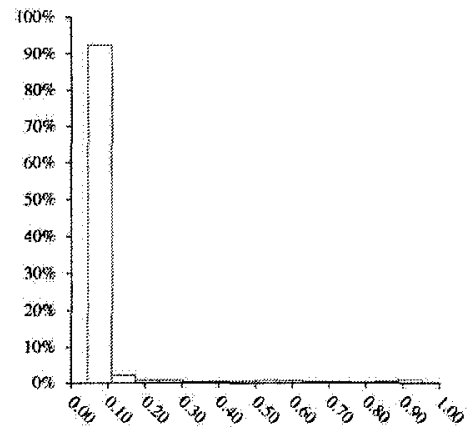

(j) $\mathcal{E}_{C}^{T r}\left({ }_{f_{2}} \aleph_{>1}^{\ell_{\text {out }}}\right), \mathrm{NN}: 5-1-2$

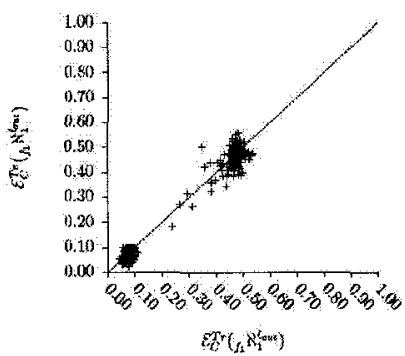

(b) Training vs. Testing

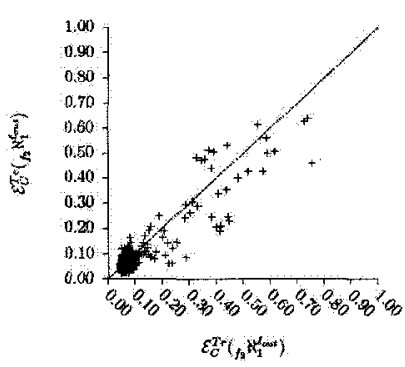

(e) Training vs. Testing

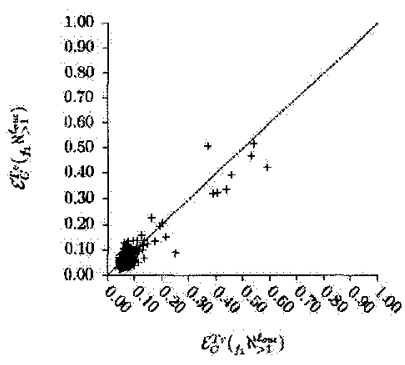

(h) Training vs. Testing

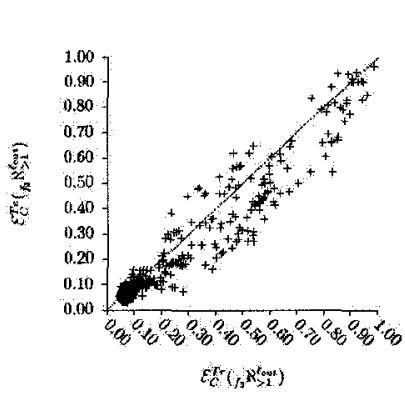

(k) Training vs. Testing

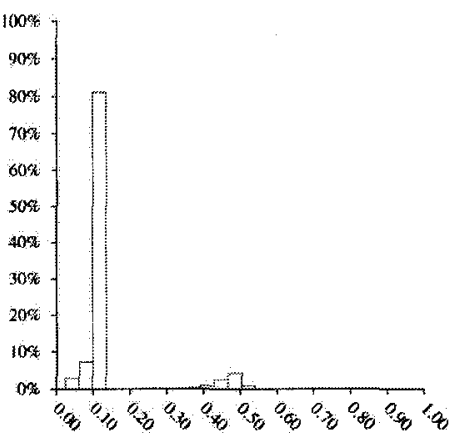

(c) $\mathcal{E}_{C}^{T e}\left({ }_{f_{1}} \aleph_{1}^{\ell_{\text {out }}}\right)$

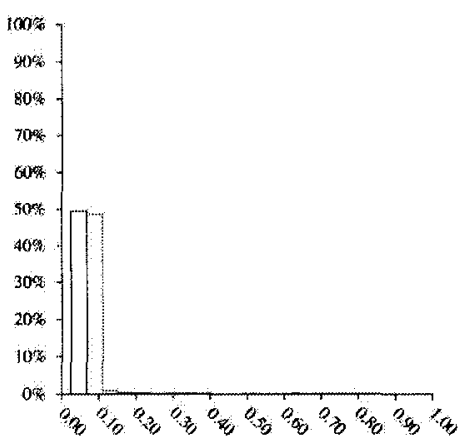

(f) $\mathcal{E}_{C}^{T e}\left({ }_{f_{2}} \aleph_{1}^{\ell \text { out }}\right)$

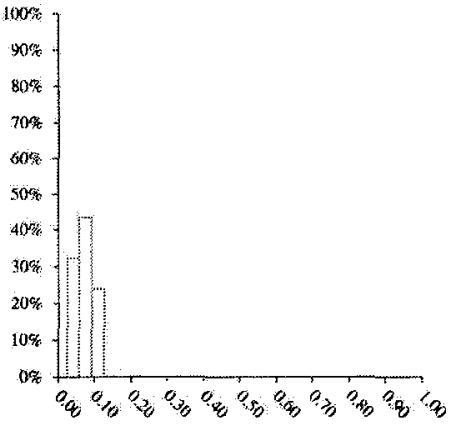

(i) $\mathcal{E}_{C}^{T^{\top} e}\left(f_{1} \aleph_{>1}^{l_{\text {out }} t}\right)$

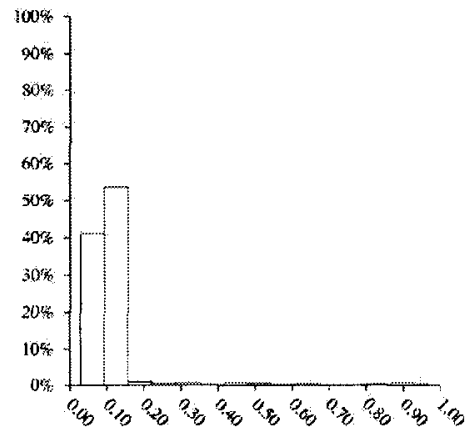

(l) $\mathcal{E}_{C}^{T e}\left(f_{2} \aleph_{>1}^{\ell_{\text {out }}}\right)$

Figure 7.1: Insunza $\left(\mathcal{E}_{C}\right)$. $(\operatorname{card}(\operatorname{Exps})=11,520)$. Sturges binning. 


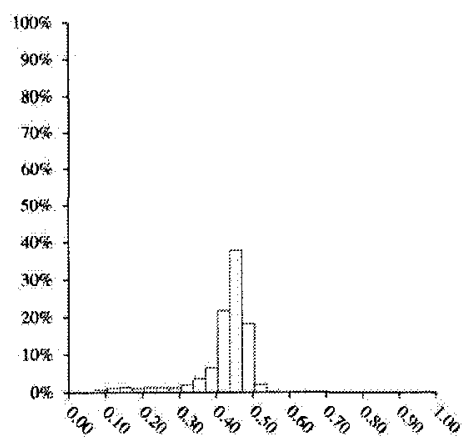

(a) $\mathcal{E}_{C_{N}}^{T r}\left({ }_{f_{1}} \aleph_{1}^{\ell_{\text {out }}}\right), \mathrm{NN}: 5-1-1$

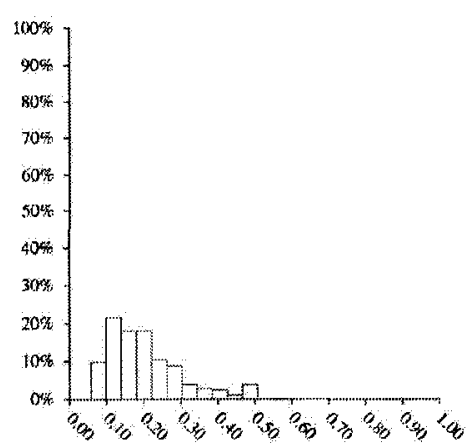

(d) $\mathcal{E}_{C_{N}}^{T r}\left(f_{2} \aleph_{1}^{\ell_{\text {out }}}\right)$, NN:5-1-1

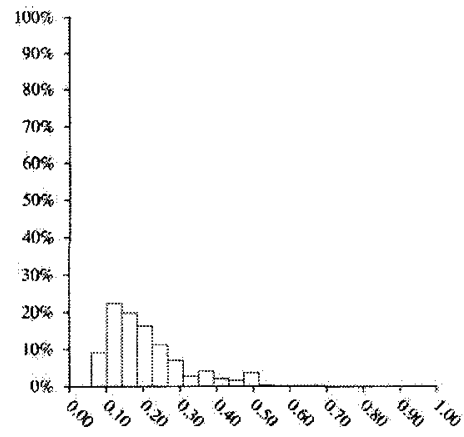

(g) $\mathcal{E}_{C_{N}}^{T r}\left({ }_{f i} \aleph_{>1}^{\ell_{\text {out }}}\right)$, NN:5-1-2

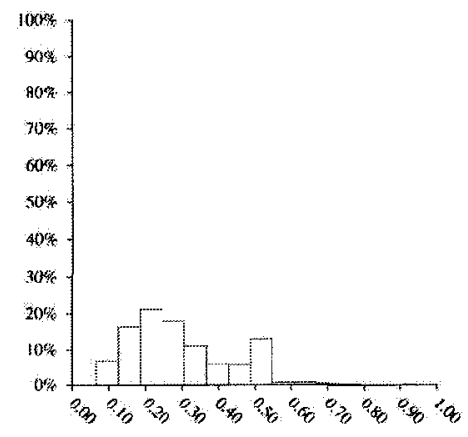

(j) $\mathcal{E}_{C_{N}}^{T r}\left({ }_{f_{2}} \aleph_{>1}^{\ell_{\text {out }}}\right)$, NN:5-1-2

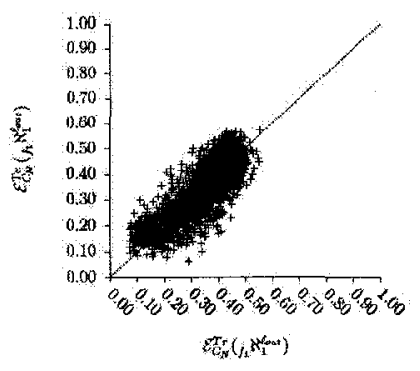

(b) Training vs. Testing

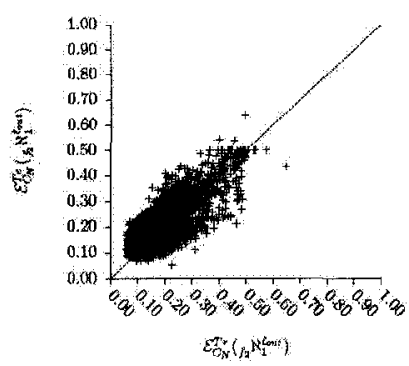

(e) Training vs. Testing

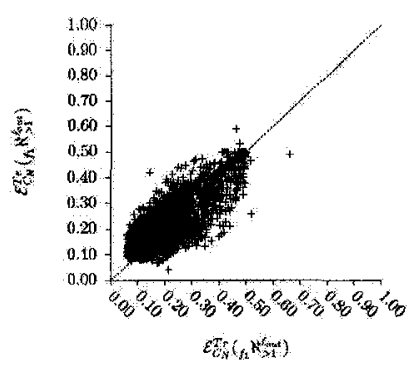

(h) Training vs. Testing

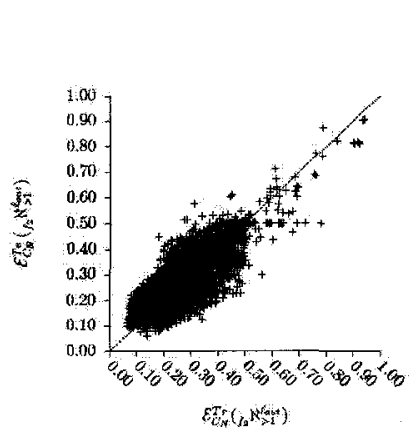

(k) Training vs. Testing

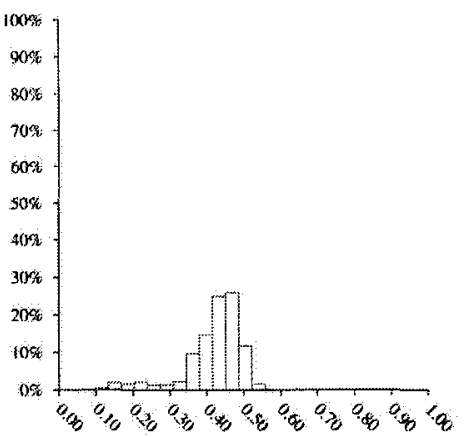

(c) $\mathcal{E}_{C_{N}}^{T e}\left(f_{1} \aleph_{1}^{\ell_{\text {out }}}\right)$

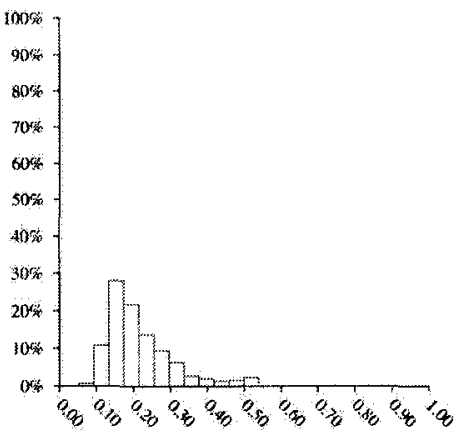

(f) $\mathcal{E}_{C_{N}}^{T_{N}^{e}}\left(f_{2} \aleph_{1}^{\ell_{\text {out }}}\right)$

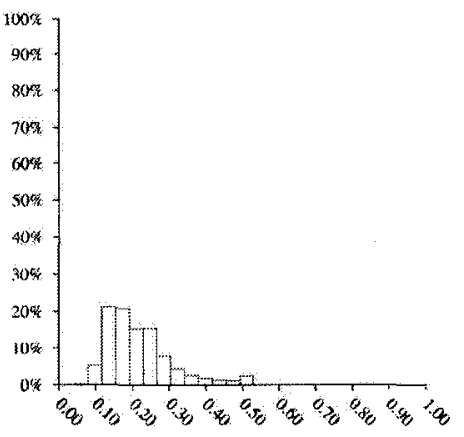

(i) $\mathcal{E}_{C_{N}}^{T e}\left(f_{1} \aleph_{>1}^{\ell_{\text {out }}}\right)$

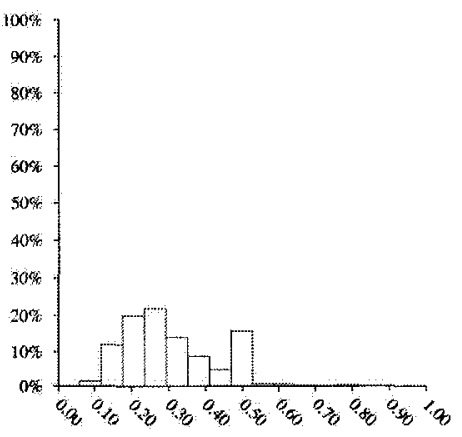

(l) $\mathcal{E}_{\mathrm{C}_{N}}^{T e}\left(f_{2} \aleph_{>1}^{\ell_{\text {out }}}\right)$

Figure 7.2: Insunza $\left(\mathcal{E}_{C_{N}}\right)$. (card $\left.(\operatorname{Exps})=11,520\right)$. Sturges binning. 


\subsubsection{Selected Insunza Results}

Not all $(\operatorname{card}(\mathrm{Exps})=92,160)$ experimental results may be reported, so a subset have been chosen for the Insunza investigation (See Section 6.2.5 on page 107).

The Insunza learning problem is related to spatial locations of underground caves. Fig. 7.3 on page 118 and Fig. 7.4 on page 119 show the maps (spatial representation of the associated equations) for the following equation results.

For network output vector mapping function ${ }_{f_{1}} \aleph_{1}^{\ell_{\text {out }}}$, experiment $\operatorname{Exp}_{11,505}^{\mathcal{E}_{C}, \mathrm{NN}: 5-1-1}$ yields the following solution that results in the image in Fig. 7.3 on page 118(a):

$$
\begin{aligned}
& \mathcal{E}_{C}^{T r}=0.052: c_{1}=\frac{855}{884}, c_{2}=\frac{75}{97} \\
& \mathcal{E}_{C}^{T e}=0.033: c_{1}=\frac{217}{220}, c_{2}=\frac{19}{24}
\end{aligned}
$$

Output Neuron Functions expanded to contain input variables:

$$
0.02\left(v_{1}+2 \cdot v_{2}+v_{3}+v_{5}\right)-5.66
$$

For network output vector mapping function ${ }_{f_{2}} \aleph_{1}^{\ell_{\text {out }}}$, experiment yields the following solutions that results in solution 1 being reported in Fig. 7.3 on page 118(c) (See Section 3.5 on page 56 ):

$$
\begin{aligned}
& \mathcal{E}_{C}^{T r}=0.054: c_{1}=\frac{855}{884}, c_{2}=\frac{73}{97} \\
& \mathcal{E}_{C}^{T e}=0.033: c_{1}=\frac{217}{220}, c_{2}=\frac{19}{24}
\end{aligned}
$$

Output Neuron Functions expanded to contain input variables:

1. Solution $1 \operatorname{Exp}_{18,466}^{\mathcal{E}_{C}, \mathrm{NN}: 5-1-1}$ :

$$
\left((-4.61)-\left(\left(\left(\left(\left(v_{1}+v_{2}\right)+v_{2}\right)\right)+\left(\left(v_{5}+v_{3}\right)\right)\right)\right)\right)
$$

2. Solution $2 \operatorname{Exp}_{18,490}^{\mathcal{E}_{\mathcal{C}, N N}: 5-1-1}$ :

$$
\left((-4.61)-\left(\left(\left(\left(\left(v_{5}+v_{2}\right)+v_{2}\right)\right)+\left(\left(v_{1}+v_{3}\right)\right)\right)\right)\right)
$$

3. Both Solution 1 and Solution 2 simplify to:

$$
-v_{1}-2 \cdot v_{2}-v_{3}-v_{5}-4.61
$$


For network output vector mapping function ${ }_{f_{1}} \aleph_{>1}^{\ell_{\text {out }}}$, experiment $\operatorname{Exp}_{12,561}^{\mathcal{E}_{C}, \mathrm{NN}: 5-1-2}$ yields the following solution that results in the image in Fig. 7.3 on page 118(b):

$$
\begin{aligned}
& \mathcal{E}_{C}^{T r}=0.052: c_{1}=\frac{857}{884}, c_{2}=\frac{73}{97} \\
& \mathcal{E}_{C}^{T e}=0.033: c_{1}=\frac{217}{220}, c_{2}=\frac{19}{24}
\end{aligned}
$$

Output Neuron Functions expanded to contain input variables:

$$
-v_{1} \cdot v_{2}+v_{2}+v_{3}+v_{5}+3.56
$$

For network output vector mapping function $f_{2} \aleph_{>1}^{\ell_{\text {out }}}$, experiment $\operatorname{Exp}_{9,498}^{\mathcal{E}_{C}, \mathrm{NN}: 5-1-2}$ yields the following solution that results in the image in Fig. 7.3 on page 118(d):

$$
\begin{aligned}
& \mathcal{E}_{C}^{T r}=0.062: c_{1}=\frac{841}{884}, c_{2}=\frac{79}{97} \\
& \mathcal{E}_{C}^{T e}=0.045: c_{1}=\frac{215}{220}, c_{2}=\frac{18}{24}
\end{aligned}
$$

Output Neuron Functions expanded to contain input variables:

$$
\begin{array}{r}
-56.64 \cdot\left(-2 \cdot v_{1}-v_{2}-v_{3}-2 \cdot v_{5}-5.90\right) \\
35.54 \cdot\left(-2 \cdot v_{1}-v_{2}-v_{3}-2 \cdot v_{5}-5.90\right)
\end{array}
$$

For network output vector mapping function ${ }_{f_{1}} \aleph_{1}^{\ell_{\text {out }}}$, experiment $\operatorname{Exp}_{21,769}^{\mathcal{E}_{C_{N}}, \mathrm{NN}: 5-1-1}$ yields the following solution that results in the image in Fig. 7.4 on page 119(a):

$$
\begin{aligned}
& \mathcal{E}_{C_{N}}^{T r}=0.095: c_{1}=\frac{780}{884}, c_{2}=\frac{90}{97} \\
& \mathcal{E}_{C_{N}}^{T e}=0.140: c_{1}=\frac{195}{220}, c_{2}=\frac{20}{24}
\end{aligned}
$$

Output Neuron Functions expanded to contain input variables:

$$
0.061 \cdot\left(v_{2}+v_{3}+v_{4}\right)-5.66
$$

For network output vector mapping function ${ }_{f_{2}} \aleph_{1}^{\ell_{\text {out }}}$, experiment $\operatorname{Exp}_{16,138} \mathcal{E}_{C_{N}, N N: 5-1-1}$ yields the following solution that results in the image in Fig. 7.4 on page 119(c): 


$$
\begin{aligned}
& \mathcal{E}_{C_{N}}^{T r}=0.077: c_{1}=\frac{803}{884}, c_{2}=\frac{91}{97} \\
& \mathcal{E}_{C_{N}}^{T e}=0.091: c_{1}=\frac{189}{220}, c_{2}=\frac{23}{24}
\end{aligned}
$$

Output Neuron Functions expanded to contain input variables:

$$
-v_{2}-v_{3}-v_{4}-v_{5}-2.02
$$

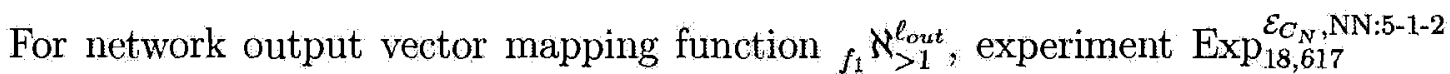
yields the following solution that results in the image in Fig. 7.4 on page 119(b):

$$
\begin{aligned}
& \mathcal{E}_{C_{N}}^{T r}=0.076: c_{1}=\frac{813}{884}, c_{2}=\frac{90}{97} \\
& \mathcal{E}_{C_{N}}^{T e}=0.092: c_{1}=\frac{198}{220}, c_{2}=\frac{22}{24}
\end{aligned}
$$

Output Neuron Functions expanded to contain input variables:

$$
\begin{array}{r}
2 \cdot v_{1}+5 \cdot v_{2}+2 \cdot v_{3}+2 \cdot v_{4}+2 \cdot v_{5} \\
-7.64
\end{array}
$$

For network output vector mapping function ${ }_{f_{2}} \aleph_{>1}^{\ell_{o u t}}$, experiment $\operatorname{Exp}_{21,848}^{\mathcal{E}_{C_{N}}, \mathrm{NN}: 5-1-2}$ yields the following solution that results in the image in Fig. 7.4 on page $119($ d):

$$
\begin{aligned}
& \mathcal{E}_{C_{N}}^{T r}=0.089: c_{1}=\frac{754}{884}, c_{2}=\frac{94}{97} \\
& \mathcal{E}_{C_{N}}^{T e}=0.112: c_{1}=\frac{180}{220}, c_{2}=\frac{23}{24}
\end{aligned}
$$

Output Neuron Functions expanded to contain input variables:

$$
\begin{array}{r}
300.36\left(v_{2}+v_{3}+v_{4}\right)+439.98 \\
-300.36\left(v_{2}+v_{3}+v_{4}\right)-446.62
\end{array}
$$

\subsubsection{Discussion of Insunza Results}

An overall summary of all of the broad scale error distributions is reported in Table 7.1 on page 110, in which the Insunza results are consistent with the overall rankings of neural network output vector mapping functions on training and testing 


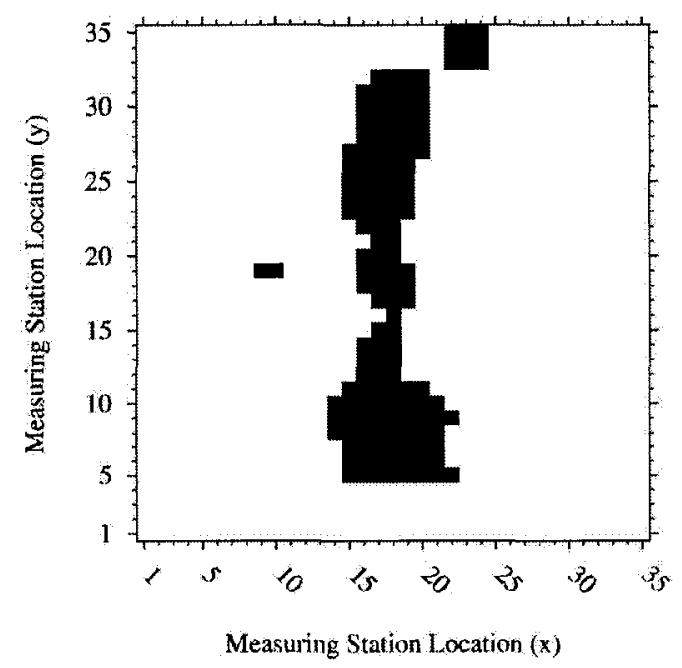

(a) Map for ${ }_{f_{1}} \aleph_{1}^{\ell_{\text {out }}}(x)\left(\mathcal{E}_{C}, \mathrm{NN}: 5-1-1\right)$

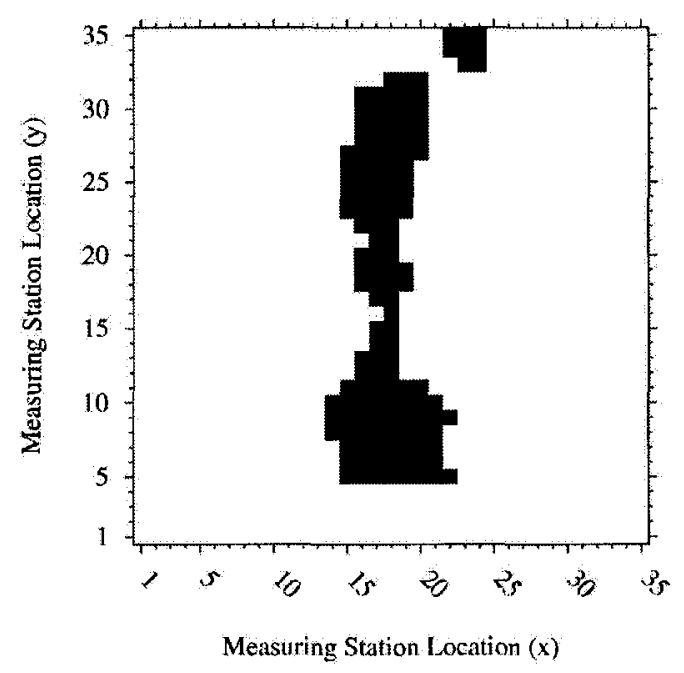

(c) Map for $f_{2} \aleph_{1}^{\ell_{\text {out }}}(x)\left(\mathcal{E}_{C}, \mathrm{NN}: 5-1-1\right)$

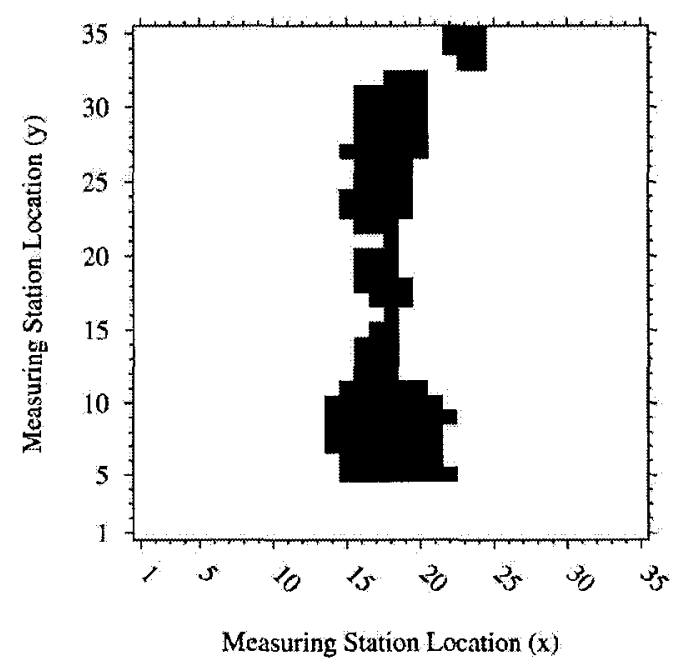

(b) Map for ${ }_{f_{1}} \mathcal{K}_{>1}^{\mathcal{E}_{\text {out }}}(x)\left(\mathcal{E}_{C}, \mathrm{NN}: 5-1-2\right)$

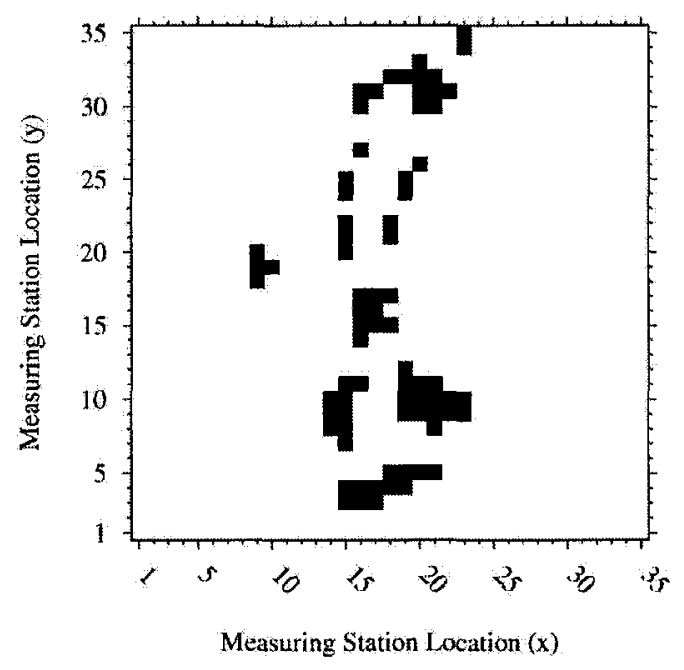

(d) Map for ${ }_{f_{2}} \mathrm{~K}_{>1}^{\text {out }}(x)\left(\mathcal{E}_{G}, \mathrm{NN}: 5-1-2\right)$

Figure 7.3: Selected Insunza Results $\left(\mathcal{E}_{C}\right)$. 


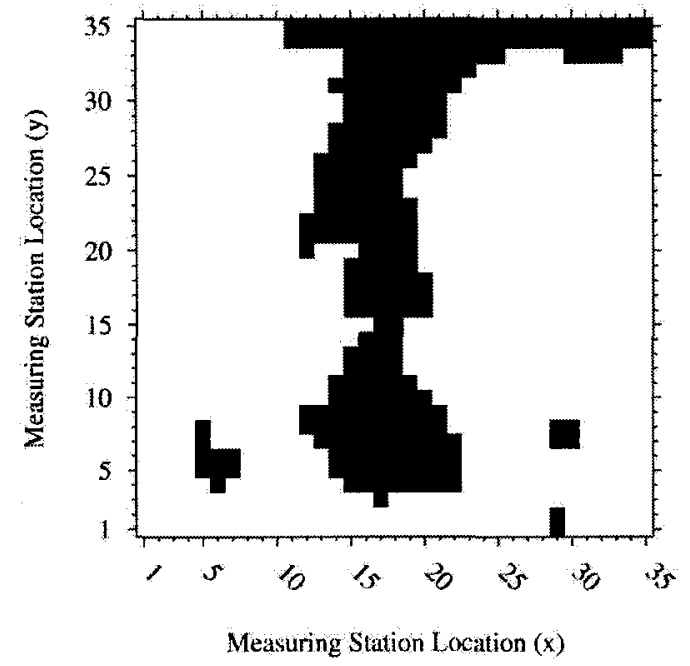

(a) Map for $f_{1} \aleph_{1}^{\mathcal{E}_{\text {out }}}(x)\left(\mathcal{E}_{C_{N}}, \mathrm{NN}: 5-1-1\right)$

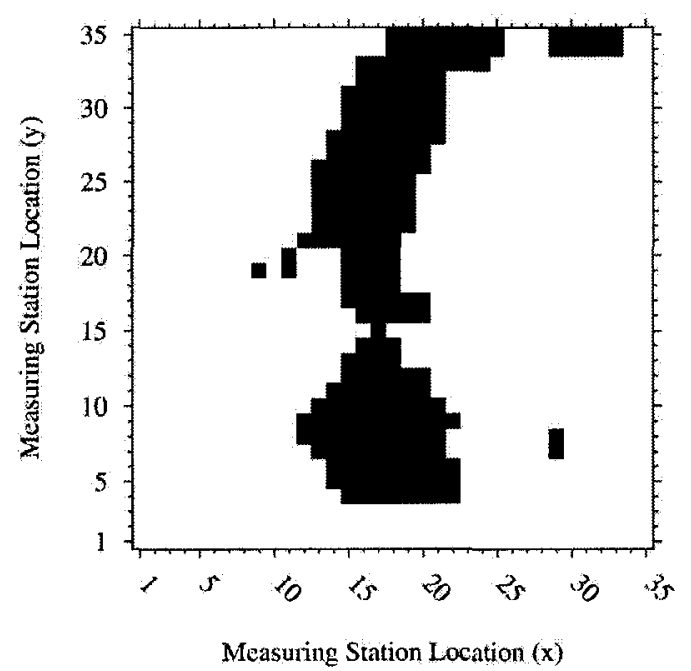

(c) Map for ${ }_{f_{2}} \aleph_{1}^{\mathcal{E}_{\text {out }}}(x)\left(\mathcal{E}_{C_{N}}, \mathrm{NN}: 5-1-1\right)$

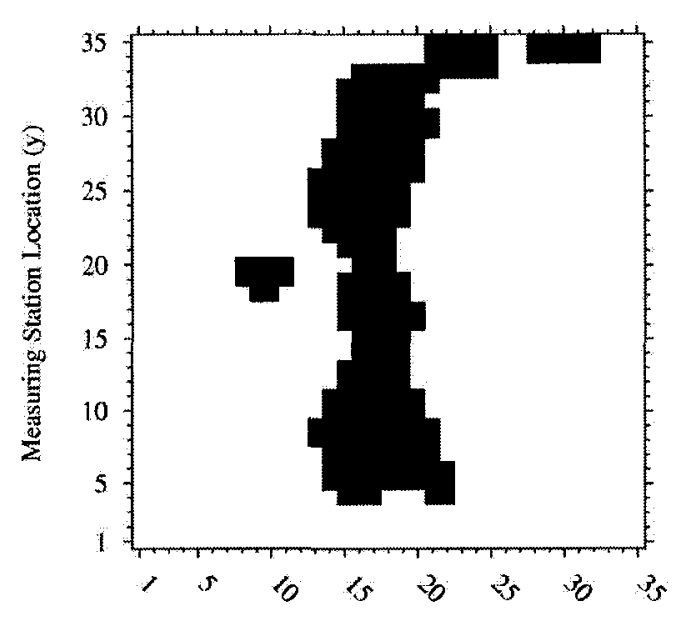

Measuring Station Location $(x)$

(b) Map for ${ }_{f_{1}} \aleph_{>1}^{\ell_{\text {out }}}(x)\left(\mathcal{E}_{C_{N}}, \mathrm{NN}: 5-1-2\right)$

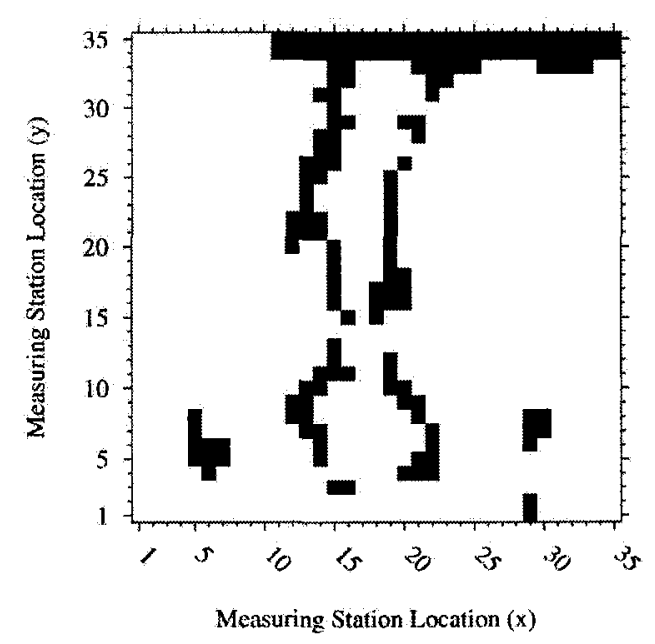

(d) Map for ${ }_{f_{2}} \aleph_{>1}^{\ell_{\text {out }}}(x)\left(\mathcal{E}_{C_{N}}, \mathrm{NN}: 5-1-2\right)$

Figure 7.4: Selected Insunza Results $\left(\mathcal{E}_{C_{N}}\right)$. 
data. In Fig. 7.1 on page 113 and Fig. 7.2 on page 114 each of the 8 specific error distributions each of size 11,520 are reported; one per row. Most of these error distributions are skewed towards the low error values; with some mapping functions having modes that are lower than others (e.g. $\approx 0.05$ vs. $\approx 0.45$ ). In order to be more quantitative, all of these distributions were compared with each other in a pairwise fashion. Table 7.2 on page 112 reports the probability that any two particular distributions being compared come from the same underlying distribution. All error distributions are significantly different except for comparing the training error distributions for $\mathcal{E}_{C_{N}}\left(f_{2} \aleph_{1}^{\ell_{\text {out }}}\right)$ vs. $\mathcal{E}_{C_{N}}\left(f_{1} \aleph_{>1}^{\ell_{\text {out }}}\right)$; that contains a probability of 0.42 that the error distributions come from the same underlying distribution. However, on testing data, the probability drops to 0.02 , indicating that 0.42 is likely to be an anomaly.

For each of the 8 reported error distributions, a single result has been selected (See Section 6.2.5 on page 107) and listed in Section 7.1.1.1 on page 115 with the associated variable semantics detailed in Table 6.1 on page 92 and Fig. 6.2 on page 92 . These 8 individual neural network solutions each have an associated analytic function ${ }^{1}$, which, in general, use fewer variables than the original number of variables present in the input data ${ }^{2}$. The variables and their frequencies within these 8 solutions are: $v_{1}(5), v_{2}(8), v_{3}(8), v_{4}(4)$, and $v_{5}(6)$. This indicates that $v_{2}$ and $v_{3}$ are the most frequent variables, and possibly the most influential for the Insunza problem. In addition, for each of the 8 specific solutions, their associated spatial maps were constructed and reported in Fig. 7.3 on page 118 and Fig. 7.4 on page 119. It can be seen that when comparing these to Fig. 6.1 on page 91, good reproducibility of the known cave location is performed along with a prediction of a location of an underground cave that was later confirmed by drilling a bore hole.

Future sections will discuss NN-GEP's parameter space Section 7.2.1 on page 134 and a comparison with other techniques Section 7.3 on page 141.

\footnotetext{
${ }^{1}$ The function may be considered as a new feature.

${ }^{2}$ Fewer variables indicates that NN-GEP is performing feature selection.
} 


\subsubsection{Breast Cancer Broad Scale Results}

The training and testing errors, along with a comparison between the two, for all neural network solutions are shown in Fig. 7.5 on page 123 and Fig. 7.6 on page 124 in a similar fashion as was done for the Insunza results. For the Breast cancer training and testing errors, it can be seen that they are all skewed towards lower error values except for Fig. $\mathbf{7 . 6}$ on page 124(a), which is more closely related to a uniform distribution. The best testing error distribution is in Fig. 7.5 on page 123(f) for the $\mathcal{E}_{C}^{T e}\left({ }_{f_{2}} \aleph_{1}^{\ell_{\text {out }}}\right)$ neural network output vector mapping function, with a very large percentage of the results having good testing errors.

A visual inspection of all of the figures in Fig. 7.5 on page 123 and Fig. 7.6 on page 124 may be performed in order to compare the distributions. However, a more statistical approach is to use the Komolgorov-Smirnov D Statistic in order to compare the distributions. This has been done for all pairwise possibilities (24 in total) and the results are reported in Table 7.3 on page 122. The two most similar distributions are for $\mathcal{E}_{C}\left({ }_{f_{2}} \aleph_{1}^{\ell_{\text {out }}}\right)$ and $\mathcal{E}_{C}\left({ }_{f_{1}} \aleph_{>1}^{\ell_{\text {out }}}\right)$ with training distribution comparison value 2.05 and testing distribution comparison value 3.46. This same observation applies to the normalized case $\left(\mathcal{E}_{C_{N}}\right)$ as was seen in the classical error case $\left(\mathcal{E}_{C}\right)$.

\subsubsection{Selected Breast Cancer Results}

Not all ( $\operatorname{card}($ Exps $)=92,160)$ experimental results may be reported, so a subset have been chosen for the Breast Cancer investigation (See Section 6.2.5 on page 107).

For network output vector mapping function ${ }_{f_{1}} \aleph_{1}^{\ell_{\text {out }}}$, experiment $\operatorname{Exp}_{8,047}^{\mathcal{E}_{C}, \mathrm{NN}: 9-2-1}$ yields the following solution (See Fig. $\mathbf{7 . 5}$ on page 123(a)):

$$
\begin{aligned}
& \mathcal{E}_{C}^{T r}=0.033: c_{1}=\frac{342}{356}, c_{2}=\frac{188}{192} \\
& \mathcal{E}_{C}^{T e}=0.007: c_{1}=\frac{87}{88}, c_{2}=\frac{47}{47}
\end{aligned}
$$

Output Neuron Functions expanded to contain input variables:

$$
-94.98 \cdot\left(v_{2}+v_{3}+v_{6}+9.57\right)
$$

For network output vector mapping function ${ }_{f_{2}} \aleph_{1}^{\ell_{\text {out }}}$, experiment $\operatorname{Exp}_{9,404}^{\mathcal{E}_{C}, \mathrm{NN}: 9-2-1}$ yields the following solution (See Fig. 7.5 on page 123(c)): 
Table 7.3: Pairwise comparison of Breast Cancer error distributions. KomolgorovSmirnov D Statistic and associated hypothesis testing probability (See Section 2.3.3 on page 37) for Pairwise Comparison of Training (upper triangular matrix) and Testing (lower triangular matrix) for two Error Distributions $\left(\mathcal{E}_{C}, \mathcal{E}_{C_{N}}\right)$. Large $\mathrm{D}$ values indicate the degree of difference in the distributions. Small probability values show that the CDF of the first error data set is significantly different than the second error data set. Each distribution contains 11,520 FFNN solution errors.

Komolgorov-Smirnov D Statistic

\begin{tabular}{|c|c|c|c|c|}
\hline & $\mathcal{E}_{C}\left({ }_{f_{\mathrm{l}}} \aleph_{1}^{\ell_{\text {out }}}\right)$ & $\mathcal{E}_{C}\left({ }_{f_{2}} \aleph_{1}^{\ell_{\text {out }}}\right)$ & $\mathcal{E}_{C}\left({ }_{f_{1}} \aleph_{>1}^{\ell_{\text {out }}}\right)$ & $\mathcal{E}_{C}\left({ }_{f_{2}} \aleph^{\ell_{\text {out }}}\right)$ \\
\hline $\mathcal{E}_{C}\left({ }_{f_{1}} \aleph_{1}^{\ell_{\text {out }}}\right)$ & & 48.32 & 46.80 & 25.79 \\
\hline $\mathcal{E}_{C}\left({ }_{f_{2}} \aleph_{1}^{\ell_{\text {out }}}\right)$ & 45.28 & & 2.05 & 24.20 \\
\hline $\mathcal{E}_{C}\left(W^{\ell_{\text {out }}}\right)$ & 43.92 & 3.46 & & 23.27 \\
\hline $\mathcal{E}_{C}\left({ }_{f_{2}} \aleph_{>1}^{\varepsilon_{\text {out }}}\right)$ & 21.78 & 25.20 & 22.69 & \\
\hline
\end{tabular}

\begin{tabular}{|c|c|c|c|c|}
\hline & $\mathcal{E}_{C_{N}}\left(f_{1} \aleph_{1}^{\ell_{\text {out }}}\right)$ & $\mathcal{E}_{C_{N}}\left({ }_{f_{2}} \aleph_{1}^{\ell_{\text {out }}}\right)$ & $\mathcal{E}_{C_{N}}\left(f_{1} \aleph_{>1}^{\ell_{\text {out }}}\right)$ & $\mathcal{E}_{C_{N}}\left({ }_{f_{2}} \aleph^{\ell_{\text {out }}}\right)$ \\
\hline$\overline{\mathcal{E}_{C_{N}}\left(f_{1} \aleph_{1}^{\ell_{\text {out }}}\right)}$ & & 49.07 & 47.77 & 26.20 \\
\hline $\mathcal{E}_{C_{N}}\left(f_{2} \aleph_{1}^{\mathcal{O}_{\text {out }}}\right)$ & 46.65 & & 2.36 & 24.40 \\
\hline $\mathcal{E}_{C_{N}}\left(f_{f_{1}} \aleph_{>1}^{\text {out }}\right)$ & 44.32 & 4.19 & & 23.17 \\
\hline $\mathcal{E}_{C_{N}}\left({ }_{f_{2}} \aleph_{>1}^{\text {out }}\right)$ & 23.95 & 25.21 & 22.58 & \\
\hline
\end{tabular}

Komolgorov-Smirnov Probability

\begin{tabular}{|c|c|c|c|c|}
\hline & $\mathcal{E}_{C}\left({ }_{f_{1}} \aleph_{1}^{\ell_{\text {out }}}\right)$ & $\mathcal{E}_{C}\left({ }_{f_{2}} \aleph_{1}^{\ell_{\text {out }}}\right)$ & $\mathcal{E}_{C}\left({ }_{f_{1}} \aleph_{>1}^{\ell_{\text {out }}}\right)$ & $\mathcal{E}_{C}\left({ }_{f_{2}} \aleph_{>1}^{\ell_{\text {out }}}\right)$ \\
\hline $\mathcal{E}_{C}\left({ }_{f} \aleph_{1}^{\varepsilon_{\text {out }}}\right)$ & & 0.00 & 0.00 & 0.00 \\
\hline $\mathcal{E}_{C}\left({ }_{f_{2}} \aleph_{1}^{\ell_{\text {out }}}\right)$ & 0.00 & & 0.00 & 0.00 \\
\hline $\mathcal{E}_{C}\left({ }_{f_{1}} \aleph_{>1}^{\ell_{\text {out }}}\right)$ & 0.00 & 0.00 & & 0.00 \\
\hline $\mathcal{E}_{C}\left({ }_{f_{2}} \aleph^{\mathcal{E}_{\text {out }}}\right)$ & 0.00 & 0.00 & 0.00 & \\
\hline
\end{tabular}

\begin{tabular}{|c|c|c|c|c|}
\hline & $\left.\mathcal{E}_{C_{N}\left(f_{1}\right.} \aleph_{1}^{\ell_{\text {out }}}\right)$ & $\mathcal{E}_{C_{N}}\left({ }_{f_{2}} \aleph_{1}^{\ell_{\text {out }}}\right)$ & $\mathcal{E}_{C_{N}}\left({ }_{f_{1}} \aleph_{>1}^{\ell_{\text {out }}}\right)$ & $\mathcal{E}_{C_{N}}\left({ }_{f_{2}} \aleph_{>1}^{\mathcal{E}_{\text {out } t}}\right)$ \\
\hline$\overline{\mathcal{E}}_{C_{N}}\left(f_{f} \aleph_{1}^{\ell_{\text {out }}}\right)$ & & 0.00 & 0.00 & 0.00 \\
\hline $\mathcal{E}_{C_{N}}\left(f_{f} \aleph_{1}^{\hat{\ell}_{\text {out }}}\right)$ & $\overline{0.00}$ & & 0.00 & 0.00 \\
\hline $\mathcal{E}_{C_{N}}\left(\boldsymbol{f}_{f_{1}} \aleph^{\ell_{\text {out }}}\right)$ & 0.00 & 0.00 & & 0.00 \\
\hline $\mathcal{E}_{C_{N}}\left({ }_{f_{2}} \aleph_{>1}^{\ell_{\text {out }}}\right)$ & 0.00 & 0.00 & 0.00 & \\
\hline
\end{tabular}




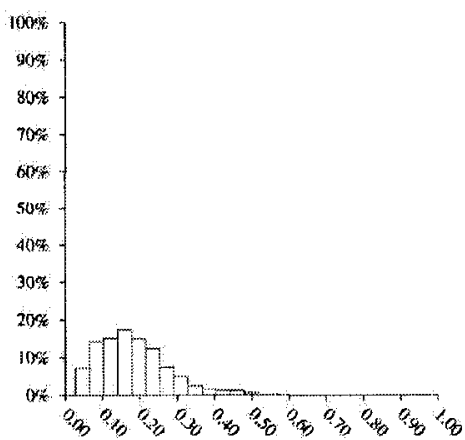

(a) $\mathcal{E}_{C}^{T r}\left({ }_{f_{1}} \aleph_{1}^{\ell_{\text {out }}}\right), \mathrm{NN}: 9-2-1$

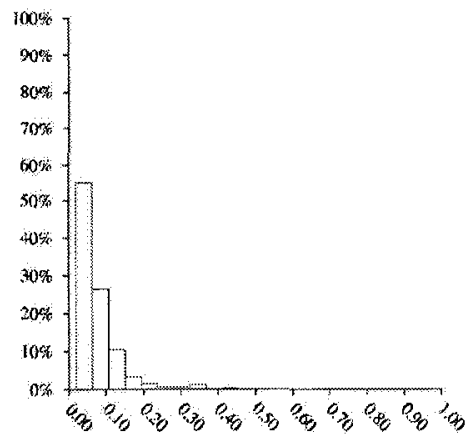

(d) $\mathcal{E}_{C}^{T r}\left({ }_{f_{2}} \aleph_{1}^{\ell_{\text {out }}}\right), \mathrm{NN}: 9-2-1$

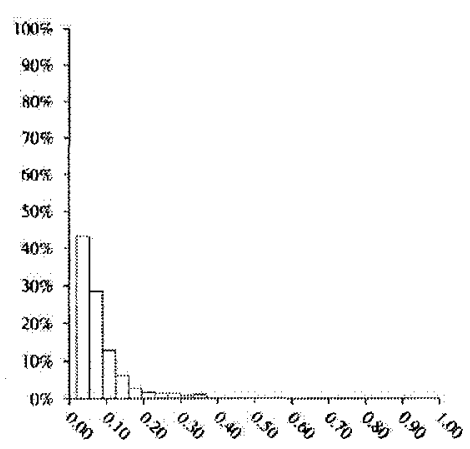

(g) $\mathcal{E}_{C}^{T r}\left({ }_{f_{1}} \aleph_{>1}^{\ell_{\text {out }}}\right), \mathrm{NN}: 9-2-2$

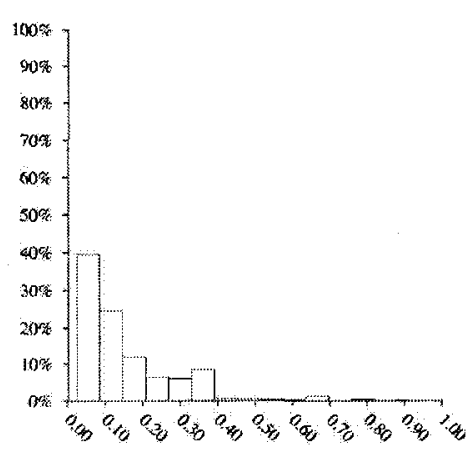

(j) $\mathcal{E}_{C}^{T r}\left({ }_{f_{2}} \aleph_{>1}^{\ell_{\text {out }}}\right), \mathrm{NN}: 9-2-2$

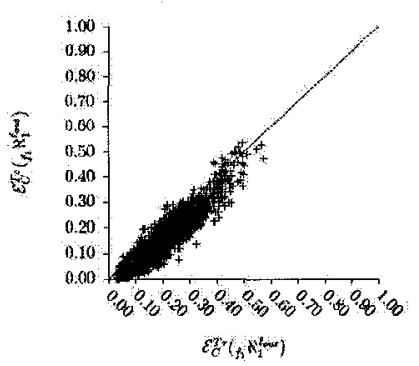

(b) Training vs, Testing

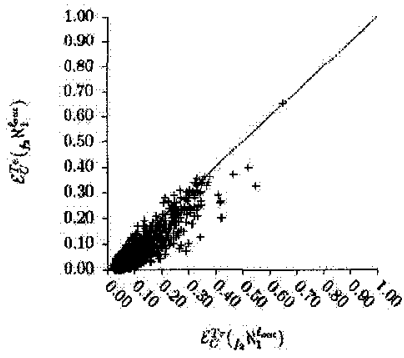

(e) Training vs. Testing

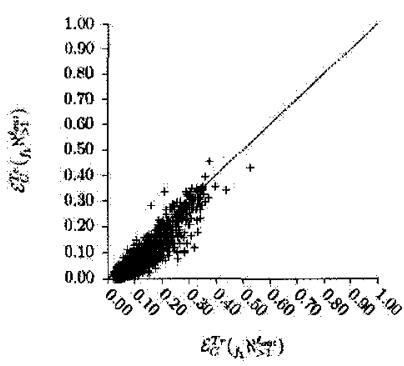

(b) Training vs, Testing

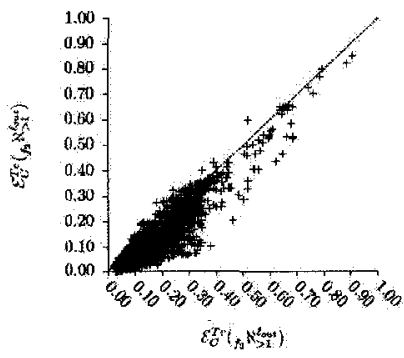

(k) Training vs. Testing

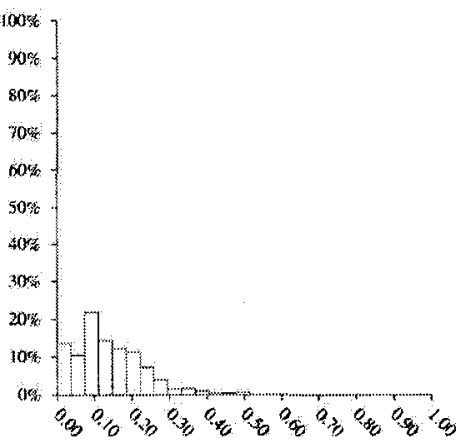

(c) $\mathcal{E}_{C}^{T e}\left({ }_{f_{1}} \aleph_{1}^{\ell_{\text {out }}}\right)$

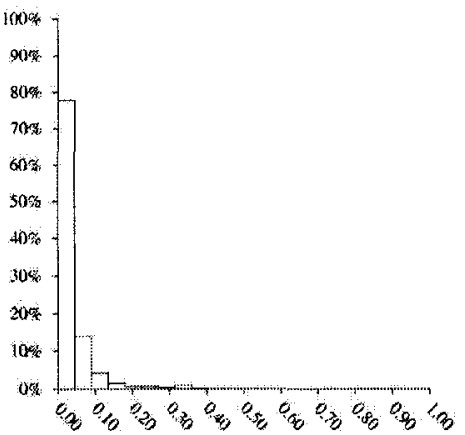

(f) $\mathcal{E}_{C}^{T e}\left({ }_{f_{2}} \aleph_{1}^{f_{\text {out }}}\right)$

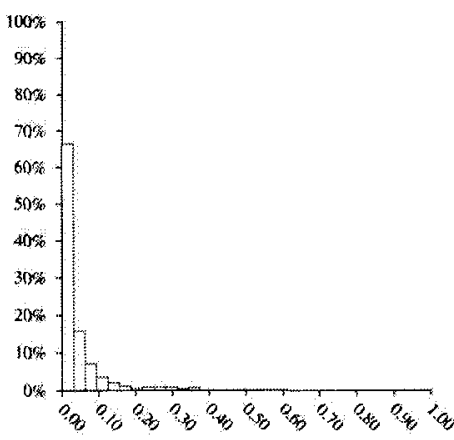

(i) $\mathcal{E}_{C}^{T e}\left({ }_{f_{1}} \aleph_{>1}^{\ell_{\text {out }}}\right)$

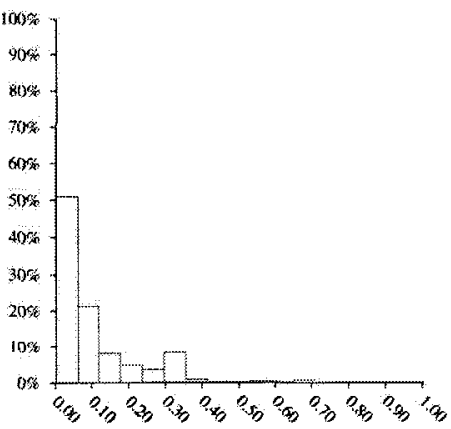

(l) $\mathcal{E}_{C}^{T}\left({ }_{f_{2}} \aleph^{\ell_{\text {out }}}\right)$

Figure 7.5: Breast Cancer $\left(\mathcal{E}_{C}\right)$. $(\operatorname{card}(\operatorname{Exps})=11,520)$. Sturges binning. 


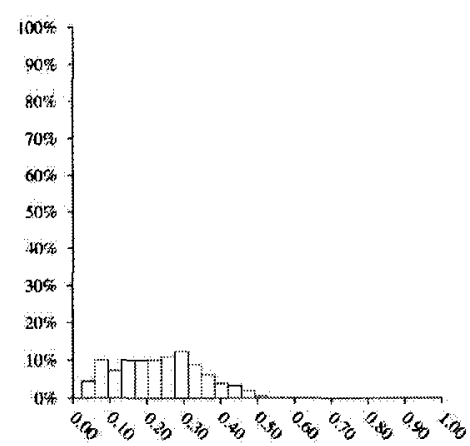

(a) $\mathcal{E}_{C_{N}}^{T_{r}}\left({ }_{f_{1}} \aleph_{1}^{\ell_{\text {out }}}\right), \mathrm{NN}: 9-2-1$

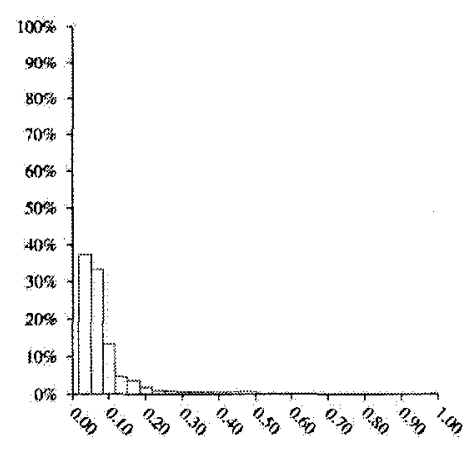

(d) $\mathcal{E}_{C_{N}}^{T r}\left(f_{2} \aleph_{1}^{\ell_{\text {out }}}\right), \mathrm{NN}: 9-2-1$

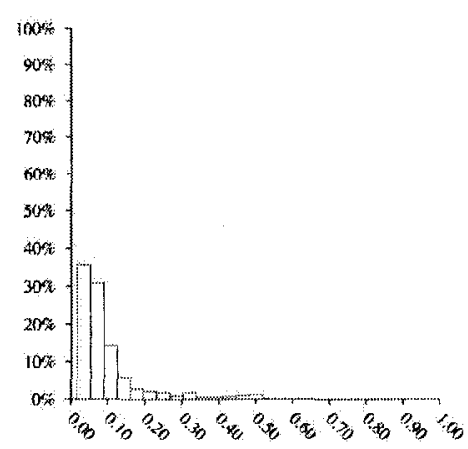

(g) $\mathcal{E}_{C_{N}}^{T r}\left(f_{1} \kappa_{>1}^{\ell_{\text {out }}}\right), \mathrm{NN}: 9-2-2$

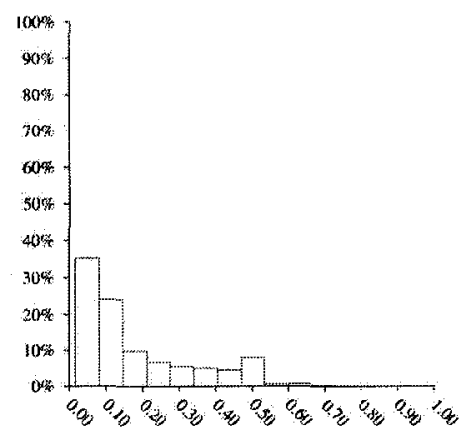

(j) $\mathcal{E}_{C_{N}}^{T r}\left(f_{2} \aleph_{>1}^{\ell_{\text {out }}}\right), \mathrm{NN}: 9-2-2$

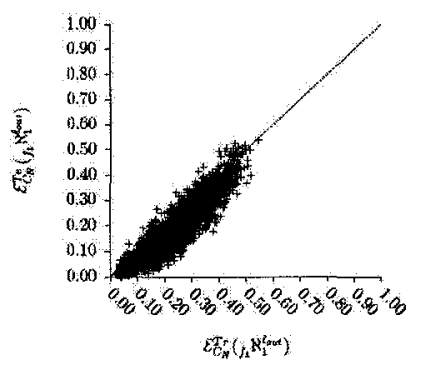

(b) Training vs. Testing

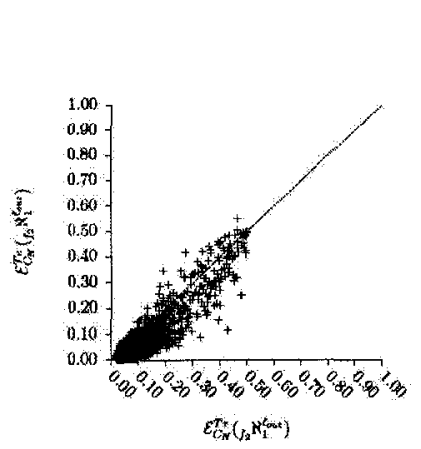

(e) Training vs. Testing

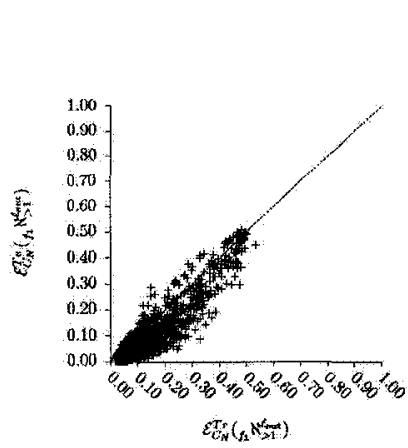

(h) Training vs. Testing

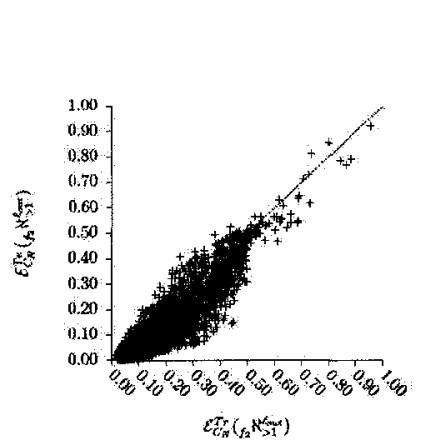

(k) Training vs. Testing

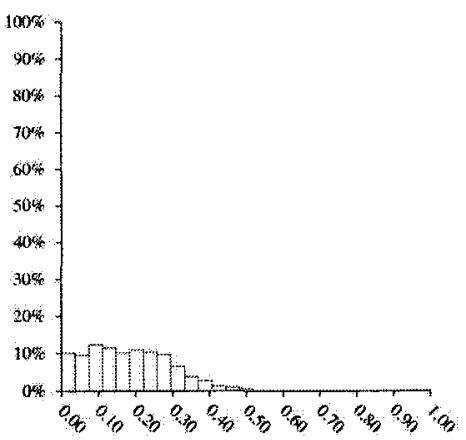

(c) $\mathcal{E}_{C_{N}}^{T e}\left(f_{1} \aleph_{1}^{\mathcal{C}_{\text {out }}}\right)$

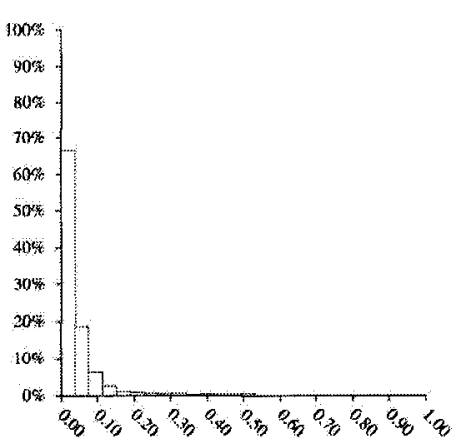

(f) $\mathcal{E}_{C_{N}}^{T e}\left(f_{2} K_{1}^{\ell_{\text {out }}}\right)$

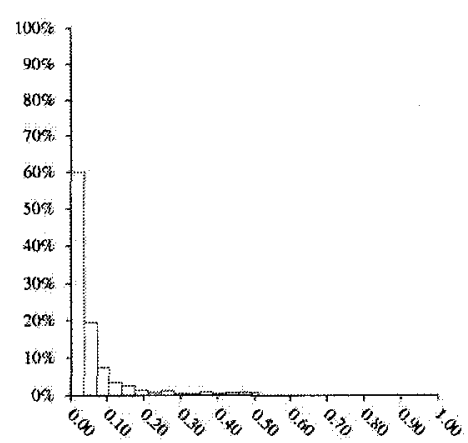

(i) $\mathcal{E}_{C_{N}}^{T e}\left(f_{1} \kappa_{>1}^{\ell_{\text {out }}}\right)$

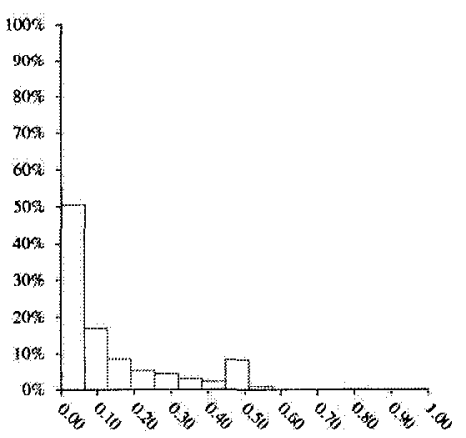

(l) $\mathcal{E}_{C_{N}}^{T e}\left(f_{2}{ }_{21}^{\ell_{\text {out }}}\right)$

Figure 7.6: Breast Cancer $\left(\mathcal{E}_{C_{N}}\right)$. (card(Exps) $\left.=11,520\right)$. Sturges binning. 


$$
\begin{aligned}
& \mathcal{E}_{C}^{T r}=0.027: c_{1}=\frac{345}{356}, c_{2}=\frac{188}{192} \\
& \mathcal{E}_{C}^{T e}=0: c_{1}=\frac{88}{88}, c_{2}=\frac{47}{47}
\end{aligned}
$$

Output Neuron Functions expanded to contain input variables:

$$
v_{1}+v_{2}+v_{3}+v_{6}+v_{7}+v_{8}-19.98
$$

For network output vector mapping function $f_{1} \aleph_{>1}^{\ell_{\text {out }} \text {, }}$, experiment $\operatorname{Exp}_{17,069}^{\mathcal{E}_{G}, \mathrm{NN}: 9-2-2}$ yields the following solution (See Fig. 7.5 on page 123(b)):

$$
\begin{aligned}
& \mathcal{E}_{C}^{T r}=0.031: c_{1}=\frac{344}{356}, c_{2}=\frac{187}{192} \\
& \mathcal{E}_{C}^{T e}=0: c_{1}=\frac{88}{88}, c_{2}=\frac{47}{47}
\end{aligned}
$$

Output Neuron Functions expanded to contain input variables:

$$
\begin{array}{r}
-5.13 \\
v_{1}+v_{3} \cdot\left(v_{6}+v_{2}\right)+v_{8}-27.08
\end{array}
$$

For network output vector mapping function ${ }_{f_{2}} \aleph_{>1}^{\ell_{\text {out }}}$, experiment $\operatorname{Exp}_{17,142}^{\mathcal{E}_{C}, \mathrm{NN}: 9-2-2}$ yields the following solution (See Fig. 7.5 on page 123(d)):

$$
\begin{aligned}
& \mathcal{E}_{C}^{T r}=0.0255: c_{1}=\frac{345}{356}, c_{2}=\frac{189}{192} \\
& \mathcal{E}_{C}^{T e}=0.0074: c_{1}=\frac{87}{88}, c_{2}=\frac{47}{47}
\end{aligned}
$$

Output Neuron Functions expanded to contain input variables:

$$
\begin{array}{r}
8.41\left(-v_{7} \cdot\left(v_{6}+v_{4}\right)-8.87 \cdot\left(v_{2}+v_{1}-9.88\right)\right) \\
-6.83\left(-v_{7} \cdot\left(v_{6}+v_{4}\right)-8.87 \cdot\left(v_{2}+v_{1}-9.88\right)\right)
\end{array}
$$

For network output vector mapping function ${ }_{f_{1}} \aleph_{1}^{\ell_{\text {out }}}$, experiment $\operatorname{Exp}_{17,009}^{\mathcal{E}_{\mathcal{C}_{N}, \mathrm{NN}: 9-2-1}}$ yields the following solution (See Fig. 7.6 on page 124(a)):

$$
\begin{aligned}
& \mathcal{E}_{C}^{T r}=0.0285: c_{1}=\frac{345}{356}, c_{2}=\frac{187}{192} \\
& \mathcal{E}_{C}^{T e}=0.0057: c_{1}=\frac{87}{88}, c_{2}=\frac{47}{47}
\end{aligned}
$$


Output Neuron Functions expanded to contain input variables:

$$
144.92 \cdot v_{6}+5.73
$$

For network output vector mapping function ${ }_{f_{2}} \aleph_{1}^{\ell_{\text {out }}}$, experiment $\operatorname{Exp}^{\mathcal{E}_{C_{N}}, \mathrm{NN}: 9-2-1}$ yields the following solution (See Fig. 7.6 on page 124(c)):

$$
\begin{aligned}
& \mathcal{E}_{C}^{T r}=0.0303: c_{1}=\frac{340}{356}, c_{2}=\frac{189}{192} \\
& \mathcal{E}_{C}^{T e}=0: c_{1}=\frac{88}{88}, c_{2}=\frac{47}{47}
\end{aligned}
$$

Output Neuron Functions expanded to contain input variables:

$$
2 \cdot v_{6}+v_{3}+v_{2}+v_{1}-14.33
$$

For network output vector mapping function ${ }_{f_{1}} \aleph_{>1}^{\ell_{\text {out }}}$, experiment $\operatorname{Exp}^{\mathcal{E}_{C_{N}, \mathrm{NN}: 9-2-2}}$ yields the following solution (See Fig. 7.6 on page 124(b)):

$$
\begin{aligned}
& \mathcal{E}_{C}^{T r}=0.0223: c_{1}=\frac{342}{356}, c_{2}=\frac{191}{192} \\
& \mathcal{E}_{C}^{T e}=0.0057: c_{1}=\frac{87}{88}, c_{2}=\frac{47}{47}
\end{aligned}
$$

Output Neuron Functions expanded to contain input variables:

$$
\begin{array}{r}
-v_{1}-v_{3}-v_{8}+9.16 \\
v_{2}+v_{5}+v_{6}-8.93
\end{array}
$$

For network output vector mapping function ${ }_{f_{2}} \aleph_{>1}^{\ell_{\text {out }}}$, experiment $\operatorname{Exp}^{\mathcal{E}_{C_{N}}, N N: 9-2-2}$ yields the following solution (See Fig. 7.6 on page $124(\mathrm{~d})$ ):

$$
\begin{aligned}
& \mathcal{E}_{C}^{T r}=0.0265: c_{1}=\frac{339}{356}, c_{2}=\frac{191}{192} \\
& \mathcal{E}_{C}^{T e}=0.0114: c_{1}=\frac{86}{88}, c_{2}=\frac{47}{47}
\end{aligned}
$$

Output Neuron Functions expanded to contain input variables:

$$
\begin{array}{r}
-45.56 \cdot\left(v_{1}+v_{2}+v_{6}\right)+441.69 \\
45.56 \cdot\left(v_{1}+v_{2}+v_{6}\right)-439.88
\end{array}
$$




\subsubsection{Discussion of Breast Cancer Results}

An overall summary of all of the broad scale error distributions is reported in Table 7.1 on page 110 , in which the Breast Cancer results are consistent with the overall rankings of neural network output vector mapping functions on training and testing data. In Fig. 7.5 on page 123 and Fig. 7.6 on page 124 each of the 8 specific error distributions each of size 11,520 are reported; one per row. Most of these error distributions are skewed towards the low error values; with some mapping functions having modes that are lower than others (e.g. $\approx 0.05$ vs. $\approx 0.20$ ). In order to be more quantitative, all of these distributions were compared with each other in a pairwise fashion. Table 7.3 on page 122 reports the probability that any two particular distributions being compared come from the same underlying distribution. All error distributions are significantly different (i.e. probability is 0.00 ) indicating that the neural network output mapping function is related to error distribution of neural network solutions.

For each of the 8 reported error distributions, a single result has been selected (See Section 6.2.5 on page 107) and listed in Section 7.1.2.1 on page 121 with the associated variable semantics detailed in Table 6.2 on page 94 and Fig. 6.4 on page 95. These 8 individual neural network solutions each have an associated analytic function ${ }^{3}$, which, in general, use fewer variables than the original number of variables present in the input data ${ }^{4}$. The variables and their frequencies within these 8 solutions are: $v_{1}(6), v_{2}(7), v_{3}(5), v_{4}(0), v_{5}(1), v_{6}(7), v_{7}(1), v_{8}(3)$, and $v_{9}(0)$. This indicates that $v_{1}, v_{2}, v_{3}$, and $v_{6}$ are the most frequent variables, and the most influential for the Breast Cancer problem. Also, $v_{4}$ and $v_{9}$ are not used at all, indicating their lack of influence upon good solutions.

Future sections will discuss NN-GEP's parameter space Section 7.2.2 on page 137 and a comparison with other techniques Section 7.3 on page 141; including a specific comparison between the simplest neural network solution found in Eq. 7.21 on page 126 containing one variable $\left(v_{6}\right)$ and [Brown, 2004]'s work in which a more complex solution involving an ensemble of neural networks is reported.

\footnotetext{
${ }^{3}$ The function may be considered as a new feature.

${ }^{4}$ Fewer variables indicates that NN-GEP is performing feature selection.
} 


\subsubsection{Werenskiold Broad Scale Results}

Collecting water samples in the Arctic on or near the Werenskiold glacier during the Spitzbergen scientific expedition in 1985 was not a trivial task. It may be possible that some of the data examples may be mislabeled. In any event, this section presents the results that have been obtained by NN-GEP within the broad scale experimentation methodology.

Fig. 7.7 on page 130 and Fig. 7.8 on page 131 show the distribution of training and testing errors and a comparison between the two. It can be seen that there are bimodal error distributions (e.g. Fig. 7.8 on page 131(j)) along with distributions that visually appear to be Gaussian (normal) (e.g. Fig. 7.8 on page 131(a)). It is also curious that there are examples of distributions that have a very large amplitude (e.g. Fig. 7.7 on page 130(a)).

A pairwise comparison of all training-to-training and testing-to-testing error distributions for both $\mathcal{E}_{C}$ and $\mathcal{E}_{C_{N}}$ was made using the Komolgorov-Smirnov D Statistic and reported in Table 7.4 on page 129. Compared to previous pairwise comparisons (e.g. Table 7.3 on page 122), these results indicate that the training and testing error distributions for $\mathcal{E}_{C}\left({ }_{f_{2}} \aleph_{1}^{\ell_{\text {out }}}\right)$ and $\mathcal{E}_{C}\left({ }_{f_{1}} \aleph_{>1}^{\ell_{\text {out }}}\right)$ are not as similar; 8.35 and 9.11 respectively.

The complete error distributions are shown in Fig. 7.7 on page 130 and Fig. 7.8 on page 131, but Table 7.7 on page 140 shows more clearly that ${ }_{f_{1}} \aleph_{>1}^{\ell_{\text {out }}}$ is the best performing strategy. Therefore, only selected results from that series of experiments $\left(f_{1} \aleph_{>1}^{\ell_{\text {out }}}\right)$ will be reported.

\subsubsection{Selected Werenskiold Results for ${ }_{f_{1}} \aleph_{>1}^{\ell_{\text {out }}}$}

Not all $(\operatorname{card}($ Exps $)=92,160)$ experimental results may be reported, so a subset have been chosen for the Werenskiold investigation (See Section 6.2.5 on page 107).

The minimum training error solution in Table 7.7 on page 140 is for $\operatorname{Exp}_{20,193}^{\mathcal{E}_{C}, \mathrm{NN}: 9-2-5}$ and network output vector mapping function ${ }_{f_{1}} \aleph_{>1}^{\ell_{\text {out }}}$ :

$$
\begin{aligned}
& \mathcal{E}_{C}^{T r}=0.18: c_{1}=\frac{26}{30}, c_{2}=\frac{24}{24}, c_{3}=\frac{0}{9}, c_{4}=\frac{8}{9}, c_{5}=\frac{19}{22} \\
& \mathcal{E}_{C}^{T e}=0.15: c_{1}=\frac{7}{7}, c_{2}=\frac{5}{5}, c_{3}=\frac{0}{1}, c_{4}=\frac{2}{2}, c_{5}=\frac{3}{5}
\end{aligned}
$$


Table 7.4: Pairwise comparison of Werenskiold error distributions. KomolgorovSmirnov D Statistic and associated hypothesis testing probability (See Section 2.3.3 on page 37) for Pairwise Comparison of Training (upper triangular matrix) and Testing (lower triangular matrix) for two Error Distributions $\left(\mathcal{E}_{C}, \mathcal{E}_{C_{N}}\right)$. Large values indicate the degree of difference in the distributions. Small probability values show that the CDF of the first error data set is significantly different than the second error data set. Each distribution contains 11,520 FFNN solution errors.

Komolgorov-Smirnov D Statistic

\begin{tabular}{|c|c|c|c|c|}
\hline & $\mathcal{E}_{C}\left({ }_{f_{1}} \aleph_{1}^{\ell_{\text {out }}}\right)$ & $\mathcal{E}_{C}\left({ }_{f_{2}} \aleph_{1}^{\ell_{\text {out }}}\right)$ & $\mathcal{E}_{C}\left(f_{1} \aleph_{>1}^{\ell_{\text {out }}}\right)$ & $\mathcal{E}_{C}\left(f_{2} \aleph_{>1}^{\ell_{\text {out }}}\right)$ \\
\hline $\mathcal{E}_{C}\left({ }_{f_{1}} \aleph_{1}^{\ell_{\text {out }}}\right)$ & & 42.18 & 45.43 & 18.35 \\
\hline $\mathcal{E}_{O}\left({ }_{f_{2}} \aleph_{1}^{\ell_{\text {out }}}\right)$ & 47.84 & & 8.35 & 30.44 \\
\hline $\mathcal{E}_{C}\left({ }_{f_{1}} \aleph_{>1}^{\ell_{\text {out }}}\right)$ & 48.57 & 9.11 & & 33.69 \\
\hline $\mathcal{E}_{C}\left({ }_{f_{2}} \aleph_{>1}^{\mathcal{E}_{\text {out }}}\right)$ & 22.59 & 30.78 & 29.45 & \\
\hline
\end{tabular}

\begin{tabular}{|c|c|c|c|c|}
\hline \multirow{2}{*}{$\overline{\mathcal{F}}\left(\mathcal{N}^{\ell o u t}\right)$} & $\mathcal{E}_{C_{N}}\left({ }_{f_{1}} \aleph_{1}^{\ell_{\text {out }}}\right.$ & $\mathcal{E}_{C_{N}}\left({ }_{f_{2}} \aleph_{1}^{\ell_{\text {out }}}\right)$ & $\mathcal{E}_{C_{N}}\left({ }_{f_{1}} \aleph_{>1}^{\ell_{\text {out }}}\right)$ & $\mathcal{E}_{C_{N}}\left({ }_{f_{2}} \aleph^{\ell_{\text {out }}}\right)$ \\
\hline & & 26.24 & 33.56 & 11.53 \\
\hline $\mathcal{E}_{C_{N}}\left({ }_{f_{2}} \aleph_{1}^{\aleph_{o u t}}\right)$ & 23.46 & & 23.43 & 29.54 \\
\hline $\mathcal{E}_{C_{N}}\left(f_{1} \aleph_{\geq 1}^{\mathcal{E}_{\text {out }}}\right)$ & 27.32 & 21.77 & & 32.99 \\
\hline${ }_{N}\left(f_{2} \aleph_{>1}^{\ell_{\text {out } t}}\right)$ & 6.58 & 27.89 & 29.32 & \\
\hline
\end{tabular}

Komolgorov-Smirnov Probability

\begin{tabular}{|c|c|c|c|c|}
\hline & $\mathcal{E}_{C}\left({ }_{f_{1}} \aleph_{1}^{\ell_{\text {out }}}\right)$ & $\mathcal{E}_{C}\left({ }_{f_{2}} \aleph_{1}^{\ell_{\text {out }}}\right)$ & $\mathcal{E}_{O}\left({ }_{f_{1}} \aleph_{>1}^{\ell_{\text {out }}}\right)$ & $\mathcal{E}_{C}\left({ }_{f_{2}} \aleph_{>1}^{\ell_{\text {out }}}\right)$ \\
\hline $\mathcal{E}_{C}\left({ }_{f_{1}} \aleph_{1}^{\ell_{\text {out }}}\right)$ & & 0.00 & 0.00 & 0.00 \\
\hline $\mathcal{E}_{C}\left({ }_{f_{2}} \aleph_{1}^{\mathcal{\ell}_{\text {out }}}\right)$ & 0.00 & & 0.00 & 0.00 \\
\hline $\mathcal{E}_{C}\left({ }_{f_{1}} \aleph^{\mathcal{E}_{>1}^{\text {out }}}\right)$ & 0.00 & 0.00 & & 0.00 \\
\hline $\mathcal{E}_{C}\left({ }_{f_{2}} \aleph^{\ell_{>1}^{\text {out }}}\right)$ & 0.00 & 0.00 & 0.00 & \\
\hline
\end{tabular}

\begin{tabular}{|c|c|c|c|c|}
\hline & $\| \mathcal{E}_{C_{N}}\left(f_{1} \aleph_{1}^{\ell_{\text {out }}}\right)$ & $\mathcal{E}_{C_{N}}\left({ }_{f_{2}} \aleph_{1}^{\text {out }}\right)$ & $\mathcal{E}_{C_{N}}\left(f_{1} \aleph_{>1}^{\ell_{\text {out }}}\right)$ & $\mathcal{E}_{C_{N}}\left({ }_{f_{2}} \aleph_{\ell_{>1} \text { out }}\right)$ \\
\hline$C_{N}(f$ & & 0.00 & 0.00 & 0.00 \\
\hline 裤 & 0.00 & & 0.00 & 0.00 \\
\hline$C_{N}\left(f_{1} N_{>1}^{e_{\text {out }}}\right)$ & 0.00 & 0.00 & & 0.00 \\
\hline${ }_{N}\left(f_{2} \aleph \aleph_{>1}^{\ell_{\text {out }}}\right)$ & 0.00 & 0.00 & 0.00 & \\
\hline
\end{tabular}




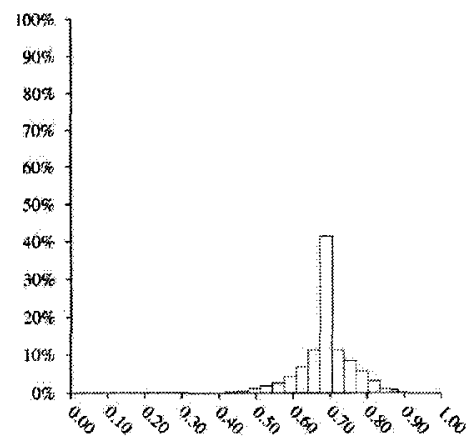

(a) $\mathcal{E}_{C}^{T r}\left({ }_{f_{1}} \aleph_{1}^{\ell \text { out }}\right), \mathrm{NN}: 9-2-1$

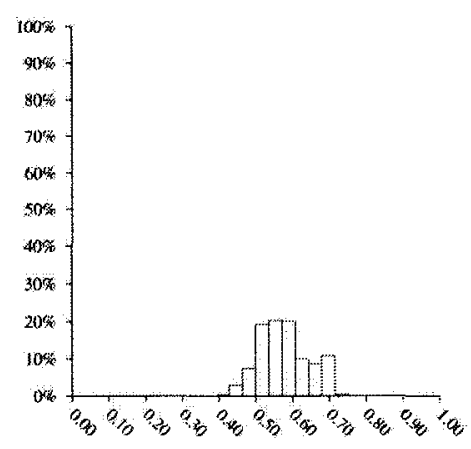

(d) $\mathcal{E}_{C}^{\operatorname{Tr}}\left({ }_{f_{2}} \aleph_{1}^{\ell_{\text {out }}}\right), \mathrm{NN}: 9-2-1$

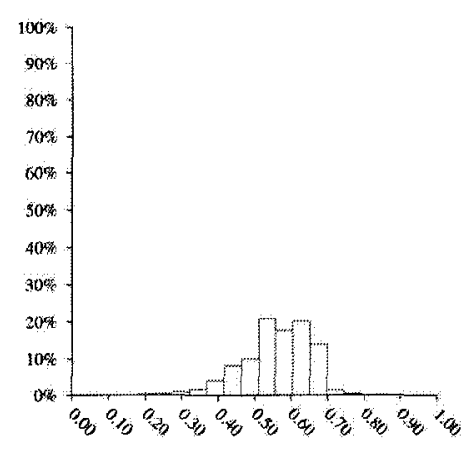

(g) $\mathcal{E}_{C}^{T r}\left({ }_{f_{1}} \aleph_{>1}^{\ell_{\text {out }}}\right), \mathrm{NN}: 9-2-5$

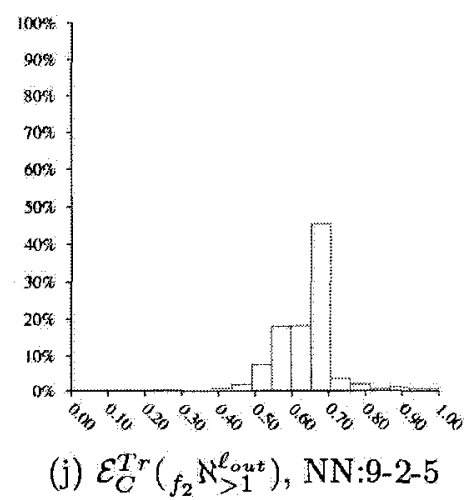

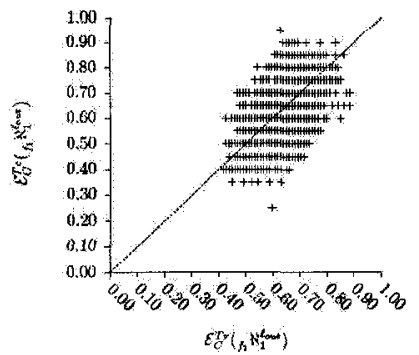

(b) Training vs. Testing

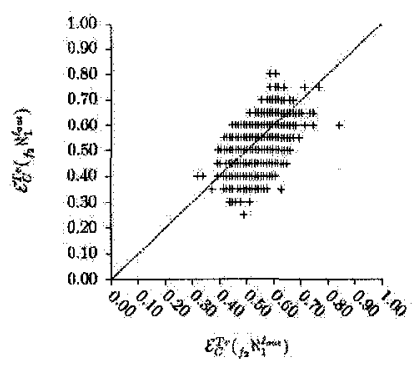

(e) Training vs. Testing

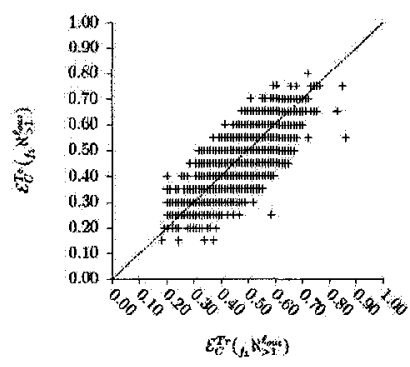

(h) Training vs. Testing

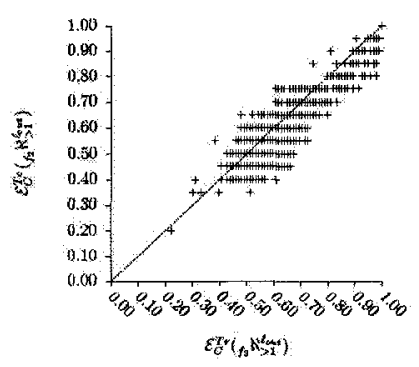

(k) Training vs. Testing

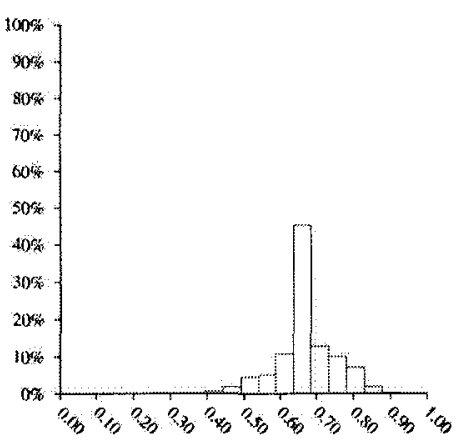

(c) $\mathcal{E}_{C}^{T e}\left({ }_{f_{1}} \aleph_{1}^{\ell_{\text {out }}}\right)$

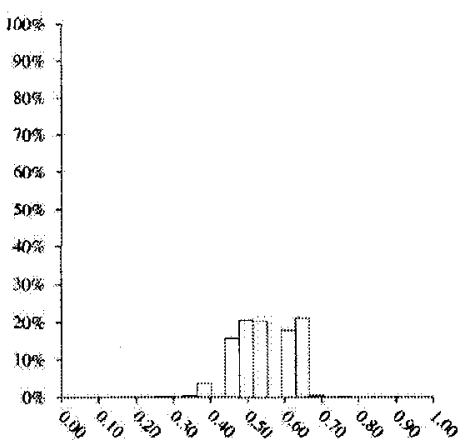

(f) $\mathcal{E}_{C}^{T e}\left({ }_{f_{2}} \aleph_{1}^{\ell_{\text {out }}}\right)$

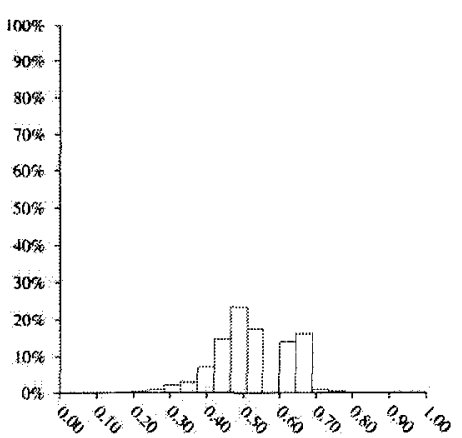

(i) $\mathcal{E}_{C}^{T e}\left({ }_{f_{1}} \aleph_{>1}^{\ell_{\text {out }}}\right)$

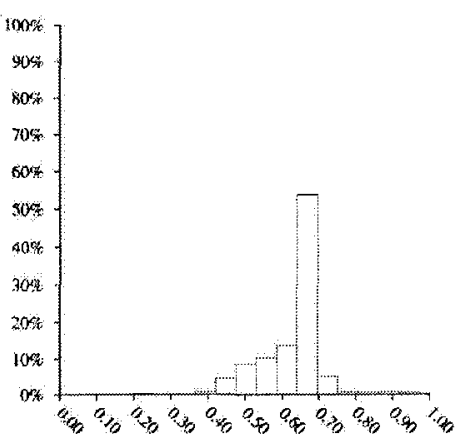

(l) $\mathcal{E}_{C}^{T e}\left({ }_{f_{2}} \aleph_{>1}^{\ell_{\text {out }}}\right)$

Figure 7.7: Werenskiold $\left(\mathcal{E}_{C}\right) .(\operatorname{card}(\mathrm{Exps})=11,520)$. Sturges binning. 


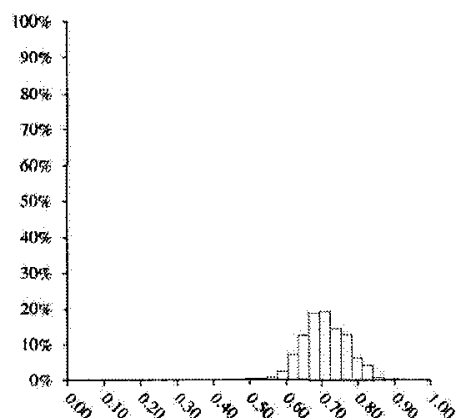

(a) $\mathcal{E}_{C_{N}}^{T r}\left({ }_{f_{1}} \aleph_{1}^{\ell \text { out }}\right), \mathrm{NN}: 9-2-1$
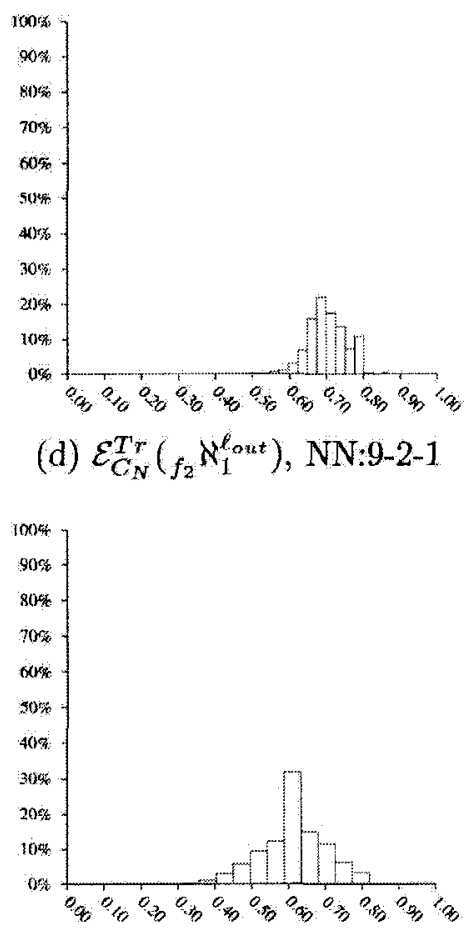

(g) $\mathcal{E}_{C_{N}}^{T r}\left(f_{1} \aleph_{>1}^{\ell_{\text {out }}}\right), \mathrm{NN}: 9-2-5$

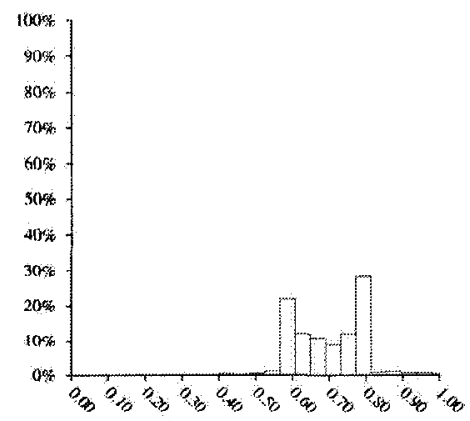

(j) $\mathcal{E}_{C_{N}}^{T r}\left({ }_{f_{2}} \aleph_{>1}^{\ell_{o n t}}\right), \mathrm{NN}: 9-2-5$

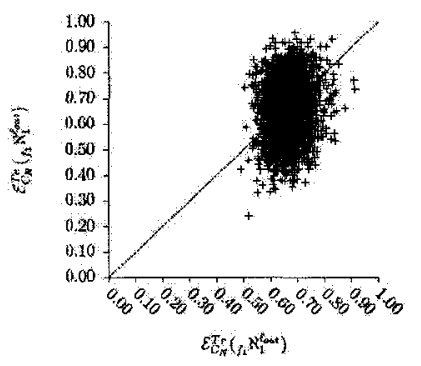

(b) Training vs. Testing

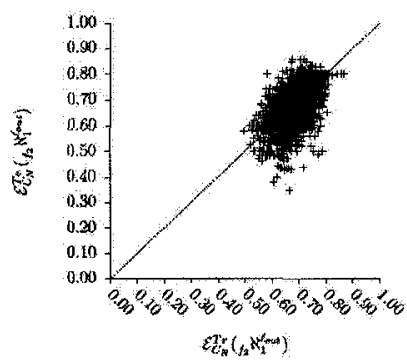

(e) Training vs. Testing

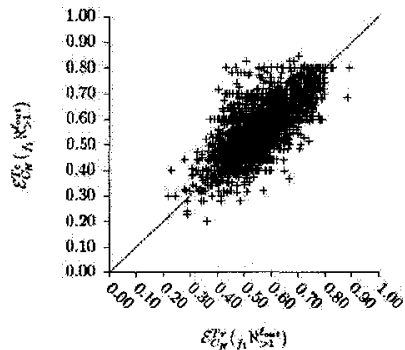

(h) Training vs. Testing

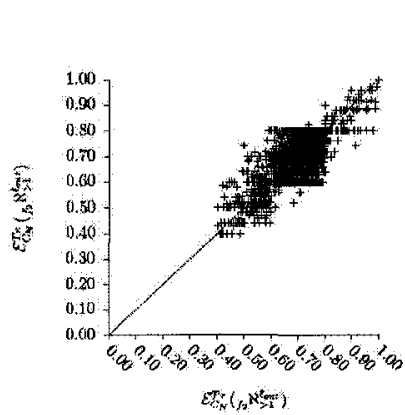

(k) Training vs. Testing

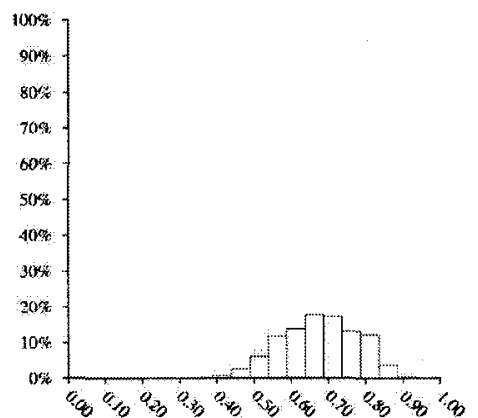

(c) $\mathcal{E}_{G_{N}}^{T}\left({ }_{f_{1}} \aleph_{1}^{\ell_{\text {out }}}\right)$

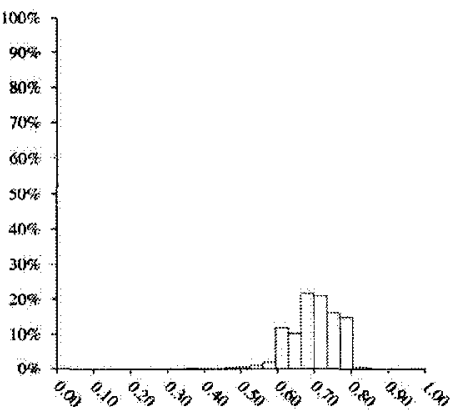

(f) $\mathcal{E}_{C_{N}}^{T e}\left(f_{2} \aleph_{1}^{\ell_{\text {out }}}\right)$

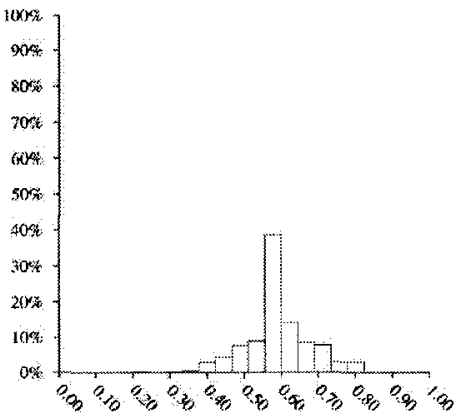

(i) $\mathcal{E}_{C_{N}}^{T \cdot e}\left(f_{1} \aleph_{>1}^{\ell_{\text {ost }}}\right)$

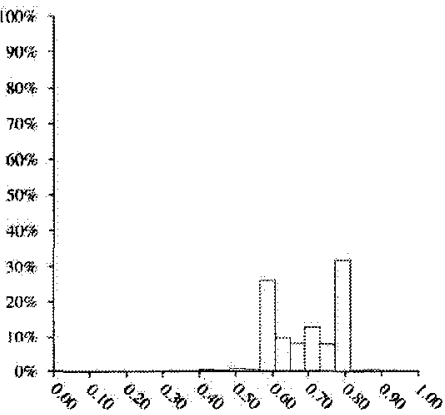

(l) $\mathcal{E}_{C_{N}}^{T e}\left(f_{2} \aleph_{>1}^{\ell_{\text {out }}}\right)$

Figure 7.8: Werenskiold $\left(\mathcal{E}_{C_{N}}\right)$. $(\operatorname{card}(\operatorname{Exps})=11,520)$. Sturges binning. 
Output Neuron Functions expanded to contain input variables:

$$
\begin{aligned}
& \left(v_{2}-7.02\right) \cdot\left(v_{4}+v_{1}-8.68\right) \cdot\left(v_{7}+v_{5}\right) \\
& \left(v_{2}-7.02\right) \cdot\left(v_{4}+v_{1}-8.68\right) \cdot\left(v_{7}+v_{5}\right) \cdot 7.12 \\
& \left(v_{2}-7.02\right) \cdot\left(v_{4}+v_{1}-8.68\right) \cdot\left(v_{7}+v_{5}\right)-10.04 \\
& 4 \cdot v_{4}+6 \cdot v_{1}-25.45 \\
& -3.86
\end{aligned}
$$

It is clear that Eq. $\mathbf{7 . 2 9}$ on page 132 will never be larger than Eq. 7.27 on page 132. This means that the network will never make any predictions for $c_{3}$, as can be seen empirically.

A close to minimum training error solution in Table 7.7 on page 140 is for $\operatorname{Exp}_{9,389}^{\mathcal{E}_{C}, \mathrm{NN}: 9-2-5}$ and network output vector mapping function ${ }_{f_{1}} \aleph_{>1}^{\ell_{\text {out }}}$ :

$$
\begin{aligned}
& \mathcal{E}_{C}^{T r}=0.19: c_{1}=\frac{28}{30}, c_{2}=\frac{24}{24}, c_{3}=\frac{9}{9}, c_{4}=\frac{0}{9}, c_{5}=\frac{15}{22} \\
& \mathcal{E}_{C}^{T e}=0.2: c_{1}=\frac{7}{7}, c_{2}=\frac{5}{5}, c_{3}=\frac{1}{1}, c_{4}=\frac{0}{2}, c_{5}=\frac{3}{5}
\end{aligned}
$$

Output Neuron Functions expanded to contain input variables:

$$
\begin{aligned}
& 7.88 \\
& 7.52 \cdot\left(2 \cdot v_{2}+v_{4}-v_{5}+v_{8}-22.11\right) \cdot\left(2 \cdot v_{2}+v_{4}-v_{5}+v_{8}-14.60\right) \\
& v_{7}^{2} \cdot\left(2 \cdot v_{2}+v_{4}-v_{5}+v_{8}-14.60\right) \\
& -9.41 \\
& 6 \cdot v_{2}+3 \cdot v_{4}-3 \cdot v_{5}+3 \cdot v_{8}-42.30
\end{aligned}
$$

It is clear that Eq. $\mathbf{7 . 3 5}$ on page 132 will never be larger than Eq. $\mathbf{7 . 3 2}$ on page 132. This means that the network will never make any predictions for $c_{4}$, as can be seen empirically.

\subsubsection{Discussion of Werenskiold Results}

An overall summary of all of the broad scale error distributions is reported in Table 7.1 on page 110 , in which the Werenskiold results are consistent with the overall 
rankings of neural network output vector mapping functions on training and testing data. In Fig. 7.7 on page 130 and Fig. 7.8 on page 131 each of the 8 specific error distributions each of size 11,520 are reported; one per row. These error distributions are not skewed towards the low error values and some are almost uniform; with modes that range from $\approx 0.55$ to $\approx 0.70$. This indicates that for this 5 class problem, absolute error values are quite high. However, the problem of collection of water samples is such that they are difficult to label properly. For example, for the collection of a water sample from a stream coming out from underneath a glacier, it is not clear for how long the water was in contact with the ground. This is important because the longer the contact, the larger the possibility that mineral content within the water will increase, hence altering the measured concentrations.

In order to be more quantitative, all of these distributions were compared with each other in a pairwise fashion. Table 7.4 on page 129 reports the probability that any two particular distributions being compared come from the same underlying distribution. All probabilities are 0.00 indicating that the underlying error distributions are all different and that the neural network output vector mapping function plays a key role.

For one of the 8 reported error distributions, namely, $f_{1} \aleph_{>1}^{\ell_{\text {out }}}$, two results were selected (See Section 6.2.5 on page 107) because of their low error values (See Table 7.7 on page 140) and listed in Section 7.1.3.1 on page 128 with the associated variable semantics detailed in Table 6.3 on page 97 and Fig. 6.7 on page 98 . These 2 individual neural network solutions each have an associated analytic function ${ }^{5}$, which, in general, use fewer variables than the original number of variables present in the input data ${ }^{6}$. The variables and their frequencies as reported in the 2 best Werenskiold neural networks are: $v_{1}(1), v_{2}(2), v_{3}(0), v_{4}(2), v_{5}(2), v_{6}(0), v_{7}(1), v_{8}(1)$, and $v_{9}$ (0). This indicates that $v_{2}, v_{4}$ and $v_{5}$ are the most influential in terms of usefulness for good neural network solutions. Table 6.3 on page 97 describes all of the variables. Future sections will discuss NN-GEP's parameter space Section $\mathbf{7 . 2 . 3}$ on page 139 and a comparison with other techniques Section 7.3 on page 141 .

\footnotetext{
${ }^{5}$ The function may be considered as a new feature.

${ }^{6}$ Fewer variables indicates that NN-GEP is performing feature selection.
} 


\subsection{Discussion of Parameter Space}

The previous section (Section 7.1 on page 109) gave an overview of all of the results within this thesis, including a sample of some of the best results. This section focuses on the parameter space. Are there any specific properties of the controlling parameters that consistently lead to good results? This is a difficult question, but an attempt is made for the 3 data sets by constructing artificial classes that represent 3 classes of results. The construction was performed via 2 linear discriminant thresholds (See Appendix C.7 on page 190). The construction of 3 dimensional spaces from high dimensions may be done with NN-GEP. However, it is not acceptable scientific practice to use a method to study itself. Instead, an independent method should be used for its study (See Section 2.3.4 on page 38). Within this thesis, the independent program has been reported in the literature (e.g. [Valdés, 2002], [Valdés, 2003], [Valdés and Barton, 2007]) and relies on an implementation of Hartigan's Leader Algorithm (See [Barton, 2004] for a description of it and other variants).

The 11 parameters that were investigated within the broad scale experimental methodology Table 6.6 on page 104 may be collected for a specific mapping function. Not all parameter spaces may be shown, but an inspection of Table 7.5 on page 135, Table 7.6 on page 137, and Table 7.7 on page 140 containing all of the details for each constructed class (as is explained in Appendix C.7 on page 190) was performed. It was found that the best network output mapping function is $f_{1} \aleph_{>1}^{\ell_{\text {out }}}$, and so the following subsections report results for it.

\subsubsection{Insunza Parameter Space}

Table 7.5 on page 135 shows the details of all of the 11,520 results for each of the 4 network output mapping functions when they are assigned to classes based on thresholds.

$f_{1} \aleph_{>1}^{\ell_{\text {out }}}$ was selected and the associated spaces were constructed Fig. 7.9 on page 136. The main observations are that good solutions are distributed throughout the parameter space and that with the elimination of the GEP Seeds controlling parameter, solutions are still distributed throughout the space. It is interesting that the granules in the top right figure within Fig. $\mathbf{7 . 9}$ on page 136 are oriented in such 
a way that a "good" portion of the investigated parameter space is at the top. This indicates that those subset of parameters may be the best choices when deciding to select controlling values for NN-GEP, but further work needs to be done in order to investigate this issue more thoroughly.

Table 7.5: Insunza Parameter Space Class Statistics for the 4 network output mapping functions and the 2 error measures $\left(\mathcal{E}_{C}, \mathcal{E}_{C_{N}}\right)$. Each group contains 11,520 neural network solutions.

If $\mathcal{E}_{C}^{T r}<0.05$ then good. If $\mathcal{E}_{C}^{T r}<0.15$ then ok. Otherwise bad.

\begin{tabular}{|c|c|c|c|c|c|c|c|c|}
\hline & Class & $\left\lfloor\mathcal{E}_{C}^{T r}\right\rfloor$ & $\overline{\mathcal{E}_{C}^{T r}}$ & $\left\lceil\mathcal{E}_{C}^{T r}\right\rceil$ & $\left\lfloor\mathcal{E}_{C}^{T e}\right\rfloor$ & $\overline{\mathcal{E}_{C}^{T e}}$ & $\left\lceil\mathcal{E}_{C}^{T e}\right\rceil$ & Count \\
\hline$k_{1}^{t_{0}}$ & good & 0.043 & 0.043 & 0.043 & 0.053 & 0.053 & 0.053 & $\overline{1}$ \\
\hline \multirow[t]{2}{*}{ NN:5-1-1 } & $o k$ & 0.051 & 0.096 & 0.107 & 0.024 & 0.095 & 0.098 & 10,486 \\
\hline & bad & 0.236 & 0.477 & 0.536 & 0.180 & 0.460 & 0.557 & 1,033 \\
\hline \multirow{3}{*}{$\begin{array}{l}f_{2} \aleph_{1}^{\ell_{\text {out }}} \\
\mathrm{NN}: 5-1-1\end{array}$} & good & 0.043 & 0.048 & 0.050 & 0.024 & 0.051 & 0.119 & 151 \\
\hline & $o k$ & 0.051 & 0.079 & 0.150 & 0.024 & 0.072 & 0.168 & 11,144 \\
\hline & bad & 0.151 & 0.330 & 0.755 & 0.061 & 0.257 & 0.639 & 225 \\
\hline \multirow{3}{*}{$\begin{array}{l}f_{1} \aleph_{>1}^{\ell_{\text {out }}} \\
\mathrm{NN}: 5-1-2\end{array}$} & good & 0.043 & 0.048 & 0.050 & 0.024 & 0.051 & 0.082 & 162 \\
\hline & $o k$ & 0.051 & 0.079 & 0.144 & 0.024 & 0.072 & 0.156 & 11,301 \\
\hline & bad & 0.160 & 0.331 & 0.587 & 0.090 & 0.277 & 0.516 & 57 \\
\hline \multirow{3}{*}{$\begin{array}{l}f_{2} \aleph_{i}^{K_{\text {out }}} \\
\text { NN:5-1-2 }\end{array}$} & good & 0.045 & 0.048 & 0.050 & 0.049 & 0.054 & 0.061 & 10 \\
\hline & $o k$ & 0.051 & 0.087 & 0.150 & 0.029 & 0.085 & 0.160 & 10,792 \\
\hline & bad & 0.151 & 0.516 & 0.985 & 0.074 & 0.439 & 0.963 & 718 \\
\hline
\end{tabular}

\begin{tabular}{|c|c|c|c|c|c|c|c|c|}
\hline & & & & & & & & \\
\hline & Class & $\left\lfloor\mathcal{E}_{C_{N}}^{T r}\right\rfloor$ & $\overline{\mathcal{E}_{C_{N}}^{T r}}$ & $\left\lceil\mathcal{E}_{C_{N}}^{\mathcal{T}^{T r}}\right\rceil$ & $\left\lfloor\mathcal{E}_{C_{N}}^{T e}\right\rfloor$ & $\overline{\mathcal{E}_{C_{T}}^{T e}}$ & $\left\lceil\mathcal{E}_{C_{N}^{T e}}^{T e}\right\rceil$ & Count \\
\hline$\psi^{\ell_{\text {out }}}$ & good & 0.071 & 0.071 & 0.071 & 0.117 & 0.117 & 0.117 & \\
\hline & & 074 & 1 & 0.15 & 7 & 0.169 & 299 & 245 \\
\hline & $a d$ & 0.150 & 32 & 0.8 & 82 & 423 & .574 & 11,274 \\
\hline & good & 0.058 & 0.067 & $\overline{0.0}$ & 0.084 & 0.128 & 0.215 & 109 \\
\hline & ok & 072 & .115 & 0.1 & 0.070 & 0.159 & 0.311 & 4,076 \\
\hline & bad & 50 & .254 & 0.65 & 0.053 & 0.250 & 0.639 & 7,335 \\
\hline$>1$ & good & 0.060 & 0.067 & 0.0 & 0.089 & 0.124 & 0.221 & 95 \\
\hline ง:5 & or & 072 & 0.116 & 0.150 & 0.073 & 0.159 & 0.420 & 3,877 \\
\hline & bad & 150 & 0.254 & 0.660 & 0.041 & 0.249 & 0.587 & 7,548 \\
\hline$f_{2} \aleph_{>1}^{\ell_{\text {out }}}$ & good & 35 & 0.068 & 0. & 0.100 & 0.128 & 0.171 & 4 \\
\hline & $o$ & & .1 & & 0.059 & 0.176 & 0.283 & 1,479 \\
\hline & bad & 0,150 & 0.321 & 0.941 & 0.080 & 0.326 & 0.907 & 0,037 \\
\hline
\end{tabular}




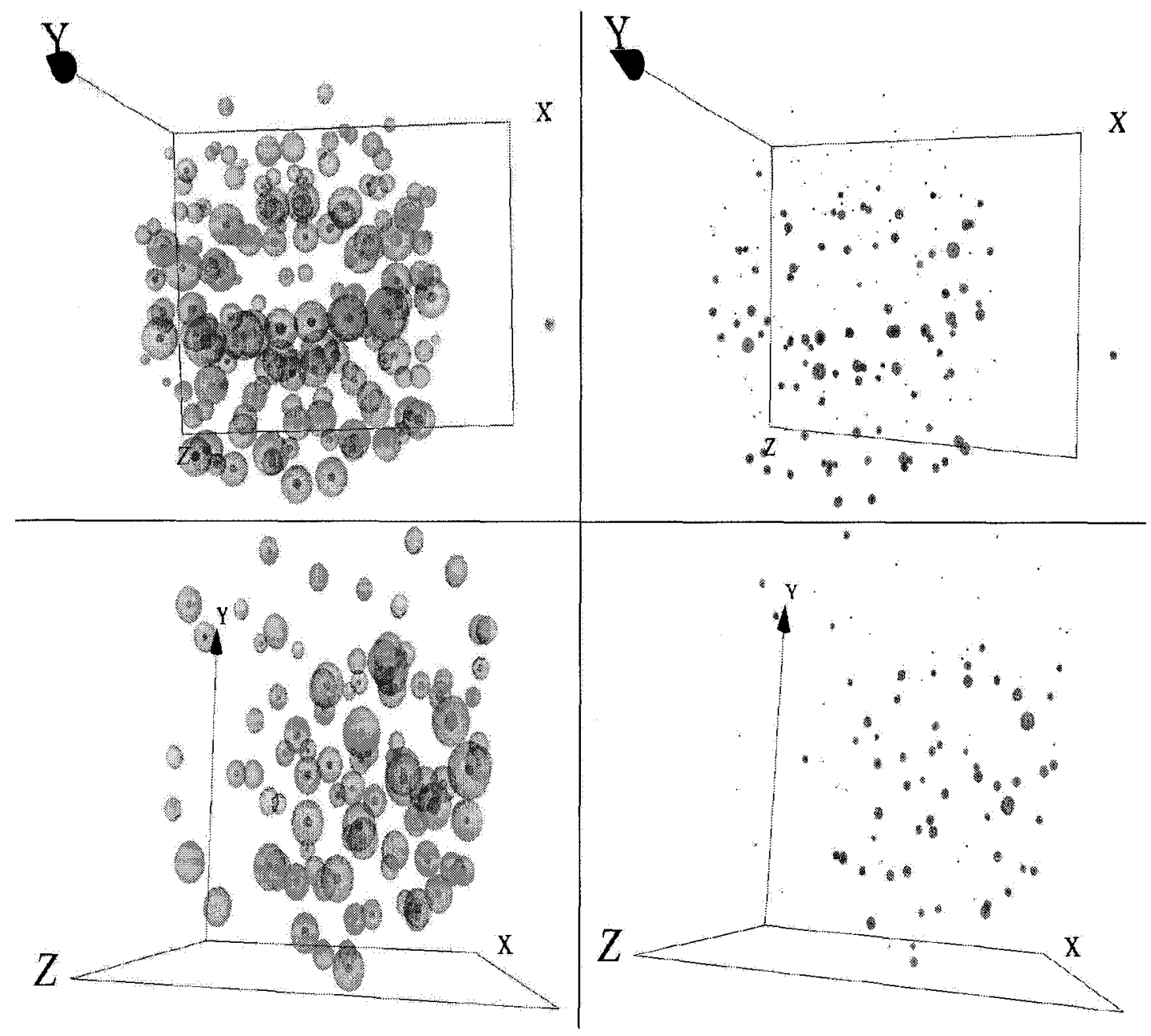

Figure 7.9: Insunza 3D Spaces for NN-GEP controlling parameters. There are 3 coloured spheres; one for each class (See Appendix C.7 on page 190). Sphere sizes are determined by the number of objects within a particular granule that have that associated class. In this figure, the largest spheres are class 2 (ok neural network solutions) and have been unwrapped in the right column in order to more clearly show the relation between good solutions (class 1 are small dark spheres) and bad solutions (class 3 ).

Top Row: 11D to 3D maps for all 11 controlling parameters (See Table 6.6 on page 104).

Bottom Row: 10D to 3D maps for 10 controlling parameters; the GEP Seeds parameter was removed.

Left Column: All classes within granules formed by the Leader Algorithm.

Right Column: Second and third most important classes within granules formed by the Leader Algorithm. Very tiny black spheres indicate the locations of the leader granules that only contain objects belonging to the same class (i.e. the granules are homogeneous). 


\subsubsection{Breast Cancer Parameter Space}

Table 7.6 on page 137 contains the statistics for all of the classes that have been artificially constructed over the set of solutions for each of the mapping functions and error measures. ${ }_{f_{1}} \aleph_{>1}^{\ell_{\text {out }}}$ is one of the best performing mapping functions and was selected. Fig. 7.10 on page 138 shows the 11D parameter space mapped into 3D. It also shows, on the bottom row, a reduced parameter space of $10 \mathrm{D}$ that is mapped into 3D in order to show the effect, or lack thereof, for the GEP Seeds controlling parameter.

Table 7.6: Breast Cancer Parameter Space Class Statistics.

If $\mathcal{E}_{C}^{T r}<0.028$ then good. If $\mathcal{E}_{C}^{T r}<0.15$ then ok. Otherwise bad.

\begin{tabular}{|c|c|c|c|c|c|c|c|c|}
\hline & Class & $\left\lfloor\mathcal{E}_{C}^{T r}\right\rfloor$ & $\overline{\mathcal{E}_{C}^{T r}}$ & $\left\lceil\mathcal{E}_{C}^{T r}\right\rceil$ & $\left\lfloor\mathcal{E}_{C}^{T e}\right\rfloor$ & $\overline{\mathcal{E}_{C}^{T e}}$ & $\left\lceil\mathcal{E}_{C}^{T e}\right\rceil$ & Count \\
\hline \multirow{3}{*}{$\begin{array}{l}f_{1} N_{1}^{N_{\text {fout }}} \\
\mathrm{NN}: 9-2-1\end{array}$} & good & 0.027 & 0.027 & 0.027 & 0.015 & 0.015 & 0.015 & 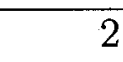 \\
\hline & $o k$ & 0.029 & 0.100 & 0.150 & 0 & 0.064 & 0.192 & 4,979 \\
\hline & bad & 0.151 & 0.243 & 0.573 & 0.044 & 0.191 & 0.533 & 6,539 \\
\hline \multirow{3}{*}{$\begin{array}{l}f_{2} \aleph_{1}^{\aleph_{\text {out }}} \\
\text { NN:9-2-1 }\end{array}$} & good & 0.020 & 0.026 & 0.027 & 0 & 0.014 & 0.022 & 463 \\
\hline & $o k$ & 0.029 & 0.065 & 0.150 & 0 & 0.029 & 0.192 & 10,129 \\
\hline & bad & 0.151 & 0.241 & 0.650 & 0.030 & 0.174 & 0.652 & 928 \\
\hline \multirow{3}{*}{ 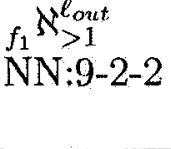 } & good & 0.020 & 0.026 & 0.027 & 0 & 0.013 & 0.030 & 417 \\
\hline & $o k$ & 0.029 & 0.065 & 0.150 & 0 & 0.028 & 0.163 & 9,820 \\
\hline & bad & 0.151 & 0.231 & 0.527 & 0.022 & 0.176 & 0.452 & 1,283 \\
\hline \multirow{3}{*}{$\begin{array}{l}f_{2} N_{>1} \text { out } \\
\text { NN:9-2-2 }\end{array}$} & good & 0.022 & 0.026 & 0.027 & $\overline{0}$ & 0.015 & 0.022 & 115 \\
\hline & $o k$ & 0.029 & 0.079 & 0.150 & 0 & 0.040 & 0.2 & 7,473 \\
\hline & bad & 0.151 & 0.291 & 0.909 & 0.022 & 0.236 & 0.852 & 3,932 \\
\hline
\end{tabular}

\begin{tabular}{|c|c|c|c|c|c|c|c|c|}
\hline \multicolumn{9}{|c|}{ len ok. Otherwise bad. } \\
\hline & Class & $\left\lfloor\mathcal{E}_{C_{N}}^{T r}\right\rfloor$ & $\overline{\mathcal{E}_{C_{N}}^{T r}}$ & $\left\lceil\mathcal{E}_{C_{N}}^{T r}\right\rceil$ & $\left\lfloor\mathcal{E}_{C_{N}}^{T e}\right\rfloor$ & $\overline{\mathcal{E}_{C_{N}}^{T e}}$ & $\left\lceil\mathcal{E}_{C_{N}}^{T e}\right\rceil$ & Count \\
\hline \multirow{3}{*}{$\begin{array}{l}f_{1} \aleph_{1}^{f_{\text {out }}} \\
\mathrm{NN}: 9-2-1\end{array}$} & good & & & & & & & \\
\hline & $o k$ & 24 & 092 & 0.150 & 0 & 0.055 & 0.202 &, 953 \\
\hline & bad & & 281 & 540 & 0.027 & 0.225 & .536 & 567 \\
\hline \multirow{3}{*}{$\begin{array}{l}f_{2} \aleph_{1}^{\ell_{\text {out }}} \\
\text { NN:9-2-1 }\end{array}$} & good & 0.017 & 0.017 & 7 & 0.016 & 0.016 & $\overline{0.016}$ & 1 \\
\hline & $o k$ & & 2 & & 0 & 0.029 & 202 & 0,154 \\
\hline & bad & & 273 & 0.503 & 27 & 0.204 & 550 & 365 \\
\hline \multirow{3}{*}{$\begin{array}{l}f_{1} \aleph_{>1}^{\ell_{o u t}} \\
\text { NN:9-2-2 }\end{array}$} & good & 7 & 0.017 & & 0 & 0 & 0 & 2 \\
\hline & $o k$ & & 0.066 & & 0 & 0.031 & 288 & 820 \\
\hline & bad & 0 & 0.289 & .536 & 027 & 0.221 & 0.511 & 1,698 \\
\hline \multirow{3}{*}{ 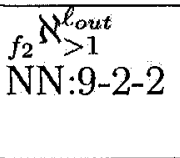 } & good & & .01 & & 0.022 & 0.022 & 0.022 & 1 \\
\hline & oh & & 0.0 & & 0 & 0.0 & 0.208 & 895 \\
\hline & bad & .15 & 0.340 & .956 & 0.027 & 0.275 & 0.926 & 4,624 \\
\hline
\end{tabular}




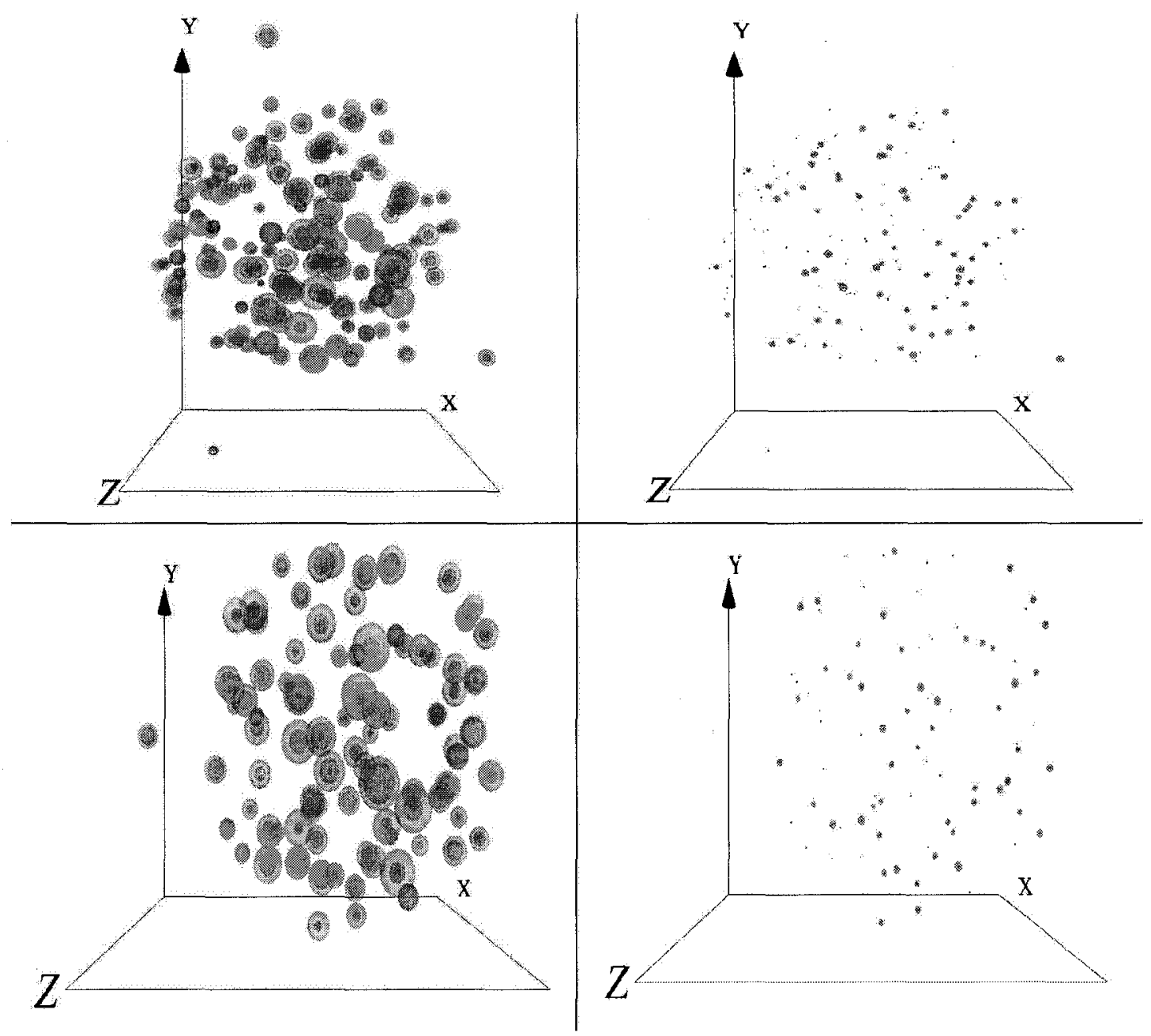

Figure 7.10: Breast Cancer 3D Spaces for NN-GEP controlling parameters. The right column has spheres that only represent class 1 granules. Only the third most important objects within the granules are shown. Good solutions are distributed throughout the parameter space. The elimination of the GEP Seeds parameter (bottom row) does not seem to significantly affect the distribution of good results on a global scale. Compare with Fig. $\mathbf{7 . 9}$ on page 136 that shows the third most important objects in the right column. 


\subsubsection{Werenskiold Parameter Space}

Collecting water samples in the Arctic on or near the Werenskiold glacier during the Spitzbergen scientific expedition in 1985 was not a trivial task. It may be possible that some of the data examples may be mislabeled. Table 7.7 on page 140 contains the statistics for the constructed classes (See Appendix C.7 on page 190). $f_{1} \aleph_{>1}^{N_{\text {out }}^{\text {out }} \text { is quite }}$ clearly the best performing mapping function, with $\left\lfloor\mathcal{E}_{C}^{T r}\right\rfloor=0.181$ and $\left\lfloor\mathcal{E}_{C}^{T e}\right\rfloor=0.353$. Fig. 7.11 on page 139 shows a representation of the 11,520 neural network solutions in terms of their 11 controlling parameters. It can be seen that good results are spread throughout the parameter space.

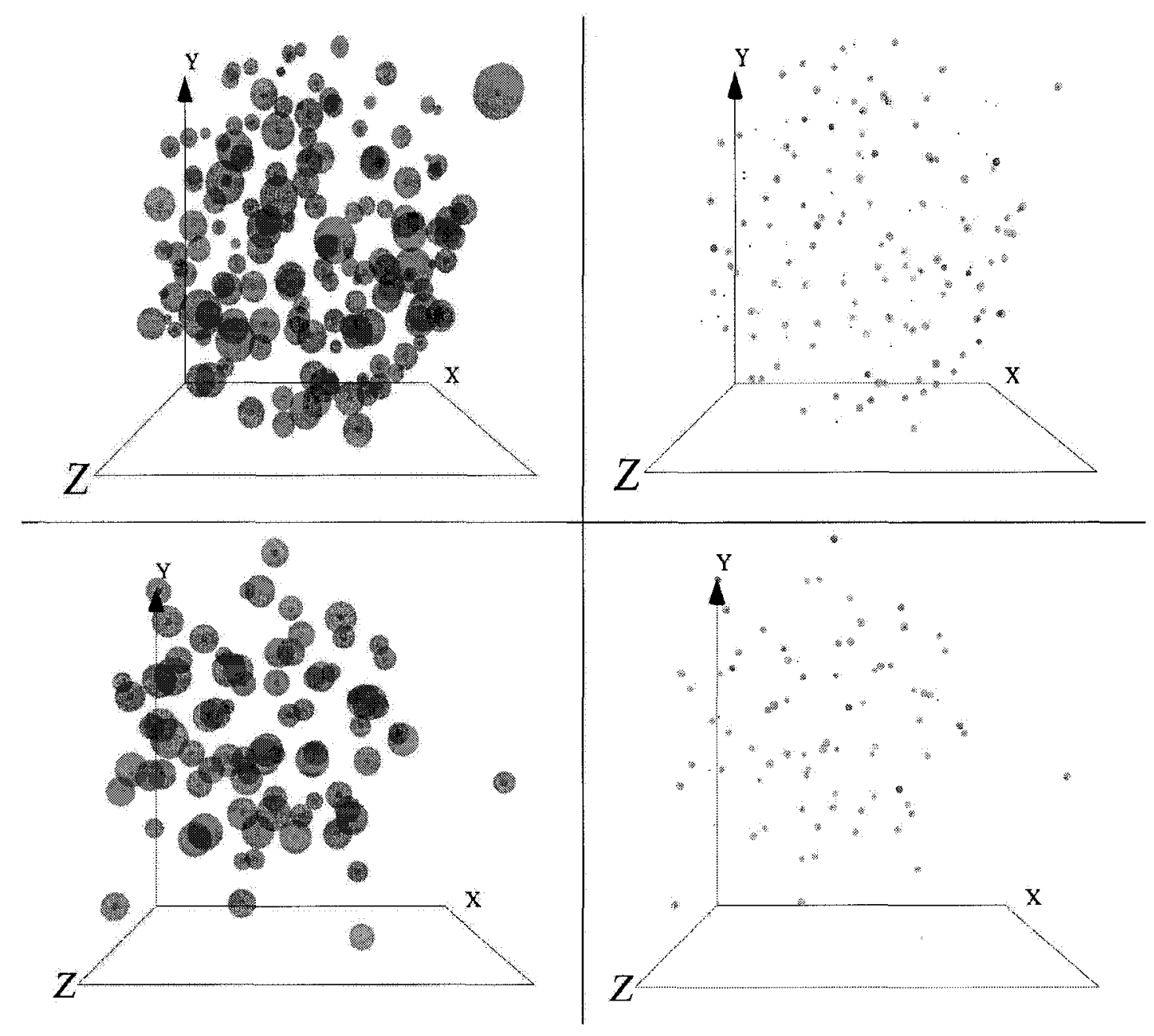

Figure 7.11: Werenskiold 3D Spaces for NN-GEP controlling parameters. Good results are spread throughout the parameter space. 
Table 7.7: Werenskiold Parameter Space Class Statistics.

If $\mathcal{E}_{C}^{T r}<0.42$ then good. If $\mathcal{E}_{C}^{T r}<0.50$ then ok. Otherwise bad.

\begin{tabular}{lc|rcr|rrr|r} 
& Class & $\left\lfloor\mathcal{E}_{C}^{T r}\right\rfloor$ & $\overline{\mathcal{E}_{C}^{T r}}$ & $\left\lceil\mathcal{E}_{C}^{T r}\right\rceil$ & $\left\lfloor\mathcal{E}_{C}^{T e}\right\rfloor$ & $\overline{\mathcal{E}_{C}^{T e}}$ & $\left\lceil\mathcal{E}_{C}^{T e}\right\rceil$ & Count \\
\hline$f_{1} \aleph_{1}^{\ell_{\text {out }}}$ & good & 0.415 & 0.415 & 0.415 & 0.4 & 0.4 & 0.4 & 2 \\
$\mathrm{NN}: 9-2-1$ & ok & 0.426 & 0.468 & 0.489 & 0.35 & 0.480 & 0.7 & 77 \\
& bad & 0.5 & 0.690 & 0.883 & 0.25 & 0.660 & 0.95 & 11,441 \\
\hline$f_{2} \aleph_{1}^{\ell_{\text {out }}}$ & good & 0.319 & 0.390 & 0.415 & 0.35 & 0.428 & 0.55 & 14 \\
$\mathrm{NN}: 9-2-1$ & ok & 0.426 & 0.470 & 0.489 & 0.25 & 0.477 & 0.6 & 1,215 \\
& bad & 0.5 & 0.589 & 0.840 & 0.3 & 0.558 & 0.8 & 10,291 \\
\hline$f_{1} \aleph_{>1}^{\ell_{\text {out }}}$ & good & 0.181 & 0.360 & 0.415 & 0.15 & 0.353 & 0.6 & 796 \\
$\mathrm{NN}: 9-2-5$ & ok & 0.426 & 0.459 & 0.489 & 0.25 & 0.441 & 0.65 & 1,794 \\
& bad & 0.5 & 0.597 & 0.862 & 0.25 & 0.556 & 0.8 & 8,930 \\
\hline$f_{2} \aleph_{>1}^{\ell_{\text {out }}}$ & good & 0.223 & 0.344 & 0.404 & 0.2 & 0.365 & 0.55 & 13 \\
$\mathrm{NN}: 9-2-5$ & ok & 0.426 & 0.461 & 0.489 & 0.4 & 0.464 & 0.65 & 276 \\
& bad & 0.5 & 0.649 & 1 & 0.35 & 0.623 & 1 & 11,231 \\
\hline \hline
\end{tabular}

\begin{tabular}{lc|rrr|rrr|r}
\hline \hline \multicolumn{8}{c|}{ If $\mathcal{E}_{C_{N}}^{T r}<0.489$ then good. If $\mathcal{E}_{C_{N}}^{T r}<0.52$ then } & ok. Otherwise bad. \\
& Class & $\left\lfloor\mathcal{E}_{C_{N}}^{T r}\right\rfloor$ & $\overline{\mathcal{E}_{C_{N}}^{T r}}$ & $\left\lceil\mathcal{E}_{C_{N}}^{T r}\right\rceil$ & $\left\lfloor\mathcal{E}_{C_{N}}^{T e}\right\rfloor$ & $\overline{\mathcal{E}_{C_{N}}^{T e}}$ & $\left\lceil\mathcal{E}_{C_{N}}^{T e}\right\rceil$ & Count \\
\hline$f_{1} \aleph_{1}^{\ell_{\text {out }}}$ & good & 0.489 & 0.489 & 0.489 & 0.423 & 0.423 & 0.423 & 1 \\
$\mathrm{NN}: 9-2-1$ & $o k$ & 0.503 & 0.512 & 0.518 & 0.240 & 0.506 & 0.74 & 4 \\
& bad & 0.520 & 0.709 & 0.913 & 0.328 & 0.674 & 0.960 & 11,515 \\
\hline$f_{2} \aleph_{1}^{\ell_{\text {out }}}$ & good & & & & & & & 0 \\
$\mathrm{NN}: 9-2-1$ & ok & 0.495 & 0.504 & 0.513 & 0.580 & 0.590 & 0.6 & 2 \\
& bad & 0.52 & 0.705 & 0.866 & 0.348 & 0.701 & 0.857 & 11,518 \\
\hline$f_{1} \aleph_{>1}^{\ell_{\text {out }}}$ & good & 0.218 & 0.444 & 0.489 & 0.2 & 0.477 & 0.8 & 1,059 \\
$\mathrm{NN}: 9-2-5$ & ok & 0.489 & 0.506 & 0.520 & 0.317 & 0.505 & 0.823 & 698 \\
& bad & 0.52 & 0.638 & 0.889 & 0.32 & 0.620 & 0.846 & 9,763 \\
\hline$f_{2} \aleph_{>1}^{\ell_{\text {out }}}$ & good & 0.4 & 0.449 & 0.489 & 0.4 & 0.484 & 0.6 & 62 \\
$\mathrm{NN}: 9-2-5$ & ok & 0.489 & 0.505 & 0.518 & 0.44 & 0.552 & 0.743 & 40 \\
& bad & 0.52 & 0.705 & 1 & 0.44 & 0.704 & 1 & 11,418 \\
\hline
\end{tabular}




\subsection{A Brief Empirical Comparison to Other Techniques}

\subsubsection{NN-GEP vs. a Classical Neural Network}

A neural network backpropagation algorithm [Anderson, 2008] was run 12 times with logistic activation functions and using the training and testing data sets for the Insunza problem NN:5-1-1. After 1,000 epochs, the networks were terminated. Fig. 7.12 on page 141 shows the best 5 solutions from the 12 runs. The minimum training error is $\mathcal{E}_{C}^{T r}=0.1937$ and the ninimum testing error for a different neural network is $\mathcal{E}_{C}^{T e}=0.2139$.

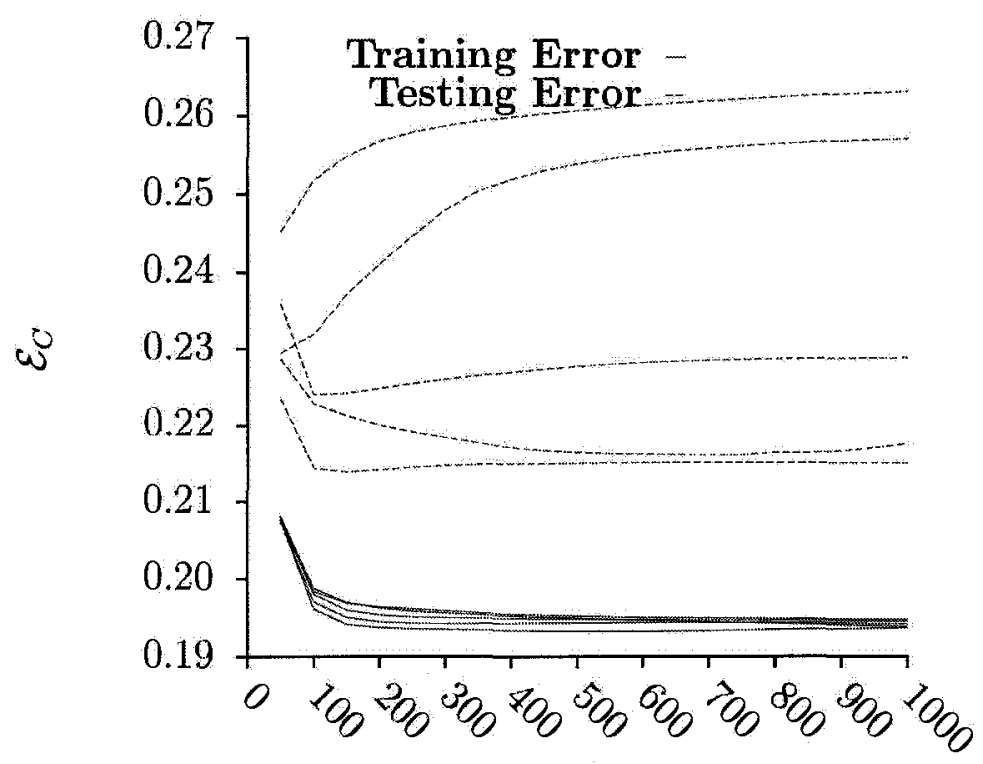

Epoch

Figure 7.12: Results for best 5 classically trained networks on Insunza data. The minimum training error for the best network out of 12 is 0.1937 . The minimum testing error for the best network (not the same network as the minimum for training error) is 0.2139

For NN-GEP, the classical error measure $\mathcal{E}_{C}$ and the neuron output mapping function ${ }_{f_{1}} \aleph_{>1}^{\ell_{\text {out }}}$ were selected to compare against the classical result. They were selected because Table 7.5 on page 135 indicates that this mapping function is one of the best. 
The experimental database corresponding to this function has 11,520 results. The results were searched in order to find experiments that resulted in network solutions that had lower training error $\left(\mathcal{E}_{C}^{T r}<0.1937\right)$ and lower testing error $\left(\mathcal{E}_{C}^{T e}<0.2139\right)$ in order to see how many solutions are better than the best training and best testing result over the series of 12 runs, which does not correspond to an actual neural network. The query resulted in 11,465 actual neural network results. This means that $\frac{11465}{11520} \cdot 100=99.52 \%$ of NN-GEP's results are better than the 12 classical neural network results considered all at once. Put another way, 56 out of 11,520 experiments were worse. In other words, setting NN-GEP's controlling parameters to almost any of the investigated values (See Table 6.6 on page 104) resulted in a solution that was better than all of the 12 classical neural network results considered simultaneously.

In terms of a specific result, the best solution found by NN-GEP after 497 generations ${ }^{7}$ using the classical error measure $\mathcal{E}_{C}$ and the neuron output mapping function ${ }_{f_{1}} \aleph_{>1}^{\mathcal{E}_{\text {out }}}$, is experiment $\operatorname{Exp}_{15,097}^{\mathcal{E}_{C}, \mathrm{NN}: 5-1-2}$ that has training error $\mathcal{E}_{C}^{T r}=0.043$ and testing error $\mathcal{E}_{C}^{T e}=0.049$, which is about $\frac{0.1937}{0.043} \approx 4.5$ times better training error and about $\frac{0.2139}{0.049} \approx 4.36$ times better testing error than the combined best of the 12 classically trained networks, which doesn't correspond to any particular network.

In addition, Breast Cancer was also used for computing 12 classical ANNs. The minimum training error was found to be $\mathcal{E}_{C}^{T r}=0.134$ while the minimum testing error was found to be $\mathcal{E}_{C}^{T e}=0.082$. A search of the experimental results for $\mathcal{E}_{C}$ and ${ }_{f_{1}} \aleph_{>1}^{\mathcal{e}_{\text {out }}}$ resulted in $\frac{9718}{11520} \cdot 100=84.36 \%$ that were less than two minimums found by the 12 classical ANNs.

\subsubsection{NN-GEP vs. k-nearest neighbours}

The k-nearest neighbours (knn) algorithm [Witten and Frank, 2005] was run on the complete 1,225 object Insunza data set for $k=3$ and the resulting confusion matrix in Table 7.8 on page 143 has an error of $\left(1-\frac{81+1091}{81+1091+40+13}=1-0.9567=0.043\right)$. Comparing this result to Table $\mathbf{7 . 5}$ on page 135 shows that NN-GEP has a best result on the training data that is the same error as knn on the complete data.

\footnotetext{
${ }^{7}$ Within the top 20 solutions, 1 solution required only 6 generations.
} 
Table 7.8: knn Confusion Matrix for all Insunza data $(k=3)$

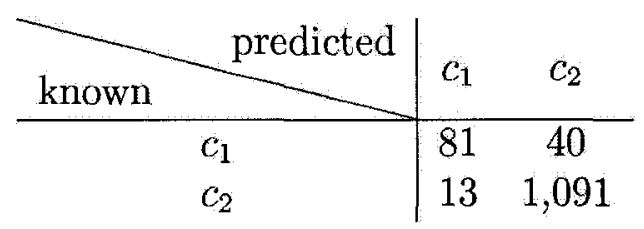

Table 7.9: j48 Confusion Matrix for all Insunza data

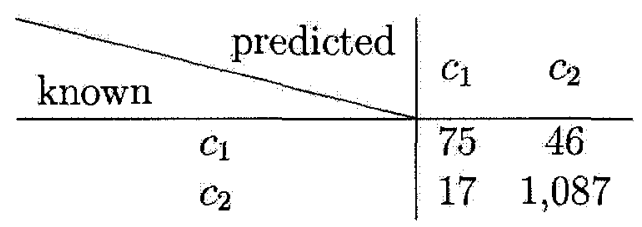

\subsubsection{NN-GEP vs. an Inductive Learner (e.g. decision tree)}

The J48 algorithm [Witten and Frank, 2005] was run on the complete 1,225 object Insunza data set by first discretizing the data into 5 equal intervals over each attribute and resulted in a tree with 37 nodes and the confusion matrix in Table 7.9 on page 143 has an error of $\left(1-\frac{75+1087}{75+1087+46+17}=1-0.9486=0.051\right)$. Comparing this result to Table 7.5 on page 135 shows that NN-GEP has a best result on the training data that is better than $\mathrm{J} 48$ on the complete data.

\subsubsection{NN-GEP vs. GEP with Multiple Chromosomes}

Breast Cancer results are reported in [Valdés et al., 2007] in which GEP experiments using 5 and 8 primitive functions were performed. This thesis uses 3 primitive functions (See Table 6.6 on page 104). The GEP result using 5 primitive functions is shown in Eq. 7.37 on page 143.

$$
\begin{aligned}
\varphi_{X}= & v 2+v 6+v 3+((v 3 / v 2)-v 3) \\
& +((v 6-(v 9+v 6)) / v 5) \\
\varphi_{Y}= & \left(v 7 /\left((v 1+v 4)+k_{y 1}\right)\right)+((v 7 * v 3) /(v 3+v 3)) \\
& +(v 4-v 7)+(v 7 /((v 2+v 3) * p o w(v 6, v 4)))+v 7 \\
\varphi_{Z}= & v 5+\left(v 6 /\left(k_{z 1} / v 9\right)\right)+((v 6+v 6) /(v 5+v 9)) \\
& +(v 8-v 5)+(v 1-v 6)
\end{aligned}
$$


where $k_{y 1}=3.73$, and $k_{z 1}=9.95$.

The best Breast cancer data experiment (No. 28) using 8 functions yielded the vector function mapping $(\varphi)$ in Eq. 7.38 on page 144:

$$
\begin{aligned}
\varphi_{X}= & v 3+\sin (\exp (v 6))+(((v 5 / v 5)+v 9) / v 8) \\
& +\cos \left(\left(\sin (v 7) / p o w\left(v 8, k_{x 1}\right)\right)\right)+v 6 \\
\varphi_{Y}= & k_{y 1}+\cos \left(\cos \left(\left(k_{y 2} * \cos (v 5)\right)\right)\right)+v 7+k_{y 3} \\
& +(v 4+(v 5 / v 6)) \\
\varphi_{Z}= & \sin (v 4)+v 1+k_{z 1}+v 8+\sin ((\sin (v 2) / v 9))
\end{aligned}
$$

where $k_{x 1}=8.53, k_{y 1}=\exp (1.50), k_{y 2}=9.39, k_{y 3}=\cos (7.06), k_{z 1}=4.79$.

\subsubsection{NN-GEP vs. Rough Sets}

Section 7.4.2 on page 146 and its published form [Barton and Valdés, 2008] discuss the results when comparing Rough Sets and NN-GEP. In summary, NN-GEP is able to find two relevant attributes, where a Rough Sets algorithm is only able to find one reduct containing one of the attributes, not both.

\subsubsection{NN-GEP vs. Individual Dichotomization}

Section 7.4.2 on page 146 and its published form [Barton and Valdés, 2008] discuss the results when comparing an individual dichotomization technique and NN-GEP. In summary, NN-GEP is able to find two relevant attributes, where the individual dichotomization algorithm (by design) is only able to find one of the attributes.

\subsubsection{NN-GEP vs. a Collection of Neural Networks}

Work on publicly available Breast Cancer [Brown, 2004] studies the issue of diversity in ensembles of neural networks. In particular, Fig. 7.13 on page 145 reproduces a figure from that work demonstrating results for different collections of neural networks but not for the single network case. That study has a minimum testing error rate of $\approx 0.028$. NN-GEP is a single neural network (not an ensemble) and in Table 7.6 on page 137 it can be seen that NN-GEP has one solution with a training error 

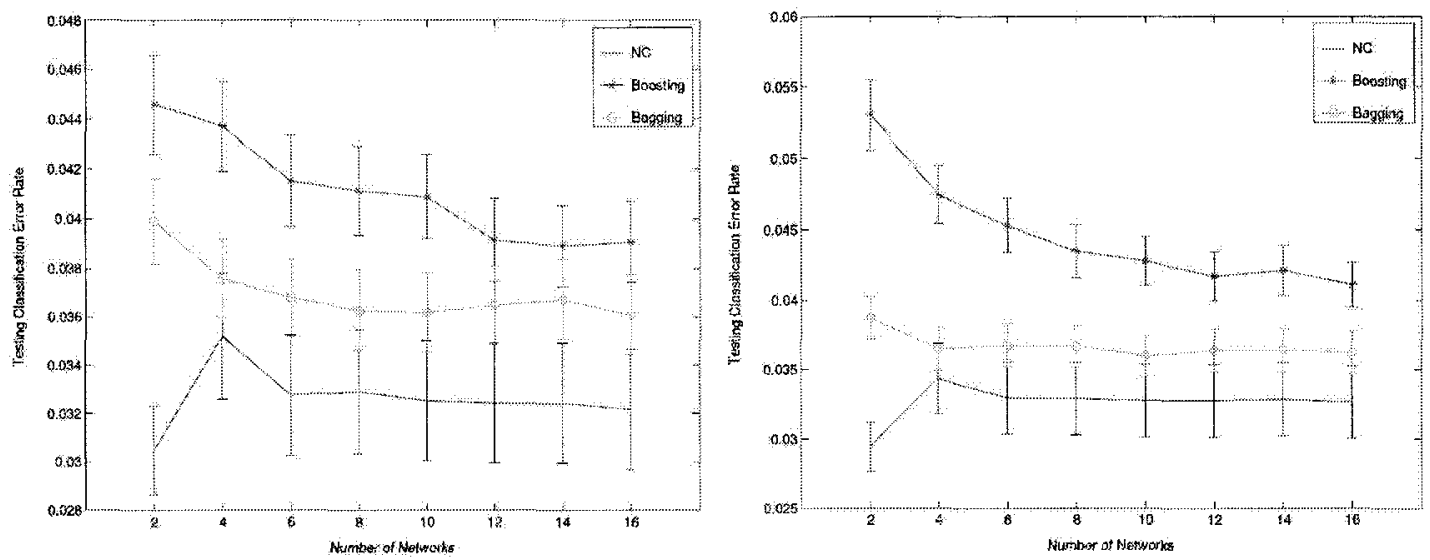

Figure 7.13: Results for collections of neural networks (from 2 to 16) on the breast cancer data set [Brown, 2004, p.87,89].

Left: 2 hidden nodes per network. Right: 6 hidden nodes per network.

$\mathcal{E}_{C}^{T r}=0.020$ and testing error $\mathcal{E}_{C}^{T e}=0$. Therefore, it can be concluded that NN-GEP is able to produce results that are at least as good, in terms of performance, as an ensemble of neural networks on a publicly available breast cancer data set.

\subsection{Published Magnetic Resonance Spectra $(c=4)$ Results}

The previously reported broad scale results have guided the preliminary experiments on Magnetic Resonance Spectra of Human Brain Cancers in which NN-GEP and Rough Sets were investigated, along with baseline performance (individual dichotomization) [Barton and Valdés, 2008].

\subsubsection{Published Rough sets Results}

The methodology is: i) the training set was discretized according to the global method described in [Bazan et al., 2000], [Bazan and M. S. Szczuka, 2002], ii) reducts (see Section 2.3.1.1 on page 36) were computed using both an exhaustive algorithm and a genetic algorithm [Bazan et al., 2000], [Wróblewski, 1995], iii) classification rules were generated from the reducts, iv) the test set was discretized using the same cuts produced by the discretization of the training set, and finally, $v$ ) the set was classified using the rules obtained for the training set.

Both reduct computation algorithms found a single reduct on the training set. 
Moreover, it was a simple reduct composed of a singleton attribute $\left(\left\{V_{270}\right\}\right)$. Both sets of classification rules consist of the common single rule:

$$
\text { IF } V_{270}\left\{\begin{array}{l}
\geq 69.374496 \Rightarrow C_{1} \text { (i.e. Normal) } \\
<69.374496 \Rightarrow C_{2} \text { (i.e. Diseased) }
\end{array}\right.
$$

which classifies the training set with $100 \%$ accuracy and when applied to the test set, it also classifies with $100 \%$ accuracy. This is very interesting, as it shows that a single attribute $\left(V_{270}\right.$, concentration $\approx 1.969 \mathrm{ppm}$ ) (out of the original 200 ) is capable of discriminating the spectra from normal cases from those of the malignant class.

\subsubsection{Published NN-GEP Results}

The experimental settings for the published results are reported in Table 6.4 on page 101. Two series of experiments, one of size 240, and the other size 2250 led to 26 explicit neural networks that, when interpreted as classifiers, had $100 \%$ training and $100 \%$ testing error; a very interesting preliminary result. In order to study the properties of these high performing solutions, the space constructed from the mapping function associated with each of the 26 networks is summarized in Fig. 7.14 on page 147. It can be seen that all 26 spaces (horizontal lines in Fig. 7.14 on page 147) perfectly separate the 2 classes and that the 26 solutions can be divided into 4 equivalence classes based on constructed space magnitude: i) extra large magnitude [-150000, 200000] (1 solution), ii) large magnitude [-4000,6000] (14 solutions), iii) medium magnitude $[-1000,2000]$ (2 solutions), and $i v$ ) small magnitude $[-200,100]$ (9 solutions); with the small magnitude solutions lying closest to the magnitude of the training and testing data.

The 26 spaces shown in Fig. 7.14 on page 147 may also be analyzed in terms of their associated mapping functions. In particular, the 26 equations contain only 50 of the 200 attributes present within the input data; with 43 attributes occurring in exactly one equation, 3 attributes occurring in two equations and 2 attributes occurring in exactly three equations. The two most frequent attributes are $V_{270}$ occurring in exactly eleven equations and $V_{271}$ occurring most frequently, and in sixteen equations. In addition, it is observed that $V_{271}$ was more frequently used than 


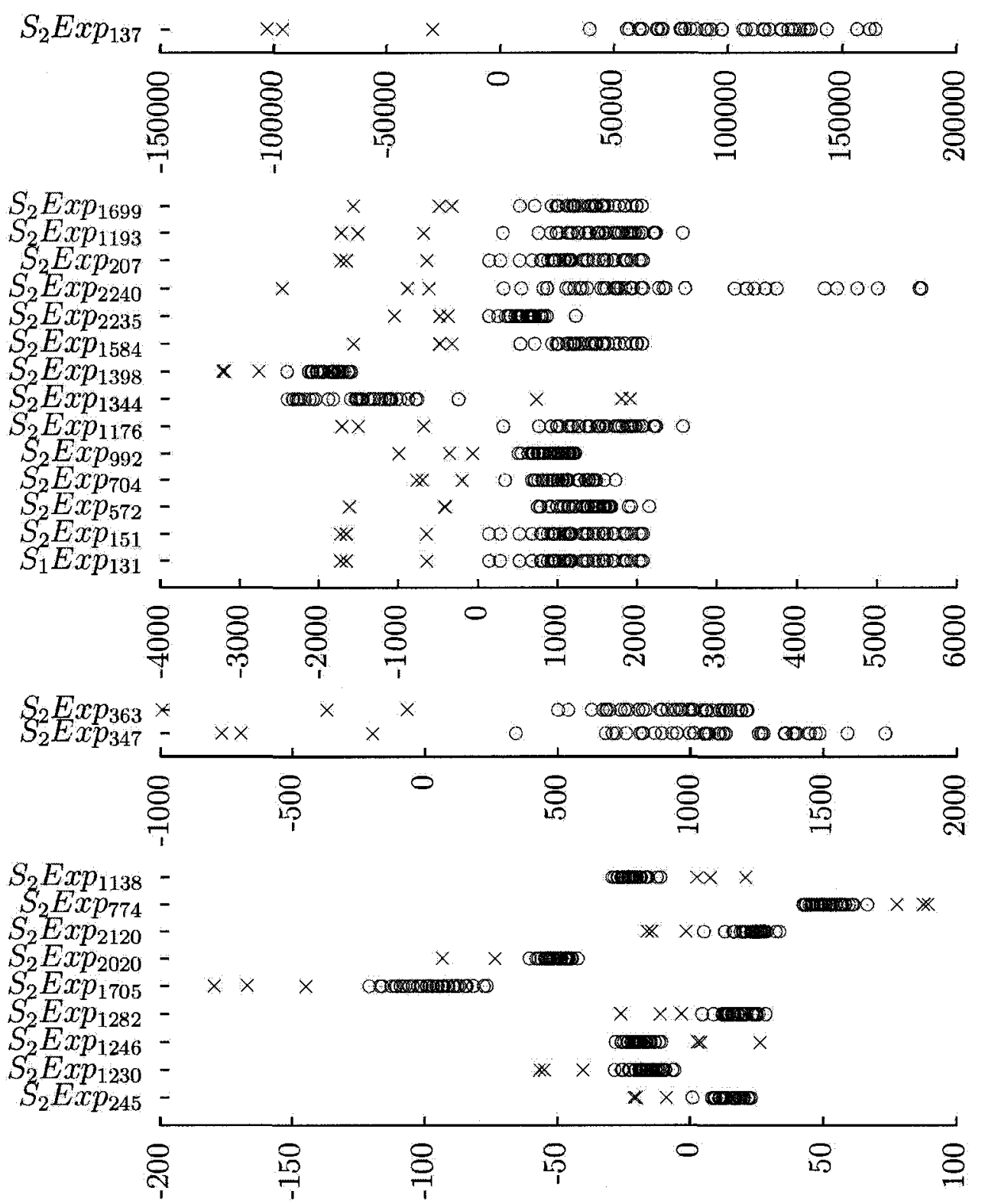

Figure 7.14: Best 26 mapped 1D spaces (varying orders of magnitude) from nonlinear discriminant analysis of neural network (NN-GEP) solutions having 200 input variables for published MRS data. All 26 spaces have an associated explicit classifier (not shown) with 0.00 training and validation error. $X$ 's correspond to testing objects belonging to healthy class. $O$ 's correspond to diseased patient samples.

$V_{270}$ within good solution networks and that it was not discovered by the Rough Sets approaches that were investigated, which only discovered attribute $V_{270}$.

Of the 26 good solution results (100\% training and 100\% testing accuracy), 3 are 
now highlighted that show use of the 2 most frequent variables (as both independent and joint usage) in the mapping and classifier results. It can be observed from Fig. 7.14 on page 147, that the mapping results may be converted into the good classifiers through rescaling (and possibly reflection about a point) of the constructed spaces.

An example NDA and classifier result involving $V_{270}$ was discovered in experiment $\mathrm{S}_{2} \operatorname{Exp}_{207}$ and resulted in the construction of a $200 \mathrm{D}$ to $1 \mathrm{D}$ mapping function $\varphi_{1}(\cdot)=$ $66.86-V_{270}$ and the following classifier (with $100 \%$ training and testing accuracy):

$$
\operatorname{IF}\left(66.86-V_{270}\right)^{3}\left\{\begin{aligned}
< & 0.5 \Rightarrow C_{1} \text { (i.e. Normal) } \\
= & 0.5 \Rightarrow \text { Undecidable } \\
> & 0.5 \Rightarrow C_{2} \text { (i.e. Diseased) }
\end{aligned}\right.
$$

An example NDA and classifier result involving $V_{271}$ was discovered in experiment $S_{2} \operatorname{Exp}_{347}$ and resulted in the construction of a $200 \mathrm{D}$ to $1 \mathrm{D}$ mapping function $\varphi_{1}(\cdot)=V_{271}-V_{234}-27.69$ and the following classifier (with $100 \%$ training and testing accuracy):

$$
\text { IF }-28.75\left(V_{271}-V_{234}-27.69\right)-50.78\left\{\begin{aligned}
<0.5 & \Rightarrow C_{1} \text { (i.e. Normal) } \\
=0.5 & \Rightarrow \text { Undecidable } \\
>0.5 & \Rightarrow C_{2} \text { (i.e. Diseased) }
\end{aligned}\right.
$$

An example NDA and classifier result involving both $V_{270}$ and $V_{271}$ was discovered in experiment $S_{2} \operatorname{Exp}_{1699}$ and resulted in the construction of a $200 \mathrm{D}$ to $1 \mathrm{D}$ mapping function $\varphi_{1}(\cdot)=V_{331}-V_{295}-V_{271}-V_{270}-V_{195}+V_{179}$ and the following classifier (with $100 \%$ training and testing accuracy):

IF $V_{331}-V_{295}-V_{271}-V_{270}-V_{195}+V_{179}+137.40\left\{\begin{aligned}<0.5 & \Rightarrow C_{1} \text { (i.e. Normal) } \\ =0.5 & \Rightarrow \text { Undecidable } \\ >0.5 & \Rightarrow C_{2} \text { (i.e. Diseased) }\end{aligned}\right.$

\subsubsection{Published Individual Dichotomization (Baseline) Results}

A systematic exploration of each single attribute in the training set was made with the individual dichotomization algorithm (see Section 2.3.2 on page 37). The probability 


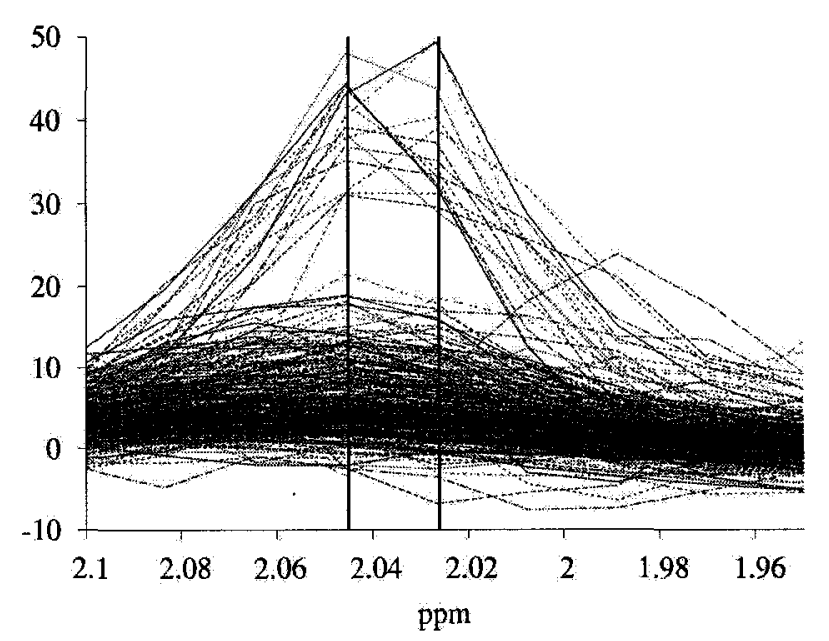

Figure 7.15: All patient samples (285 MRS spectra) for a subset of the variables, of which two $\left(V_{270}\right.$ and $V_{271}$ ) out of 200 may possibly be used (independently or jointly) for discrimination between normal and diseased samples. The larger valued concentrations at the two indicated locations $\left([31.075169 . .48 .118134]\right.$ for $V_{270}$ and $[29.067427 . .49 .497776]$ for $\left.V_{271}\right)$ are normal samples.

threshold was set to $1\left(p_{T}=1\right)$ in order to find the highest conditional probabilities of the classes given the attribute dichotomization. It was found that $P$ (class $=$ normal $\left./\left(V_{270} \geq 69.375\right)\right)=1$ and that $P\left(\right.$ class $=$ normal $\left./\left(V_{271} \geq 68.257\right)\right)=1$. When these probabilities are computed on the test set using the same conditionals, the result was the same, showing that both $V_{270}$ and $V_{271}$ (spectral peaks at 1.969 and 1.95 pprn respectively), can individually discriminate the normal from the malignant cases, thus confirming the results found with rough sets and especially with the NN-GEP network. Rough sets found $V_{270}$ but not $V_{271}$, whereas NN-GEP detected $V_{270}$ and $V_{271}$ as the two most important attributes, confirmed by individual dichotomization. 


\subsection{Chapter Summary}

Results for NN-GEP have been reported on 4 data sets; 3 of which were amenable to broad scale experimentation. A comparison with a number of techniques has been done with NN-GEP showing promise to be as least as good (See Table 7.10 on page 150). A large number (99.52\%) of the Insunza results are better than a hypothetical classically trained ANN indicating that NN-GEP is both able to find a large number of low error skewed results and able to find a result that is low enough (as indicated by both the knn result and the underground cave prediction for measuring stations over an unknown location). A particular mapping function $\left(f_{1} \aleph_{>1}^{\ell_{\text {out }}}\right)$ was demonstrated to consistently perform well and produce good solutions that are distributed throughout the parameter space. In addition, results have been published for a real world data set that learned the neuron functions for a NN:200-1-1 neural network. Next, a summary of all of the main points put forth in the thesis shall be made, including possible avenues for future research.

Table 7.10: Summary of comparison to other techniques for ${ }_{f_{1}} \aleph_{>1}^{\ell_{\text {out }}}$ and $\mathcal{E}_{C}$.

\begin{tabular}{l|l|l}
\hline \hline Technique & Technique's Result & NN-GEP's Result \\
\hline \hline $\begin{array}{l}\text { Classical ANN on In- } \\
\text { sunza }(12 \text { trials) }\end{array}$ & $\begin{array}{l}\text { Min. } \mathcal{E}_{C}^{T r}=0.1937, \\
\text { Min. } \mathcal{E}_{C}^{T e}=0.2139\end{array}$ & $\frac{11465}{11520} \cdot 100=99.52 \%$ \\
\hline $\begin{array}{l}\text { knn }(k=3) \text { on All In- } \\
\text { sunza }\end{array}$ & $\mathcal{E}_{C}=0.043$ & min. $\mathcal{E}_{C}^{T r}$ is equal \\
\hline $\begin{array}{l}\text { J48 algorithm on All In- } \\
\text { sunza }\end{array}$ & $\begin{array}{l}\mathcal{E}_{C}=0.051 \text { using } 5 \text { equal } \\
\text { intervals }\end{array}$ & min. $\mathcal{E}_{C}^{T r}$ is better \\
\hline $\begin{array}{l}\text { Classical ANN on Breast } \\
\text { Cancer (12 trials) }\end{array}$ & $\begin{array}{l}\text { Min. } \mathcal{E}_{C}^{T r}=0.134, \\
\text { Min. } \mathcal{E}_{C}^{T e}=0.082\end{array}$ & $\frac{9718}{11520} \cdot 100=84.36 \%$ \\
\hline $\begin{array}{l}\text { Collection of Neural Net- } \\
\text { works on Breast Cancer }\end{array}$ & Min. $\mathcal{E}_{C}^{T e} \approx 0.028$ & $\begin{array}{l}\text { Single FFNN, not ensem- } \\
\text { ble. } \mathcal{E}_{C}^{T r}=0.020, \mathcal{E}_{C}^{T e}=0\end{array}$ \\
\hline $\begin{array}{l}\text { Published Results for } \\
\text { Rough Sets on MRS }\end{array}$ & $\begin{array}{l}1 \text { reduct containing } 1 \text { at- } \\
\text { tribute (not both) }\end{array}$ & both attributes \\
\hline \hline
\end{tabular}




\section{Chapter 8}

\section{Summary of work, Conclusions and Future Work}

Science does not promise absolute truth, nor does it consider that such a thing necessarily exists. Science does not even promise that everything in the universe is amenable to the scientific process. Isaac Asimov. 1920-1992

All of the details for the results that have been obtained within this thesis were reported in the previous chapter and are summarized within this chapter. The main thesis conclusions are stated that may be inferred from the results. This chapter then suggests possible avenues for future research.

\subsection{Summary of Thesis Results}

A small subset of only the best performing results have been reported. An attempt was made to find the least complex equations within the experimental databases that contain the fewest variables and constants. For a precise description of how this was performed, see Section 6.2.5 on page 107 .

For the broad scale experiments, the following results were obtained:

1. Table 7.1 on page 110 indicates the neural network output vector mapping functions (See Section 4.2.5 on page 66) are, in order of best, to worst: $f_{1} \aleph_{>1}^{\aleph_{\text {out }}}(x), f_{2} \aleph_{1}^{\ell_{\text {out }}}(x), f_{2} \aleph_{>1}^{\ell_{\text {out }}}(x)$, and $f_{1} \aleph_{1}^{\ell_{\text {out }}}(x)$.

2. Table 7.1 on page 110 indicates that $\overline{\mathcal{E}^{T r}}$ for $\mathcal{E}_{C}$ is always less than $\overline{\mathcal{E}^{T r}}$ for $\mathcal{E}_{C_{N}}$. Similarly, $\overline{\mathcal{E}^{T e}}$ for $\mathcal{E}_{C}$ is always less than $\overline{\overline{\mathcal{E}}^{T e}}$ for $\mathcal{E}_{C_{N}}$.

3. Section 7.2 on page 134 discusses the parameter space for NN-GEP. The main conclusion is that for each of the 3 investigated data sets, good solutions are spread throughout the parameter space containing 10 parameters (See Table 6.6 on page 104). In other words, a global relation of parameter choice 
to neural network solution quality is not observable in Fig. $\mathbf{7 . 9}$ on page 136, Fig. 7.10 on page 138, and Fig. 7.11 on page 139 .

4. The following results were obtained that are more specialized to the data set:

(a) Insunza

Very good error distributions were obtained using $\mathcal{E}_{C}$, as can be seen in Fig. 7.1 on page 113.

Out of the 8 selected Insunza solutions, 4 indicate the potential location of an underground cave (confirmed by drilling a bore hole): Fig. 7.3 on page 118(a), Fig. 7.3 on page 118(d), Fig. 7.4 on page 119(b), and Fig. 7.4 on page 119(c).

The variables and their frequencies as reported in the 8 Insumza neural networks are: $v_{1}(5), v_{2}(8), v_{3}(8), v_{4}(4)$, and $v_{5}(6)$.

This indicates that $v_{2}$ and $v_{3}$ may be the most influential in terms of usefulness for good neural network solutions. Table 6.1 on page 92 describes all of the variables.

(b) Breast Cancer

The simplest neural network is Eq. 7.21 on page 126 that contains one variable $\left(v_{6}\right)$. Table 6.2 on page 94 describes all of the variables. For [Brown, 2004]'s work, a more complex solution involving an ensemble of neural networks was reported (See Section 7.3 on page 141).

The variables and their frequencies as reported in the 8 Breast Cancer neural networks are: $v_{1}(6), v_{2}(7), v_{3}(5), v_{4}(0), v_{5}(1), v_{6}(7), v_{7}(1)$, $v_{8}(3)$, and $v_{9}(0)$.

This indicates that $v_{1}, v_{2}, v_{3}$, and $v_{6}$ may be the most influential in terms of usefulness for good neural network solutions. Table 6.2 on page 94 describes all of the variables.

(c) Werenskiold

${ }_{f_{1}} \aleph_{>1}^{\ell_{\text {out }}}$ was able to produce networks with low errors (See Table 7.7 on page 140) for this multiple class learning problem. 
The variables and their frequencies as reported in the 2 best Werenskiold neural networks are: $v_{1}(1), v_{2}(2), v_{3}(0), v_{4}(2), v_{5}(2), v_{6}(0)$, $v_{7}(1), v_{8}(1)$, and $v_{9}(0)$.

This indicates that $v_{2}, v_{4}$ and $v_{5}$ may be the most influential in terms of usefulness for good neural network solutions. Table 6.3 on page 97 describes all of the variables.

This thesis has resulted in published work investigating human brain cancer magnetic resonance spectral data. In particular, two approaches, Rough Sets and NNGEP were investigated. Two out of 200 attributes were found to be most important (See Fig. 7.15 on page 149). Rough Sets found one; whereas the NN-GEP experiments found both. Confirmation was performed via a systematic Individual Dichotomization algorithm, which disregards attribute interactions; something that cannot (in general) be assumed a priori. Good preliminary results were obtained with $100 \%$ training and $100 \%$ testing accuracy that differentiate normal versus malignant samples (See Fig. $\mathbf{7 . 1 4}$ on page 147).

\subsection{Utility of the Approach}

NN-GEP has the weakness that a lot of controlling parameter are required. However, this is offset by the fact that almost any parameter setting is able to lead to lower errors than a classical neural network (See Section 7.2 on page 134 and Section 7.3.1 on page 141). The largest weakness that has been observed within the experimental results concerns the Werenskiold data. In that case, there are 5 classes that need to be discriminated. The broad scale experimental settings in Table 6.6 on page 104 show that only 3 primitive functions are used. Therefore, such a poor performance may be due to lack of appropriate nonlinearities available to the NN-GEP learning algorithm. It has also been observed that for Fig. 7.3 on page 118 and Fig. 7.4 on page 119 some neural networks seem to be learning the gradient of the cave location, rather than the actual cave location. Closer inspection of this result indicates that the equations are resulting in overflow. Further investigation is required to determine why it seems to only occur for one of the neural network output vector mapping 
function. It is also interesting to observe that for the 2 best Werenskiold neural network solutions in Section 7.1.3.1 on page 128, one of the output neurons is always unused.

\subsection{Thesis Contributions}

The contributions of this thesis (See Section 1.5 on page 7 ) are:

1. Development of hybrid neural network architecture for function approximation. This is reported in Fig. 4.4 on page 67, Section 2.2.5.1 on page 29, Section 2.2.5.2 on page 30 and Chapter 4 on page 62; in addition, Fig. 4.3 on page 66 and Fig. 2.9 on page 32 .

2. Development of a biologically-inspired representation for these neural networks. This is reported in Section 4.2 on page 63; in addition, Fig. 2.1 on page 13, and Fig. 2.2 on page 13.

3. Development of training algorithms for (1) and (2) based upon principles of Evolutionary Computation. This is reported in Section 2.2 on page 12; in particular, Alg. 1 on page 14, Section 2.2.2 on page 16, Section 2.2.3 on page 19, Section 2.2.4 on page 22, Section 2.2.5.1 on page 29, Section 2.2.5.2 on page 30 , and Section 2.2.5.3 on page 32 .

4. Experimental assessment of hybrid neural networks using real world data sets. This is reported in Chapter 7 on page 109; with the methodology reported in Chapter 6 on page 90 .

\subsection{Conclusions Drawn from Results}

This thesis makes the claim that "weights and activation functions do not need to be specified explicitly when learning the functions associated to neurons within a neural network, as is done in the classical case".

This claim is supported by published results in which NN-GEP was able to find a result that Rough Sets was not. This claim is also supported by the broad scale experiments for which good results were obtained with the Insunza and Breast Cancer 
data sets. This claim is also supported by a direct comparison to the minimum training and minimum testing errors found when computing 12 classical neural networks on the Insunza data. Results indicate that NN-GEP has lower errors $99.52 \%$ of the time.

\subsection{Potential Future Directions}

The best avenues for further research work are reported within this section.

A further investigation of the Werenskiold data needs to be made. Should other primitive functions be used? An open problem seems to be how to choose the set of primitive functions to investigate. One possibility is to allow the algorithm to learn which primitive functions to use. Or, perhaps, a periodic injection of new primitive functions (symbols) during the search process could occur. But which functions? And when should they be injected? Should functions with more non-linearity be added? That is, should injections follow a parsimonious approach in which more and more complex functions are inserted into the genome? Perhaps this could be thought of a a virus infecting the individual's genome. A related open question is should primitive functions be renoved from consideration? Additionally, Fereira's head strategy could be investigated. That is, what is a good policy for choosing functions within the head of a gene?

Further work needs to be performed in order to specify the neural network architecture. How are the number of layers chosen? How are the number of neurons per layer chosen? How are the number of genes per layer chosen? There are still many open questions regarding how to choose the parameters for NN-GEP. For example, are parameters that perform well on a given data set, also good on other data sets? Are there a core set of parameters that should always be used when searching for good neural network solutions?

Further work may be performed for hybridizing NN-GEP with other techniques. For example, classical optimization techniques in order to learn the constants within the neural network more precisely. Or, other strategies for construction of new constants could be made. For example, one possibility is to construct constants based on the data set under consideration. These training data derived constants could then 
be incorporated into the set of random constants that are currently considered. For example, the mean of an attribute could be one possible constant that could be used.

Another avenue for further research is to extend NN-GEP to use multiobjective algorithms. For example, each objective could be to minimize the error on a particular class. Then this set of non-dominated solutions might bypass the limit that was observed within the Werenskiold experiments. Other termination criteria could be investigated. In addition, cross-validation could be incorporated into the software.

In addition, it is noticed that NN-GEP contains a large number of configurable parameters. Interesting future work could be pursued in order to remove some of these parameters from the algorithm. One way to investigate this issue would be to determine the influence of each parameter and those that do not play a significant role in determination of good solutions could be calculated from within the algorithm.

The problem statement for this thesis reported that the learning problem also includes the fact that the FFNN is a general function approximator. This nonclassification work has not been demonstrated within this thesis but will be investigated within future work.

In general, the neural network solutions have a large variability with respect to their associated analytic functions across the parameter configurations. That is, for one set of 11,520 solutions with two output neurons, sorting on the function for one neuron results in very few duplicated functions. Future work should address this issue more closely. Is there less diversity than this preliminary result shows? Are duplicated functions associated to different neurons? That is, if neuron 1 and neuron 2 are swapped, would more duplicates appear? Also, what kind of diversity occurs during the evolutionary process? Is the population of neural networks very diverse throughout the entire evolutionary regine? 


\section{References}

[Alsultanny and Aqel, 2003] Alsultanny, Y. A. and Aqel, M. M. (2003). Pattern recognition using nultilayer neural-genetic algorithm. Neurocomputing, 51:237247. http://ww. sciencedirect.com/science/article/B6V10-469530K-3/2/ fc0d9df054eb100b185cc127249cc229 Accessed December 2008.

[Anderson, 2008] Anderson, C. W. (2008). C code for error backpropagation. http: //www.cs.colostate. edu/ anderson/code Accessed December 2008.

[Asuncion and Newman, 2007] Asuncion, A. and Newman, D. (2007). UCI machine learning repository. http://www.ics .uci.edu/ mlearn/MLRepository.html Accessed December 2008. Irvine, CA: University of California, School of Information and Computer Science.

[Bäck et al., 1997] Bäck, T., Fogel, D., and Michalewicz, Z. (1997). Handbook of Evolutionary Computation. Institute of Physics Publishing and Oxford Univ. Press, New York, Oxford.

[Bäck et al., 2000] Bäck, T., Fogel, D., and Michalewicz, Z. (2000). Evolutionary Computation 1 and 2. Institute of Physics Publishing, Bristol.

[Barnard, 1992] Barnard, E. (1992). Optimization for training neural nets. IEEE Transactions on Neural Networks, 3(2):232-240.

[Barton, 2004] Barton, A. J. (2004). Modelling variability in the leader algorithm family: A testable model and implementation. Technical Report NRC/ERB-1119. NRC 47429, National Research Council Canada, 1200 Montreal Road, Ottawa, Ontario.

[Barton and Valdés, 2008] Barton, A. J. and Valdés, J. J. (2008). Computational Intelligence Techniques Applied to Magnetic Resonance Spectroscopy Data of Human Brain Cancers. In Sixth International Conference on Rough Sets and Current Trends in Computing, number 5306 in Lecture Notes in Artificial Intelligence (LNAI) of the Lecture Notes in Computer Science (LNCS), pages 485-494. Springer-Verlag.

[Bazan et al., 2000] Bazan, J., Nguyen, H., Nguyen, S., Synak, P., and Wróblewski, J. (2000). Rough set algorithms in classification problem. In L. Polkowski, S. T. and Lin, T., editors, Rough Set Methods and Applications, pages 49-88, Heidelberg, New York. Physica-Verlag.

[Bazan et al., 1994] Bazan, J., Skowron, A., and Synak, P. (1994). Dynamic reducts as a tool for extracting laws from decision tables. In In: Proc. of the Symp. on 
Methodologies for Intelligent Systems. LNCS (LNAI), volume 869, pages 346-355. Springer, Heidelberg.

[Bazan and M. S. Szczuka, 2002] Bazan, J. G. and M. S. Szczuka, a. J. W. (2002). A new version of rough set exploration system. In Third. Int. Conf. on Rough Sets and Current Trends in Computing (RSCTC), LNCS 2475, pages 397-404, Springer-Verlag.

[Benediktsson et al., 1997] Benediktsson, J. A., Sveinsson, J. R., Ingimundarson, J. I., Sigurdsson, H. S., and Ersoy, O. K. (1997). Multistage classifiers optimized by neural networks and genetic algorithms. Nonlinear Analysis, 30(3):1323 1334. http://www.sciencedirect. com/science/article/B6VOY-3YCTK1X-56/ 2/d4024904e091d37665a21fae071395e8 Accessed December 2008.

[Bengio et al., 1994] Bengio, S., Bengio, Y., and Cloutier, J. (1994). Use of genetic programming for the search of a new learning rule for neural networks. Proceedings of the First IEEE Conference on Evolutionary Computation. IEEE World Congress on Computational Intelligence, 1:324-327.

[Block, 1962] Block, H. (1962). The perceptron: A model for brain functioning. Reviews of Modern Physics, 34(1):123-135.

[Borg and Lingoes, 1987] Borg, I. and Lingoes, J. (1987). Multidimensional similarity structure analysis. Springer-Verlag.

[Bornholdt and Graudenz, 1992] Bornholdt, S. and Graudenz, D. (1992). General asymmetric neural networks and structure design by genetic algorithms. Neural Networks, 5(2):327 - 334. http://www.sciencedirect.com/science/article/ B6T08-4HC1K76-D/2/fd484419295d6e05c69eaa35ddd31f5f Accessed December 2008 .

[Boute, 1992] Boute, R. T. (1992). The euclidean definition of the functions div and mod. ACM Transactions on Programming Languages and Systems, 14(2):127-144.

[Brameier and Banzhaf, 2001] Brameier, M. and Banzhaf, W. (2001). A comparison of linear genetic programming and neural networks in medical data mining. IEEE Transactions on Evolutionary Computation, 5(1):17-26.

[Brown, 2004] Brown, G. (2004). Diversity in Neural Network Ensembles. PhD thesis, University of Birmingham, Birmingham, United Kingdom.

[Bullinaria, 2005] Bullinaria, J. A. (2005). Evolving neural networks: is it really worth the effort? In In Proceedings of the European symposium on artificial neural networks, pages $267-272$.

[Burden, 1994] Burden, F. R. (1994). Mapping analytic functions using neural networks. Journal of Chemical Information and Computer Sciences, 34(6):1229-1231. 
[Carpenter and Barthelemy, 1994] Carpenter, W. C. and Barthelemy, J.-F. (1994). Common misconceptions about neural networks as approximators. Computing in Civil Engineering, 8(3):345-358.

[Chamberlin, 1890] Chamberlin, T. (1890). The method of multiple working hypotheses. Science, 15(366):92-97.

[Chandon and Pinson, 1981] Chandon, J. L. and Pinson, S. (1981). Analyse typologique. Théorie et applications. Masson, Paris.

[Chen et al., 2006] Chen, Y., Abraham, A., and Yang, B. (2006). Feature selection and classification using flexible neural tree. Neurocomputing, 70(1-3):305313. http://www.sciencedirect.com/science/article/B6V10-4K5JVR0-2/2/ 8c12b8d64904f4518f59df51828af1f1 Accessed December 2008.

[Cho and Shimohara, 1998] Cho, S.-B. and Shimohara, K. (1998). Evolutionary learning of modular neural networks with genetic programming. Applied Intelligence, 9(3):191-200.

[Chow and Chu, 1994] Chow, C. and Chu, C. (1994). On the configuration of multilayered feedforward networks by an evolutionary process. Proceedings of the 37th Midwest Symposium on Circuits and Systems, 1:531-534 vol.1.

[Colmenares and Perez, 1998] Colmenares, G. and Perez, R. (1998). A data reduction method to train, test, and validate neural networks. Proceedings of IEEE Southeastcon'98, pages 277-280.

[Cramer, 1985] Cramer, N. L. (1985). A representation for the adaptive generation of simple sequential programs. In Grefenstette, J. J., editor, Proceedings of an International Conference on Genetic Algorithms and the Applications, pages 183187, Carnegie-Mellon University, Pittsburgh, PA, USA. http://www.sover.net/ rnichael/nlc-publications/icga85/index.html Accessed December 2008.

[Crick, 1968] Crick, F. H. C. (1968). The origin of the genetic code. Journal of Molecular Biology, 38:367-379.

[Cun, 1988] Cun, Y. L. (1988). A theoretical framework for back-propagation. In Louretzky, D., G.Hinton, and Sejnowski, T., editors, Proceedings of the 1988 Connectionist Models Summer School, pages 21-28, CMU, Pittsburgh, Pa. Morgan Kaufmann.

[de Garis, 1990] de Garis, H. (1990). Genetic programming: building nanobrains with genetically programmed neural network modules. Neural Networks, 1990., 1990 IJCNN International Joint Conference on, pages 511-516 vol.3. 
[de Garis, 1991] de Garis, H. (1991). Gennets: genetically programmed neural netsusing the genetic algorithm to train neural nets whose inputs and/or outputs vary in time. Neural Networks, 1991. 1991 IEEE International Joint Conference on, pages 1391-1396 vol.2.

[de Garis, 1993] de Garis, H. (1993). Neurite networks: the genetic programming of cellular automata based neural nets which grow. Neural Networks, 1993. IJCNN '93-Nagoya. Proceedings of 1993 International Joint Conference on, 3:2921-2924 vol.3.

[Devos, 2005] Devos, A. (2005). Quantification and classification of magnetic resonance spectroscopy data and applications to brain tumour recognition. Technical Report Technical Report U.D.C. 616.831-073, Katholieke Universiteit Leuven, Dept. of Electronics.

[dos Santos Caldas de Matos et al., 2007] dos Santos Caldas de Matos, A. M. R., Suzuki, R., and Arita, T. (2007). Heterochrony and evolvability in neural network development. Artificial Life and Robotics, 11(2):175-182. doi:10.1007/s10015-007$0425-0$.

[Drchal and Snorek, 2008] Drchal, J. and Snorek, M. (2008). Tree-based indirect encodings for evolutionary development of neural networks. Artificial Neural Networks - ICANN 2008, 18th International Conference Proceedings, 2:839-848.

[Eiben and Jelasity, 2002] Eiben, A. and Jelasity, M. (2002). A critical note on experimental research methodology in ec. Proceedings of the 2002 Congress on Evolutionary Computation, 1:582-587.

[Eldredge and Gould, 1972] Eldredge, N. and Gould, S. J. (1972). Punctuated equilibria: an alternative to phyletic gradualism. Models in Paleobiology, pages 82-115.

[Eves, 1997] Eves, H. (1997). Foundations and Fundamental Concepts of Mathematics. Dover Publications Inc.,, 31 East 2nd Street, Mineola, N.Y. 11501, third edition. ISBN 0-486-69609-X.

[Fagundo et al., 1996] Fagundo, J., Valdés, J., and Rodríguez, J. (1996). Karst hydrochemistry (in spanish). In Research Group of Water Resources and Environmental Geology, University of Granada, Spain.

[Fagundo et al., 1990] Fagundo, J., Valdés, J. J., and Pulina, M. (1990). Hydrochemical investigations in extreme climatic areas, cuba and spitzbergen. In Water Resources Management and Protection in Tropical Climates, pages 45-54, Stockholm.

[Ferentinos, 2005] Ferentinos, K. P. (2005). Biological engineering applications of feedforward neural networks designed and parameterized by genetic algorithms. 
Neural Networks, 18(7):934 - 950. http://www.sciencedirect.com/science/ article/B6T08-4GDBT9J-4/2/9c6353c9420994f3ef05f97651877feb Accessed December 2008.

[Ferreira, 2001] Ferreira, C. (2001). Gene expression programming: A new adaptive algorithm for solving problems. Complex Systems, 13(2):87-129.

[Ferreira, 2004] Ferreira, C. (2004). Designing neural networks using gene expression programming. 9th Online World Conference on Soft Computing in Industrial Applications. Online proceedings no longer available. Paper obtained from http://www.gene-expression-programming . com/Author . asp Accessed December 2008 .

[Ferreira, 2006a] Ferreira, C. (2006a). Automatically defined functions in gene expression programming. In Nedjah, N., Abraham, A., and de Macedo Mourelle, L., editors, Genetic Systems Programming: Theory and Experiences, volume 13 of Studies in Computational Intelligence, pages 21-56. Springer, Germany. ISBN 3-540-29849-5.

[Ferreira, 2006b] Ferreira, C. (2006b). Gene Expression Programming. Springer, second edition. ISBN-10 3-540-32796-7.

[Ferri et al., 2009] Ferri, C., Hernndez-Orallo, J., and Modroiu, R. (2009). An experimental comparison of performance measures for classification. Pattern Recognition Letters, 30(1):27 - 38. http://www.sciencedirect.com/science/article/ B6V15-4TBXG8M-1/2/546ed14c769e933ed74f6d107686d9f7 Accessed December 2008.

[Fodstad, 2001] Fodstad, H. (2001). The Neuron Theory. Stereotactic and Functional Neurosurgery, 77:20-24.

[Funahashi, 1989] Funahashi, K.-I. (1989). On the approximate realization of continuous mappings by neural networks. Neural Networks, 2:183-192.

[Gallant, 1990] Gallant, S. I. (1990). Perceptron-based learning algorithms. IEEE Transactions on Neural Networks, 1(2):179-191.

[García-Pedrajas et al., 2006] García-Pedrajas, N., Ortiz-Boyer, D., and HervásMartínez, C. (2006). An alternative approach for neural network evolution with a genetic algorithm: Crossover by combinatorial optimization. Neural Networks, 19(4):514 - 528. http://www.sciencedirect.com/science/article/ B6T08-4HTCTJR-4/2/b4f7383c2f65591f1e30d2beb0db6d7f Accessed December 2008.

[Grönroos, 1999] Grönroos, M. (1999). A comparison of some methods for evolving neural networks. In Banzhaf, I. W., Daida, J., Eiben, A. E., Garzon, M. H., 
Honavar, V., Jakiela, M., and (eds.)., R. E. S., editors, GECCO-99: Proceedings of the Genetic and Evolutionary Computation Conference. July 13-17, 1999, Orlando, Florida USA, page 1442, San Francisco, CA. Morgan Kaufmann.

[Gros, 2003] Gros, C.-H. (2003). Genetic evolution of neural networks. In Koza, J. R., editor, Genetic Algorithms and Genetic Programming at Stanford 2003, pages 6874. Stanford Bookstore, Stanford, California, 94305-3079 USA.

[Gupta and Sexton, 1999] Gupta, J. N. D. and Sexton, R. S. (1999). Comparing backpropagation with a genetic algorithm for neural network training. Omega, 27(6):679 - 684. http://wWw.sciencedirect.com/science/article/B6VC4-3XP0H8T-7/ 2/08f771954904d3962211ae5dcc931252 Accessed December 2008.

[Hagan et al., 2002] Hagan, M. T., Demuth, H. B., and Beale, M. H. (2002). Neural Network Design. ISBN-10 0971732108.

[Hayes-Roth, 1975] Hayes-Roth, F. (1975). Adaptation in natural and artificial systems by John H. Holland. SIGART Newsletter, (53):15.

[Hecht-Nielsen, 2004] Hecht-Nielsen, R. (2004). Perceptrons. Technical Report 0403, UCSD Institute for Neural Computation, San Diego, California.

[Hinton and van Camp, 1993] Hinton, G. E. and van Camp, D. (1993). Keeping the neural networks simple by minimizing the description length of the weights. In COLT '93: Proceedings of the sixth annual conference on Computational learning theory, pages 5-13, New York, NY, USA. ACM.

[Holland, 1992] Holland, J. H. (1992). Adaptation in Natural and Artificial Systems: An Introductory Analysis with Applications to Biology, Control, and Artificial Intelligence. Number ISBN 0-262-58111-6. MIT Press.

[Hopfield, 1982] Hopfield, J. J. (1982). Neural networks and physical systems with emergent collective computational abilities. Proceedings of the National Academy of Sciences, 79(8):2554-2558.

[Hornik, 1989] Hornik, K. (1989). Multilayer Feedforward Networks are Universal Approximators. Neural Networks, 2:359-366.

[Hornik, 1993] Hornik, K. (1993). Some new results on neural network approximation. Neural Networks, 6(8):1069-1072.

[Howard and D'Angelo, 1995] Howard, L. and D'Angelo, D. (1995). The ga-p: a genetic algorithm and genetic programming hybrid. IEEE Expert, 10(3):11-15.

[Hu and Tsai, 2009] Hu, Y.-C. and Tsai; J.-F. (2009). Evaluating classification performances of single-layer perceptron with a choquet fuzzy integralbased neuron. Expert Systems with Applications, 36(2, Part 1):1793 - 
1800. http://www.sciencedirect.com/science/article/B6V03-4RDS1M4-7/2/ 0384248fe09f8d0ea6cdf776d656e4fa Accessed December 2008.

[Huang et al., 2009] Huang, C.-Y., Chen, L.-H., Chen, Y.-L., and Chang, F. M. (2009). Evaluating the process of a genetic algorithm to improve the backpropagation network: A monte carlo study. Expert Systems with Applications, 36(2, Part 1):1459 - 1465. http://www. sciencedirect.com/science/article/ B6V03-4RBYCYY-1/2/5ae5a5fe74c9358e028a7d74a6cbeb29 Accessed December 2008.

[Irie and Miyake, 1988] Irie, B. and Miyake, S. (1988). Capabilities of three-layered perceptrons. IEEE International Conference on Neural Networks, pages 641-648 vol.1.

[Johansson et al., 2006] Johansson, U., Löfström, T., König, R., and Niklasson, L. (2006). Building neural network ensembles using genetic programming. International Joint Conference on Neural Networks, pages 1260-1265.

[Jong, 1988] Jong, K. D. (1988). Learning with genetic algorithms: An overview. Machine Learning, 3(2):121-138.

[Jorna, 1988] Jorna, R. (1988). A comparison of presentation and representation: linguistic and pictorial., pages 172-85. Human-Computer Interaction: Psychonomic Aspects. Springer-Verlag, Berlin, Germany.

[Juliá-Sapé et al., 2006] Juliá-Sapé, M., Acosta, D., Mier, M., Arús, C., and Watson, D. (2006). A multi-centre, web-accessible and quality control-checked database of in vivo $\mathrm{mr}$ spectra of brain tumour patients. Magn Reson Mater Phy, 19.

[Kahramanli and Allahverdi, 2009] Kahramanli, H. and Allahverdi, N. (2009). Rule extraction from trained adaptive neural networks using artificial immune systems. Expert Systems with Applications, 36(2, Part 1):1513 1522. http://www.sciencedirect.com/science/article/B6V03-4R98K46-6/2/ 55bc7cf0707c538456be7573a79a5039 Accessed December 2008.

[Kassahun et al., 2007] Kassahun, Y., Edgington, M., Metzen, J. H., Sommer, G., and Kirchner, F. (2007). A common genetic encoding for both direct and indirect encodings of networks. In Proceedings of the Genetic and Evolutionary Computation Conference, pages 1029-1036.

[Kay, 2001] Kay, L. E. (2001). From logical neurons to poetic embodiments of mind: Warren s. mcculloch's project in neuroscience. Science in Context, 14:591-614. doi:10.1017/S0269889701000266.

[Kinnebrock, 1994] Kinnebrock, W. (1994). Accelerating the standard backpropagation method using a genetic approach. Neurocomputing, 6(5-6):583 - 
588. http://www.sciencedirect.com/science/article/B6V10-48V24SF-8/2/ 597da21964141c200752c6ddb7ef1aea Accessed December 2008.

[Kitano, 1994] Kitano, H. (1994). Neurogenetic learning: an integrated method of designing and training neural networks using genetic algorithms. Physica $D$ : Nonlinear Phenomena, 75(1-3):225 - 238. http://www.sciencedirect.com/ science/article/B6TVK-46TY4NH-J/2/220c1dbd17cabc809629deee5654a77b Accessed December 2008.

[Koehn, 1996] Koehn, P. (1996). Genetic encoding strategies for neural networks. In Proceedings of Information Processing and Management of Uncertainty in Knowledge-Based Systems.

[Korolev, 2007] Korolev, L. (2007). On evolutionary algorithms, neural-network computations, and genetic programming. mathematical problems. Automation and Remote Control, 68(5):811-821.

[Koza, 1989] Koza, J. R. (1989). Hierarchical genetic algorithms operating on populations of computer programs. In $11^{\text {th }}$ International Joint Conference on Artificial Intelligence, San Mateo, CA.

[Koza, 1992] Koza, J. R. (1992). Genetic Programming: On the Programming of Computers by Means of Natural Selection. MIT Press. ISBN 0-262-11170-5.

[Koza, 1994] Koza, J. R. (1994). Introduction to genetic programming in Advances in Genetic Programming, chapter 2, pages 21-42. MIT Press, Cambridge, MA, USA.

[Koza, 1995a] Koza, J. R. (1995a). Evolving the architecture of a multi-part program in genetic programming using architecture-altering operations. In Evolutionary Programming IV: Proceedings of the Fourth Annual Conference on Evolutionary Programming, pages 695-717. The MIT Press.

[Koza, 1995b] Koza, J. R. (1995b). Survey of genetic algorithms and genetic programming. In In: Wescon 95: E2. Neural-Fuzzy Technologies and Its Applications, pages 589-594, San Francisco. IEEE.

[Koza et al., 1996] Koza, J. R., Andre, D., Iii, F. H. B., and Keane, M. A. (1996). Use of automatically defined functions and architecture-altering operations in automated circuit synthesis using genetic programming. In Stanford University, pages 28-31. MIT Press.

[Koza and Rice, 1991] Koza, J. R. and Rice, J. P. (1991). Genetic generation of both the weights and architecture for a neural network. International Joint Conference on Neural Networks, 2:397-404. 
[Krawiec, 2007] Krawiec, K. (2007). Generative learning of visual concepts using multiobjective genetic programming, Pattern Recognition Letters, 28(16):2385 2400. http://www.sciencedirect.com/science/article/B6V15-4PD4X8D-1/2/ 83c907f1e46304853cee0bbef23c2ac5 Accessed December 2008.

[Kruskal, 1964] Kruskal, J. (1964). Multidimensional scaling by optimizing goodness of fit to a nonmetric hypothesis. Psichometrika, 29:1-27.

[Kutschera and Niklas, 2004] Kutschera, U. and Niklas, K. J. (2004). The modern theory of biological evolution: an expanded synthesis. Naturwissenschaften, $91(6): 255-276$.

[Ladroue, 2004] Ladroue, C. L. C. (2004). Pattern Recognition Techniques for the Study of Magnetic Resonance Spectra of Brain Tumours. PhD thesis, St George's Hospital Medical School, London, England.

[Lapedes and Farber, 1988] Lapedes, A. and Farber, R. (1988). How neural nets work. IEEE Conference on Neural Information Processing Systems, pages 442456.

[Lu et al., 2004] Lu, Z., Szafron, D., Greiner, R., Lu, P., Wishart, D., Poulin, B., Anvik, J., Macdonell, C., and Eisner, R. (2004). Predicting subcellular localization of proteins using machine learned classifiers. Bioinformatics, 20(4):547-556.

[Luke et al., 2008] Luke, S., Panait, L., Balan, G., Paus, S., Skolicki, Z., Popovici, E., Sullivan, K., Harrison, J., Bassett, J., Hubley, R., and Chircop, A. (2008). Ecj: A java-based evolutionary computation research system. http://www.cs.gmu.edu/ -eclab/projects/ecj Accessed December 2008.

[Maass, 2000] Maass, W. (2000). On the computational power of winner-take-all. Neural Computing, 12(11):2519-35.

[Maddy, 2008] Maddy, P. (2008). How applied mathematics became pure. The Review of Symbolic Logic, 1(1):16-41.

[Malik, 1980] Malik, M. A. (1980). Historical and pedagogical aspects of the definition of function. International Journal of Mathematical Education in Science and Technology, 11(4):489-492.

[Mao and Jain, 1993] Mao, J. and Jain, A. (1993). Discriminant analysis neural networks. IEEE International Conference on Neural Networks, 1:300-305.

[Mao and Jain, 1995] Mao, J. and Jain, A. (1995). Artificial neural networks for feature extraction and multivariate data projection. IEEE Transactions on Neural Networks, 6(2):296-317. 
[Marwala, 2007] Marwala, T. (2007). Bayesian training of neural networks using genetic programming. Pattern Recognition Letters, 28(12):1452 1458. http://www.sciencedirect. com/science/article/B6V15-4NC38M7-5/2/ dee1daa1b7f713474289040a57125fd4 Accessed December 2008.

[Matsumoto and Tazaki, 1998] Matsumoto, N. and Tazaki, E. (1998). Emergence of learning rule in neural networks using genetic programming combined with decision trees. Systems, Man, and Cybernetics, 1998. 1998 IEEE International Conference on, 2:1801-1805 vol.2.

[Mazzarello, 1999] Mazzarello, P. (1999). A unifying concept: the history of cell theory. Nature Cell Biology, 1:E13-E15.

[McCullagh, 2002] McCullagh, P. (2002). What is a statistical model? The Annals of Statistics, 30(5):1225-1310.

[McCulloch and Pitts, 1943] McCulloch, W. S. and Pitts, W. (1943). A logical calculus of the ideas immanent in neurvous activity. Bulletin of Mathematical Biophysics, $5: 115-133$.

[Messer and Kittler, 1998] Messer, K. and Kittler, J. (1998). Choosing an optimal neural network size to aid a search through a large image database. In Carter, J. N. and Nixon, M. S., editors, Online Proceedings of the Ninth British Machine Vision Conference. http://www. comp. leeds . ac .uk/bmvc2008/proceedings/1998/ index.htm Accessed December 2008.

[Miesenbock and Rothman, 1997] Miesenbock, G. and Rothman, J. E. (1997). Patterns of synaptic activity in neural networks recorded by light emission from synaptolucins. Proceedings of the National Academy of Sciences Neurobiology, 94:34023407.

[Misra et al., 2008] Misra, B., Dehuri, S., Dash, P., and Panda, G. (2008). A reduced and comprehensible polynomial neural network for classification. Pattern Recognition Letters, 29(12):1705 - 1712. http://www.sciencedirect.com/ science/article/B6V15-4SG4HG2-1/2/a84f7a4b49c263c67a258702b32d4080 Accessed December 2008.

[Montana and Davis, 1989] Montana, D. J. and Davis, L. (1989). Training feedforward neural networks using genetic algorithms. In Proceedings of the International Joint Conference on Artificial Intelligence. http://vishnu.bbn.com/ people/dmontana.html Accessed December 2008.

[Moravec, 1998] Moravec, H. P. (1998). When will computer hardware match the human brain? Journal of Transhumanism, 1. 
[Muni et al., 2004] Muni, D., Pal, N., and Das, J. (2004). A novel approach to design classifiers using genetic programming. IEEE Transactions on Evolutionary Computation, 8(2):183-196.

[NCBI, 2008] NCBI (2008). A science primer by the national center for biotechnology information. http://www.ncbi.nlm.nih.gov/About/primer/genetics_ cell.html Accessed December.

[Novikoff, 1962] Novikoff, A. (1962). On convergence proofs for perceptrons. In Symposium on Mathematical Theory of Automata, pages 615-622. Polytechnic Institute of Brooklyn.

[Nowak and Ohtsuki, 2008] Nowak, M. A. and Ohtsuki, H. (2008). Prevolutionary dynamics and the origin of evolution. Proceedings of the National Academy of Sciences, (doi:10.1073/pnas.0806714105). (accessed online: ahead of print).

[N.Y.Nikolaev and Iba, 2003] N.Y.Nikolaev and Iba, H. (2003). Learning polynomial feedforward neural networks by genetic programming and backpropagation. IEEE Transactions on Neural Networks, 14(2):337-350.

[Olden and Jackson, 2002] Olden, J. and Jackson, D. (2002). Illuminating the black box: A randomization approach for understanding variable contributions in artificial neural networks. Ecological Modelling, 154(1-2):135-150.

[OMG, 2008] OMG (2008). Unified modeling language (uml) by the object management group (omg). http://www.uml org Accessed December 2008.

[Orr, 1991] Orr, H. A. (1991). A test of fisher's theory of dominance. Proceedings of the National Academy of Science: Evolution, 88:11413-11415.

[Orr, 2005] Orr, H. A. (2005). The Genetic Theory of Adaptation: A Brief History. Nature Reviews, 6:119-127.

[Oxnard, 2004] Oxnard, C. E. (2004). Brain Evolution: Mammals, Primates, Chimpanzees, and Humans. International Journal of Primatology, 25(5):1127-1158. DOI 10.1023/B:IJOP.0000043355.96393.8b.

[Pal and Bhandari, 1994] Pal, S. K. and Bhandari, D. (1994). Selection of optimal set of weights in a layered network using genetic algorithms. Information Sciences, 80(3-4):213 - 234. http://www.sciencedirect.com/science/article/ B6VOC-48MYKOK-S/2/75414b3871190e84247eecc733f75358 Accessed December 2008 .

[Palaniappan and Eswaran, 2009] Palaniappan, R. and Eswaran, C. (2009). Using genetic algorithm to select the presentation order of training patterns that improves simplified fuzzy artmap classification performance. Applied Soft Computing, 9(1):100 - 106. http://www.sciencedirect.com/science/article/ 
B6W86-4S32NH4-1/2/d6328a4a5fe69624f 9 faf 4e8df86685c Accessed December 2008.

[Pardalos et al., 2000] Pardalos, P. M., Romeijn, H. E., and Tuy, H. (2000). Recent developments and trends in global optimization. Journal of Computational and Applied Mathematics, 124:209-228.

[Pawlak, 1982] Pawlak, Z. (1982). Rough sets. International Journal of Parallel Programming, 11(5):341-356.

[Pawlak, 1991] Pawlak, Z. (1991). Rough sets: Theoretical aspects of reasoning about data. System Theory, Knowledge Engineering and Problem Solving, 9.

[Pearson, 1901] Pearson, K. (1901). On lines and planes of closest fit to systems of points in space. Philosophical Magazine, 2(11):559-572. S. 6.

[Poggio and Girosi, 1990] Poggio, T. and Girosi, F. (1990). Networks for approximation and learning. In Proceedings of IEEE 78, pages 1481-1497.

[Poli, 1996] Poli, R. (1996). Discovery of symbolic, neuro-symbolic and neural networks with parallel distributed genetic programming. Technical Report CSRP96-14, University of Birmingham, School of Computer Science. Presented at 3rd International Conference on Artificial Neural Networks and Genetic Algorithms, ICANNGA'97.

[Poli, 1997] Poli, R. (1997). Discovery of symbolic, neuro-symbolic and neural networks with parallel distributed genetic programming. In Smith, G. D., Steele, N. C., and Albrecht, R. F., editors, Artificial Neural Nets and Genetic Algorithms: Proceedings of the International Conference, ICANNGA97, University of East Anglia, Norwich, UK. Springer-Verlag. published in 1998, ISBN 3-211-83087-1.

[Poli et al., 2008] Poli, R., Langdon, W. B., McPhee, N. F., and Koza, J. R. (2008). A field guide to genetic programming. online.

[Press et al., 2002] Press, W. H., Teukolsky, S. A., Vetterling, W. T., and Flannery, B. P. (2002). Numerical Recipes in C. Cambridge University Press, second edition. ISBN 0-521-43108-5.

[Pujol and Poli, 1998] Pujol, J. C. F, and Poli, R. (1998). Evolving the topology and the weights of neural networks using a dual representation. Journal Applied Intelligence, 8(1):73-84.

[Radi and Poli, 1999] Radi, A. and Poli, R. (1999). Genetic programming discovers efficient learning rules for the hidden and output layers of feedforward neural networks. Genetic Programming, Lecture Notes in Computer Science, 1598:120-134. 
[Ritchie et al., 2003] Ritchie, M., White, B., Parker, J., Hahn, L., and Moore, J. (2003). Optimization of neural network architecture using genetic programming improves detection and modeling of gene-gene interactions in studies of human diseases. BMC Bioinformatics, 4(1):28.

[Rosenblatt, 1958] Rosenblatt, F. (1958). The perceptron: A probabilistic model for information storage and organization in the brain. Psychological Review, 65(6):386408.

[Rosenblatt, 1961] Rosenblatt, F. (1961). Principles of Neurodynamics: Perceptrons and the Theory of Brain Mechanisms. Spartan Books, Washington, D.C.

[Rowland, 2003] Rowland, J. J. (2003). Generalisation and model selection in supervised learning with evolutionary computation. Lecture Notes in Computer Science: Applications of Evolutionary Computing, pages 1269-1289.

[Sacks et al., 1989] Sacks, J., Welch, W. J., Mitchell, T. J., and Wynn, H. P. (1989). Design and analysis of computer experiments. Statistical Science, 4(4):409-435.

[Sammon, 1969] Sammon, J. W. (1969). A non-linear mapping for data structure analysis. IEEE Trans. Computers, C18:401-408.

[Saxén and Pettersson, 2006] Saxén, H. and Pettersson, F. (2006). Method for the selection of inputs and structure of feedforward neural networks. Computers and Chemical Engineering, 30(6-7):1038 - 1045. http://www.sciencedirect.com/ science/article/B6TFT-4JHMS1S-1/2/c5b5d8be61e0eade2106a86b90e26ec0 Accessed December 2008.

[Schaffer et al., 1992] Schaffer, J., Whitley, D., and Eshelman, L. (1992). Combinations of genetic algorithms and neural networks: a survey of the state of the art. In International Workshop on Combinations of Genetic Algorithms and Neural Networks (COGANN-92), pages 1-37, Briarcliff Manor, NY, USA. Philips Labs.

[Schenker and Agarwal, 1996] Schenker, B. and Agarwal, M. (1996). Crossvalidated structure selection for neural networks. Computers and Chemical Engineering, 20(2):175 - 186. http://www. sciencedirect.com/science/article/ B6TFT-3Y0Y78N-5/2/69c2c43766e93437176c5cda3e5abd15 Accessed December 2008.

[Sexton et al., 1998] Sexton, R. S., Dorsey, R. E., and Johnson, J. D. (1998). Toward global optimization of neural networks: A comparison of the genetic algorithm and backpropagation. Decision Support Systems, 22(2):171 185. http://www.sciencedirect. com/science/article/B6V8S-3T82SVR-5/2/ 18d6f cd227497ccb80d4aab06bb2b657 Accessed December 2008. 
[Sexton et al., 1999] Sexton, R. S., Dorsey, R. E., and Johnson, J. D. (1999). Optimization of neural networks: A comparative analysis of the genetic algorithm and simulated annealing. European Journal of Operational Research, 114(3):589601. http://www.sciencedirect. com/science/article/B6VCT-3W1PN05-C/2/ 2a1c9efb2ca2dca35b1cfd5c035ada88 Accessed December 2008.

[Sexton and Gupta, 2000] Sexton, R. S. and Gupta, J. N. D. (2000). Comparative evaluation of genetic algorithm and backpropagation for training neural networks. Information Sciences, 129(1-4):45 - 59. http://www.sciencedirect.com/ science/article/B6VOC-41TMTRS-4/2/68134a512f3778913b28f2000e29c47b Accessed December 2008.

[Siddique and Tokhi, 2001] Siddique, M. and Tokhi, M. (2001). Training neural networks: backpropagation vs. genetic algorithms. Neural Networks, 2001. Proceedings. IJCNN '01. International Joint Conference on, 4:2673-2678 vol.4.

[Soule and Heckendorn, 2002] Soule, T. and Heckendorn, R. B. (2002). An analysis of the causes of code growth in genetic programming. Genetic Programming and Evolvable Machines, 3(3):283-309.

[Specht, 1991] Specht, D. (1991). A general regression neural network. Neural Networks, IEEE Transactions on, 2(6):568-576.

[Specht, 1990] Specht, D. F. (1990). Probabilistic neural networks. Neural Networks, 3:109-118.

[Spinellis, 2003] Spinellis, D. (2003). On the declarative specification of models. IEEE Software, 20(2):94-96.

[Stanley, 2004] Stanley, K. O. (2004). Efficient Evolution of Neural Networks through Complexification. Phd dissertation, University of Texas at Austin, Austin, Texas, USA. http://www.cs .ucf .edu/ kstanley Accessed December 2008.

[Stigler, 2005] Stigler, S. (2005). Fisher in 1921. Statistical Science, 20(1):32-49.

[Tan and Chia, 2001] Tan, C. L. and Chia, H. W. K. (2001). Genetic construction of neural logic networks. Neural Networks, 2001. Proceedings. IJCNN '01. International Joint Conference on, 1:732-737 vol.1.

[Tate et al., 2006] Tate, A. R., Underwood, J., Acosta, D. M., Juliá-Sapé, M., Majo, C., Moreno-Torres, A., Howe, F. A., van der Graaf, M., Lefournier, V., Murphy, M. M., Loosemore, A., Ladroue, C., Wesseling, P., Bosson, J. L., n. as, M. E. C., Simonetti, A. W., Gajewicz, W., Calvar, J., Capdevila, A., Wilkins, P. R., Bell, B. A., Rémy, C., Heerschap, A., Watson, D., Griffiths, J. R., and Aruś, C. (2006). Development of a decision support system for diagnosis and grading of brain tumours using in vivo magnetic resonance single voxel spectra. NMR Biomed, 19. 
[Tresp et al., 1994] Tresp, V., Ahmad, S., and Neuneier, R. (1994). Training neural networks with deficient data. In In: J. Cowan, G. T. and (eds.), J. A., editors, Advances in Neural Information Processing Systems, volume 6, pages 128-135. Morgan Kaufmann.

[Tsaih, 2003] Tsaih, R. (2003). The evolution of internal representation. Mathematical and Computer Modelling, 38(3-4):339-350. http://www. sciencedirect.com/ science/article/B6VOV-4B82PFC-21/2/5f024bf354d617200441389cb96ff0da Accessed December 2008.

[Tsaih, 1998] Tsaih, R. R. (1998). An explanation of reasoning neural networks. Mathematical and Computer Modelling, 28(2):37 - 44. http: //www. sciencedirect. com/science/article/B6VOV-3TK6GHN-4/2/ fd858a992ec06d3322f2b3a71b7797c7 Accessed December 2008.

[Tsakonas et al., 2004] Tsakonas, A., Aggelis, V., Karkazis, I., and Dounias, G. (2004). An evolutionary system for neural logic networks using genetic programming and indirect encoding. Journal of Applied Logic, 2(3):349 379. http://www. sciencedirect. com/science/article/B758H-4C8P84V-1/2/ e66a004270eeee4e1c50fa3e09ddd003 Accessed December 2008.

[Tsoulos et al., 2005] Tsoulos, I., Gavrilis, D., and Glavas, E. (2005). Neural network construction using grammatical evolution. International Symposium on Signal Processing and Information Technology, 0:827-831.

[Tuan-Hao et al,, 2006] Tuan-Hao, H., McKay, R., Essam, D., and Hoai, N. X. (2006). Solving symbolic regression problems using incremental evaluation in genetic programming. IEEE Congress on Evolutionary Computation, pages 2134-2141.

[Tukey, 1977] Tukey, J. W. (1977). Exploratory Data Analysis. Addison-Wesley, Reading, MA. ISBN 9780201076165.

[Valdés, 2002] Valdés, J. J. (2002). Virtual reality representation of relational systems and decision rules:. In Hajek, P., editor, Theory and Application of Relational Structures as Knowledge Instruments, Prague. Meeting of the COST Action 274.

[Valdés, 2003] Valdés, J. J. (2003). Virtual reality representation of information systems and decision rules:. In Lecture Notes in Artificial Intelligence, volume 2639 of $L N A I$, pages $615-618$. Springer-Verlag.

[Valdés and Barton, 2006] Valdés, J. J. and Barton, A. J. (2006). Virtual reality spaces for visual data mining with multiobjective evolutionary optimization: Implicit and explicit function representations mixing unsupervised and supervised properties. In IEEE Congress of Evolutionary Computation (CEC 2006), pages 5592-5598, Vancouver. IEEE. 
[Valdés and Barton, 2006] Valdés, J. J. and Barton, A. J. (2006). Virtual reality visual data mining via neural networks obtained from multi-objective evolutionary optimization: Application to geophysical prospecting. In IEEE International Joint Conference on Neural Networks (IJCNN), number NRC 48504, pages 9916-9923.

[Valdés and Barton, 2007] Valdés, J. J. and Barton, A. J. (2007). Multi-objective evolutionary optimization of neural networks for virtual reality visual data mining: Application to hydrochemistry. In IEEE International Joint Conference on Neural Networks (IJCNN), number NRC 49296. ISBN 1-4244-1380-X.

[Valdés and Gil, 1982] Valdés, J. J. and Gil, J. L. (1982). Application of geophysical and geomathematical methods in the study of the insunza karstic area (la salud, la habana). In Proceedings of the First International Colloquium of PhysicalChemistry and Karst Hydrogeology in the Caribbean Region, pages 376-384, La Habana. UNESCO.

[Valdés et al., 2007] Valdés, J. J., Orchard, R., and Barton, A. J. (2007). Exploring medical data using visual spaces with genetic programming and implicit functional mappings. In Genetic and Evolutionary Computation Conference (GECCO2007) Workshop on Medical Applications of Genetic and Evolutionary Computation (MedGEC), London, England. ISBN 978-1-59593-698-1.

[van Doren, 1991] van Doren, C. (1991). A History of Knowledge: Past, Present, and Future. Ballantine Books, New York. ISBN 0-345-37316-2.

[Wald, 1954] Wald, G. (1954). The origin of life. Scientific American.

[Wan et al., 2009] Wan, W., Mabu, S., Shimada, K., Hirasawa, K., and Hu, J. (2009). Enhancing the generalization ability of neural networks through controlling the hidden layers. Applied Soft Computing, 9(1):404 414. http://www.sciencedirect. com/science/article/B6W86-4SHF4C1-3/2/ 72403c5e1507ca3d104b56cdcbcfb104 Accessed December 2008.

[Wang et al., 1994] Wang, Z., Massimo, C. D., Tham, M. T., and Morris, A. J. (1994). A procedure for determining the topology of multilayer feedforward neural networks. Neural Networks, 7(2):291-300.

[Webb and Lowe, 1990] Webb, A. R. and Lowe, D. (1990). The optimised internal representation of multilayer classifier networks performs nonlinear discriminant analysis. Neural Networks, 3(4):367 - 375. http://www.sciencedirect.com/ science/article/B6T08-482XDCR-18/2/52ba44322181db80de728d7d48a3ff11 Accessed December 2008.

[Wells, 2006] Wells, H. G. (2006). A Short History of the World. Penguin Books Ltd, England. ISBN-13: 978-0-141-44182-5. 
[Wells, 2007] Wells, W. A. (2007). Gero Miesenbock: instructing the nervous system. Cell Biology, 177(3):374-375.

[Werbos, 1990] Werbos, P. J. (1990). Backpropagation through time: What it does and how to do it. Proceedings of the IEEE, 78(10):1550-1560.

[Whitley, 1993] Whitley, D. (1993). A genetic algorithm tutorial. http://samizdat. mines.edu/ga_tutorial Accessed December 2008.

[Whitley, 2001] Whitley, D. (2001). An overview of evolutionary algorithms: Practical issues and common pitfalls. Information and Software Technology, 43:817-831.

[Widrow and Lehr, 1990] Widrow, B. and Lehr, M. A. (1990). 30 years of adaptive neural networks: Perceptron, madaline, and backpropagation. Proceecings of the IEEE, 78(9):1415-1442.

[Wikipedia, 2008a] Wikipedia (2008a). Entry for archaeopteryx. http://en. wikipedia.org/wiki/Archaeopteryx Accessed December.

[Wikipedia, 2008b] Wikipedia (2008b). Entry for recurrent neural network. http: //en.wikipedia.org/wiki/Recurrent_neural_network Accessed December.

[Witten and Frank, 2005] Witten, I. H. and Frank, E. (2005). Data Mining: Practical machine learning tools and techniques. Morgan Kaufmann, San Francisco, second edition. http://www.cs.waikato.ac.nz/ml/weka/ Accessed December 2008.

[Wolberg and Mangasarian, 1990] Wolberg, W. H. and Mangasarian, O. L. (1990). Multisurface method of pattern separation for medical diagnosis applied to breast cytology. Proceedings of the National Academy of Sciences, 87:9193-9196. Applied Mathematics.

[Wright, 1931] Wright, S. (1931). Evolution in mendelian populations. Genetics, $16(97)$.

[Wright, 1932] Wright, S. (1932). The roles of mutation, inbreeding, crossbreeding and selection in evolution. In Proceedings of the Sixth Intermational Congress on Genetics, volume 1, pages 356-366.

[Wróblewski, 1995] Wróblewski, J. (1995). Finding minimal reducts using genetic algorithm. In Proc. of the Second Annual Joint Conference on Information Sciences.

[Wróblewski, 2001] Wróblewski, J. (2001). Ensembles of classifiers based on approximate reducts. Fundamenta Informaticae, 47.

[Yao, 1993] Yao, X. (1993). A review of evolutionary artificial neural networks. International Journal of Intelligent Systems, 8(4):539-567. 
[Yuen et al., 2009] Yuen, C., Wong, W., Qian, S., Chan, L., and Fung, E. (2009). A hybrid model using genetic algorithm and neural network for classifying garment defects. Expert Systems with Applications, 36(2, Part 1):20372047. http://www.sciencedirect.com/science/article/B6V03-4RDS40N-7/2/ f84170894639895553fd627246047ecf Accessed December 2008.

[Zhao, 2008] Zhao, Z.-Q. (2008). A novel modular neural network for imbalanced classification problems. Pattern Recognition Letters, In Press, Corrected Proof:http://www.sciencedirect.com/science/article/B6V15-4SRCJH8-2/2/ be7b0eec315b5a1ab69c0807b7bacbd0 Accessed December 2008. 


\section{Appendix A}

\section{Example NN-GEP Configuration File}

This example configuration file has been altered in such a way as to make the parameters more clear. Some non-important parameters have been deleted for space reasons. A python script (makeNNGEPparams) creates the GEP configuration file for a particular neural network architecture (nn-gep-5-1-1_ALL.params), which is then given to a batch program (generateFiles .bat b_generator.cmds), which calls a perl program (fileGenerator.pl) in order to replace the $\% @ x y z \%$ values and create all of the actual experiment configuration files, which are given to a distributed computation program (condor) in order to execute one on each compute node, which are collected with various (c) programs in order to assemble a database (db_classicalErrors.mdb), which may be analyzed.

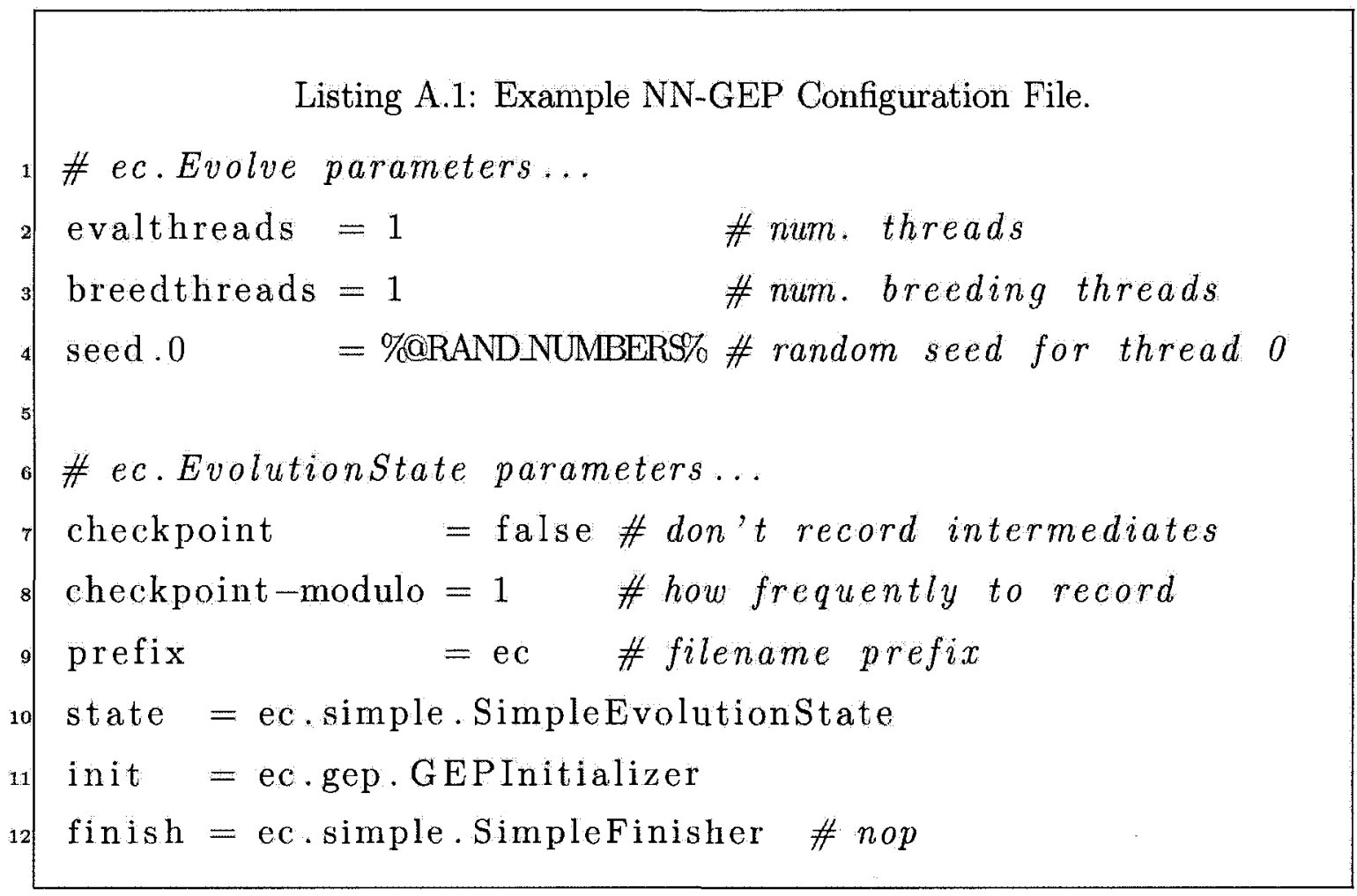




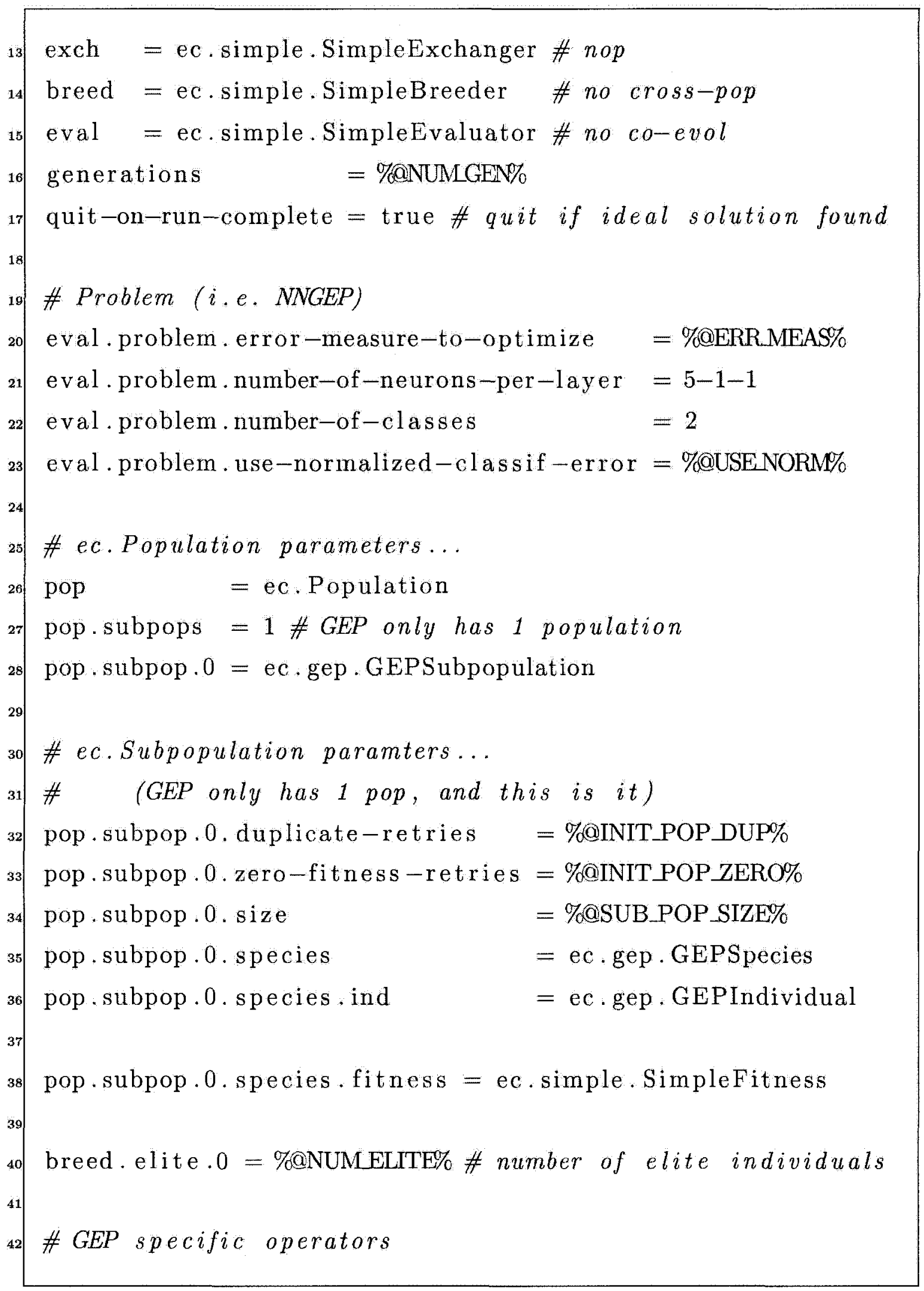




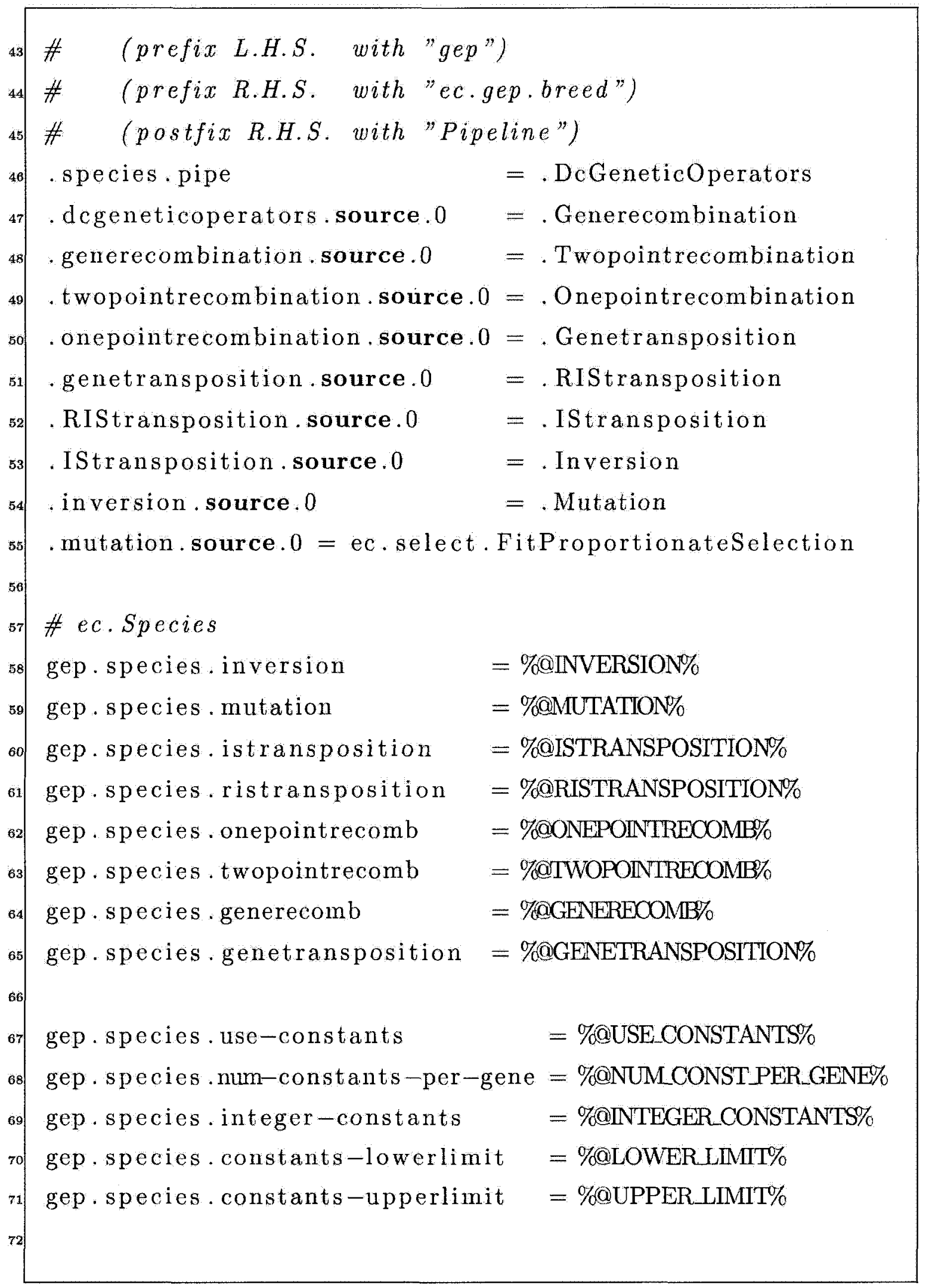




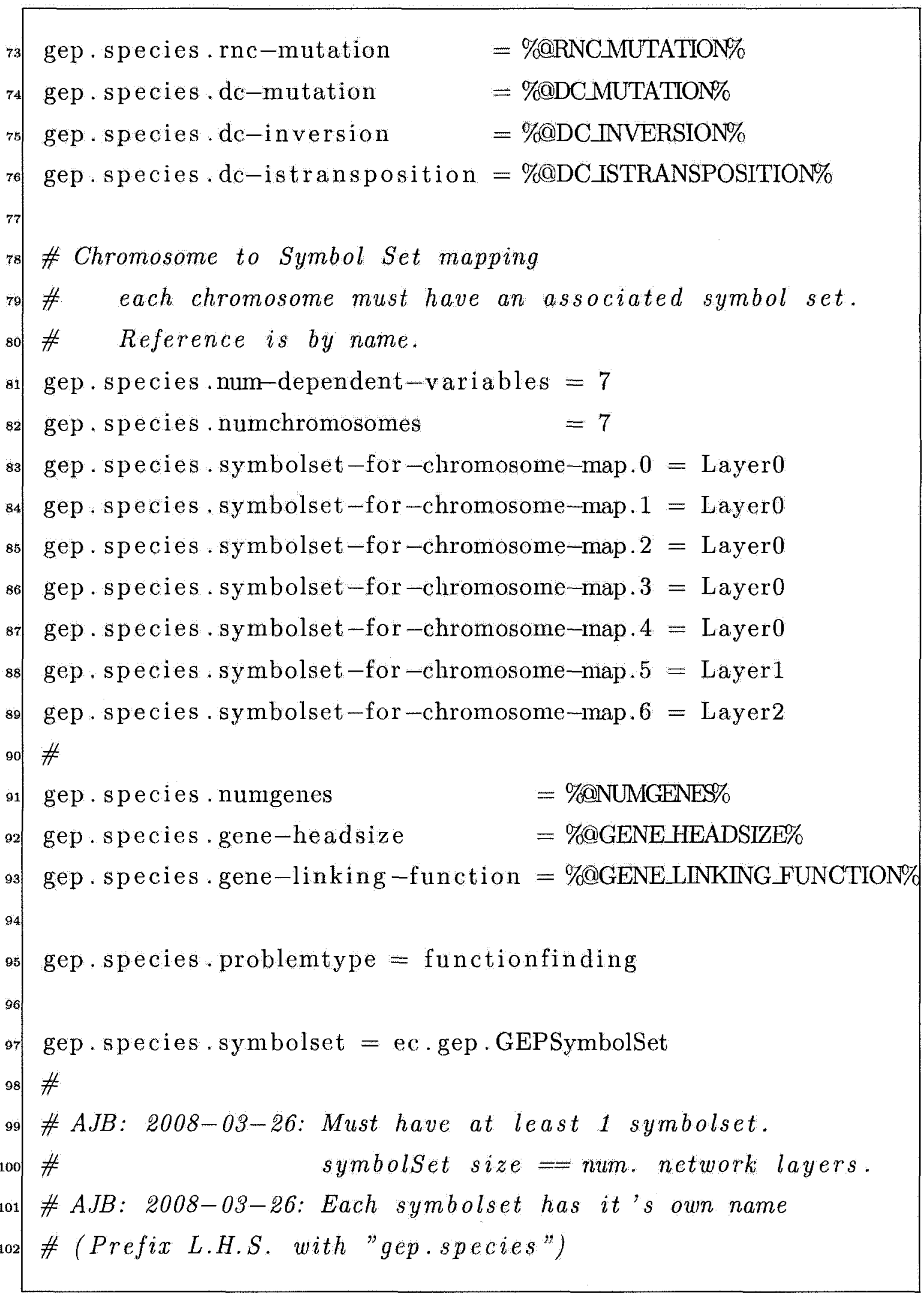




\begin{tabular}{|c|}
\hline 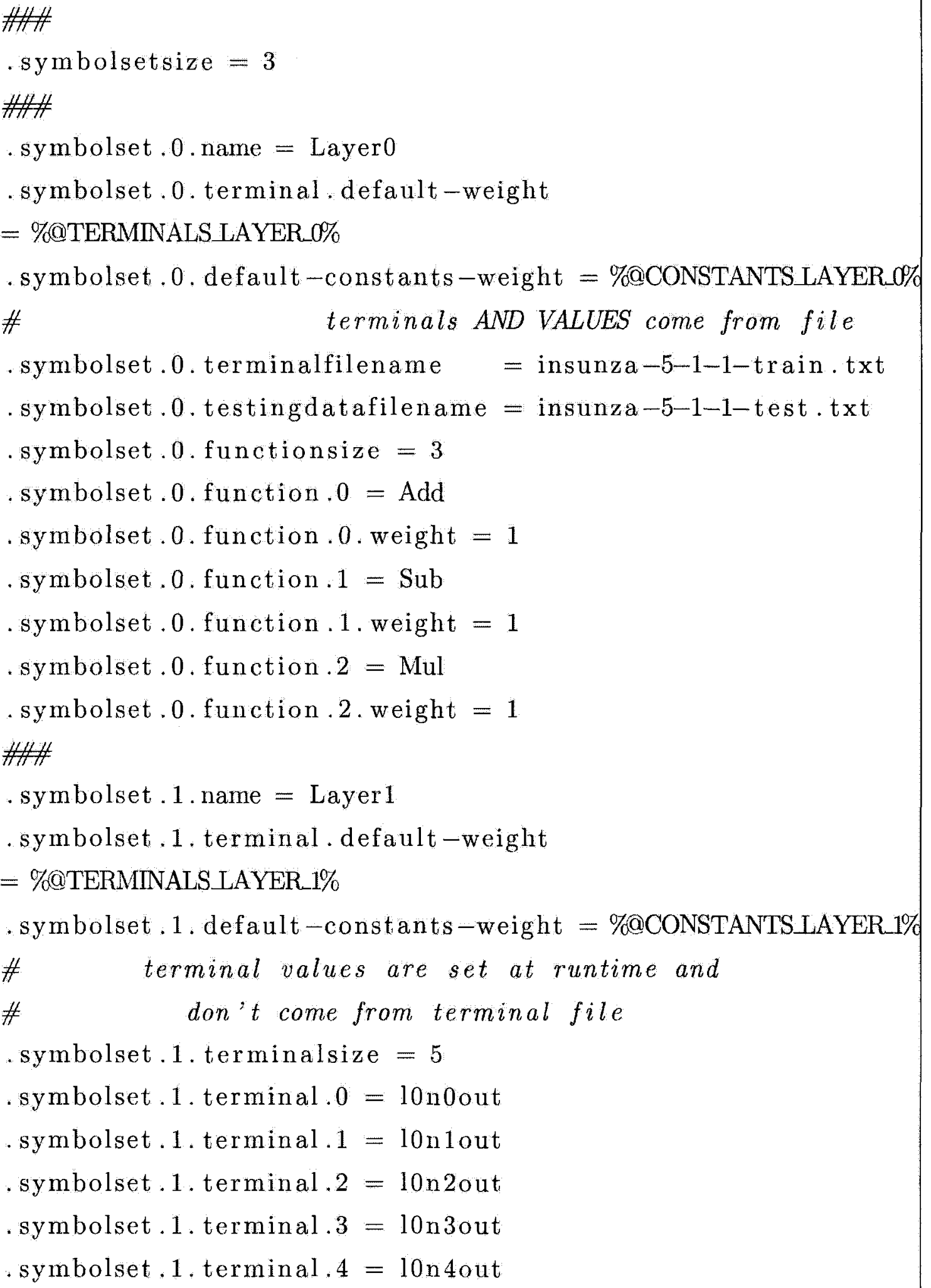 \\
\hline
\end{tabular}




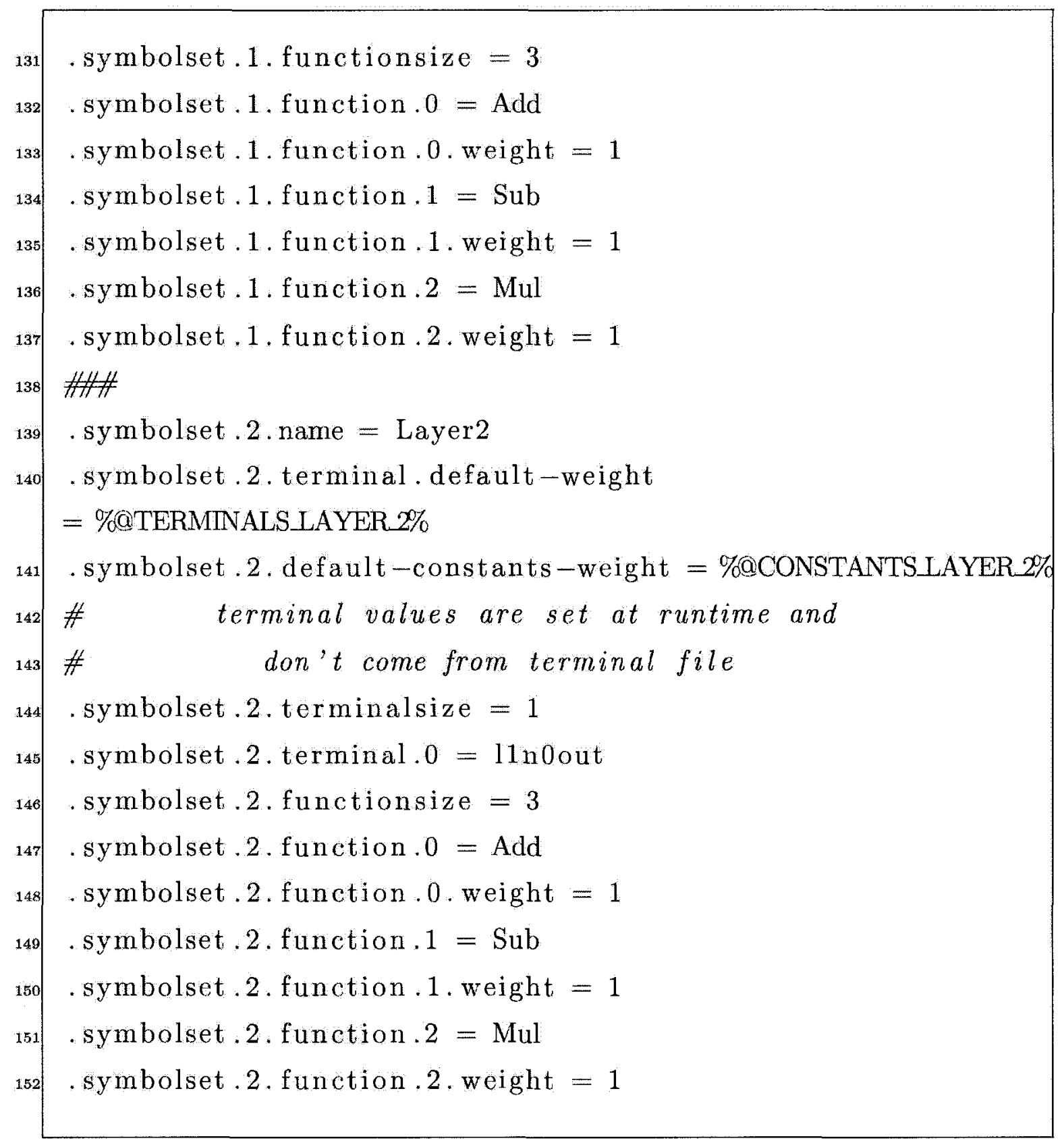




\section{Appendix B}

\section{Available Symbols within NN-GEP}

Table B.1: Available Functions within NN-GEP

\begin{tabular}{llr}
\hline Class & Input Output & Notes \\
Name & & The abstract class from which \\
\hline GEPSymbol.java & \\
& GEPTerminalSymbol, GEPFunctionSymbol and GEPConstantTer- \\
& minalSymbol are derived. They encode terminals (variables), func- \\
& tions and constants in the models produced. \\
\hline
\end{tabular}

GEPConstantTerminalSymbol.java

no values need to be set. It gets its constant value from the $\mathrm{Dc}$ part of the gene when the expression is parsed and this value is stored in a special constant expression tree node. So this Symbol is a placeholder for constants in the gene (Karva) expressions. The constant values are assigned to the constant tree nodes from the Dc portion of the gene in the order they are encountered in the Karva expression.

GEPTerminalSymbol.java

name of name of variable Supports encoding the

variable

information about a terminal: its arity (number of arguments is always $0 \ldots$ no args) and its name (symbol).

GEPFunctionSymbol.java

Supports encoding the information about a function: its arity (number of arguments) and its name (symbol). 
Table B.1 - continued from previous page

\begin{tabular}{|c|c|c|c|}
\hline $\begin{array}{l}\text { Class } \\
\text { Name }\end{array}$ & Input & Output & Notes \\
\hline Abs.java & abs & $\operatorname{abs}\left(x_{1}\right)$ & \\
\hline Acos.java & $\operatorname{acos}$ & $\operatorname{acos}\left(x_{1}\right)$ & \\
\hline Acot.java & acot & $\operatorname{acot}\left(x_{1}\right)^{1}$ & \\
\hline Acsc.java & $\operatorname{acsc}$ & $\operatorname{acsc}\left(x_{1}\right)$ & \\
\hline Add.java & + & $\left(x_{1}+x_{2}\right)$ & \\
\hline Add3.java & add3 & $\left(x_{1}+x_{2}+x_{3}\right)$ & \\
\hline Add4.java & $\operatorname{add} 4$ & $\left(x_{1}+x_{2}+x_{3}+x_{4}\right)$ & \\
\hline And.java & and & $\left(x_{1}\right.$ and $\left.x_{2}\right)$ & \\
\hline Asec.java & asec & $\operatorname{asec}\left(x_{1}\right)$ & \\
\hline Asin.java & asin & $\operatorname{asin}\left(x_{1}\right)$ & \\
\hline Atan.java & atan & $\operatorname{atan}\left(x_{1}\right)$ & \\
\hline Avg2.java & avg2 & $\left(\left(x_{1}+x_{2}\right) / 2\right)$ & \\
\hline Avg3.java & avg3 & $\left(\left(x_{1}+x_{2}+x_{3}\right) / 3\right)$ & \\
\hline Avg4.java & avg4 & $\left(\left(x_{1}+x_{2}+x_{3}+x_{4}\right) / 4\right)$ & \\
\hline Ceiling.java & ceil & $\operatorname{ceiling}\left(x_{1}\right)$ & \\
\hline Comp.java & comp & $\left(1-x_{1}\right)$ & \\
\hline Cos.java & $\cos$ & $\cos \left(x_{1}\right)$ & \\
\hline Cosh.java & $\cosh$ & $\cosh \left(x_{1}\right)$ & \\
\hline Cot.java & $\cot$ & $\cot \left(x_{1}\right)$ & \\
\hline Coth.java & coth & $\operatorname{coth}\left(x_{1}\right)$ & \\
\hline Csc.java & $\csc$ & $\csc \left(x_{1}\right)$ & Implemented as $\frac{1}{\sin (x)}$ \\
\hline Csch.java & $\operatorname{csh}^{2}$ & $\operatorname{csh}\left(x_{1}\right)$ & Implemented as $\frac{1}{\sinh (x)}$ \\
\hline Cubert.java & $3 \mathrm{RT}^{3}$ & $\left(x_{1}{ }^{-}(1.0 / 3.0)\right)$ & Math. $\operatorname{cbrt}\left(x_{1}\right)$ \\
\hline Div.java & 1 & $\left(x_{1} / x_{2}\right)$ & \\
\hline
\end{tabular}

${ }^{1} \mathrm{GEP}$ uses $\mathrm{A} \cot \left(x_{1}\right)$

${ }^{2}$ GEP uses csc, but it is an error

${ }^{3}$ GEP uses Math. $\operatorname{sqrt}\left(x_{1}\right)$, but it is an error 
Table B.1 - continued from previous page

\begin{tabular}{|c|c|c|c|}
\hline $\begin{array}{l}\text { Class } \\
\text { Name }\end{array}$ & Input & Output & Notes \\
\hline Div3.java & $\operatorname{div} 3$ & $\left(x_{1} / x_{2} / x_{3}\right)$ & \\
\hline Div4.java & $\operatorname{div} 4$ & $\left(x_{1} / x_{2} / x_{3} / x_{4}\right)$ & \\
\hline E.java & $\mathrm{e}$ & $\mathrm{e}$ & Implemented as Math.E \\
\hline Exp.java & $\exp$ & $\exp \left(x_{1}\right)$ & \\
\hline Floor.java & floor & floor $\left(x_{1}\right)$ & \\
\hline Gau.java & gau & $\exp \left(-\operatorname{pow}\left(x_{1}, 2\right)\right)$ & $e^{-\left(x_{1}\right)^{2}}$ \\
\hline Gau2.java & $\operatorname{gan} 2$ & $\exp \left(-\operatorname{pow}\left(x_{1}+x_{2}, 2\right)\right)$ & $e^{-\left(x_{1}+x_{2}\right)^{2}}$ \\
\hline Gau3.java & gau3 & $\exp \left(-\right.$ pow $\left.\left(x_{1}+x_{2}+x_{3}, 2\right)\right)$ & $e^{-\left(x_{1}+x_{2}+x_{3}\right)^{2}}$ \\
\hline Gau4.java & gau4 & $\begin{array}{l}\exp \left(-\operatorname{pow}\left(x_{1}+x_{2}+x_{3}+\right.\right. \\
\left.\left.x_{4}, 2\right)\right)\end{array}$ & $e^{-\left(x_{1}+x_{2}+x_{3}+x_{4}\right)^{2}}$ \\
\hline Inv.java & inv & $\left(1 / x_{1}\right)$ & $\frac{1}{x}$ \\
\hline Ln.java & $\ln$ & $\ln \left(x_{1}\right)$ & Math. $\log (x)$ \\
\hline Log.java & $\log$ & $\log 10\left(x_{1}\right)$ & Math. $\log 10(x)$ \\
\hline Log2.java & $\log 2$ & $\left(\ln \left(x_{1}\right) / \ln \left(x_{2}\right)\right)^{4}$ & $\begin{array}{l}\text { Math. } \log \left(x_{1}\right) / \\
\text { Math. } \log \left(x_{2}\right)\end{array}$ \\
\hline Logistic.java & $\log i$ & $\left(1 /\left(1+\operatorname{pow}\left(e,-x_{1}\right)\right)\right)$ & $\frac{1}{1+e^{-x_{1}}}$ \\
\hline Max2.java & $\max 2$ & $\max \left(x_{1}, x_{2}\right)$ & Math $\cdot \max \left(x_{1}, x_{2}\right)$ \\
\hline Max3.java & $\max 3$ & $\max 3\left(x_{1}, x_{2}, x_{3}\right)^{5}$ & \\
\hline Max4.java & $\max 4$ & $\max 4\left(x_{1}, x_{2}, x_{3}, x_{4}\right)$ & \\
\hline Min2.java & $\min 2$ & $\min \left(x_{1}, x_{2}\right)$ & \\
\hline Min3.java & $\min 3$ & $\min 3\left(x_{1}, x_{2}, x_{3}\right)$ & \\
\hline Min4.java & $\min 4$ & $\min 4\left(x_{1}, x_{2}, x_{3}, x_{4}\right)$ & \\
\hline Mod.java & $\bmod$ & $\bmod \left(x_{1}, x_{2}\right)$ & Math. IEEEremainder $\left(x_{1}, x_{2}\right)$ \\
\hline Mul.java & $*$ & $\left(x_{1}^{*} x_{2}\right)$ & \\
\hline
\end{tabular}

${ }^{4}$ GEP uses $\log 2\left(x_{1}\right)$, but that seems to be an error.

${ }^{5}$ Could be better to use max, to reduce the number of new functions. 
Table B.1 - continued from previous page

\begin{tabular}{|c|c|c|c|}
\hline $\begin{array}{l}\text { Class } \\
\text { Name }\end{array}$ & Input & Output & Notes \\
\hline Mul3.java & mul3 & $\left(x_{1}^{*} x_{2}^{*} x_{3}\right)$ & \\
\hline Mul4.java & mul4 & $\left(x_{1}{ }^{*} x_{2} * x_{3} * x_{4}\right)$ & \\
\hline Nand.java & nand & $\left(x_{1}\right.$ nand $\left.x_{2}\right)$ & \\
\hline Neg.java & neg & $\left(-x_{1}\right)$ & \\
\hline Nor.java & nor & $\left(x_{1}\right.$ nor $\left.x_{2}\right)$ & \\
\hline Not.java & not & $\left(\operatorname{not} x_{1}\right)$ & $\begin{array}{l}\left(x_{1}==0.0\right) ? 1.0: \\
0.0\end{array}$ \\
\hline Nxor.java & nxor & $\left(x_{1}\right.$ nxor $\left.x_{2}\right)$ & \\
\hline Or,java & or & $\left(x_{1}\right.$ or $\left.x_{2}\right)$ & \\
\hline Pi.java & pi & pi & Math.PI \\
\hline Pow.java & pow & $\operatorname{pow}\left(x_{1}, x_{2}\right)$ & \\
\hline Pow10.java & pow10 & pow $\left(10, x_{1}\right)$ & \\
\hline Sec.java & $\sec$ & $\sec \left(x_{1}\right)$ & 1.0/Math. $\cos \left(x_{1}\right)$ \\
\hline Sech.java & $\operatorname{sech}$ & $\operatorname{sech}\left(x_{1}\right)$ & 1.0/Math. $\cosh \left(x_{1}\right)$ \\
\hline Sin.java & $\sin$ & $\sin \left(x_{1}\right)$ & Math.sin $\left(x_{1}\right)$ \\
\hline Sinh.java & $\sinh$ & $\sinh \left(x_{1}\right)$ & Math.sinh $\left(x_{1}\right)$ \\
\hline Sqrt.java & sqrt & $\operatorname{sqrt}\left(x_{1}\right)^{6}$ & Math.sqrt $\left(x_{1}\right)$ \\
\hline Sub.java & - & $\left(x_{1}-x_{2}\right)$ & \\
\hline Sub3.java & sub3 & $\left(x_{1}-x_{2}-x_{3}\right)$ & \\
\hline Sub4.java & sub4 & $\left(x_{1}-x_{2}-x_{3}-x_{4}\right)$ & \\
\hline Tan.java & $\tan$ & $\tan \left(x_{1}\right)$ & Math $\tan \left(x_{1}\right)$ \\
\hline Tanh.java & $\tanh$ & $\tanh \left(x_{1}\right)$ & Math.tanh $\left(x_{1}\right)$ \\
\hline X2.java & $\mathrm{X} 2$ & $\left(x_{1}-2\right)$ & $x_{1} * x_{1}$ \\
\hline X3.java & $\mathrm{X} 3$ & $\left(x_{1}{ }^{\wedge}\right)$ & $x_{1} * x_{1} * x_{1}$ \\
\hline X4.java & $\mathrm{X} 4$ & $\left(x_{1}-4\right)$ & \\
\hline
\end{tabular}

${ }^{6}$ See Cubert.java for cube root 
Table B.1 - continued from previous page

\begin{tabular}{llll}
\hline Class & Input & Output & Notes \\
Name & & & \\
\hline X5.java & X5 & $\left(x_{1}{ }^{-5}\right)$ & \\
Xor.java & xor & $\left(x_{1}\right.$ xor $\left.x_{2}\right)$ & \\
\hline \hline
\end{tabular}




\section{Appendix C}

\section{Experimental Databases}

Data is stored in an Access 2003 database with retrieval details reported below.

\section{C.1 MS Access 2003 Problem}

MS Access 2003 had a serious problem. Namely, when it imports data from a *.csv file that contains commas as separators and does not have IDs the automatically generated identifiers are not sequential. For example, for one of the Insunza files, which is correct, when I imported it into an MS Access database as a table, then the generated IDs were found to not be based on row number, hence, the table becomes interleaved.

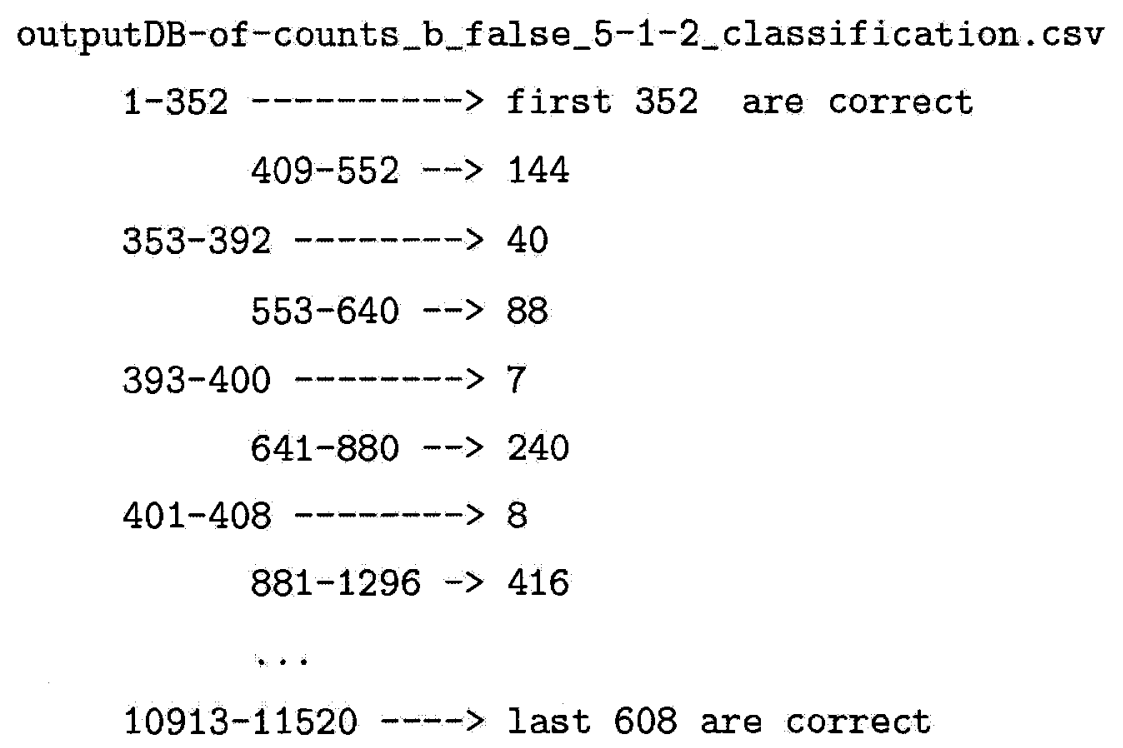

I was originally confirming the import by checking the first and last 30 to 40 rows, which are all correct, which caused me to assume the complete import was correct. To bad for me; the problem occurs in the middle. All tables were rechecked and relevant equations updated appropriately. 


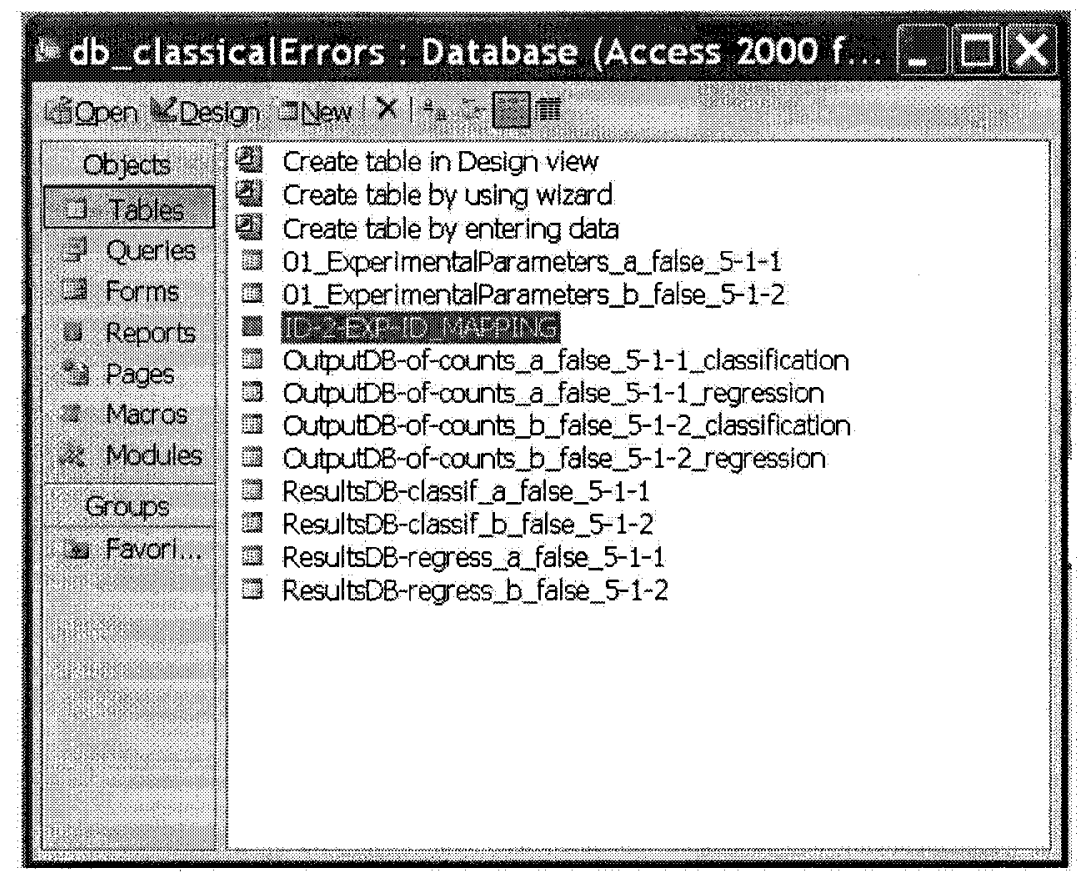

Figure C.1: Example demonstrating set of tables that may be queried to retrieve all related data for a particular neural network architecture and error measure (classical $\mathcal{E}_{C}$ or normalized $\mathcal{E}_{C_{N}}$ ). In this example, the Insunza experimental data results has one highlighted table that was constructed with a query (See Appendix C.4 on page 187).

\section{C.2 Tables in 1 of the 6 Experimental Databases}

The Insunza experimental database tables for classical error $\left(\mathcal{E}_{C}^{T r}, \mathcal{E}_{C}^{T e}\right)$ are in Fig. C.1 on page 187.

\section{C.3 Queries in 1 of the 6 Experimental Databases}

The Insunza experimental database queries for classical error $\left(\mathcal{E}_{C}^{T r}, \mathcal{E}_{C}^{T e}\right)$ are in Fig. C.2 on page 188.

\section{C.4 ID to EXP_ID Conversion Methodology}

Generation of experiments was performed via a set of tools that were created for this purpose. In particular, file system restrictions required experiments to be generated in stages. As such, this section specifies how perform the necessary join through an 


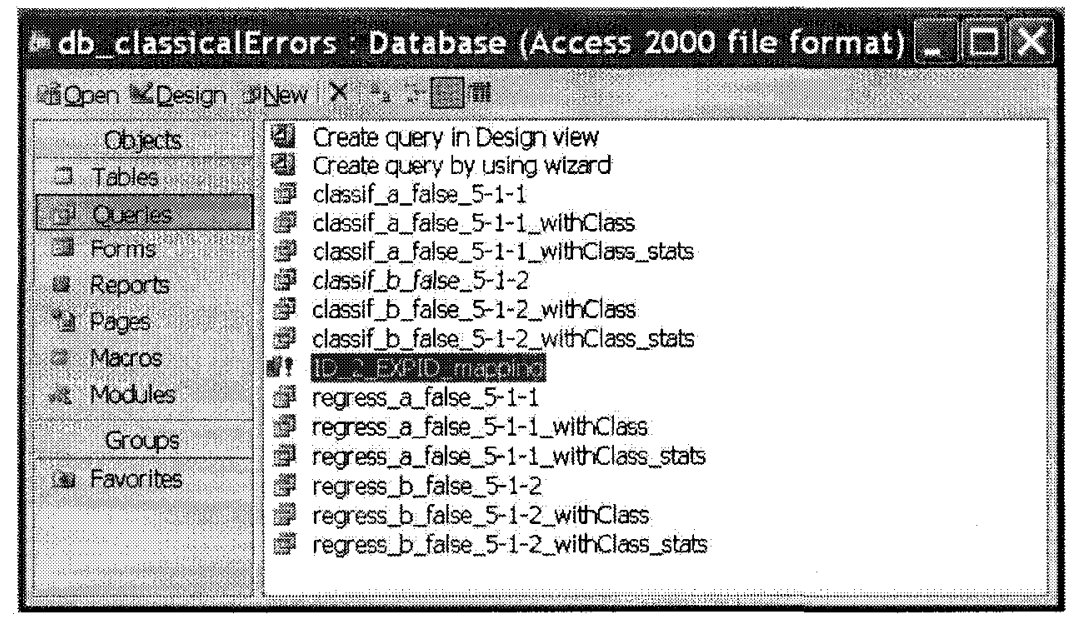

Figure C.2: Example demonstrating set of queries that have been constructed in order to retrieve all related data for a particular neural network architecture and error measure (classical $\mathcal{E}_{C}$ or normalized $\mathcal{E}_{C_{N}}$ ). In this example, the Insunza experimental data results has one highlighted queries (See Appendix C.4 on page 187).

intermediate table that needed to be constructed via a query.

1. results were generated in "classif" /"regress" pairs leading to 23, 040 experiments per group (i.e. 11,520 each). e.g. 01_ExperimentalParameters_a_true_5-1-1

$\begin{array}{ll}\operatorname{expID} & \text { OERR_TO_OPTIMIZE } \\ 1 & \text { classification } \\ 2 & \text { regression } \\ \ldots & \ldots \\ 23039 & \text { classification } \\ 23040 & \text { regression }\end{array}$

2. "classif" has 2 types; one for 1 output neuron and 1 for $>1$ output neurons (same for "regress") but they are stored in different tables

3. The ID mapping table is in Fig. C.2 on page 188 and the following query constructs it:

SELECT [ResultsDB-classif_a_false_5-1-1].ID, 


\author{
$2 *([I D]-1)+1$ AS EXP_ID_CLASSIF, \\ $2 *$ [ID] AS EXP_ID_REG \\ FROM [ResultsDB-classif_a_false_5-1-1] ;
}

\title{
C.5 Using VBA within SQL to Count Number of Constants and Variables
}

The SQL language does not support very many functions (e.g. COUNT()), however, Microsoft has extended SQL with the Visual Basic Language (VBA). Token counting is not a standard function, but the following was obtained ${ }^{1}$ :

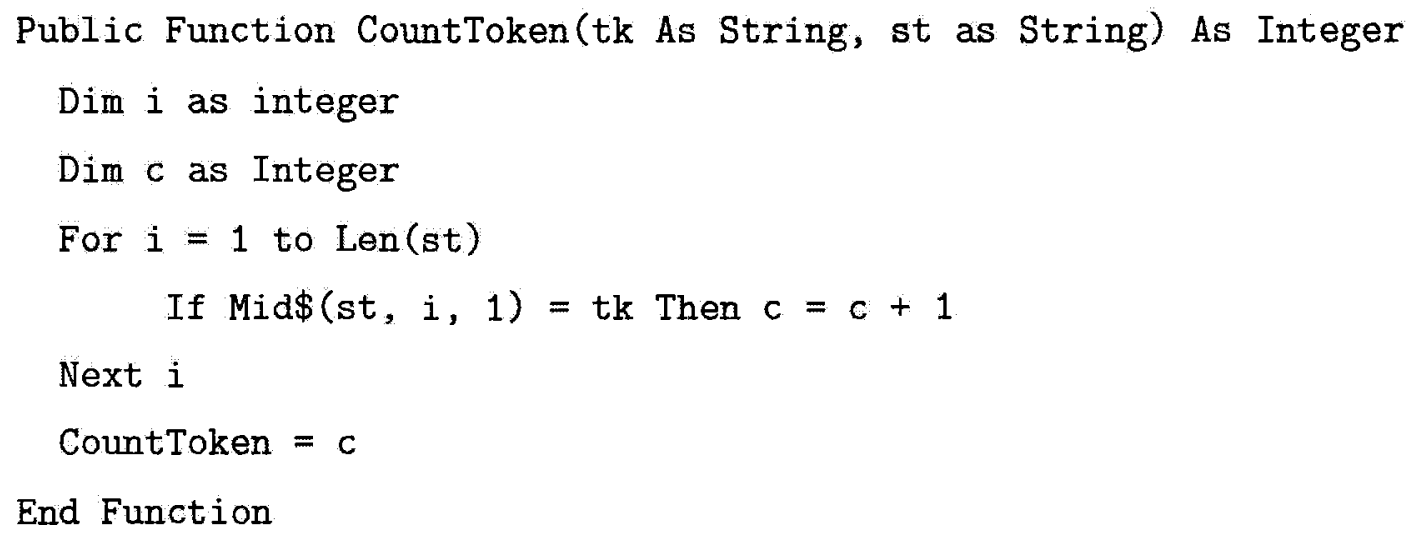

Example call: $\mathrm{a}=$ CountToken (", ", "x, a,b,c"), which will set $a$ to 3 . In order to count the number of occurrences of constants within expressions, it is noted that "." is unique. The following expressions ${ }^{2}$ may be using within the MS Access GUI:

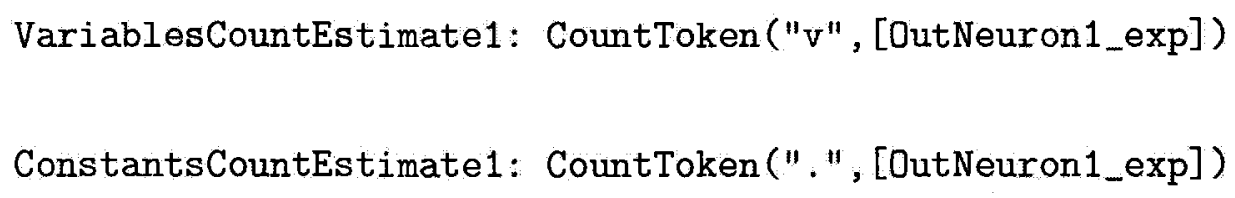

\footnotetext{
${ }^{1}$ This VBA function was obtained from: http://www.experts-exchange.com/Microsoft/ Development/MS_Access/Q_10315823.html

${ }^{2}$ These are not SQL statements. The following is an example of an SQL statement: CountToken (". ", [OutNeuron1_exp]) AS CountForNeuron1
} 


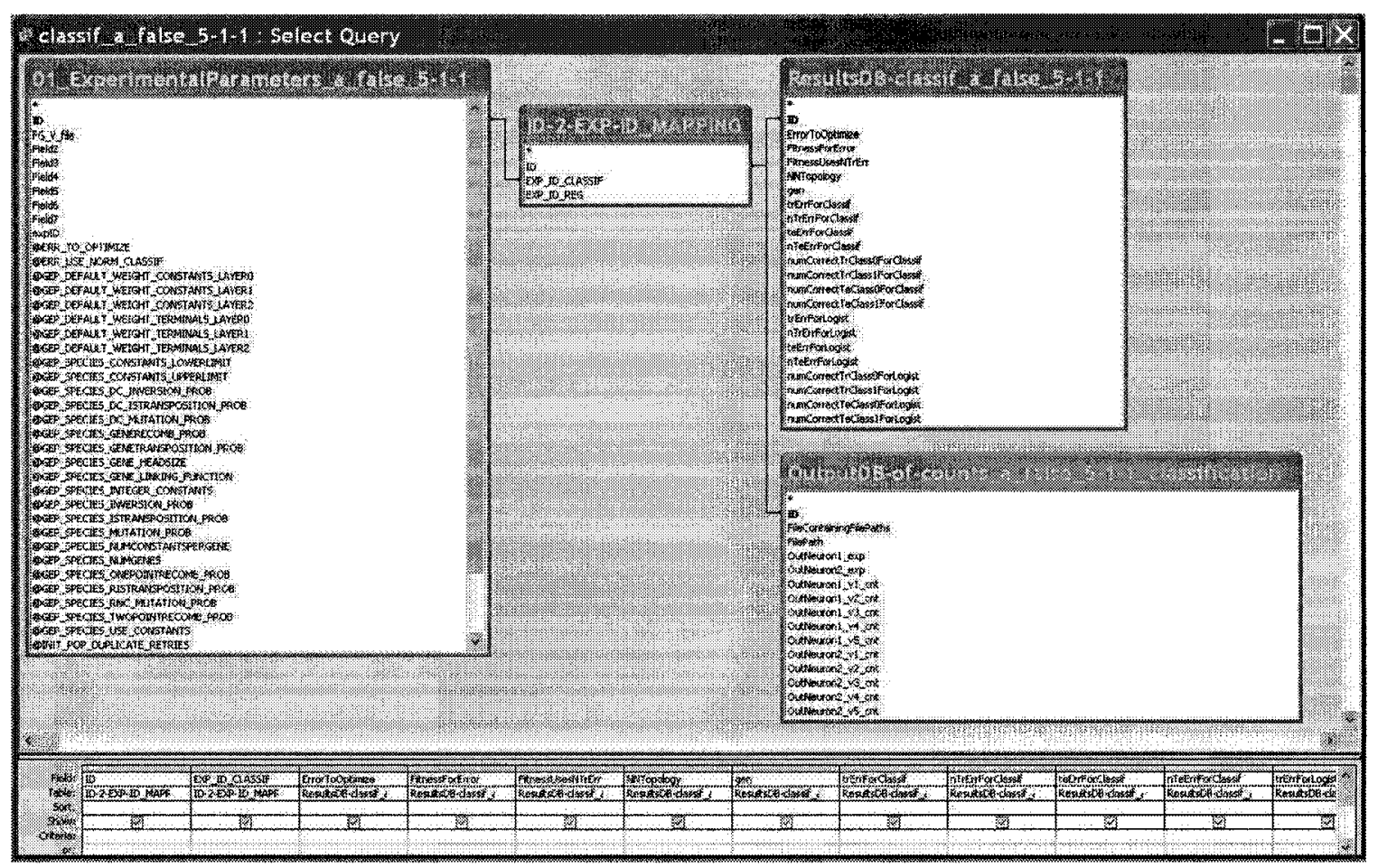

Figure C.3: Example demonstrating query to retrieve all related data (11, 520 records) for a particular neural network architecture and error measure (classical $\mathcal{E}_{C}$ or normalized $\mathcal{E}_{C_{N}}$ ). In this example, NN:5-1-1 and $\mathcal{E}_{C}^{T r}, \mathcal{E}_{C}^{T e}$ for Insunza data.

\section{C.6 Querying 1 of the 6 Databases for Related Experimental Data}

Fig. C.3 on page 190 shows how 4 tables are joined to construct a table containing all related data.

\section{C.7 Parameter Space Class Construction}

The parameter space does not have any associated classes. However, using a threshold on the associated training errors can lead to the construction of classes.

The following equations were used for Insunza $\left(\mathcal{E}_{C}\right)$ :

class: IIf ([trErrForClassif $]<0.15, \operatorname{IIf}([\operatorname{trErrForClassif}]<0.05,1,2), 3)$

class: IIf ([trErrForLogist $]<0.15, \operatorname{IIf}([\operatorname{trErrForLogist~}]<0.05,1,2), 3)$

The following equations were used for Insunza $\left(\mathcal{E}_{C_{N}}\right)$ :

class: IIf $([n \operatorname{TrErrForClassif}]<0.15, \operatorname{IIf}([n \operatorname{TrErrForClassif}]<0.072,1,2), 3)$ 
class: IIf ( [nTrErrForLogist] $<0.15, \operatorname{IIf}([n \operatorname{TrErrForLogist~}]<0.072,1,2), 3)$

The following equations were used for Breast Cancer $\left(\mathcal{E}_{C}\right)$ :

class: IIf $([\operatorname{trErrForClassif]}<0.15, \operatorname{IIf}([\operatorname{trErrForClassif}]<0.028,1,2), 3)$

class: IIf ([trErrForLogist $]<0.15, \operatorname{IIf}([\operatorname{trErrForLogist~}]<0.028,1,2), 3)$

The following equations were used for Breast Cancer $\left(\mathcal{E}_{C_{N}}\right)$ :

class: IIf ([nTrErrForClassif $]<0.15, \operatorname{IIf}([n \operatorname{TrErrForClassif]}<0.017,1,2), 3)$

class: IIf ( [nTrErrForLogist $]<0.15, \operatorname{IIf}([\mathrm{nTrErrForLogist}]<0.017,1,2), 3)$

The following equations were used for Werenskiold $\left(\mathcal{E}_{C}\right)$ :

class: IIf ([trErrForClassif $]<0.50$, IIf $([\operatorname{trErrForClassif]}<0.42,1,2), 3)$

class: IIf $([\operatorname{trErrForLogist~}]<0.50, \operatorname{IIf}([\operatorname{trErrForLogist}]<0.42,1,2), 3)$

The following equations were used for Werenskiold $\left(\mathcal{E}_{C_{N}}\right)$ :

class: IIf ([nTrErrForClassif $]<0.52, \operatorname{IIf}([n \operatorname{TrErrForClassif}]<0.489,1,2), 3)$

class: IIf([nTrErrForLogist $]<0.52, \operatorname{IIf}([n \operatorname{TrErrForLogist~}]<0.489,1,2), 3)$ 


\section{Appendix D}

\section{Further Reading}

Most important for this thesis is understanding a Neural Network, which has been examined in [Lapedes and Farber, 1988]. Second most important, is understanding genetic algorithms [Jong, 1988], [Schaffer et al., 1992], [Whitley, 1993] and genetic programming [Koza, 1995b], [Whitley, 2001], [Poli et al., 2008]. While, the third important subject is related to global optimization trends with deterministic and stochastic techniques [Pardalos et al., 2000]. Notes related to evolutionary and genetic algorithms, genetic programming, and neural-network computations from the mathematical point of view, namely, analysis of functions prescribed at permutations, are studied in [Korolev, 2007]. Finally, [Soule and Heckendorn, 2002] discusses code growth (bloat) in genetic programming, which is an important issue, but beyond the scope of this thesis. 